Emily O'Connor Maher

134 Words

\title{
A Measurement of the Charged-Current Interaction Cross Section of the Tau Neutrino
}

The Fermilab experiment E872 (DONUT) was designed to make the first observation of the tau neutrino charged-current interaction. Using a hybrid emulsion-spectrometer detector, the tau lepton was identified by its single-prong or trident decay. Six interactions were observed, of which five were in the deep inelastic scattering region. These five interaction were used to measure the charged-current cross section of the tau neutrino. To minimize uncertainties, the tau neutrino cross section was measured relative to the electron neutrino cross section. The result

$$
\frac{\sigma_{\nu_{\tau} N}^{\text {const }}}{\sigma_{\nu_{e} N}^{\text {const }}}=0.77 \pm 0.39
$$

is consistent with 1.0, which is predicted by lepton universality. The tau neutrino cross section was also measured for $115 \mathrm{GeV}$ neutrinos, which was the average energy of the interacted tau neutrinos. The result

$$
\sigma_{\nu_{\tau} N}^{\exp }=45 \pm 21 \times 10^{-38} \mathrm{~cm}^{2}
$$

is consistent with the standard model prediction calculated in this thesis,

$$
\sigma_{\nu_{\tau} N}^{\mathrm{SM}}=48 \pm 5 \times 10^{-38} \mathrm{~cm}^{2}
$$




\title{
UNIVERSITY OF MINNESOTA
}

This is to certify that I have examined this bound copy of a doctoral thesis by

\author{
Emily O'Connor Maher
}

and have found that it is complete and satisfactory in all respects and that any and all revisions required by the final examining committee have been made.

Professor Kenneth Heller

(Faculty Adviser)

GRADUATE SCHOOL 


\title{
A Measurement of the Charged-Current Interaction Cross Section of the Tau Neutrino
}

\author{
A THESIS \\ SUBMITTED TO THE FACULTY OF THE GRADUATE SCHOOL \\ OF THE UNIVERSITY OF MINNESOTA \\ BY
}

Emily O'Connor Maher

IN PARTIAL FULFILLMENT OF THE REQUIREMENTS

FOR THE DEGREE OF

DOCTOR OF PHILOSOPHY

February, 2007 


\title{
A Measurement of the Charged-Current Interaction Cross Section of the Tau Neutrino
}

\author{
by Emily O'Connor Maher \\ Under the supervision of Professor Kenneth Heller
}

\begin{abstract}
The Fermilab experiment E872 (DONUT) was designed to make the first observation of the tau neutrino charged-current interaction. Using a hybrid emulsion-spectrometer detector, the tau lepton was identified by its single-prong or trident decay. Six interactions were observed, of which five were in the deep inelastic scattering region. These five interaction were used to measure the charged-current cross section of the tau neutrino. To minimize uncertainties, the tau neutrino cross section was measured relative to the electron neutrino cross section. The result

$$
\frac{\sigma_{\nu_{\tau} N}^{\text {const }}}{\sigma_{\nu_{e} N}^{\text {const }}}=0.77 \pm 0.39
$$

is consistent with 1.0, which is predicted by lepton universality. The tau neutrino cross section was also measured for $115 \mathrm{GeV}$ neutrinos, which was the average energy of the interacted tau neutrinos. The result

$$
\sigma_{\nu_{\tau} N}^{\exp }=45 \pm 21 \times 10^{-38} \mathrm{~cm}^{2}
$$
\end{abstract}

is consistent with the standard model prediction calculated in this thesis,

$$
\sigma_{\nu_{\tau} N}^{\mathrm{SM}}=48 \pm 5 \times 10^{-38} \mathrm{~cm}^{2} .
$$




\section{Acknowledgements}

First I would like to thank my adviser, Ken Heller. Without his help, the thesis would not have been possible. I would like to thank the members of the DONUT collaboration for the help they provided during my thesis research, especially Bruce Baller, Byron Lundberg, and Vittorio Paolone who answered countless question for me.

Without my friends I never would have made it through these six years in grad school. I want to thank Ben Speakman, Jeremy Gogos, Bernie Becker, Ben Bousquet, Tau Qian, Brian Andersson, Dipu Rahman, Paul Way, Sarwa Tan, and Jorge Estevez. I am probably forgetting someone, so please forgive me. I especially want to thank the MINOS grad students who kept me sane both at the university and down in the mine and for exposing me to the night-life in Virginia. I would also like to thank all the members of the SuperOffice who helped me survive my first year.

I want to thank Lindsey Hillesheim, Melissa Elben-Zayas, and Beth Masimore for understanding. Life would have been much more difficult if I hadn't had them. I would like to thank Carolyn Erickson for being a good, although sporadic, office mate and friend.

I would like to thank my book club Soma, Urvi, Michele, Patrycia, and Tatyana for providing a reason to take a break from physics every once in awhile and talk about econ instead. I would especially like to thank Meg and Denise for their support and friendship. Life would not have been nearly as interesting without these two.

I am thankful for friends I have had along the way. I want to thank Ron, Luis, Andrew, and Adi for keeping things interesting each in their own ways.

I am grateful to my St. Paul Jazzercise class, especially Pam, Becky, Andrea, Trish, and Ellen, who kept me sane when I was in the depths of graduate school. Some weeks our Saturday morning coffees were all I could look forward to. I especially want to thank Sandy for her unwavering enthusiasm, even on crutches, and I want to especially thank Ruth for her stories that always made me smile no matter how bad my day was and our domination of the promotions; long live the JJ's! 
I would like to thank my teacher and my friend Dr. Richard Rolleigh for inspiring me to study physics. Without his encouragement and support, both during college and after, I would not be here.

Finally I would like to thank my family, my Mom, my sister Ellen, my brother Steve, and Michael, for their love and support over the years. I couldn't have done this without them. My mom was always ready to listen with unending patience; she probably knows much more about graduate school than she intended to. I want to thank my brother and my sister for keeping me grounded and making me realize that graduate school is not the most important thing in life. Although we were far apart, they were always just a phone call away. I want to thank Michael, who, aside from myself, is the most responsible for my completion. Without his continual encouragement throughout the last six years, I would not have made it. Thank you all very much. 


\section{Table of Contents}

Abstract

Acknowledgements $\quad$ ii

List of Tables $\quad$ vii

List of Figures $\quad$ ix

1 Introduction $\mathbf{1}$

1.1 Historical Background of Neutrinos . . . . . . . . . . . . . . . 1

1.2 Why Study the Tau Neutrino? . . . . . . . . . . . . . . . . . . 2

1.3 Purpose and Overview . . . . . . . . . . . . . . . 3

2 Neutrinos in the Standard Model 4

2.1 The Standard Model . . . . . . . . . . . . . . . . . . . . . . . . 4

2.2 Neutrino Interactions in the Standard Model . . . . . . . . . . . . . . 6

3 The Experimental Setup 16

3.1 Introduction . . . . . . . . . . . . . . . 16

3.2 The Neutrino Beam . . . . . . . . . . . . . . . . . . . 17

3.2 .1 The Tungsten Target . . . . . . . . . . . . . . . . . . . . . . . . . . . . 17

3.2 .2 Shielding . . . . . . . . . . . . . . . 18

3.3 The DONUT Detector . . . . . . . . . . . . . . . 20

3.3 .1 The Emulsion Target . . . . . . . . . . . . . . . 21

3.3 .2 The Spectrometer . . . . . . . . . . . . . . . . 23

4 Data Analysis and Simulation 29

4.1 The Preliminary Data Set . . . . . . . . . . . . . . . . . 29 
4.2 Spectrometer Analysis . . . . . . . . . . . . . . . . . . . . . 29

4.2.1 The Hardware Filter . . . . . . . . . . . . . . . . . . . . . . . . 30

4.2 .2 The Software Filter . . . . . . . . . . . . . . . . . . 31

4.2 .3 The Visual Filter . . . . . . . . . . . . . . . . . . . . . . 31

4.2.4 The Emulsion Volume Filter . . . . . . . . . . . . . . . . . . 32

4.3 Emulsion Data Analysis . . . . . . . . . . . . . . . . . 35

4.3 .1 Emulsion Scanning . . . . . . . . . . . . . . . . . 35

4.3.2 Location in Emulsion Data . . . . . . . . . . . . . . . . . 38

4.3.3 Additional Emulsion Information . . . . . . . . . . . . . . . . 39

4.4 Event Simulation . . . . . . . . . . . . . . . . . . . . . . 44

4.5 Parameters for Statistical Analysis . . . . . . . . . . . . . . . . 47

4.6 Event Classification . . . . . . . . . . . . . . . . . . . 57

4.6.1 Muon Neutrino Charged-Current Interactions . . . . . . . . . . . . 58

4.6.2 Electron Neutrino Charged-Current Interactions _ . . . . . . . . . 58

4.6.3 Tau Neutrino Charged-Current Interactions . . . . . . . . . . . . . . 60

4.6.4 Charm Particles . . . . . . . . . . . . . . . . . . . 61

4.6.5 Neutral-Current Interaction . . . . . . . . . . . . . . . . 61

4.7 Number of Events . . . . . . . . . . . . . . . . . . . . 61

5 Tau and Charged Charm Candidates $\quad 63$

5.1 Tau Candidates . . . . . . . . . . . . . . . . . . . . . 64

5.2 Charm Candidates . . . . . . . . . . . . . . . . . . 105

5.3 Statistical Analysis of Tau and Charm Candidates . . . . . . . . . . . . 129

5.3 .1 Prior Probability . . . . . . . . . . . . . . . . 129

5.3 .2 Probability Density . . . . . . . . . . . . . . . 136

5.3 .3 Results . . . . . . . . . . . . . . . . . . 147

5.3.4 Testing the Statistical Analysis . . . . . . . . . . . . . 147

6 Cross Section Analysis $\quad 149$

6.1 Cross Section Formula . . . . . . . . . . . . . . . . . . . 150

6.2 Quantities for Cross Section Calculation . . . . . . . . . . . . . 151

6.2.1 The Number of Observed Tau Neutrino Interaction . . . . . . . . . . 151

6.2 .2 Efficiencies . . . . . . . . . . . . . . . . . . 151

6.2.3 Charged-Current Neutrino Cross Section . . . . . . . . . . . . . . . . 154

6.2.4 The Kinematic Factor . . . . . . . . . . . . . . . . . . . . . . . . . . 154 
6.2.5 Neutrinos Traversing the Target . . . . . . . . . . . . . . . . 155

6.2.6 Produced Energy Spectra . . . . . . . . . . . . . . . . . . 159

6.2.7 The Number of Scattering Centers . . . . . . . . . . . . . . . . 160

6.2.8 The Number of Protons on Target . . . . . . . . . . . . . . . . 161

6.2 .9 Acceptance . . . . . . . . . . . . . . . . 161

6.3 Uncertainties . . . . . . . . . . . . . . . . . . . . . . . . . 162

6.3 .1 The Statistical Uncertainties . . . . . . . . . . . . . . . . 162

6.3.2 The Systematic Uncertainties . . . . . . . . . . . . . . . . . . 162

6.4 Results . . . . . . . . . . . . . . . . . . . . 163

6.4.1 Relative Measurement of Tau to Electron . . . . . . . . . . . . . 163

6.4.2 Relative Measurement of Tau to Muon . . . . . . . . . . . . . . . . . 164

6.4.3 Relative Measurement of Electron to Muon . . . . . . . . . . . . . . 164

6.4.4 Absolute Measurement of the Cross Sections . . . . . . . . . . . . 165

6.4.5 Consistency with Theoretical Data . . . . . . . . . . . . . 166

$\begin{array}{llr}7 \text { Conclusion } & 170\end{array}$

Appendix A. The Deep Inelastic Scattering Region $\quad 174$

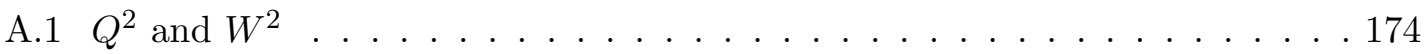

A.2 Estimating the Energy of the Tau Neutrino . . . . . . . . . . . . . . 175

A.3 Estimating the Energy of the Tau Lepton . . . . . . . . . . . 176

A.4 Data . . . . . . . . . . . . . . . . . . . . 178

A.5 Results . . . . . . . . . . . . . . . . . . 178

A.6 Conclusion . . . . . . . . . . . . . . . . . . . . 178

$\begin{array}{lr}\text { References } & 180\end{array}$ 


\section{List of Tables}

3.1 The composition of the emulsion modules . . . . . . . . . . . . . 22

4.1 Characteristics of the four run periods . . . . . . . . . . . 30

4.2 Experimental measurements for $b$ and $n$ for $800 \mathrm{GeV}$ protons . . . . . . 45

4.3 Composition of the data set . . . . . . . . . . . . . . . . . 62

5.1 Parameters for tau candidate $3024 \_30175 \ldots \ldots$. . . . . . . . . 64

5.2 Track information for tau candidate $3042 \_30175$. . . . . . . . . . . . . . . 64

5.3 Parameters for tau candidate 3039_01910 . . . . . . . . . . . . . . . 69

5.4 Track information for tau candidate 3039_01910 . . . . . . . . . . . . . 69

5.5 Parameters for tau candidate $3263 \_25102 \ldots \ldots$. . . . . . . . 75

5.6 Track information for tau candidate $3263 \_25102 \ldots \ldots$. . . . . . . . 75

5.7 Parameters for tau candidate $3333 \_17665 \ldots \ldots \ldots \ldots$. . . . . . . 80

5.8 Track information for tau candidate $3333 \_17665 \ldots \ldots$. . . . . . . 80

5.9 Parameters for tau candidate $3140 \_22143 \ldots \ldots$. . . . . . . . . . . 85

5.10 Track information for tau candidate $3140 \_22143 \ldots$. . . . . . . . . . . 86

5.11 Parameters for tau candidate $3296 \_18816 \ldots \ldots$. . . . . . . . . . . 91

5.12 Track information for tau candidate $3296 \_18816 \ldots \ldots$. . . . . . . 91

5.13 Parameters for tau candidate $3334 \_19920$. . . . . . . . . . . . . . . . . . 99

5.14 Track information for tau candidate 3334_19920 . . . . . . . . . . . . . 100

5.15 Parameters for charm candidate 2846_09042 . . . . . . . . . . . . . . 105

5.16 Track information for charm candidate 2846_09042 . . . . . . . . . . . . . 105

5.17 Parameters for charm candidate $3065 \_03238$. . . . . . . . . . . . . . 110

5.18 Track information for charm candidate $3065 \_03238$. . . . . . . . . . . . 110

5.19 Parameters for charm candidate $3227 \_03420$. . . . . . . . . . . . . . . . 119

5.20 Track information for charm candidate $3227 \_03420$. . . . . . . . . . . . . 119

5.21 Parameters for charm candidate $3245 \_22786$. . . . . . . . . . . . . . . . 124

5.22 Track information for charm candidate $3245 \_22786$. . . . . . . . . . . . . 124 
5.23 The probability of a charm quark producing a $D^{ \pm}, D^{0}$, and $\Lambda_{c} \ldots \ldots . \ldots 132$

5.24 Prior probability for background charm kink events . . . . . . . . . . . 132

5.25 Prior probability for background charm trident events . . . . . . . . . . 133

5.26 Prior probabilities for background kink interaction events . . . . . . . . . 134

5.27 Prior probabilities for background trident interaction events . . . . . . . . . 134

5.28 Prior probabilities for background trident interaction events with two identified muons . . . . . . . . . . . . . . . . . . . . . 135

5.29 Prior probabilities for the all tau and charged charm candidates . . . . . . 136

5.30 The five parameters used in the kink analysis . . . . . . . . . . 137

5.31 The four parameters used in the trident analysis . . . . . . . . . . 137

5.32 Probability density for kink and trident candidates . . . . . . . . . . . . 138

5.33 The relative probabilities for all tau and charm candidates . . . . . . . . 147

5.34 The results of a test of the statistical analysis . . . . . . . . . . . . . 148

6.1 Efficiencies of selection criteria required to identify the tau neutrino kink interaction . . . . . . . . . . . . . . . . . . 153

6.2 Efficiencies of selection criteria required to identify the tau neutrino trident interaction . . . . . . . . . . . . . . . . . . . 153

6.3 Summary of all efficiencies for electron, muon, tau kink, and tau trident charged-current interactions . . . . . . . . . . . . . . . . . . . 154

6.4 Summary of $\alpha$ for the charm production cross section . . . . . . . . 157

6.5 Charm production cross sections for $800 \mathrm{GeV}$ protons . . . . . . . . . 158

6.6 Charm and tau lepton branching ratios . . . . . . . . . . . . . . 158

6.7 The number of scattering centers per $\mathrm{cm}^{2}$ in each module for each period . 161

6.8 The number of protons on target for each period . . . . . . . . . . . . . 162

A.1 Parameters used in the calculation of $Q^{2}$ and $W^{2} \ldots \ldots \ldots$. . . . . . . . . . . . . . . .

A.2 $W^{2}$ and $Q^{2}$ for all tau candidates . . . . . . . . . . . . 179 


\section{List of Figures}

2.1 The basic interactions of the charged-current weak interaction . . . . . . . 5

2.2 The basic interactions of the neutral-current weak interaction . . . . . . . 5

2.3 The Feynman diagram for a tau neutrino-nucleon interaction $\ldots$. . . . . . 7

2.4 The parton distribution functions for the $u$ and $d$ quarks for $E_{\nu}=115 \mathrm{GeV} 11$

2.5 The theoretical tau neutrino and anti-neutrino charged-current cross sections divided by energy as a function of energy . . . . . . . . . . . . . . 13

2.6 The theoretical muon neutrino and anti-neutrino charged-current cross sections divided by energy as a function of energy . . . . . . . . . . . . . . . 14

2.7 The experimental muon neutrino and anti-neutrino charged-current cross sections divided by energy as a function of energy . . . . . . . . . . . . 15

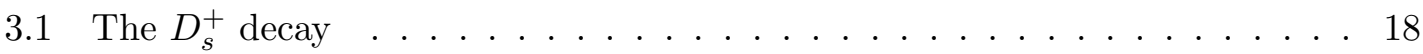

3.2 Overview of the shielding f . . . . . . . . . . . . . . 19

3.3 The emulsion detector . . . . . . . . . . . . . . 20

3.4 Emulsion sheet configurations . . . . . . . . . . . . . . . . . . 22

3.5 Overview of the DONUT spectrometer . . . . . . . . . . . . 23

3.6 Scintillating fiber tracker stations . . . . . . . . . . . . . . . . 24

3.7 Four stages of amplification of the scintillating fiber output . . . . . . . 25

3.8 The electromagnetic calorimeter f . . . . . . . . . . . . . 27

4.1 The X-view of the spectrometer data for event 3039_00914 . . . . . . . . . . 33

4.2 The U-view of the spectrometer data for event 3039_00914 . . . . . . . . . . 34

4.3 The X-view of the spectrometer data for event 2852_04994 . . . . . . . . . . 36

4.4 The U-view of the spectrometer data for event 2852_04994 . . . . . . . . . . 37

4.5 The emulsion data for a neutrino interaction . . . . . . . . . . . . . 40

4.6 The emulsion data for the same neutrino event including all background data 41

4.7 Comparison of the spectrometer and emulsion momentum measurements . . 43

4.8 The kink event parameters . . . . . . . . . . . . . . . . . . . . . 48 
4.9 The polar angle,$\Delta \phi \ldots \ldots \ldots \ldots \ldots$

4.10 The trident parameters . . . . . . . . . . . . . . 50

4.11 Distributions of $\theta$ for simulated tau, charm, and interaction events . . . . 51

4.12 Distributions of $\Delta \Phi$ for simulated tau, charm, and interaction events . . . . 53

4.13 Distributions of $L$ for simulated tau, charm, and interaction events . . . . . 54

4.14 Distributions of $\alpha$ for simulated tau, charm, and interaction events . . . . . 55

4.15 Distributions of $P_{\mathrm{d}}$ for simulated tau, charm, and interaction events . . . . 56

4.16 Distributions of $\Sigma I P$ for simulated tau, charm, and interaction events . . . 57

4.17 Example of an electron neutrino charged-current interaction . . . . . . . . 59

5.1 The X-view in the spectrometer for tau candidate $3024 \_30175$. . . . . . . . 65

5.2 The U-view in the spectrometer for tau candidate 3024_30175 . . . . . . . . 66

5.3 The V-view in the spectrometer for tau candidate 3024_30175 . . . . . . . . 67

5.4 The emulsion data for tau candidate $3024 \_30175$. . . . . . . . . . . . 68

5.5 The X-view in the spectrometer for tau candidate 3039_01910 . . . . . . . . 70

5.6 The U-view in the spectrometer for tau candidate 3039_01910 . . . . . . . . 71

5.7 The V-view in the spectrometer for tau candidate 3039_01910 . . . . . . . . 72

5.8 The emulsion data for tau candidate 3039_01910 . . . . . . . . . . . . . 73

5.9 A close-up of the vertex in the emulsion data . . . . . . . . . . . . . . 74

5.10 The X-view in the spectrometer for tau candidate 3263_25102 . . . . . . 76

5.11 The U-view in the spectrometer for tau candidate 3263_25102 . . . . . . . . 77

5.12 The V-view in the spectrometer for tau candidate 3263_25102 . . . . . . . . 78

5.13 The emulsion data for tau candidate $3263 \_25102 \ldots$. . . . . . . . . 79

5.14 The X-view in the spectrometer of tau candidate $3333 \_17665 \ldots$. . . . . 81

5.15 The U-view in the spectrometer of tau candidate 3333_17665 . . . . . . . . 82

5.16 The V-view in the spectrometer for tau candidate 3333_17665 . . . . . . . 83

5.17 The emulsion data for tau candidate 3333_17665 . . . . . . . . . . . 84

5.18 The X-view in the spectrometer for tau candidate $3140 \_22143$. . . . . . . . 87

5.19 The U-view in the spectrometer for tau candidate $3140 \_22143$. . . . . . . . 88

5.20 The V-view in the spectrometer for tau candidate $3140 \_22143$. . . . . . . . 89

5.21 The emulsion data for tau candidate $3140 \_22143$. . . . . . . . . . . . . . . 90

5.22 The X-view in the spectrometer for tau candidate 3296_18816 with the projections of the tracks from the primary vertex . . . . . . . . . . 92

5.23 The U-view in the spectrometer for tau candidate 3296_18816 with the projections of the tracks from the primary vertex $\ldots \ldots \ldots 93$ 
5.24 The V-view in the spectrometer for tau candidate 3296_18816 with the projections of the tracks from the the primary vertex . . . . . . . . . . 94

5.25 The X-view in the spectrometer for tau candidate 3296_18816 with the projections of the tracks from the secondary vertex . . . . . . . . . 95

5.26 The U-view in the spectrometer for tau candidate 3296_18816 with the projections of the tracks from the secondary vertex . . . . . . . . . . 96

5.27 The V-view in the spectrometer for tau candidate 3296_18816 with the projections of tracks from the secondary vertex . . . . . . . . . . . . . . 97

5.28 The emulsion data for tau candidate $3296 \_18816 \ldots$. . . . . . . . . . . . 98

5.29 The X-view in the spectrometer data for tau candidate 3334_19920 . . . . . 101

5.30 The U-view in the spectrometer data for tau candidate 3334_19920 . . . . . 102

5.31 The V-view in the spectrometer data for tau candidate 3334_19920 . . . . . 103

5.32 The emulsion data for tau candidate $3334 \_19920$. . . . . . . . . . . . . . . 104

5.33 The X-view in the spectrometer for charm candidate 2846_09042 . . . . . . 106

5.34 The U-view in the spectrometer for charm candidate 2846_09042 . . . . . . 107

5.35 The V-view in the spectrometer for charm candidate 2846_09042 . . . . . . 108

5.36 The emulsion data for charm candidate 2846_09042 . . . . . . . . . . . . . . 109

5.37 The X-view in the spectrometer data for charm candidate 3065_03238 with projections of the tracks from the primary vertex . . . . . . . . . . . . . 112

5.38 The U-view in the spectrometer data for charm candidate 3065_03238 with projections of the tracks from the primary vertex . . . . . . . . . 113

5.39 The V-view in the spectrometer data for charm candidate 3065_03238 with projections of the tracks from the primary vertex . . . . . . . . . . 114

5.40 The X-view in the spectrometer data for charm candidate 3065_03238 with projections of the tracks from the secondary vertex . . . . . . . . . . . 115

5.41 The U-view in the spectrometer data for charm candidate 3065_03238 with projections of the tracks from the secondary vertex . . . . . . . . . 116

5.42 The V-view in the spectrometer data for charm candidate 3065_03238 with projections of the tracks from the secondary vertex . . . . . . . . . . . 117

5.43 The emulsion data for charm candidate 3065_03238 . . . . . . . . . . . . 118

5.44 The X-view in the spectrometer data for charm candidate 3227_03420 . . . 120

5.45 The U-view in the spectrometer data for charm candidate 3227_03420 . . 121

5.46 The $\mathrm{V}$-view in the spectrometer data for charm candidate 3227_03420 . . 122

5.47 The emulsion data for charm candidate $3227 \_03420$. . . . . . . . . . . . 123

5.48 The X-view of the spectrometer data for charm candidate 3245_22786 . . 125 
5.49 The U-view of the spectrometer data for charm candidate 3245_22786 . 126

5.50 The V-view of the spectrometer data for charm candidate 3245_22786 . . 127

5.51 The emulsion data for charm candidate $3245 \_22786$. . . . . . . . . . . . . 128

5.52 Charm production in neutrino-nucleon interactions . . . . . . . . . . . 131

5.53 Distributions of $\alpha$ with event data . . . . . . . . . . . . . . 139

5.54 Distributions of $P_{\mathrm{d}}$ with event data . . . . . . . . . . . . . . . . 140

5.55 Distributions of $P_{t}$ for simulated tau events . . . . . . . . . . . . . 141

5.56 Distributions of $p_{t}$ for simulated interaction events . . . . . . . . . . . . 142

5.57 Distributions of $\theta$ with event data . . . . . . . . . . . . . 143

5.58 Distributions of $\Delta \Phi$ with event data . . . . . . . . . . . . . . 144

5.59 Distributions of $L$ with event data . . . . . . . . . . . . . . . . 145

5.60 Distributions of $\Sigma I P$ with event data . . . . . . . . . . . . . . 146

6.1 Kinematic factor for the tau neutrino and anti-neutrino . . . . . . . . . . 155

6.2 The energy spectrum of produced tau neutrinos . . . . . . . . . . . . . . 159

6.3 The energy spectrum of produced electron neutrinos . . . . . . . . . . 160

6.4 Predicted and measured cross section ratios . . . . . . . . . . . . . . 167

6.5 Predicted and measured tau neutrino cross sections evaluated at $115 \mathrm{GeV}$. 169

A.1 The angles used in the calculation of the tau neutrino energy . . . . . . . . 175

A.2 A comparison of the daughter's momentum and the momentum of the tau lepton for the kink events . . . . . . . . . . . . . . 176

A.3 A comparison of the sum of the three daughters's momenta and the momentum of the tau lepton for the trident events . . . . . . . . . . . . 177 


\section{Chapter 1}

\section{Introduction}

The standard model of particle physics is currently the most widely accepted description of the physical world. The DONUT experiment at Fermi National Accelerator Laboratory was designed to test the standard model by making the first observation of the interaction of the tau neutrino and making the first measurement of the cross section of the tau neutrino. This thesis describes the experimental design and analysis techniques used to identify tau neutrino interactions and the measurement of the tau neutrino cross section using data from the DONUT experiment.

\subsection{Historical Background of Neutrinos}

In 1929 Wolfgang Pauli was the first to propose the existence of the neutrino as "a desperate remedy to save the principle of energy conservation in beta decay..." [1]. At the time, beta decay was understood to be a two-body decay of a neutron to a proton and an electron. According to the conservation of energy, the electron should have a single-valued energy. However, experiments measured a spectrum of energies for these electrons. Pauli explained this discrepancy by introducing the neutrino, a small, massless particle. If a neutrino is also produced in beta decay, the decay is no longer a two-body decay, and the energy of the electron should vary.

Almost 30 years later in 1956 Cowan and Reins directly observed the first neutrino interaction [2]. At the Savannah River nuclear reactor in South Carolina, they constructed a large tank of water. In the tank, they detected the distinct signature of the nuclear interaction: $\overline{\nu_{e}}+p \rightarrow n+e^{+}$, which is initiated by the electron anti-neutrino.

The second type of neutrino was observed by Lederman, Schwartz, and Steinberger in 
1962 [3] using a beam of protons. The interaction of the protons in the target material created pions, which decayed into muons and neutrinos. Using magnets, the muons were removed from the beam. The neutrinos continued to a detector. If there were a distinct muon neutrino, this neutrino would create muons rather than electrons when it interacted. Muons, not electrons, were observed in the detector, providing evidence for the muon neutrino.

In 2001 the DONUT experiment made the first observation of the tau neutrino interaction [6]. The DONUT experiment used $800 \mathrm{GeV}$ protons from the Tevatron at Fermi National Accelerator Laboratory; the protons interacted in a tungsten target and produced a beam of neutrinos. The neutrinos traversed a hybrid spectrometer-emulsion detector, where a small fraction interacted. These interactions were observed in the detector and classified as muon, electron, or tau neutrino interactions; six tau neutrino interactions were observed.

\subsection{Why Study the Tau Neutrino?}

Neutrinos, unlike other particles, only interact through one type of interaction, the weak interaction. As a result, neutrino interactions are simpler than the other fundamental particle interactions; thus, neutrinos are a good tool to probe the standard model. The first observations of the tau neutrino provided the first opportunity to measure the cross section of the tau neutrino. Since the standard model predicts the tau neutrino cross section, this measurement provided a test of the standard model.

In addition to testing the standard model, the cross section will aid in the study of neutrino oscillations. Neutrino oscillations provided the first definitive evidence of physics beyond the standard model. In 1998, the first experimental evidence for neutrino oscillations was obtained using atmospheric neutrinos [7]; in 2001, solar neutrinos were used to verify this evidence [8]. Currently experiments are being designed to study neutrino oscillations in more detail. One such experiment, the OPERA experiment, will search for tau neutrino appearance in a muon neutrino beam [9]; the tau neutrino cross section will aid in explaining the OPERA results.

Neutrino astronomy is a relatively new field which uses neutrinos to study astronomical objects, such as the sun, supernova, and gamma ray bursts. The first instance of neutrino astronomy was the measurement of the neutrino flux from the sun to test solar models [10]. In addition, neutrinos may play a part in the dark matter problem [11] and, according to the big bang theory, some may be remnants of the creation of the universe [12]. Studying 
these neutrinos will lead to a greater understanding of the universe and its origin.

\subsection{Purpose and Overview}

This thesis describes the measurements of the tau neutrino cross section using techniques which determined the relative and absolute cross sections. In the relative measurement, certain systematic errors canceled. The absolute measurement provided a check of the relative measurements. To test the validity of the technique, the electron and muon neutrino cross sections were measured and compared to their known values. The charm decays, which were potential backgrounds to the tau neutrino interactions, were used to check the selection procedure, which selected the tau neutrino candidates.

The rest of the thesis progresses as follows: Ch. 2 provides an overview of neutrinos in the standard model and the standard model prediction for the tau neutrino cross section. Ch. 3 describes the experimental design: the neutrino beam, the detector, and the rationale behind the design. Ch. 4 provides a summary of the data acquisition and reduction and the method of classifying events by type. In Ch. 5 the tau neutrino and charged charm candidates are each discussed in detail. A statistical analysis performed on both types of candidates is explained and the results presented in Ch. 5. Ch. 6 includes the details of the cross section measurements and their comparisons with the predicted values. Ch. 7 briefly summarizes the conclusions obtained in this thesis and the implications of these conclusions. Appendix A presents the calculation of the deep inelastic scattering variables, which are explained in Ch. 2, for each tau neutrino interaction candidate. 


\section{Chapter 2}

\section{Neutrinos in the Standard Model}

\subsection{The Standard Model}

According to the standard model, all matter consists of three types of fundamental particles: quarks, leptons, and gauge bosons. Quarks come in six flavors: up, down, charm, strange, top, and bottom. Leptons come in six flavors: electron, electron neutrino, muon, muon neutrino, tau, and tau neutrino. Four types of gauge bosons mediate the fundamental interactions between the quarks and leptons. The gluon mediates the strong interaction, which occurs between quarks. The photon mediates the electromagnetic interaction, which occurs between all particle with electric charge. The $W^{ \pm}$and $Z^{0}$ gauge bosons both mediate the weak interaction, which occurs between all particles.

When a $W^{+}$or $W^{-}$boson is exchanged, the interaction is a charged-current interaction because charge is exchanged between the two interacting particles. When the $Z^{0}$ boson is exchanged, the interaction is a neutral-current interaction. The basic charged- and neutral-current interactions are shown in Fig. 2.1 and Fig. 2.2. Neutrinos only interact through the weak interaction.

In 1967, Glasgow, Weinberg, and Salaam developed a theory which united the weak and electromagnetic interactions [13] [14]. This theory claims that at high equilibrium temperatures these two interactions originate from the same interaction, the electroweak interaction. They differ today because of spontaneous symmetry breaking which occurred as the early universe cooled. Since neutrinos interact through the weak interaction, the study of neutrino interactions is a study of the electroweak interaction.

The standard model has been tested extensively using a variety of experiments. While 


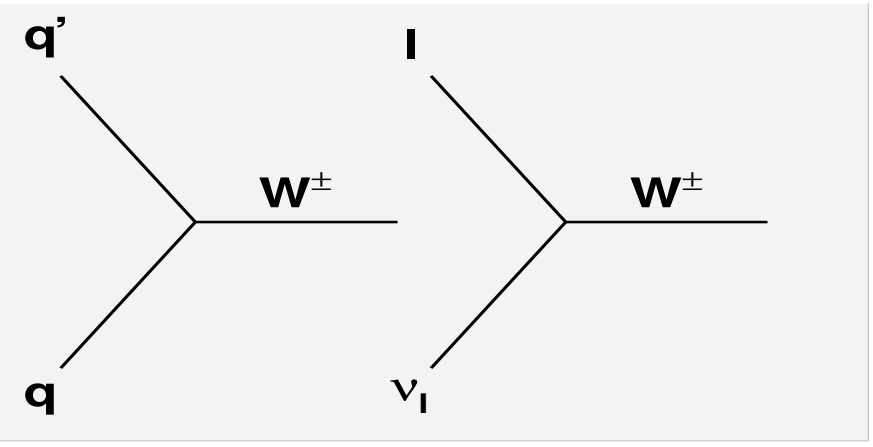

Figure 2.1: The basic interactions of the charged-current weak interaction. The left figure illustrates a quark emitting a $W^{ \pm}$boson; the right figure illustrates a lepton emitting a $W^{ \pm}$boson.

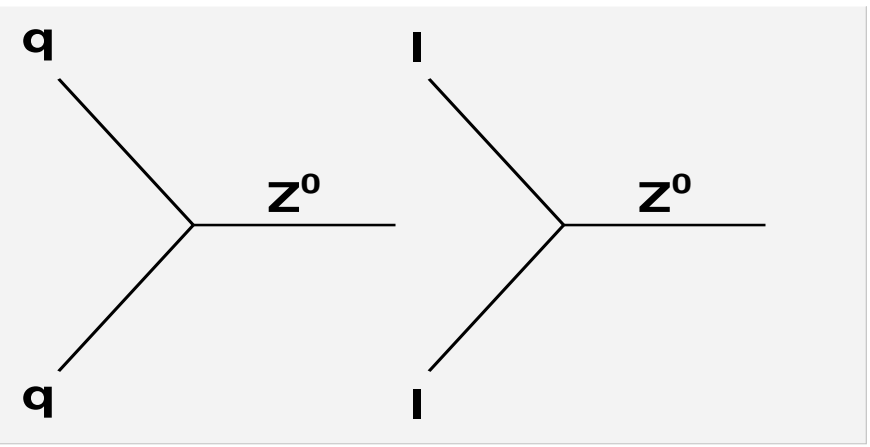

Figure 2.2: The basic interactions of the neutral-current weak interaction. The left figure illustrates a quark emitting a $Z^{0}$ boson; the right figure illustrates a lepton emitting a $Z^{0}$ boson. 
it has been very successful in explaining the experimental results, it is not a complete theory. The standard model requires arbitrary parameters; it cannot predict the masses of the fundamental particles. Gravity, the fourth fundamental interaction, is not included in the standard model. The standard model cannot explain the dark matter problem. Neutrino oscillation are not explained by the standard model. The standard model requires extensions to explain these and other issues. Tests of the standard model, such as the measurement of tau neutrino cross section, can provide information about these necessary extensions.

\subsection{Neutrino Interactions in the Standard Model}

Neutrino interactions are dominated by different mechanisms at different energy regions. At low energies, $100 \mathrm{MeV}-10 \mathrm{GeV}$, the interactions are almost all quasi-elastic. A quasi-elastic neutrino-nucleon interaction is modeled as a neutrino scattering off the entire nucleon. The cross section for quasi-elastic interactions is well measured and understood. At high energies, $20 \mathrm{GeV}-200 \mathrm{GeV}$, the interactions are in the deep inelastic scattering region. A deep inelastic scattering neutrino-nucleon interaction is modeled as a neutrino scattering off an essentially free quark inside the nucleon; the deep inelastic scattering cross sections are explained using the quark parton model. They are also well measured for the muon and electron neutrinos. At mid-energies, $\sim 10 \mathrm{GeV}$, the resonance production model is used. This region is difficult to model because both deep inelastic and quasi-elastic scattering occurs. The charged-current tau neutrino interactions in the DONUT experiment typically fell into the deep inelastic scattering region, as shown in the calculations in Appendix A.

Deep inelastic scattering is described using the following invariant quantities: $Q^{2}, W^{2}$, $x$, and $y . Q^{2}$ is defined as the square of the momentum transfer or the square of the invariant mass of the virtually exchanged boson. $W^{2}$ is the square of the four-momentum of the final state hadrons. $x$, the Bjorken-scaling parameter, is the fraction of momentum carried away by the struck quark. $y$ is the measure of the energy transferred between the lepton and hadron systems. These invariants are defined in terms of the four-momenta: $k, k^{\prime}$, and $P$, which are shown in Fig. 2.3. $k$ is the four-momentum of the neutrino; $k^{\prime}$ is the four-momentum of the lepton; $P$ is the four-momentum of the nucleon. The invariants are defined as follows:

$$
Q^{2}=-q^{2} \equiv-\left(k-k^{\prime}\right)^{2}=-m_{\tau}^{2}+2 E_{\nu_{\tau}}\left(E_{\tau}-p_{\tau} \cos \theta\right)
$$




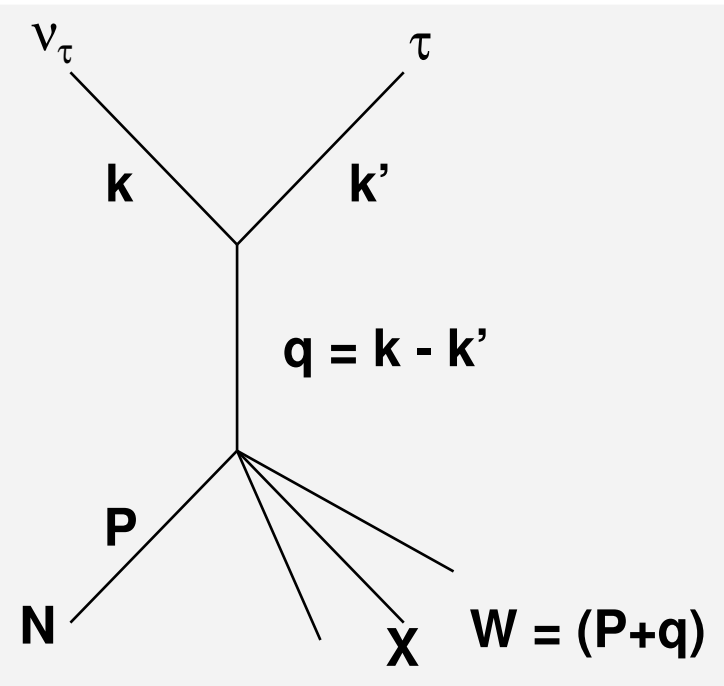

Figure 2.3: The Feynman diagram for a tau neutrino-nucleon interaction. $k$ is the fourmomentum of the tau neutrino, $k^{\prime}$ of the tau lepton, $P$ of the nucleon, and $W$ of the final state hadrons.

$$
\begin{aligned}
W^{2} & \equiv(P+q)^{2}=M^{2}-Q^{2}-2 M\left(E_{\nu_{\tau}}-E_{\tau}\right), \\
y & \equiv \frac{q \cdot p}{k \cdot p}=\frac{E_{\text {quarks }}}{E_{\nu}}, \text { and } \\
x & \equiv \frac{-q^{2}}{2 q \cdot p}=\frac{Q^{2}}{2 M E_{\text {quarks }}},
\end{aligned}
$$

where $m_{\tau}$ is the mass of the tau lepton, $E_{\nu_{\tau}}$ is the energy of the tau neutrino, $E_{\tau}$ is the energy of the tau lepton, $E_{\text {quarks }}$ is the energy of the quarks, $p_{\tau}$ is the momentum of the tau neutrino, $\theta$ is the angle between the tau neutrino and the tau lepton, and $M$ is the mass of the nucleon.

The differential neutrino-nucleon interaction cross section in the deep inelastic scattering region in terms of $Q^{2}, x$, and $y[15]$ is

$$
\begin{aligned}
\frac{d^{2} \sigma_{\nu N}^{\mathrm{SM}}}{d x d y} & =\left(\frac{M_{W}^{2}}{M_{W}^{2}+Q^{2}}\right)^{2} \frac{G^{2} M E_{\nu}}{\pi}\left[\left(1-y-\frac{M x y}{2 E_{\nu}}\right) F_{2}\left(x, Q^{2}\right)\right. \\
& +y^{2} x F_{1}\left(x, Q^{2}\right) \pm\left(y-\frac{y^{2}}{2}\right) x F_{3}\left(x, Q^{2}\right) \\
& \left.+\frac{m_{\tau}^{2}}{M E_{\nu}}\left(-F_{2}\left(x, Q^{2}\right)\left(\frac{M}{4 E_{\nu}}+\frac{1}{2 x}\right)+\frac{F_{1}\left(x, Q^{2}\right) y}{2} \mp \frac{F_{3}\left(x, Q^{2}\right) y}{4}\right)\right],
\end{aligned}
$$


where $G$ is Fermi coupling constant and $F_{i}^{\nu N}(x)$ are the dimensionless structure functions which describe the distribution of momentum among the quarks inside the nucleon. All terms proportional to the lepton and nucleon masses, negligible in the case of the muon and electron neutrino cross sections, are included here. The upper sign is for the neutrino cross section; the lower sign is for the anti-neutrino cross section. Both will be denoted as $\sigma^{\nu N}$ for ease of notation. If $Q^{2}<<M_{W}$,

$$
\frac{M_{W}^{2}}{M_{W}^{2}+Q^{2}} \cong 1
$$

which is true for the DONUT experiment. For inelastic scattering, the structure functions are weakly dependent on $Q^{2}$. If $Q^{2}>M$, the structure functions are approximately functions of $x$ and not $Q^{2}[16]$; therefore,

$$
F_{i}\left(x, Q^{2}\right) \cong F_{i}(x)
$$

This behavior was first witnessed in early inelastic scattering experiments performed at the Stanford Linear Accelerator (SLAC) [17] in 1968; this behavior is known as Bjorken scaling and is consistent with the lepton scattering off point-like particles, partons, in the nucleon [18]. If these partons are spin $1 / 2$ particles, such as quarks or anti-quarks, the Callan-Gross relation [19] gives

$$
2 x F_{1}(x)=F_{2}(x) .
$$

Using Eq. 2.2, Eq. 2.3, Eq. 2.4, and 2.5, the cross section is

$$
\begin{aligned}
\frac{d^{2} \sigma_{\nu N}^{\mathrm{SM}}}{d x d y} & =\frac{G^{2} M E_{\nu}}{\pi}\left[F_{2}(x)\left(1-y-\frac{M x y}{2 E_{\nu}}+\frac{y^{2}}{2}-\frac{m_{\tau}^{2}}{4 E_{\nu}^{2}}-\frac{m_{\tau}^{2}}{2 x M E_{\nu}}+\frac{m_{\tau}^{2} y}{4 x M E_{\nu}}\right)\right. \\
& \left.+F_{3}(x)\left( \pm x y \mp \frac{y^{2} x}{2} \mp \frac{m_{\tau}^{2} y}{4 M E_{\nu}}\right)\right] .
\end{aligned}
$$

Using the parton model, the structure functions are related to the parton density functions $[16]$,

$$
\begin{aligned}
F_{2}^{\nu N}(x) & =2 x(q(x)+\bar{q}(x)), \text { and } \\
x F_{3}^{\nu N}(x) & =2 x(q(x)-\bar{q}(x)) .
\end{aligned}
$$

The notation of the literature uses $q(x)$ to represent the parton density function; this $q_{i}(x)$ is different than the $q$ used above for momentum transfer. The parton density functions are probabilities that the quark that was struck by the neutrino carries a fraction $x$ of the 
hadron's total momentum. For an isoscalar target, one with an equal number of protons and neutrons, the average quark and anti-quark distributions are

$$
\begin{aligned}
& q(x)=\frac{u(x)+d(x)}{2}, \text { and } \\
& \bar{q}(x)=\frac{\bar{u}(x)+\bar{d}(x)}{2} .
\end{aligned}
$$

Using Eq. 2.7, the cross section is

$$
\begin{aligned}
\frac{d^{2} \sigma_{\nu N}^{\mathrm{SM}}}{d x d y} & =\frac{G^{2} M E_{\nu}}{\pi}\left[x q\left(2-2 y(1 \mp 1)+y^{2}\left(1 \mp 1-\frac{m_{\tau}^{2}}{2 E_{\nu}^{2}}\right)\right)\right. \\
& +x \bar{q}\left(2-2 y(1 \pm 1)+y^{2}(1 \pm 1)-\frac{m_{\tau}^{2}}{2 E_{\nu}^{2}}\right)+q \frac{m_{\tau}^{2}}{M E_{\nu}}\left(-1+\frac{y}{2} \mp \frac{y}{2}\right) \\
& +\bar{q} \frac{m_{\tau}^{2}}{M E_{\nu}}\left(-1+\frac{y}{2} \pm \frac{y}{2}\right)-x^{2} q \frac{M y}{E_{\nu}}-x^{2} \bar{q} \frac{M y}{E_{\nu}}
\end{aligned}
$$

The limits of $x$ and $y$ come from the deep inelastic scattering kinematic limits on $Q^{2}$ and $W^{2}[20]$. They are

$$
\begin{gathered}
\frac{m_{\tau}^{2}}{2 M\left(E_{\nu}-m_{\tau}\right)} \leq x \leq 1, \text { and } \\
a-b \leq y \leq a+b,
\end{gathered}
$$

where

$$
a=\frac{1-m_{\tau}^{2}\left(\frac{1}{2 M E_{\nu} x}+\frac{1}{2 E_{\nu}^{2}}\right)}{2\left(1+\frac{M x}{2 E_{\nu}}\right)}
$$

and

$$
b=\frac{\sqrt{1-\left(\frac{m_{\tau}^{2}}{2 M E_{\nu} x}\right)-\frac{m_{\tau}^{2}}{E_{\nu}^{2}}}}{2\left(1+\frac{M x}{2 E_{\nu}}\right)} .
$$

The forms of the parton density functions, PDFs, are not determined by QCD, although QCD does provide overall constraints and the evolution in $Q^{2}$. Eventually lattice gauge theory may provide estimates for the PDFs. Currently they are estimated using data and perturbative QCD. Several groups produce sets of PDFs. The results from the 
CTEQ group [21], which calculated several different sets extracted under slightly different conditions, are used in this calculation. The PDFs are complicated functions of energy. As a consistency check, I used the QCD constraints, given below, to check the PDFs at a neutrino energy of $115 \mathrm{GeV}$, the average interacted tau neutrino energy in the DONUT experiment. Eq. 2.14 summarizes the relevant parton density functions from the CTEQ6M version for neutrinos with energy $115 \mathrm{GeV}$ integrated using the limits for $x$ described in Eq. 2.13 and Eq. 2.14. This calculation was performed using Mathematica [22] and CTEQ programs [21]. Fig. 2.4 illustrates the parton density functions for the $u$ and $d$ quarks for a neutrino energy of $115 \mathrm{GeV}$.

$$
\begin{array}{cc}
\int u(x) d x=19.0 \pm 1.0 & \int \bar{u}(x) d x=17.0 \pm 0.9 \\
\int d(x) d x=17.8 \pm 0.9 & \int \bar{d}(x) d x=16.8 \pm 0.9 \\
\int x u(x) d x=0.28 \pm 0.01 & \int x \bar{u}(x) d x=0.04 \pm 0.001 \\
\int x d(x) d x=0.13 \pm 0.003 & \int x \bar{d}(x) d x=0.04 \pm 0.001 \\
\int x^{2} u(x) d x=0.07 \pm 0.003 & \int x^{2} \bar{u}(x) d x=0.003 \pm 0.0001 \\
\int x^{2} d(x) d x=0.003 \pm 0.0001 & \int x^{2} \bar{d}(x) d x=0.005 \pm 0.0002
\end{array}
$$

The errors for these distributions are the uncertainties in the theoretical models and in the method of extracting the PDFs from these models. These errors are complicated, but a simple estimate of the errors is obtained by comparing the PDFs from different versions of the CTEQ fits; this is the origin of the errors quoted Eq. 2.14.

As previously mentioned, while QCD does not determine the forms of the PDF sets, it does provide overall constraints. The sum rules, which are a consequence of the conservation of quantum numbers, are one set of constraints. The sum rules are

$$
\begin{array}{r}
\int(u(x)-\bar{u}(x)) d x=2, \text { and } \\
\int(d(x)-\bar{d}(x)) d x=1 .
\end{array}
$$

It is impossible to directly verify these rules experimentally, but different sum rules based on these can be tested. The Gross Llewellyn-Smith (Eq. 2.16) [17] and the Alder (Eq. 


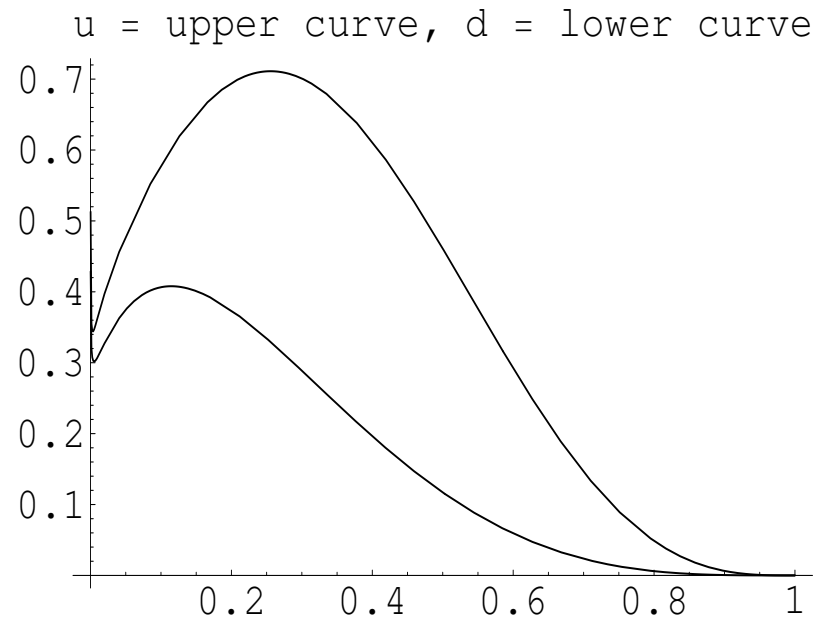

Figure 2.4: The parton distribution functions for the $u$ and $d$ quarks for $E_{\nu}=115 \mathrm{GeV}$. The $\mathrm{x}$-axis is the Bjorken $x$, and the $\mathrm{y}$-axis is $u(x)$ or $d(x)$.

2.17) [23] sum rules

$$
\begin{gathered}
\int(u(x)-\bar{u}(x)+d(x)-\bar{d}(x)) d x=3, \text { and } \\
\int(u(x)-\bar{u}(x)-d(x)+\bar{d}(x)) d x=1
\end{gathered}
$$

were verified experimentally using early neutrino data on isoscalar targets [24]. As a consistency check, the sum rules are calculated using the CTEQ PDFs at a neutrino energy of $115 \mathrm{GeV}$

$$
\begin{array}{r}
\int(u(x)-\bar{u}(x)) d x=1.99 \pm 0.15, \text { and } \\
\int(d(x)-\bar{d}(x)) d x=1.00 \pm 0.07 .
\end{array}
$$

The CTEQ PDF sets satisfy the sum rules. Another constraint on the PDF sets involves momentum conservation. The sum of the momentum of the partons must equal the total momentum of the nucleon. Experiments show that the total momentum of the valence quarks constitutes approximately half of the total nucleon momentum [19]; therefore,

$$
\int x(u(x)+d(x)+\bar{u}(x)+\bar{d}(x)) d x \sim 0.5 .
$$


The CTEQ PDF sets for $115 \mathrm{GeV}$ neutrinos yield

$$
\int x(u(x)+d(x)+\bar{u}(x)+\bar{d}(x)) d x=0.488 \pm 0.12,
$$

satisfying this constraint.

Using the PDF set from CTEQ, the tau neutrino and anti-neutrino charged-current cross sections divided by energy are calculated as a function of energy. The result is shown in Fig. 2.5. The same calculations were done for the muon neutrino and anti-neutrino, shown in Fig. 2.6, and are compared to the experimental result, shown in Fig. 2.7. As seen in these figures, the calculations are in good agreement with the measurements of the muon neutrino and anti-neutrino cross sections.

At the average energy of the interacted tau neutrinos, $115 \mathrm{GeV}$, the theoretical tau neutrino and anti-neutrino charged-current cross sections are

$$
\begin{gathered}
\sigma_{\nu_{\tau} N}^{\mathrm{SM}}=66 \pm 5 \times 10^{-38} \mathrm{~cm}^{2}, \text { and } \\
\sigma_{\bar{\nu}_{\tau} N}^{\mathrm{SM}}=29 \pm 2 \times 10^{-38} \mathrm{~cm}^{2} .
\end{gathered}
$$

Since an equal number of tau neutrinos and tau anti-neutrinos exist in the beam, the measured cross section is the average,

$$
\sigma_{\nu_{\tau} N}^{\mathrm{SM}}=48 \pm 5 \times 10^{-38} \mathrm{~cm}^{2} .
$$

If the standard model accurately describes the interaction of the tau neutrino; then the charged-current cross section measurement should agree with Eq. 2.23 and Fig. 2.5 within errors. 


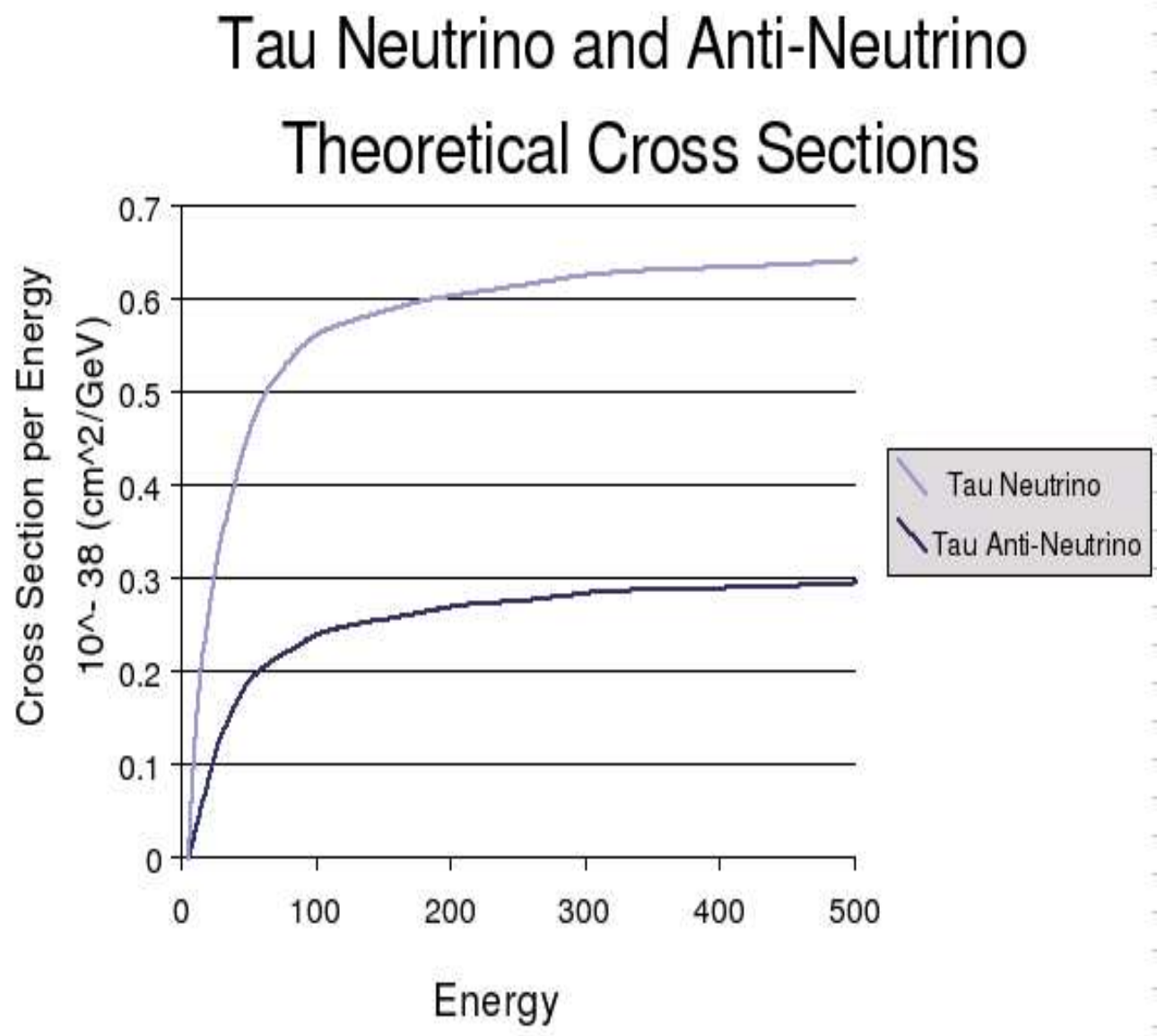

Figure 2.5: The theoretical tau neutrino and anti-neutrino charged-current cross sections divided by energy as a function of energy. 


\section{Muon Neutrino and Anti-Neutrino Theoretical Cross Sections}

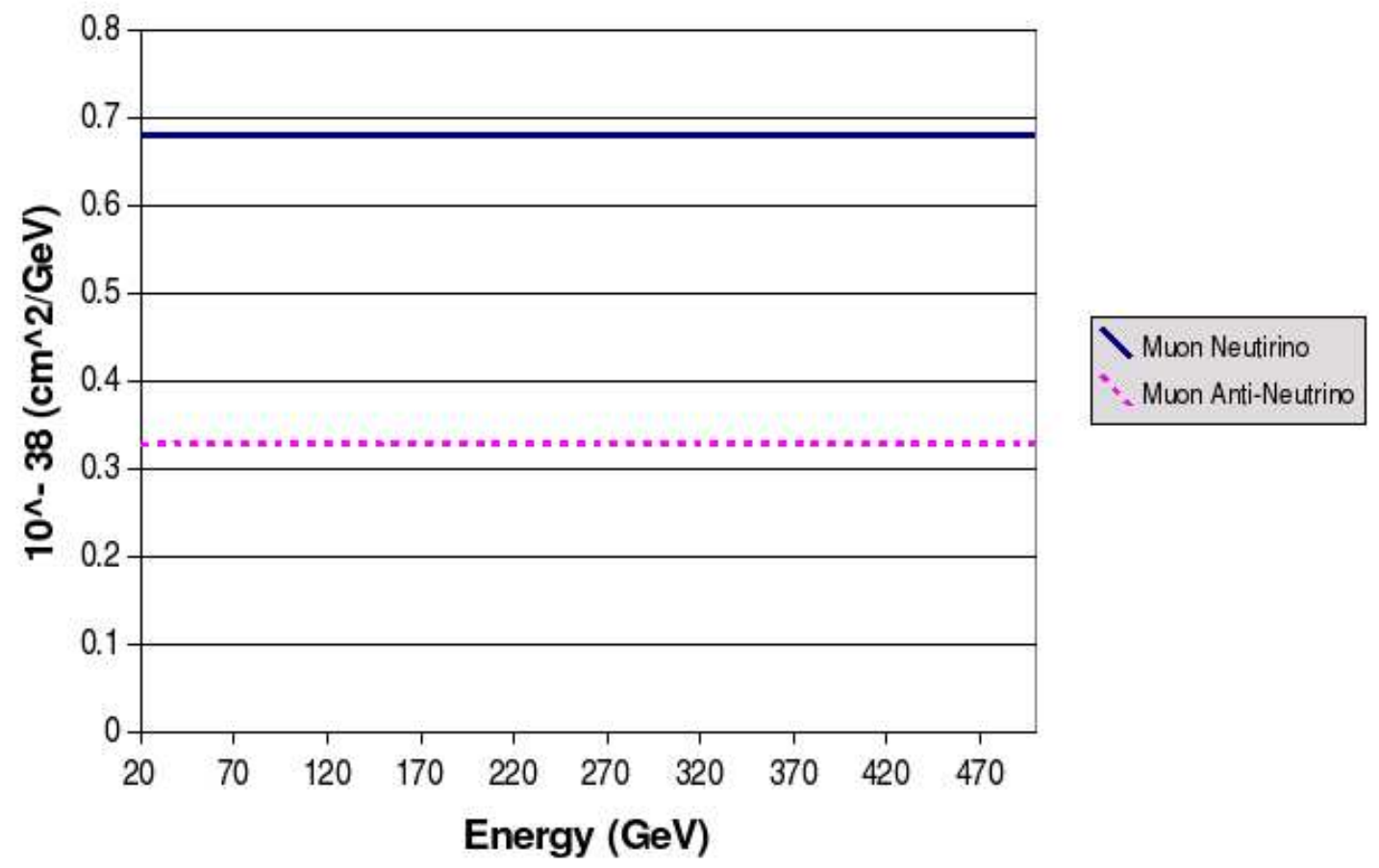

Figure 2.6: The theoretical muon neutrino and anti-neutrino charged-current cross sections divided by energy as a function of energy, where

$$
\begin{gathered}
\frac{\sigma_{\nu \mu}^{\mathrm{SM}}}{E}=0.678 \pm 0.05 \times 10^{-38} \mathrm{~cm}^{2} \mathrm{GeV}^{-1} \text { and } \\
\frac{\sigma_{\bar{\nu} \mu}^{\mathrm{SM}}}{E}=0.327 \pm 0.02 \times 10^{-38} \mathrm{~cm}^{2} \mathrm{GeV}^{-1}
\end{gathered}
$$




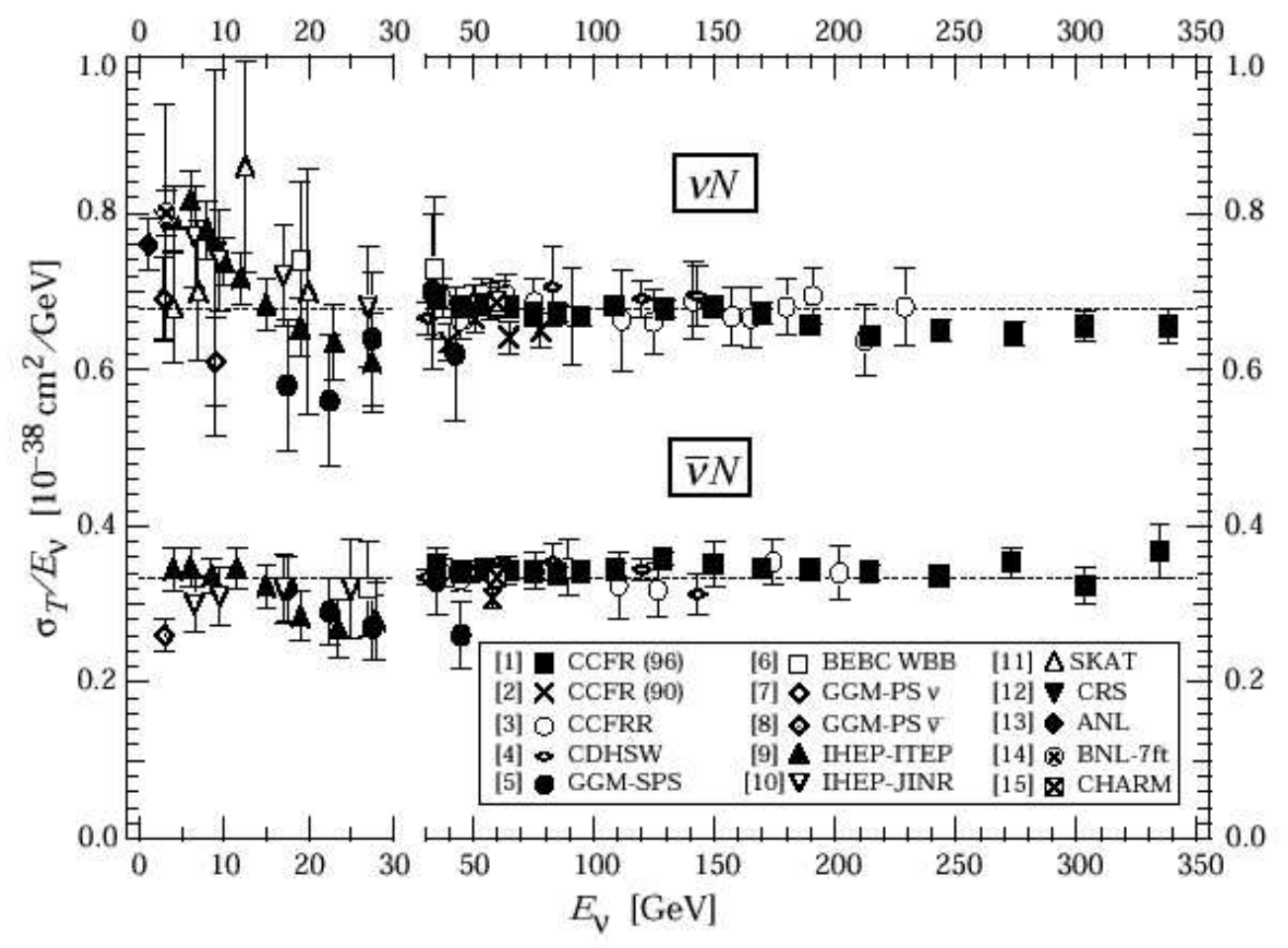

Figure 2.7: Experimental muon neutrino and anti-neutrino cross sections divided by energy [25]. The lines represent the best fit, which are

$$
\begin{gathered}
\frac{\sigma_{\nu \mu}^{\exp }}{E}=0.677 \pm 0.014 \times 10^{-38} \mathrm{~cm}^{2} \mathrm{GeV}^{-1} \text { and } \\
\frac{\sigma_{\bar{\nu} \mu N}^{\exp }}{E}=0.334 \pm 0.008 \times 10^{-38} \mathrm{~cm}^{2} \mathrm{GeV}^{-1} .
\end{gathered}
$$




\section{Chapter 3}

\section{The Experimental Setup}

\subsection{Introduction}

When a tau neutrino interacts through the charged-current interaction, a tau lepton is produced. Subsequently, the tau lepton decays, typically within $2 \mathrm{~mm}$ in this experiment. To identify the tau neutrino interaction, the tau lepton decay must be observed. Since this requires a high-resolution detector, nuclear emulsion was used. Other components of the experiment were designed to complement the nuclear emulsion detector and are explained in Ref. [26].

Emulsion is a permanently active detector, recording every charged particle traversing it. Without shielding to reduce the charged particle rate through the experiment, the emulsion would have been useless after one pulse. The shielding consisted of two magnets that deflected charged particles and concrete and iron that degraded the energy of particles and absorbed low energy particles. The shielding was placed between the production target and the emulsion. The shielding configuration is shown in Fig. 3.2.

The DONUT experiment was designed to observe tau lepton decays in the emulsion. Since digitizing all emulsion data would take on the order of years, a hybrid emulsion detector was used to locate a small volume of emulsion in which the neutrino interaction took place. This detector consisted of modules made of emulsion, iron, and plastic, interleaved with scintillating fibers, as shown in Fig. 3.3. The scintillating fibers were used to reconstruct the tracks of charged particles produced in the neutrino interactions and, thus, identify the region of emulsion to be examined. A volume of $5 \times 5 \times 10 \mathrm{~mm}^{3}$, centered on the predicted location of the neutrino interaction, was digitized and pattern recognition software, assisted by humans, located the charged tracks coming from the neutrino 
interaction. To differentiate the muon neutrino and electron neutrino charged-current interactions from tau neutrino charged-current interactions, a downstream spectrometer, shown in Fig. 3.5, identified muons and electrons. The spectrometer also measured the momentum of the charged tracks.

\subsection{The Neutrino Beam}

$800 \mathrm{GeV}$ protons from the Tevatron, a particle accelerator at Fermilab National Accelerator Laboratory, were guided onto a tungsten target to produce particles that decayed into tau neutrinos. The beam incident on the production target consisted of approximately $8 \times 10^{12}$ protons per spill with a twenty second spill each minute.

\subsubsection{The Tungsten Target}

Tungsten was used as a target because of its high density. The target was a square $10 \mathrm{~cm}$ x $10 \mathrm{~cm}$ in cross section and $102 \mathrm{~cm}$ long. Surrounding the target, a copper sheath with water flowing through it cooled the target. Since the target was 10.6 nuclear interaction lengths, virtually all protons interacted within the target.

The particles of interest in this experiment were the charm mesons and charm baryons, which each produced neutrinos through their decays. The charm mesons, short-lived particles with a lifetime on the order of $10^{-12} \mathrm{~s}$, typically decayed before interacting. When a charm particle decayed semi-leptonically and produced a tau neutrino, a tau lepton was also produced. The only mesons produced in this experiment that were massive enough to produce a tau lepton were the $D_{s}$ particle and the $D^{ \pm}$. The $D_{s}$ meson, slightly heavier than the $D^{ \pm}$meson, was the only significant source of tau neutrinos. The decay of the $D_{s}^{+}\left(D_{s}^{-}\right)$produced two tau neutrinos; the first was a tau neutrino (tau anti-neutrino) produced in the initial $D_{s}$ decay, and the second was a tau anti-neutrino (tau neutrino) which resulted from the subsequent tau (anti-tau) lepton decay. The decay of the $D_{s}^{+}$ particle is shown in Fig. 3.1.

Electron and muon neutrinos were also produced in the decay of charm particles, specifically the $D^{ \pm}, D^{0}, D_{s}$, and $\Lambda_{c}$. Excited states of the charm particles, such as $D^{* \pm}$ and $D^{* 0}$, also contributed as they quickly decayed into other charm particles with the following ratios [30]:

$$
\begin{array}{lll}
D^{* \pm} \rightarrow D^{ \pm} \pi^{0}, D^{ \pm} \gamma & 31.7 \% \\
D^{* \pm} \rightarrow D^{0} \pi^{ \pm} & 68.3 \%
\end{array}
$$




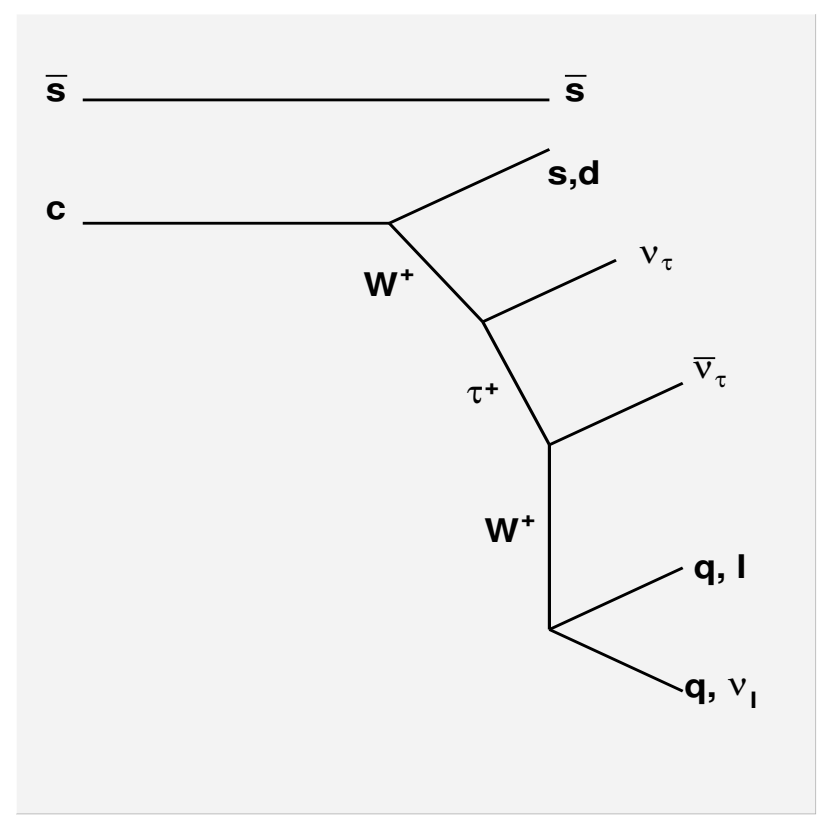

Figure 3.1: The $D_{s}^{+}$decay.

$$
\begin{array}{rlr}
D^{* 0} & \rightarrow D^{ \pm} X & 0 \% \\
D^{* 0} \rightarrow D^{0} \pi^{0}, D^{0} \gamma & 100 \%
\end{array}
$$

The decay of light mesons, such as pions and kaons, produced muon neutrinos and electron neutrinos. Approximately half of the muon neutrinos [28] were produced in the decays of light mesons; virtually none of the electron neutrinos came from light meson decays because the decay through the electron mode was highly suppressed due to its small mass.

Using measured production cross sections and branching ratios, together with the energy spectrum of the muons in this experiment, it was calculated that the beam of neutrinos consisted of $5 \%$ tau neutrinos, $47 \%$ muon neutrinos, of which $49 \%$ were prompt and $51 \%$ were non-prompt [28], and $48 \%$ electron neutrinos [29].

\subsubsection{Shielding}

The track density in the emulsion was limited to $5 \times 10^{5}$ tracks per $\mathrm{cm}^{3}$; this limit was determined from prior experiments [27]. If the track density was higher than this limit, 
the emulsion would have been too dense to resolve individual events using the automated scanning technique, described in a later section. Without shielding, the charged particle density at the point of the emulsion detector would have been $10^{12}$ tracks per $\mathrm{cm}^{3}$. Even with the shielding, the largest source of charged particles in the emulsion detector were penetrating muons from the beam dump. Thermal neutrons and gamma rays originated in material surrounding the experiment. The shielding configurations is shown in Fig. 3.2.

The active shielding, composed of two large electromagnets, was placed after the beam dump. The first magnet deflected most high energy muons away from the emulsion. The second magnet deflected any high energy particles that passed through the first magnet or were bent back into the beam by the first magnet. The first magnet was a $7.4 \mathrm{~m}$ dipole magnet with a vertical field of $3.0 \mathrm{~T}$. The second magnet was $5 \mathrm{~m}$ long, made of iron, and had a vertical field of $1.9 \mathrm{~T}$. Together these magnets deflected most high energy muons into two plumes separated by $2 \mathrm{~m}$ at the position of the emulsion target. One of the plumes is shown in Fig. 3.2. The magnets decreased the muon rate from $6 \mathrm{kHz}$ per $\mathrm{cm}^{2}$, as measured $1.5 \mathrm{~m}$ off center of emulsion, to $0.2 \mathrm{kHz}$ per $\mathrm{cm}^{2}$, the average flux measured in the emulsion.

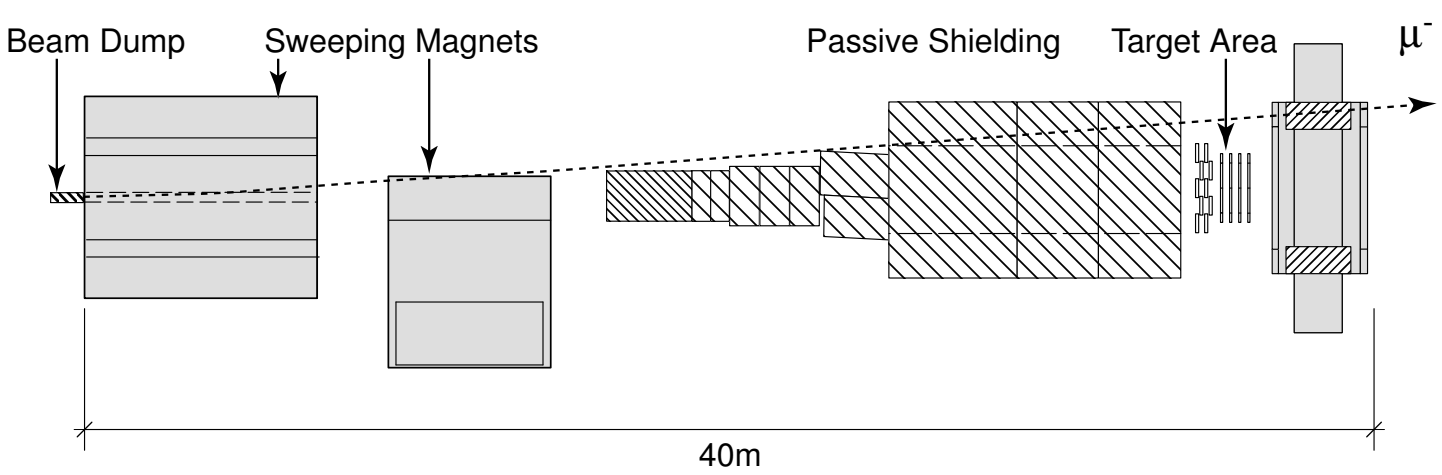

Figure 3.2: Overview of Shielding [27]. The beam dump is shown at the left of this figure. After the beam dump was the active shielding which consisted of two magnets that swept charged particles, mostly muons, away from the emulsion target into two plumes. One of the plumes is represented as a dashed line that begins in the beam dump and is deflected by the magnet. The plume, which is labeled as $\mu^{-}$, misses the target area. The passive shielding which followed consisted of seventeen meters of iron and concrete where most of the low momentum muons interacted through ionization and thermal neutrons and gamma rays were absorbed. 
The passive shielding consisted of 17 meters of iron and concrete, where most of the low momentum muons interacted through ionization. The steel absorbed gamma rays; the concrete absorbed the neutrons. No steel was in the path of the muon plumes to avoid more charged particles from secondary interactions.

\subsection{The DONUT Detector}

The tau lepton decay is the signature of a tau neutrino charged-current interaction. Approximately $86 \%$ [25] of these tau lepton decays result in one charged particle plus neutral particles; the topology of this decay is a short track with a kink. $14 \%$ of tau lepton decays result in three charged particles plus neutral particles. The DONUT detector was optimized to observe both types of decays.

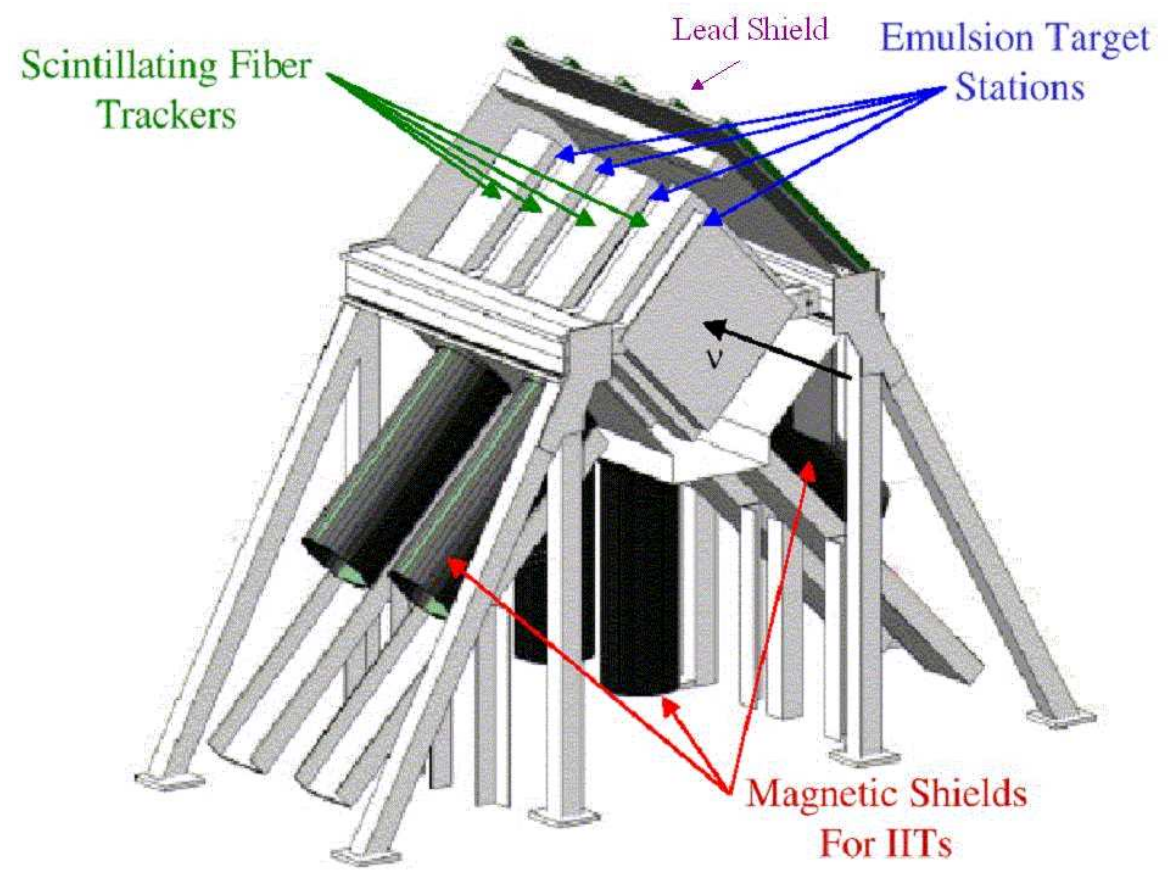

Figure 3.3: The emulsion detector [29]. The emulsion modules were interleaved with scintillating fiber tracker stations. Since a magnet directly follows the detector, pipes were placed around the IITs to shield them from fringe fields. A $20 \mathrm{~mm}$ thick lead shield surrounded the target. 


\subsubsection{The Emulsion Target}

Emulsion was used because of its good spacial and angular resolution, which was required to observe the tau lepton decay. Modules made of emulsion, plastic, and iron were interleaved with scintillating fibers, arranged in scintillating fiber tracker, SFT, stations as shown in Fig. 3.3. At most, four emulsion modules were exposed at a time, with some periods only exposing two. A total of seven modules was exposed for approximately one month each.

Emulsion is similar to photographic film. It consisted of silver bromide, $\mathrm{AgBr}$, grains suspended in gelatin deposited on a layer of acrylic plastic. The emulsion was mixed and deposited by the experimenters. As charged particles traversed the emulsion, they ionized the $\mathrm{AgBr}$ grains, changing the $\mathrm{AgBr}$ grains's chemical makeup, resulting in a darker appearance after development. These ionized grains were used to reconstruct the paths of charged particles traversing the emulsion.

An emulsion module was a $50 \times 50 \times 7 \mathrm{~cm}^{3}$ container filled with stacked sheets of emulsion. Three different emulsion sheet configurations were used. Fig. 3.4 illustrates these configurations. The first two types of emulsion sheets had a similar structure. The ECC 200 (Fig. 3.4 (a)) and the ECC 800 (Fig. 3.4 (b)) consisted of either a 200 or $800 \mu \mathrm{m}$ plastic base coated on either side with a $100 \mu \mathrm{m}$ layer of emulsion and a $1 \mathrm{~mm}$ sheet of iron on one side. In this configuration, the iron sheets provided most of the mass. The ECC configurations were sampling detectors which provided the position and angle of particle tracks at discrete points. These "track segments" were used to reconstruct the track. The disadvantage of the sampling detector was the possibility that the tau lepton decayed before it exited the iron. The last configuration, Fig. 3.4 (c), was the bulk emulsion sheet, which had a $350 \mu \mathrm{m}$ layer of emulsion on each side of a $90 \mu \mathrm{m}$ base of plastic. The bulk configuration was the most expensive type of emulsion configuration because emulsion accounted for $95 \%$ of the mass. The bulk configuration acted as a volume detector; when a neutrino interacted in the bulk emulsion, the entire tau lepton track was observed. The seven modules exposed during this experiment consisted of either 80 bulk emulsion sheets, 47 ECC 200 emulsion sheets, or a combination of bulk, ECC 200, or ECC 800 emulsion sheets. Table 3.1 summarizes the details of the configurations and masses for each module. In addition, changeable sheets were placed on the front and back of every module; these were replaced weekly. 


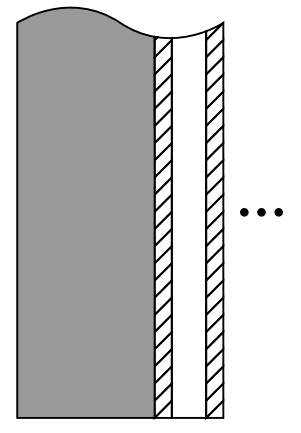

(a)

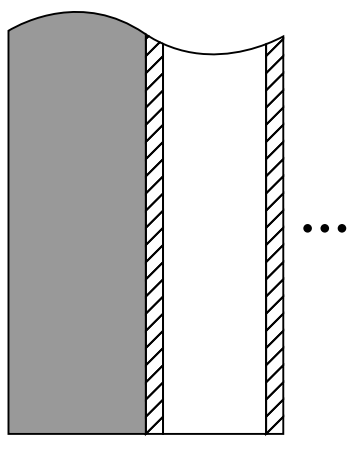

(b)

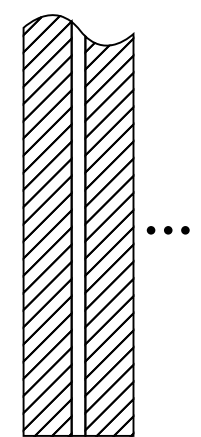

(c)

Figure 3.4: Three emulsion sheet configurations [26]. The hatched represents the emulsion, the gray represents the steel, and white represents the plastic. (a) The ECC 200 configuration consisted of $1 \mathrm{~mm}$ of iron and $200 \mu \mathrm{m}$ of plastic with $100 \mu \mathrm{m}$ of emulsion on either side. (b) The ECC 800 configuration was similar to (a) except it used $800 \mu \mathrm{m}$ of plastic. (c) The bulk configuration consisted of $350 \mu \mathrm{m}$ of emulsion on either side of a $90 \mu \mathrm{m}$ plastic base.

\section{Composition of Emulsion Modules}

\begin{tabular}{|c|c|c|c|c|}
\hline Module & $\begin{array}{l}\text { Sheet Composition } \\
\text { (Number of sheets) }\end{array}$ & Period & Station & $\begin{array}{c}\text { Mass }(\mathrm{kg}) \\
\text { ECC/Bulk/Total }\end{array}$ \\
\hline ECC1 & (47) ECC200 & $1-3$ & 1 & 100.49 / 0 / 100.49 \\
\hline ECC3 & (47) ECC200 & $1-2$ & 3 & $100.49 / 0 / 100.49$ \\
\hline $\mathrm{E} / \mathrm{B} 1$ & (21) ECC800 (30) Bulk & 4 & 1 & $49.15 / 19.35 / 68.50$ \\
\hline $\mathrm{E} / \mathrm{B} 2$ & (19) ECC800 (38) Bulk & $3-4$ & 2 & $42.12 / 25.15 / 67.27$ \\
\hline $\mathrm{E} / \mathrm{B} 3$ & (20) ECC800 (32) Bulk & $3-4$ & 3 & $44.46 / 21.28 / 65.74$ \\
\hline E/B4 & $\begin{array}{c}\text { (2) ECC200 } \\
\text { (7) ECC } 800 \\
\text { (47) Bulk }\end{array}$ & $2-3$ & 4 & $36.71 / 27.73 / 64.44$ \\
\hline $\mathrm{B} 4$ & (87) Bulk & 4 & 4 & $0 / 56.10 / 56.10$ \\
\hline
\end{tabular}

Table 3.1: The composition of the seven emulsion modules. Each module was exposed for approximately one month. 


\subsubsection{The Spectrometer}

The spectrometer was used to record the charged tracks emerging from the emulsion for each event. This data was used to predict a vertex location within the emulsion, identify the charged particles resulting from the neutrino interactions, identify muons and electrons resulting from the muon neutrino interactions, electron neutrino interactions, and tau lepton decays, and measure the momentum of the charged particles. The spectrometer was $36 \mathrm{~m}$ downstream of the beam dump and consisted of five parts: the scintillating fiber tracks stations that resided in the target area, the scintillation counter trigger system, the momentum measurement system, the electromagnetic calorimeter, and the muon ID system. These components are shown in the Fig. 3.5

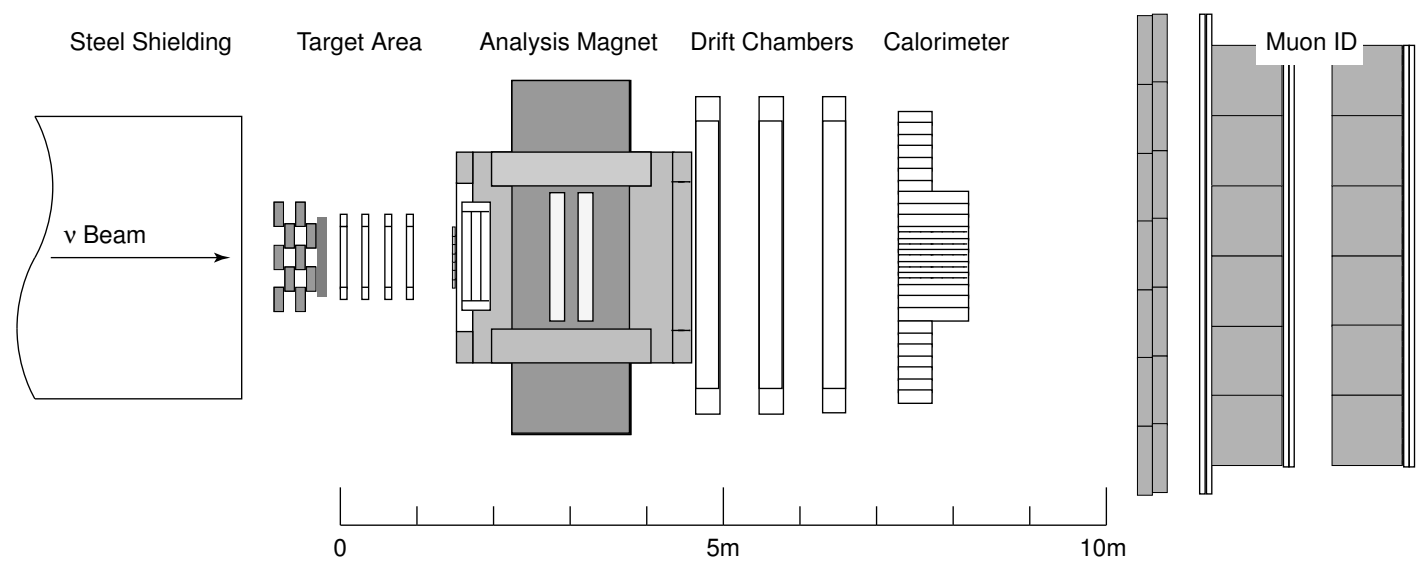

Figure 3.5: Overview of the DONUT spectrometer [27]. After traversing the shielding, the beam was incident on the target area where a small fraction of the neutrinos interacted. Charged particles from the neutrino interactions traveled through an analysis magnet and a set of drift chambers. The analysis magnet and drift chambers provided a momentum measurement of the charged tracks. The particles then traveled through the electromagnetic calorimeter which functioned as an electron ID. The final component of the spectrometer was the muon ID.

\section{The Scintillating Fiber Tracker Stations}

The scintillating fiber tracker stations were designed to reconstruct tracks of charged particles within a time window of $33 \mathrm{msec}$ of the trigger and to use these tracks to point to the location of the neutrino interaction in the emulsion module. The fibers were plastic 
fibers with polystyrene centers doped with $1 \%$ butyl-PBD and $0.1 \%$ BDB scintillator; they were $0.5 \mathrm{~mm}$ diameter. As charged particles passed through the fibers, they caused the scintillator to emit light. The scintillator trapped approximately $4 \%$ of the light, which traveled down the fiber to the end. One end of the fiber was aluminized and reflected the light; the other end was attached to the photo-cathode of the image intensifier to record the light signal. The difference in light output from ionizing particles from one end to the other due to fiber absorption was under $15 \%$ [28].

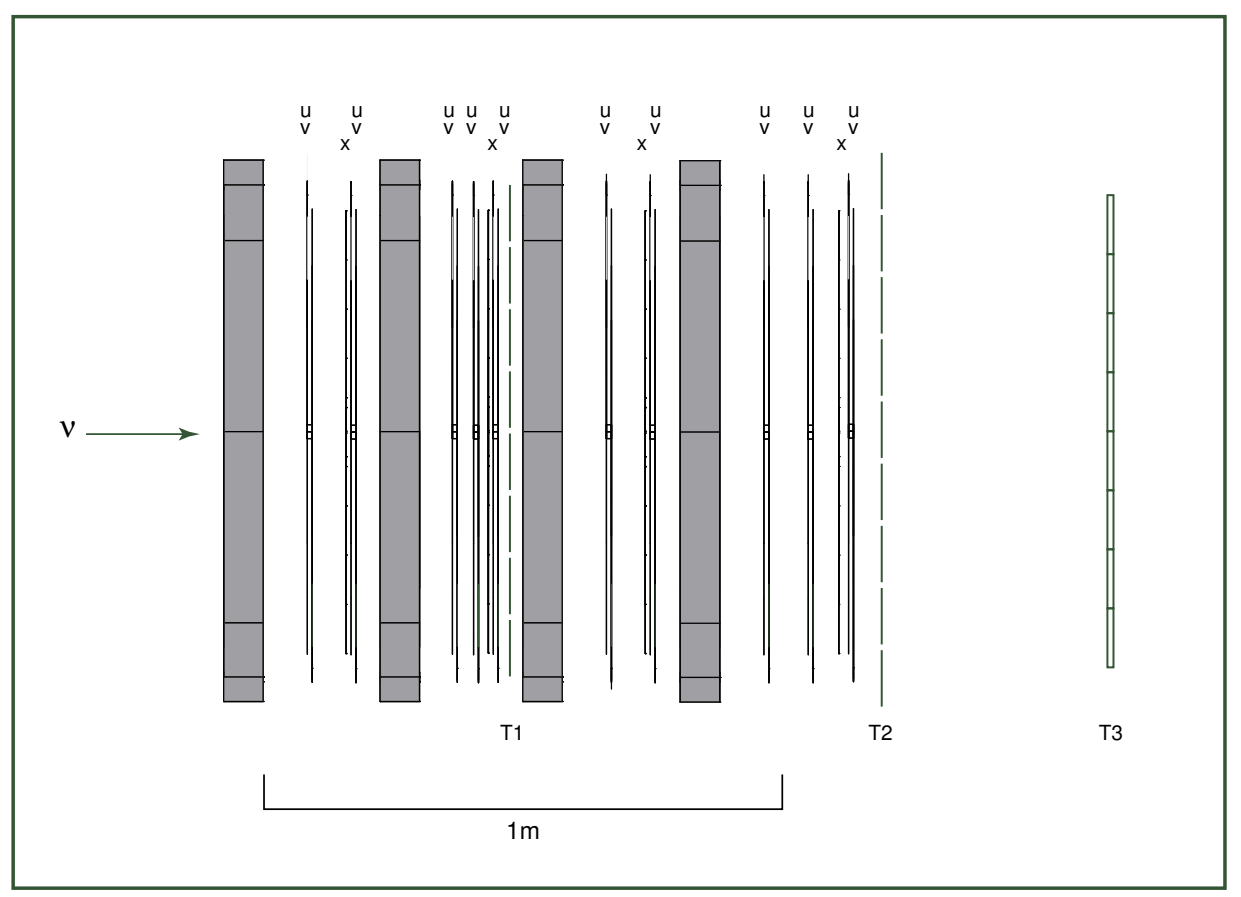

Figure 3.6: The scintillating fiber tracker stations [30].

60,000 fibers were placed in four stations between the emulsion modules; each station had nine to thirteen planes. A total of 44 planes were fixed together mechanically with an accuracy of $50 \mu \mathrm{m}[27]$. The fibers were placed side by side, arranged in layers. To increase light output, each layer was coated with $\mathrm{TiO}_{2}$ paint, which also served as glue to hold the fibers in place. Three different orientations of planes were used: the x-plane, which was six fibers thick and oriented vertically, the U-plane which was two fibers thick and oriented at $+45^{\circ}$, and the V-plane which was also two fibers thick and oriented at $-45^{\circ}$. The planes covered an area of $56 \mathrm{~cm} \times 56 \mathrm{~cm}$, slightly larger than the emulsion 
modules. Two types of stations existed: type I was located behind emulsion modules 2 and 4 and consisted of $1 \mathrm{X}$-plane, 3 U-planes, and $3 \mathrm{~V}$-planes and type II was located behind emulsion modules 1 and 3 and consisted of $1 \mathrm{X}$-plane, $2 \mathrm{U}$-planes, and $2 \mathrm{~V}$-planes. Both types are shown in Fig. 3.6.

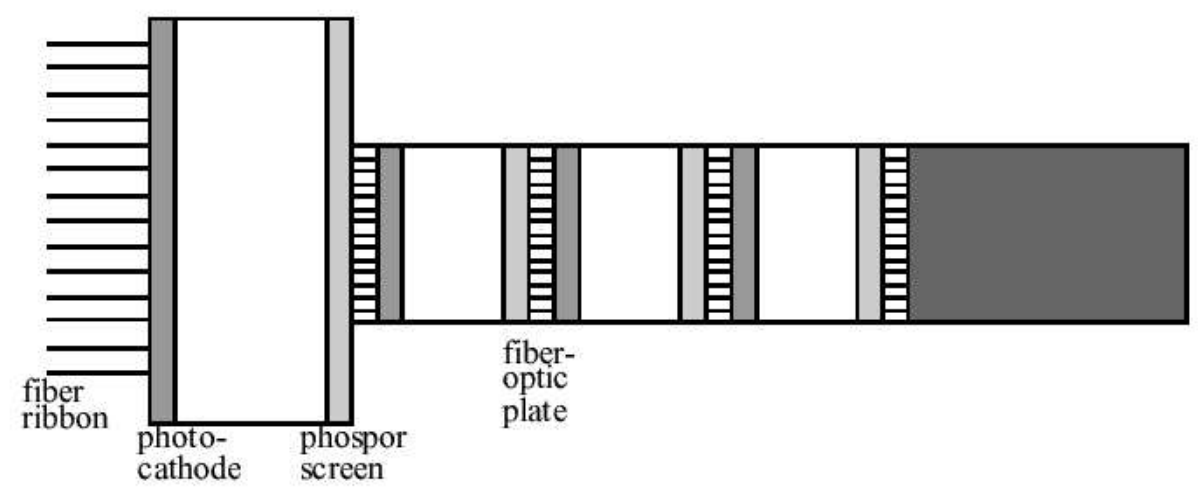

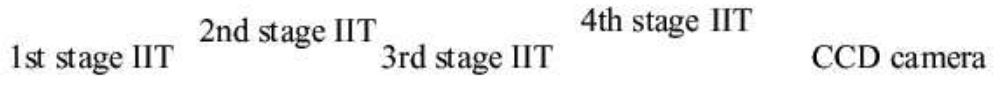

Figure 3.7: The four stages of amplification of the scintillating fiber output [30].

The light from the fibers was amplified using image intensifiers (IIT). Light traveling down the fiber was incident on a photo-cathode where it produced electrons. The electrons were accelerated by an electric field of a few thousand volts to a phosphor screen, where they were focused to reduce image size. When the electrons hit the phosphor screen, they produced photons, which were directed onto another photo-cathode with a quantum efficiency of $20 \%$, and through a fiber optic plane consisting of millions of small diameter fibers. The entire process was repeated four times, leading to an overall amplification of $4 \times 10^{6}$ [27]. The four stages are illustrated in Fig. 3.7. During the third stage of this amplification, the potential was kept lower than normal operating conditions unless a trigger signal was received. The trigger caused the voltage to quickly increase to the nominal voltage. This gated voltage allowed the necessary time for the trigger system to decide if the event was neutrino-like. The last phosphor screen was connected to a charged coupled device, CCD, video camera with 768 x 493 pixels. Each scintillator was mapped onto an area with a 3 pixel diameter. 


\section{The Momentum Measurement System}

The momentum measurement system consisted of a magnet, drift chambers, and the scintillating fibers; these components are shown in Fig. 3.5. To measure the momentum of a track, the track must have passed through the drift chambers. As a charged particle traversed the spectrometer, it traveled through the scintillating fibers, continued through the magnet, and may have traveled through the drift chambers. The incoming and outgoing angles of the charged track were obtained by reconstructing the track from hits in the scintillating fibers and the drift chambers. From this information, the momentum of the charged particle was measured using the thin-lens approximation. The thin lens approximation assumed the particle deflection occurred in the middle of the magnet. The two tracks on either side of the magnet were projected to the center of the magnet, and the angle between the tracks, $\alpha_{x}$ was measured. This angle was used to determine the momentum in the $\mathrm{z}$ direction, $p_{z}$ using

$$
p_{z}=\frac{p_{\text {kick }}}{\sin \alpha_{x}}
$$

where $p_{\text {kick }}$ was measured to be $0.228 \mathrm{GeV}$ [31]. The thin lens approximation was checked by performing a detailed map of the magnetic field and analyzing a sample of tracks with that field map. A comparison with the thin lens approximation agreed within the accuracy of the reconstructed tracks.

\section{The Electromagnetic Calorimeter}

Electron neutrino charged-current events, as well as some tau and charm decays, produced electrons in the final state. These electrons were high energy particles which generated electromagnetic showers as they passed through material. These electrons were identified in three ways [27]. If the electron passed through two or more radiation lengths, the emulsion was used to identify it, as described in Sec. 4.3.3. If the electron was produced in the first half of the emulsion detector, an electromagnetic shower developed in the scintillating fibers. The number of hits and longitudinal cascade of the shower was used to estimate the electron's energy. The final method identified electrons produced in the latter half of the detector using the electromagnetic calorimeter.

The electromagnetic calorimeter (EMCAL), shown in Fig. 3.5, was designed for project E705 [27] and consisted of 400 lead glass and scintillating glass bricks. The lead glass bricks included $25 \%$ lead. The whole apparatus covered an area $4 \mathrm{~m}$ wide and $2 \mathrm{~m}$ tall. The inner region, which consisted of $1007.5 \mathrm{~cm} \times 7.5 \mathrm{~cm}$ scintillating bricks, was used to improve 
position resolution. The middle region consisted of $15 \mathrm{~cm}$ x $15 \mathrm{~cm}$ scintillating blocks; the outer region consisted of $15 \mathrm{~cm} \times 15 \mathrm{~cm}$ lead glass blocks. The configuration is shown in Fig. 3.8. The scintillating blocks were $89 \mathrm{~cm}$ long, corresponding to 20.9 radiation lengths. The lead glass blocks were $41.5 \mathrm{~cm}$ long, or 16.4 radiation lengths. Ten of the calorimeter blocks were calibrated using a test beam at Brookhaven National Laboratory using pions, electrons, and muons which had energies ranging from $1 \mathrm{GeV}$ to $5 \mathrm{GeV}$. The resolution, $\Delta E / E$, was determined to be $10 \%+10 \% / \sqrt{\mathrm{E}}[27]$.

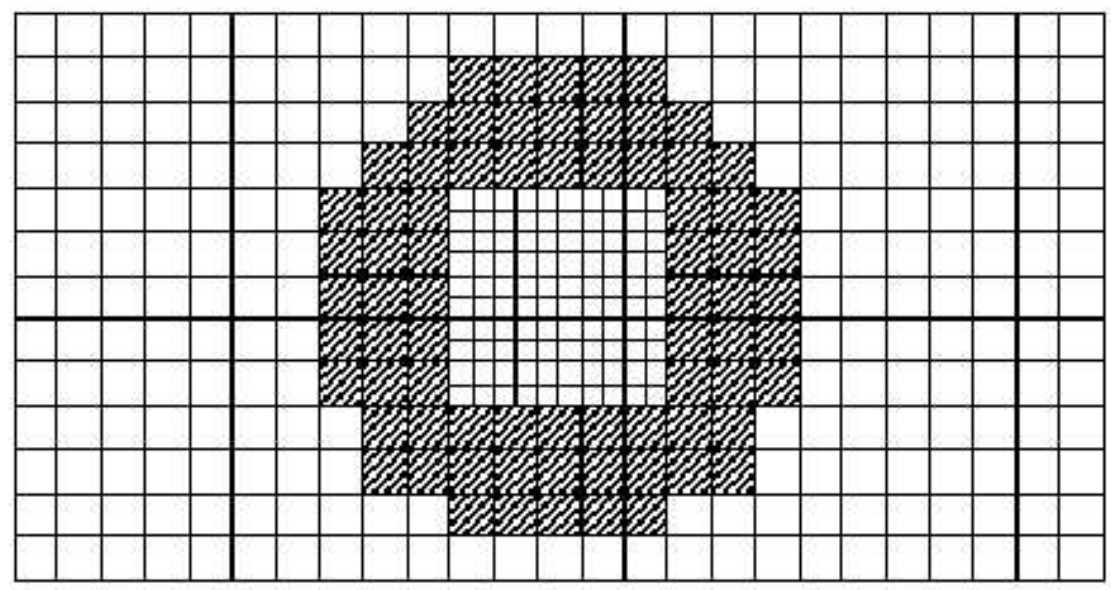

Figure 3.8: The electromagnetic calorimeter [28]. The inner region was constructed of scintillating glass; the outer region was constructed with lead glass.

\section{The Muon Identification System}

If a muon was identified as coming from the neutrino interaction, the event was classified either as a charged-current muon neutrino interaction or a charged-current tau neutrino interaction in which the tau lepton decayed into a muon. Since muons mainly lost energy due to ionization, they passed through more material than electrons or hadrons, which tended to shower. The muon ID system was the farthest downstream component of the spectrometer.

The muon ID system consisted of three H-shaped steel walls interleaved with walls of proportional tubes. The first wall was $6.25 \mathrm{~m} \mathrm{x} 3.7 \mathrm{~m} \mathrm{x} 0.42 \mathrm{~m}$; the other two walls were $5.48 \mathrm{~m} \times 3.25 \mathrm{~m} \times 0.91 \mathrm{~m}$. The walls were H-shaped to avoid the plumes of muons 
discussed in the section on shielding. Planes of proportional tubes were placed on the front and back of the second wall and on the back of the third wall. Some were horizontal and some were vertical, which provided the $\mathrm{X}$ and $\mathrm{Y}$ positions. The muon ID configuration is shown in Fig. 3.5 where the two walls are shown in grey, and the planes of scintillating fibers shown before and after the first grey wall and after the second grey wall. 


\section{Chapter 4}

\section{Data Analysis and Simulation}

A total of $6.6 \times 10^{6}$ triggers occurred in the detectors for $3.6 \times 10^{17}$ protons on target. From calculations, only $10^{3}$ were expected to be neutrino interactions [27]. The neutrinolike interactions were extracted from this data using a series of filters applied to the spectrometer data. Location was attempted for each in the emulsion data. The located neutrino interactions were classified as a charged-current or neutral-current interaction, and the charged-current interactions were further classified by flavor: muon, electron, or tau. The tau candidates were classified using a set of parameters measured in the emulsion and spectrometer. The requirements and parameters used for this analysis were chosen based on results from simulated neutrino events.

\subsection{The Preliminary Data Set}

The DONUT experiment took data over a four month period during the 1996-1997 fixed target run at Fermi National Accelerator Laboratory. Four periods of data were distinguished by different configurations of emulsion modules. Table 4.1 summarizes the configurations of the modules, the total protons on target, and the total number of triggered events for each period.

\subsection{Spectrometer Analysis}

The spectrometer data was used to isolate the events which exhibited neutrino-like characteristics through a series of data filters. The initial filter was incorporated into the 


\section{Characteristics of the Four Run Periods}

\begin{tabular}{|l|c|c|c|c|}
\hline Run Period & 1 & 2 & 3 & 4 \\
\hline Number of protons on dump & $5.4 \times 10^{16}$ & $4.4 \times 10^{16}$ & $1.03 \times 10^{17}$ & $1.55 \times 10^{17}$ \\
\hline Number of triggered events & $1.0 \times 10^{6}$ & $0.8 \times 10^{6}$ & $2.1 \times 10^{6}$ & $2.7 \times 10^{6}$ \\
\hline Station 1 target module & $\mathrm{ECC} 1$ & $\mathrm{ECC} 1$ & $\mathrm{ECC} 1$ & $\mathrm{E} / \mathrm{B} 1$ \\
\hline Station 2 target module & & & $\mathrm{E} / \mathrm{B} 2$ & $\mathrm{E} / \mathrm{B} 2$ \\
\hline Station 3 target module & $\mathrm{ECC} 3$ & $\mathrm{ECC} 3$ & $\mathrm{E} / \mathrm{B} 3$ & $\mathrm{E} / \mathrm{B} 3$ \\
\hline Station 4 target module & - & $\mathrm{E} / \mathrm{B} 4$ & $\mathrm{E} / \mathrm{B} 4$ & $\mathrm{~B} 4$ \\
\hline
\end{tabular}

Table 4.1: Number of protons on target, number of triggered events, and modules used for each period.

spectrometer. This filter, the hardware filter, evaluated each interaction during datataking; if the interaction was deemed neutrino-like, the spectrometer recorded data for this interaction. A software filter reconstructed the interaction and selected events which exhibited certain neutrino-like characteristics. Finally, a visual filter was applied, which involved two physicists classifying each event as a neutrino interaction or a background interaction.

\subsubsection{The Hardware Filter}

The hardware filter, or trigger, was designed to select events which consisted of no charged particles entering the emulsion and one or more exiting the emulsion, as this is the topology of a neutrino interaction. The trigger system consisted of a veto wall and three trigger planes, T1, T2, and T3, interleaved in the scintillating fiber tracker system. The veto wall rejected any events with a charged track entering the emulsion. The rejection rate of the veto wall was $10^{3}: 1$ or better [30], achieved with twenty photo-multipliers combined in an OR logic. The requirement of a charged track leaving the target region was achieved by an adjacency required in the three trigger planes, T1, T2, and T3. An adjacency was defined as a hit in a segment of a trigger plane and a hit in the neighboring segment in the next downstream trigger plane, which ensured tracks were above a minimum angle and below a maximum angle. The minimum angle requirement rejected most penetrating muon tracks. Initially the hardware filter required two or more charged tracks originating from the vertex. In subsequent runs, this requirement was relaxed; only one charged particle was required, but this particle was required to pass through the central region of 
the electromagnetic calorimeter. Approximately $6.1 \times 10^{6}$ events survived the hardware filter and were recorded to tape.

\subsubsection{The Software Filter}

The software filter applied a set of criteria to the recorded events. First the event was reconstructed using pattern-recognition software. This software reconstructed tracks using hits in the scintillating fibers, electromagnetic calorimeter, drift chambers, and muon ID; these tracks were used to predict the location of the interaction, the vertex. An event was labeled as a candidate neutrino interaction if one of the following conditions was met:

- at least one track in the drift chamber pointed back to the most downstream emulsion module

- a vertex was reconstructed using tracks in at least one view of the scintillating fiber tracker system

- a signal of at least $30 \mathrm{GeV}$ was measured in the electromagnetic calorimeter

Many low energy electron and photon scattering events were rejected in this process. The software filter reduced the data set from $6.1 \times 10^{6}$ to approximately 10,000 events.

\subsubsection{The Visual Filter}

The remaining data set consisted mainly of background events caused by muons interacting in the steel which surrounded the detector. Charged particles from these interactions entered from the side and interacted in the detector or produced large showers on the edge of the detector. The visual filter consisted of a team of two physicists evaluating each event. The software reconstruction occasionally added or eliminated hits; in this case, the physicist refitted the event by hand. The reconstructed tracks originating from the neutrino interaction were used to predict the position of the neutrino interaction. The accuracy of the vertex predictions was required to be better than $\pm 0.5 \mathrm{~cm}$ in the transverse directions, $\mathrm{U}$ and $\mathrm{V}$, and $\pm 5.0 \mathrm{~cm}$ in the longitudinal direction, $\mathrm{Z}$, [28]. Virtually all events with five or less tracks had a sufficiently accurate vertex predictions. If the track had a higher multiplicity, it was more difficult to predict an accurate vertex location. Events which contained showers generally could not be uniquely reconstructed; instead, the shower core was used to predict the vertex. The accuracy of each vertex prediction 
was calculated based on the event's topology [27]. A selected neutrino candidate met the following criteria:

- at least one high momentum track or a particle shower pointed to a vertex

- the vertex was within the emulsion target

- the total energy of the event was $>5 \mathrm{GeV}$ as measured by the calorimeter or by the total momentum of charged tracks which passed through the analysis magnet

Four teams of two physicists evaluated each event, then compared results; the discrepancies were reconciled. A total of 1026 events was selected as neutrino interaction candidates. Fig. 4.1 and Fig. 4.2 illustrate the spectrometer data for a interaction. In Fig. 4.1, all components of the spectrometer are shown. The left-most component is the veto wall, which would have been colored if a charged particle had traversed it during the interaction. The next component is the target area, which consisted of the emulsion and scintillating fibers. A close-up of this area is shown in Fig. 4.2. In Fig. 4.2, the vertical lines represent the scintillating fibers, and the blue and pink lines are reconstructed tracks. The hits on these lines indicate a charged particle passed through the scintillating fibers. The size of the green bars represents the pulse height recorded from a scintillating fiber giving a measure of a particle's ionization. The yellow blocks represent the emulsion modules. In these figures, the neutrino interacted in the first module. One of the tracks showers in the scintillating fibers. In Fig. 4.1, the next component is a set of drift chambers which could not be used in the reconstruction due to the large track density through them. Following the drift chambers, there are two boxes drawn, which represent

the analysis magnet. Following the magnet, there are more drift chambers, which were used. The next component is the EMCAL, which shows that some energy was deposited during this event. The last component is the muon ID, which shows no activity in this interaction.

\subsubsection{The Emulsion Volume Filter}

The final data filter applied to the spectrometer data was a fiducial volume filter. If the neutrino interaction occurred on the edges, some tracks left the emulsion volume and could not be reconstructed, prohibiting a decay search. Thus, they were not included in the data set. If an event occurred outside of the "fiducial volume", a volume which included $80 \%$ of the target mass, the event was not included in the data set. Fig. 4.3 and Fig. 4.4 shows an 


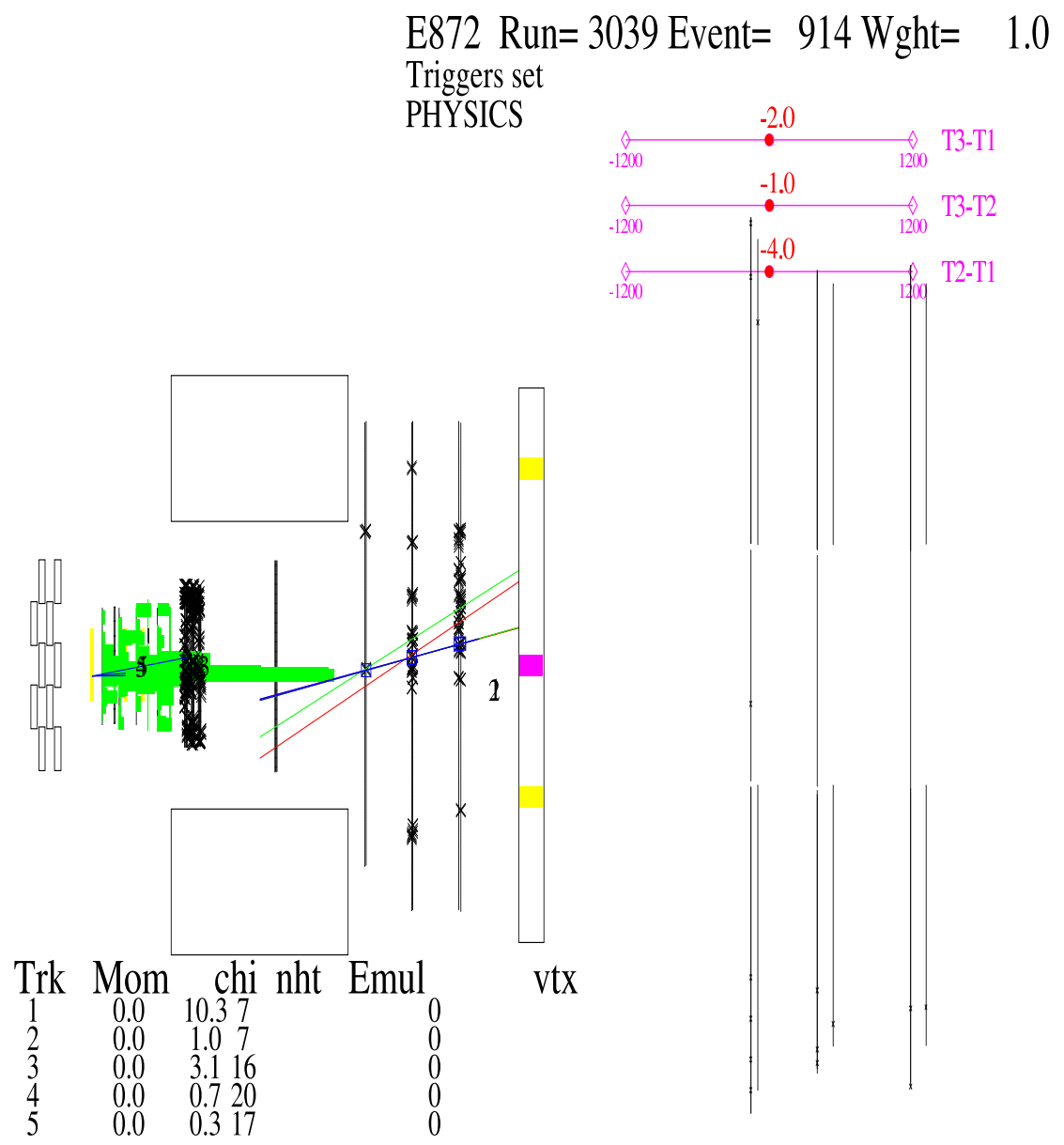

Figure 4.1: Spectrometer data for event 3039_00914. The left-most wall is the veto wall, which is part of the hardware trigger. If a charged particle had passed through, it would be colored. The next component is the target area, where the emulsion and scintillating fibers resided. Activity is present in the scintillating fibers, represented as green rectangles. The darker lines next to the target area are the first set of drift chambers, which were not used because of the high track density. The squares represent the magnet. The next component is a second set of drift chambers, which were used. After the drift chambers is the electromagnetic calorimeter. Energy deposited in the EMCAL is represented by colored squares; three such squares are visible in this event. The final component is the muon ID. If a muon had traversed the walls of the muon ID, hits, represented by black squares, would be visible along the walls. This event had $4.6 \pm 0.7 \mathrm{GeV}$, as measured by the EMCAL. 


\section{E872 Run= 3039 Event $=914$ Wght $=1.0$ U View}
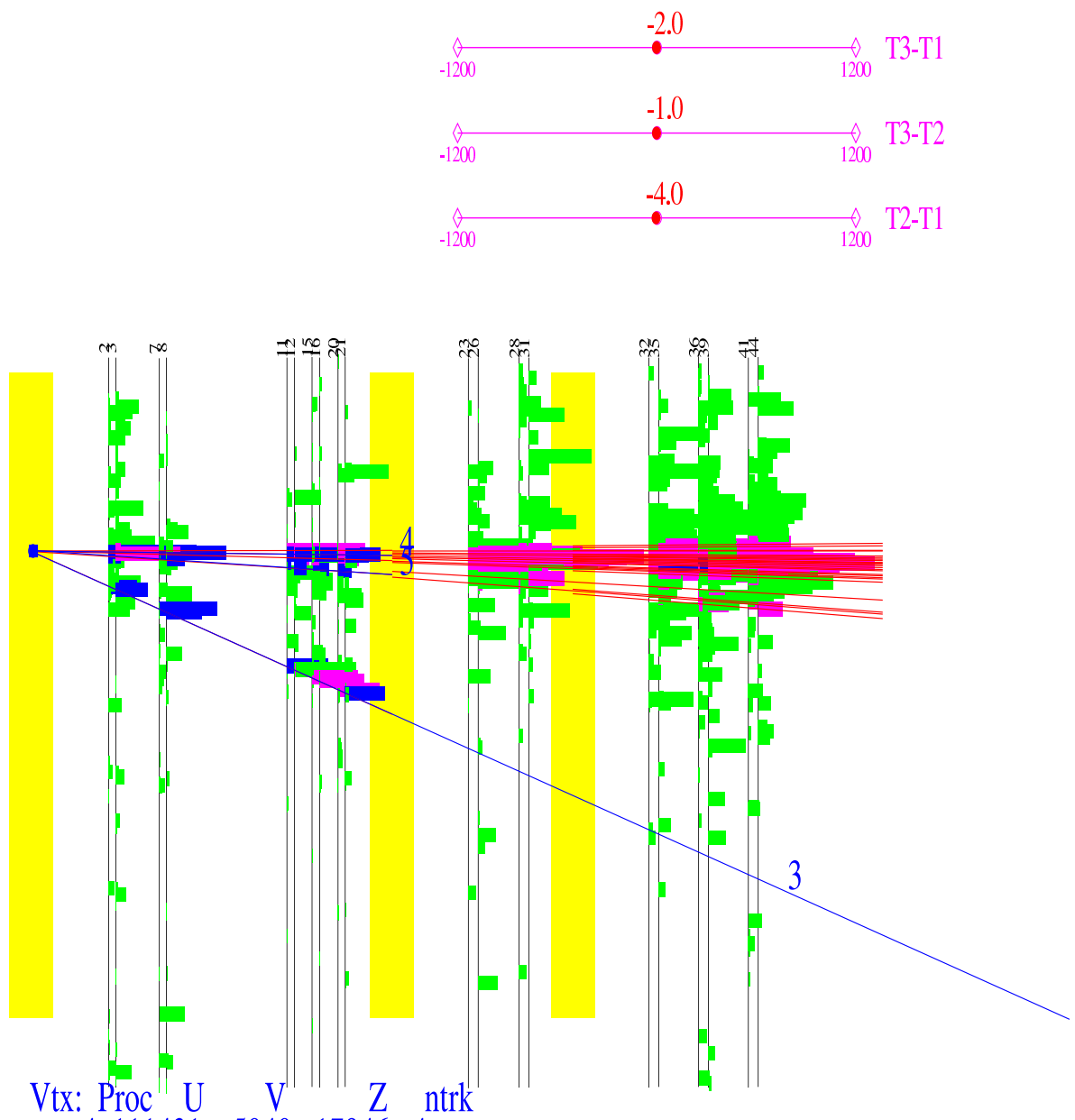

Figure 4.2:

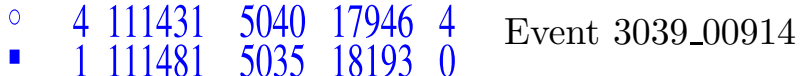

This is the U-view of the scintillating fibers. The light yellow rectangles are the emulsion modules. There were only three exposed during this run, and the neutrino interacted in the first module. The scintillating fibers are the vertical lines. The green hits on the scintillating fiber represent charged particle that traversed the scintillating fibers. The pulse height of these hits varied according to the energy of the particle. The darker scintillating fiber hits are fit to the tracks. A shower developed in the scintillating fibers along track 4. 
event which did not pass the fiducial cut. Fig. 4.3 shows the spectrometer data, and Fig. 4.4 shows a close-up of the target area. As shown in Fig. 4.4, the neutrino interacted on the downstream edge of the emulsion module, which was outside of the fiducial volume. 845 events passed all the filters.

\subsection{Emulsion Data Analysis}

As charged tracks passed through the emulsion, they interacted with the grains in the emulsion and created a record of their trajectory. When the emulsion was developed, these grains appeared darker and were used to reconstruct the particle's path through the emulsion. Each emulsion layer was scanned by an automated digital video camera which recorded every particle track through that layer to a computer file. Computer pattern recognition software linked the particle track segments in each layer to form tracks through the entire scan volume. These tracks were used to locate neutrino interactions, reconstruct their topology, and identify any particles that decayed. The emulsion data also provided a measurement of the momentum of the tracks and identification of electrons.

\subsubsection{Emulsion Scanning}

The automated scanning station consisted of a precision table, a microscope attached to a CCD camera, and pattern recognition software. Each sheet of emulsion was held flat on the table using a vacuum. The microscope-CCD camera apparatus moved across the sheet in steps of $100-150 \mu \mathrm{m}$, and the grain patterns were recorded at each step for sixteen focal planes in the first $100 \mu \mathrm{m}$ of the emulsion sheet. Electronics were programmed to recognize grain positions that were collinear through focal planes. When this pattern was discovered, it was considered a track segment and the angle, position, and pulse height were recorded to a file. The pulse height was the number of focal planes which had aligned darken grains. The data from each sheet of emulsion was combined with other sheets to form a "scan volume" of emulsion. The size of the scan volume depended upon the uncertainty in the vertex prediction, but typically a scan volume was $5 \mathrm{~mm}$ in the transverse directions and $1 \mathrm{~cm}$ in the longitudinal direction, which consisted of approximately $20-25$ sheets of emulsion.

Occasionally part of the emulsion module shifted relative to the rest of the module, which was most likely caused by variations in temperature and applied pressure during the data acquisition [26]. Additional distortions occurred in the modules due to the 


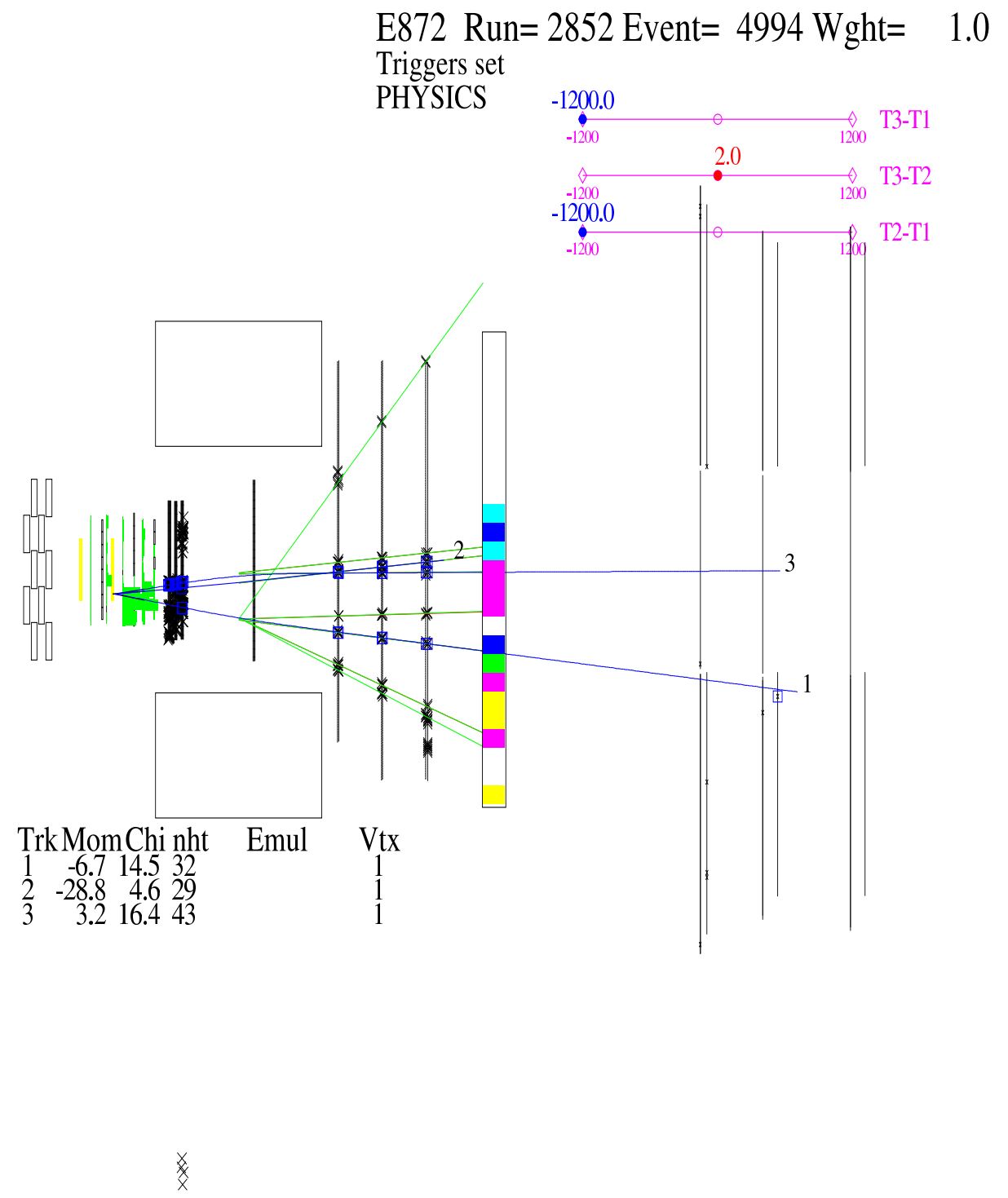

Figure 4.3: Spectrometer data for event 2852_04994. Activity in the scintillating fibers, drift chambers, and the EMCAL is present in this event. $49.2 \pm 5.6 \mathrm{GeV}$ of energy was deposited in the EMCAL. Track 1 has one hit in the muon ID; muons require at least 3 hits in the muon ID. 


\section{E872 Run= 2852 Event= 4994 Wght= 1.0 UView}
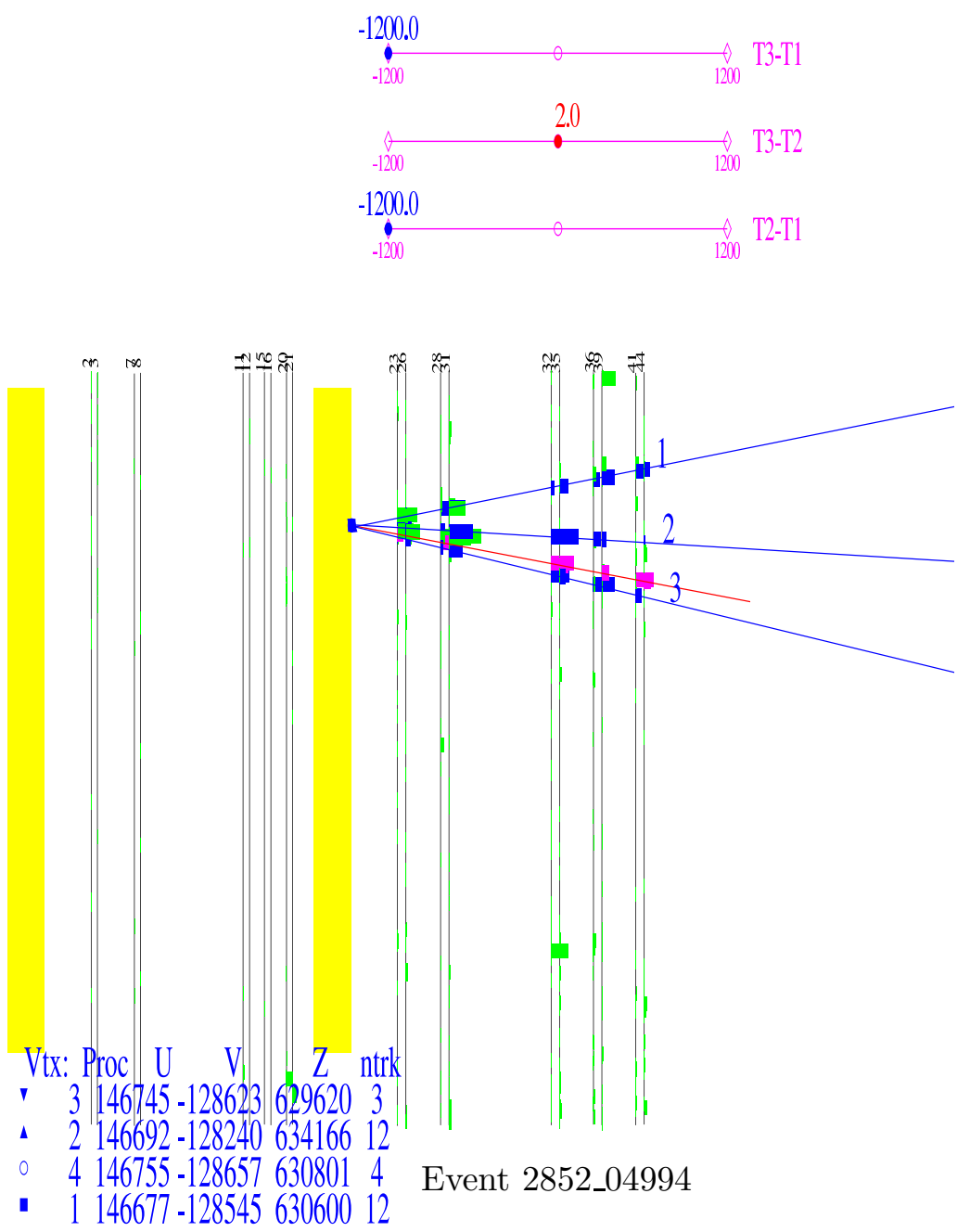

Figure 4.4:

The neutrino interacted on the downstream edge of the second emulsion module. This event was removed from the data set due to the fiducial volume filter. 
accumulation of stress during their production and the developing process. These problems were fixed using alignment with through-going muon tracks.

Global and local alignment corrections were applied to the emulsion data. The global alignment used reconstructed high momentum tracks which traversed the entire scan volume. High momentum tracks were identified by comparing the angles of the track in the upstream and downstream edges of the scan volume. The local alignment correction was applied plate by plate. This correction used the track segments belonging to high momentum tracks. The difference in angle and position between track segments and their associated tracks was measured. If an overall shift in position or an overall rotation was present, the distribution of all deviations showed a peak at this value. If the deviations were random, the distribution was flat. When an overall shift was detected, each segment in the plate was shifted by this deviation. The aligned file was used to reconstruct emulsion tracks.

Tracks were reconstructed by connecting the track segments in each emulsion layer. Each track segment was projected onto the previous plate to identify a track segment matching in angle and position. The process began at the most downstream plate and searched in a cone defined by the RMS scatter. If a matching segment were not found in the immediate upstream plate, the next upstream plate was checked. If there were no segment matching in this plate, the track was considered complete and was written to the file. If a track were simply missing segments in these two or more plates, it was reconstructed as two different tracks, which was corrected later in the analysis. If more than one segment were found that matched in angle and position, the best matched segment was used. The projections were continued until the track stopped or left the emulsion volume. The process of emulsion scanning provided digitized emulsion data with reconstructed tracks, used to locate the position of the neutrino interaction.

\subsubsection{Location in Emulsion Data}

If the neutrino interaction position and tracks were identified in the emulsion data and matched in the spectrometer, the event was considered located. Two methods were used for location: the net-scan method and the scan back method. The net-scan method used a location volume of the emulsion data, while the scan-back method used the changeable sheet emulsion data in addition to the location scan volume.

The net-scan method used a three-dimensional spectrometer track and located all matching emulsion tracks in the scan volume. A three dimensional track was identified 
in the spectrometer using the scintillating fibers, drift chambers, and the electromagnetic calorimeter. A three dimensional track required a reconstructed track in both the Uand V-views; the reconstructed tracks were associated using the $\mathrm{X}$-view, an independent view. All emulsion tracks in the location scan volume matching in position and angle were located. Once matching emulsion tracks were found, each track was followed upstream until it ended. If other tracks also originated at this point, it was a vertex candidate. Each vertex was analyzed using a ROOT [32] three dimensional display. If the vertex appeared real, the emulsion tracks were projected onto the spectrometer data. If these emulsion tracks were, indeed, produced by a neutrino interaction, they contained hits in other sensitive parts of the detector. If the emulsion track matched hits in the scintillating fibers, drift chambers, electromagnetic calorimeter, or the muon ID, the vertex was assumed to be the true vertex; its position and track information were sent to Nagoya University, where the vertex was verified manually by looking at the emulsion under a microscope.

The scan-back method was similar to the net-scan method, except it relied on changeable sheets placed on the front and back of the emulsion modules which were changed weekly. In this method a track, identified in the spectrometer data as described above, was projected onto the changeable sheet. Due to a lower track density in the changeable sheets, typically few matching emulsion track segments existed. Each matching track segment was projected upstream to the location scan volume, and other track segments which matched the projection were used to form a track. The track was followed upstream until its origin. This position was searched for other tracks which originated at this point. If the events contained a shower, this method used the core of the shower to locate the vertex. A total of 539 events were located using these two methods.

Fig. 4.5 shows an example of the emulsion data. The darker marks represents the emulsion track segments. The line was drawn to connect the segments. Fig. 4.6 shows the same vertex from a different perspective, but includes all the background emulsion data. The emulsion is made of ECC 800 sheets, which is a $800 \mu \mathrm{m}$ layer of plastic with a $100 \mu \mathrm{m}$ emulsion on either side combined with a $1 \mathrm{~mm}$ layer of steel, as evident in Fig. 4.6. The collection of emulsion segments show the $100 \mu \mathrm{m}$ layers of emulsion. The white spaces between are alternating layers of plastic and steel.

\subsubsection{Additional Emulsion Information}

When an event was located in the emulsion, another emulsion volume, the decay volume, was created. This volume began two emulsion plates upstream of the vertex, was centered 


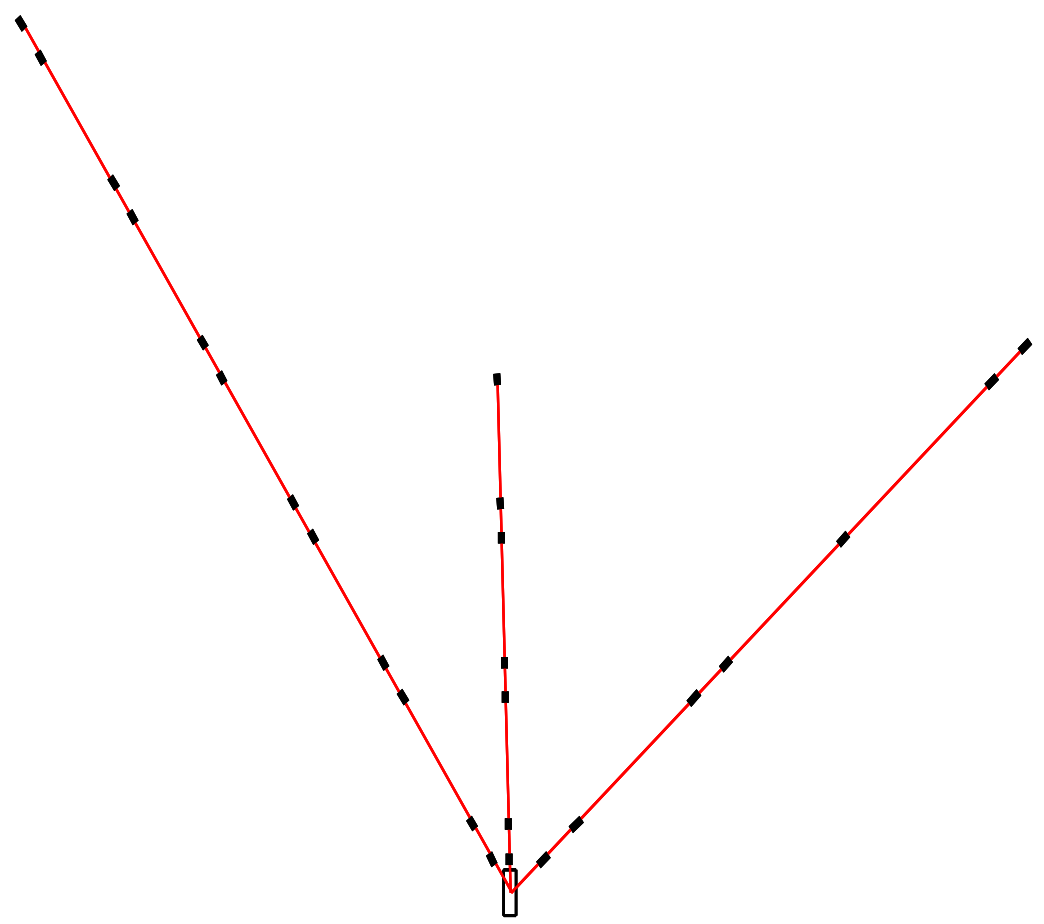

Figure 4.5: The emulsion data for neutrino event. The dark segments are the emulsion track segments, each $100 \mu \mathrm{m}$ in length. The lines are drawn to connect the segments. 


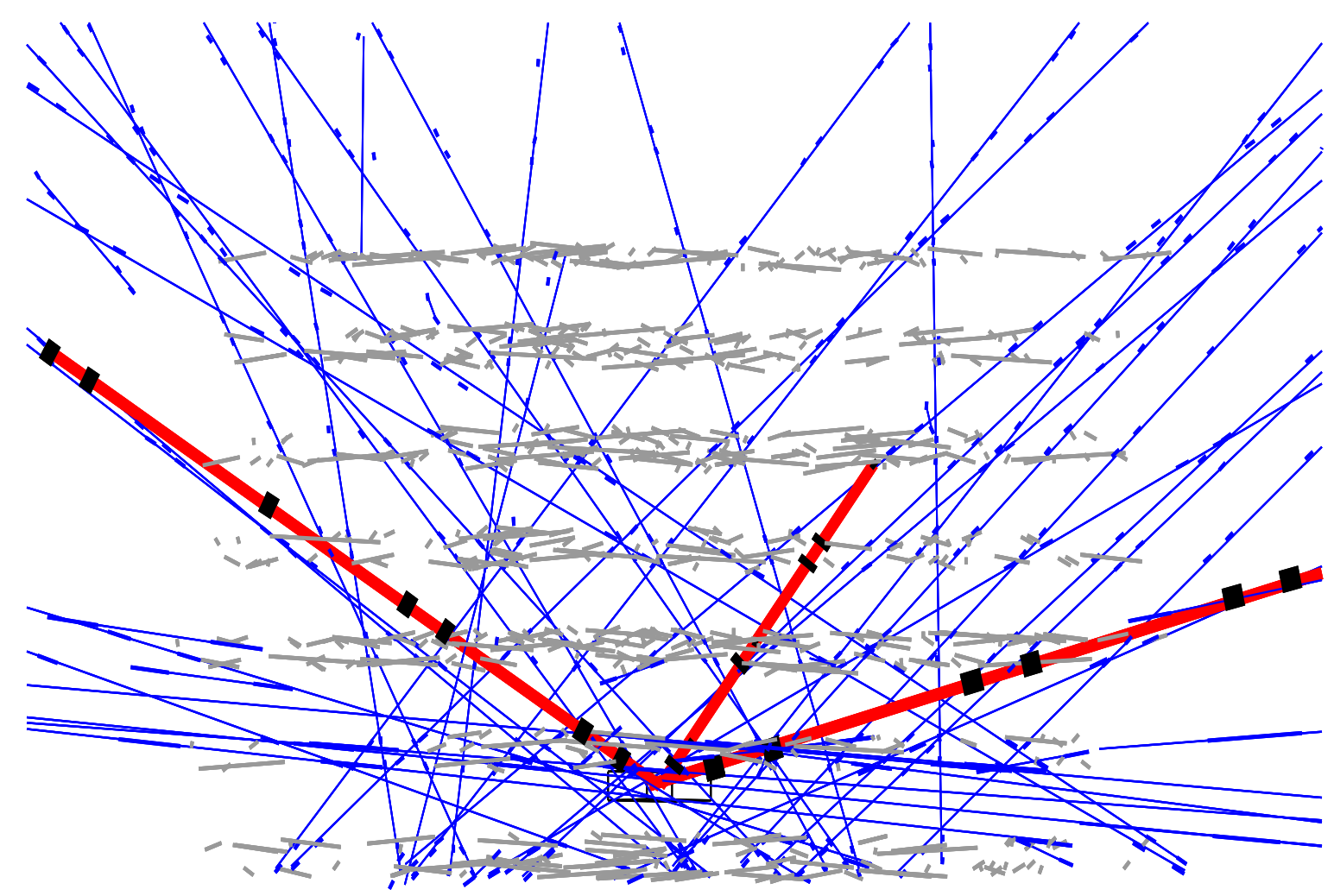

Figure 4.6: The emulsion data for the same neutrino event including all background data. This is a slightly different perspective than in Fig. 4.5. The horizontal layers are made of emulsion track segments. Some are connected to tracks, but most are not. The lighter lines are tracks formed by connecting the emulsion segments. The darker tracks are the tracks in Fig. 4.5. 
around the vertex, and was generally smaller than the location scan volume, $2.6 \times 2.6$ by $10 \mathrm{~mm}^{3}$. Additionally each primary track from the interaction had an associated emulsion volume, which included all emulsion data along the track. These volumes were used for decay searches, particle identification, and the measurement of momentum using multiple scattering.

\section{Locating Kinks and Tridents in Emulsion}

The tau lepton produced in a charged-current tau neutrino interaction decayed to either one charged particle, a kink event, or three charged particles, a trident event. A kink had to satisfy two conditions: the daughter track must have a distance of closest approach to the primary vertex of $5 \mu \mathrm{m}$ or less, and the angle between the tracks must be at least 10 mrad. Two methods were used to search for kinks. The first assumes the kink angle was

small, less than $10 \mathrm{mrad}$, and the parent and daughter tracks were reconstructed as a single track. In this case, each emulsion track was broken into two tracks at each track segment. The tracks were fit separately. If the fit improved after separation, the two sections were reclassified as two tracks. If the angle between these two tracks was greater than $10 \mathrm{mrad}$, it was classified as a kink candidate. If the angle between the daughter and the parent tracks was greater than $\sim 15 \mathrm{mrad}$, the tracks were reconstructed separately. If a primary track stopped in the emulsion volume, the next downstream plate was checked for tracks which began where the parent track ended. If the intersection met the requirements listed above, the events was classified as a candidate kink event. The downstream plate of the stopping track was also searched for a trident vertex. If three tracks originated at the end of a primary track, the event was considered a candidate trident event.

\section{Emulsion Momentum Measurement}

All emulsion tracks from each neutrino interaction were momentum analyzed using multiple scattering due to the Coulomb interaction. As the particles traveled through the emulsion, they were deflected by small amounts. The root mean square of the position displacement, $y$, is a function of momentum, $p$, a scattering constant, $k$, the speed of the particle, $\beta$, the thickness of the material, $t$, and the radiation length of the material, $X_{0}$, and is $[25]$

$$
\sqrt{\left\langle y^{2}\right\rangle}=\sqrt{\frac{2}{3}} \times \frac{k}{p \beta} \times \sqrt{\frac{t}{X_{0}}} .
$$




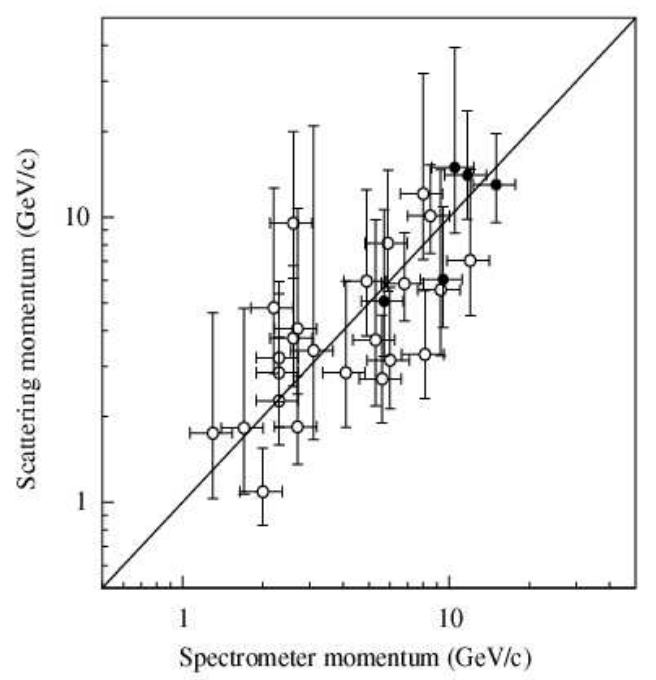

Figure 4.7: Comparison of the spectrometer and emulsion momentum measurements. This data excludes tracks associated with showering events and any tracks which did not have a clear fit in the drift chambers or EMCAL.

For a $1 \mathrm{GeV} / \mathrm{c}$ particle in the ECC emulsion, the expected deflection was $4.0 \mu \mathrm{m}$ due to the steel sheet between the two emulsion layers. The position displacement of a track was measured, and the momentum was calculated using Eq. 4.1. The details of the measurement were described in Ref. [26]. $\frac{d E}{d x}$ corrections were considered and were negligible. The emulsion measurements were checked using those tracks which were also measured in the spectrometer. The results, shown in Fig. 4.7, showed reasonable agreement. The emulsion momentum measurement was used when no spectrometer measurement could be made or when the particle's energy was so low that it lost a significant amount of its energy traversing the material in the upstream end of the spectrometer. The spectrometer momentum measurements were more accurate for high momentum tracks, but only available for a subset of the tracks. A spectrometer momentum measurement required the track to be unambiguously linked through the drift chambers. 


\section{Electron Identification}

Electrons were identified through the observation of electromagnetic showers. In the spectrometer these showers were visible in the scintillating fiber tracker and in the pattern of energy deposited in the calorimeter. In the emulsion, the electron lost energy through bremsstrahlung. The photon emitted during the bremsstrahlung process produced an electron-positron pair, which was visible as a "vee" pointing back to the electron track. If these vee's were observed along a track in the emulsion, the track was classified as an electron.

\subsection{Event Simulation}

The cross section measurements required several quantities that could not be measured directly from the data, such as the efficiencies, the number and energy spectra of the produced neutrinos, and the target acceptance. These quantities were calculated using the Monte Carlo, which included an event generator, simulated leptonic and hadronic interactions, and a detector simulation. The different parts of the Monte Carlo are described in this section.

The generation of the tau neutrino energy spectrum required input from the production of the $D_{s}$ from $800 \mathrm{GeV}$ proton-nucleon interactions and the decay of the tau leptons produced from the decay of the $D_{s}$. Since the $D_{s}$ cross section has never been measured in $800 \mathrm{GeV}$ proton-nucleon interactions, it was assumed that the differential cross section shape for $D_{s}$ production is equal to the charged and neutral $D$ production, which has been measured to be consistent with

$$
\frac{d^{2} \sigma}{d x d p_{T}^{2}}=e^{-b p_{T}^{2}}(1-|x|)^{n},
$$

where $x$ is the longitudinal momentum fraction,

$$
x=\frac{2 p_{z}}{\sqrt{s}}
$$

and

$$
p_{T}=\sqrt{p_{x}^{2}+p_{y}^{2}}
$$

Experimental measurements of the $n$ and $b$ terms for $800 \mathrm{GeV}$ proton-nucleon interactions

are shown in Table 4.2. The uncertainties in these measurements gives an uncertainty in the cross section measurement, which is discussed in Sec. 6.3.2. 


\begin{tabular}{|c|c|c|}
\multicolumn{1}{c}{$b$ and $n$ for $800 \mathbf{~ G e V}$ protons } \\
\hline Experiment & $\mathrm{b}$ & $\mathrm{n}$ \\
\hline E $653[33]$ & $0.84_{-0.08}^{+0.10}$ & $6.9_{-1.8}^{+1.9}$ \\
\hline E743[34] & $0.8_{-0.2}^{+0.2}$ & $8.6_{-2.0}^{+2.0}$ \\
\hline Average & $0.83_{-0.11}^{+0.11}$ & $7.7_{-1.4}^{+1.4}$ \\
\hline
\end{tabular}

Table 4.2: The averages of the two measurement were used for $b$ and $n$ in the Monte Carlo. The values were varied using a Gaussian distribution with a width equal to the uncertainties in the measurements.

The decay of the $D_{s}$ was assumed to occur at the center of the tungsten beam dump in the transverse direction and at a position L from the front of the beam dump. L was randomly taken from a distribution proportional to $e^{-L / \lambda}$, where $\lambda$ is the nuclear interaction length of the tungsten, $9.65 \mathrm{~cm}$. Both $n$ and $b$ were varied using a Gaussian distribution centered at the mean with a width equal to the uncertainties in the measurements. The $D_{s}$ particles were projected to a decay position, calculated from a randomly chosen lifetime for the $D_{s}$. The $D_{s}$ was decayed isotropically to a tau lepton and tau neutrino. Each event was assigned a weight which reflected the phase space availability; this was done using a CERNLIB routine called GENBOD [35]. The tau decay was simulated in a similar manner, projected to a decay position and decayed. Additional charm decays were required to calculate the electron and muon neutrino fluxes. These decays were simulated using the same techniques as described above.

Since muon neutrinos from light meson decays composed approximately half of the muon neutrino sample, these decays were also simulated. A GEISHA [36] simulation was used to create light mesons from the interaction of $800 \mathrm{GeV}$ protons and tungsten. A weight was assigned to each pion and kaon equal to the probability of it decaying before re-interacting in the beam dump. The decay length, $L$, was calculated using a lifetime chosen from a distribution proportional to $e^{-t}$, where $t$ is the lifetime. The interaction distance was taken from a distribution proportional to $e^{-L / \lambda}$. The semi-leptonic channels were considered.

The interactions between the neutrinos and the target nuclei in the emulsion module were simulated using the LEPTO program package [37]. Five specific event types were simulated: the charged-current tau neutrino interaction, the charged-current electron neutrino interaction, the prompt charged-current muon neutrino interaction, the non-prompt 
muon neutrino interaction, and the neutral-current interaction. Particles produced in the simulated neutrino interactions were propagated through the spectrometer using the GEANT program package [38]. The generated events had an associated weight that corresponded to the product of the probability of neutrino generation and interaction. Each of the four emulsion target configurations were represented in the simulation; these representations included the steel, emulsion, and plastic components of the emulsion modules. The components of the spectrometer were represented as separate sensitive elements in the GEANT simulation. The forty-four planes of scintillating fibers were represented as separate sensitive elements. The hit positions in the scintillating fibers were smeared by a Gaussian distribution with a width of $0.1 \mathrm{~mm}$, and the hit was generated in the fiber closest to the smeared position. The drift chambers were also sensitive elements in the simulation. The electromagnetic calorimeter was a separate sensitive element in the GEANT simulation. The total energy in each block was found by adding up the contributions from all particles passing through it.

A set of muon neutrino charged-current interactions were used to calibrate the Monte Carlo because they were easily selected from the data. The simulated detector hits were smeared to match the distribution found in the data. The position uncertainties and efficiencies of the simulated detector elements were adjusted to produce the resolutions in the data. The particle production thresholds in LEPTO [37] and the tracking thresholds in GEANT [38] were adjusted using the observed particle multiplicity, track angles, calorimeter energy, and other event parameters.

Two different methods were used to check the results of the Monte Carlo. The first involved calculating the number of neutrino-nucleon interactions in the data. The second involved comparing the simulated interacted energy spectrum for muon and electron neutrinos to experimentally measured energy spectra. The number of events in the data set is consistent with the number of events calculated using the Monte Carlo within statistical uncertainty [30]. A second check of the number of produced neutrinos used events with more than $20 \mathrm{GeV}$ in the EMCAL and more that $5 \mathrm{GeV}$ in the central EMCAL. The number of predicted events of this type matched the measured number of events within statistical uncertainty. The second check used the energy spectrum for electron and muon neutrino interaction from E613 [39]. This spectrum was consistent with the simulated spectrum [40]. 


\subsection{Parameters for Statistical Analysis}

A statistical analysis, described in Ch 5, was performed on all tau and charm candidates. The parameters used to identify the event in this analysis were the production angle, $\theta$, the polar angle asymmetry, $\Delta \phi$, the decay length of the parent track, $L$, the kink angle, $\alpha$, the daughter momentum, $P_{d}$, and the sum of the daughter impact parameters, $\Sigma I P$. The Monte Carlo was used to simulate these parameters for tau neutrino interactions and muon and electron neutrino interactions which resulted in either a charm which decayed to one or three charged particles or a non-charm hadron which interacted and produced one or three charged particles. The charm and interaction events were the main potential backgrounds to the signal events. The analysis provided relative probabilities for each of these possibilities for each tau and charm candidate. Each parameters and its simulated distribution is described below. Fig. 4.8, Fig. 4.9, and Fig. 4.10 illustrate each parameter. The simulated distributions were normalized using the weights provided for each event in the Monte Carlo; these weights were a product of the probability of generation and interaction

The production angle $\theta$ is the angle between the neutrino direction and the direction of the parent,

$$
\theta=\cos ^{-1}\left(\hat{P}_{\mathrm{p}} \cdot \hat{P}_{\nu}\right)
$$

where $\hat{P}_{\mathrm{p}}$ is a unit vector in the direction of the parent track, and $\hat{P}_{\nu}$ is a unit vector in the direction of the neutrino. The neutrino direction was calculated from the center of the beam dump to the interaction point; this assumes the neutrino was produced in the center of the beam dump. The maximum uncertainty for this assumption was 1.5 mrad, which occurred when the neutrino was produced at the outer edge of the beam dump. Since the tracks undergo multiple scattering, the direction of the parent track was measured from the first three emulsion segments on the emulsion track. This parameter provided significant distinction between the tau and interaction events. The production of tau leptons in high energy tau neutrino interactions was mainly in the forward direction; therefore, the tau distribution is peaked near zero. The distributions of $\theta$ for simulated tau, charm, and interaction events are shown is Fig. 4.11 [29].

$\Delta \Phi$ is is the polar angle asymmetry, which is calculated using Eq. 4.6.

$$
\Delta \Phi=\tan ^{-1}\left(\frac{\sum_{i \neq \tau} \sin \Phi_{i}}{\sum_{i \neq \tau} \cos \Phi_{i}}\right)-\Phi_{\text {parent }},
$$




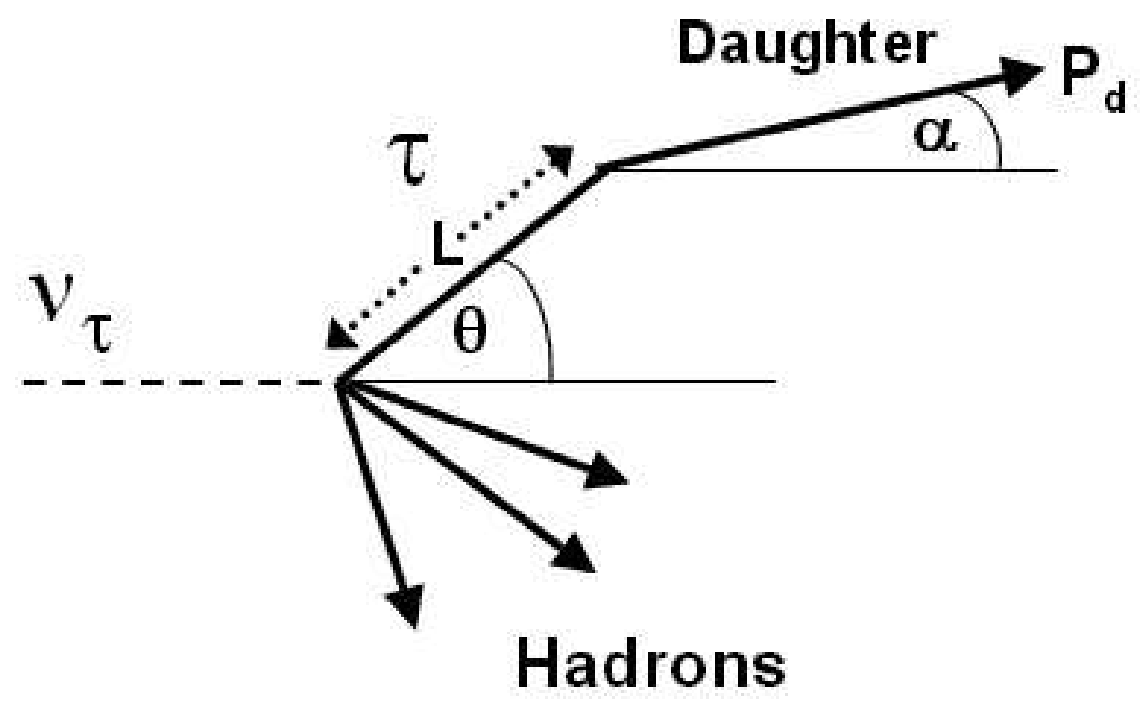

Figure 4.8: This figure illustrates four of the five parameters used in the kink analysis. The production angle, $\theta$, is the angle between the neutrino direction and the direction of the parent, which is calculated using Eq. 4.5. The decay length, $L$, is defined as the distance from the neutrino interaction position to the point of the decay or interaction, which was measured in the emulsion data. The daughter angle, $\alpha$, is the angle between the direction of the parent track and the direction of the daughter. The daughter momentum, $P_{\mathrm{d}}$, was measured through multiple scattering in the emulsion data or in the spectrometer. 


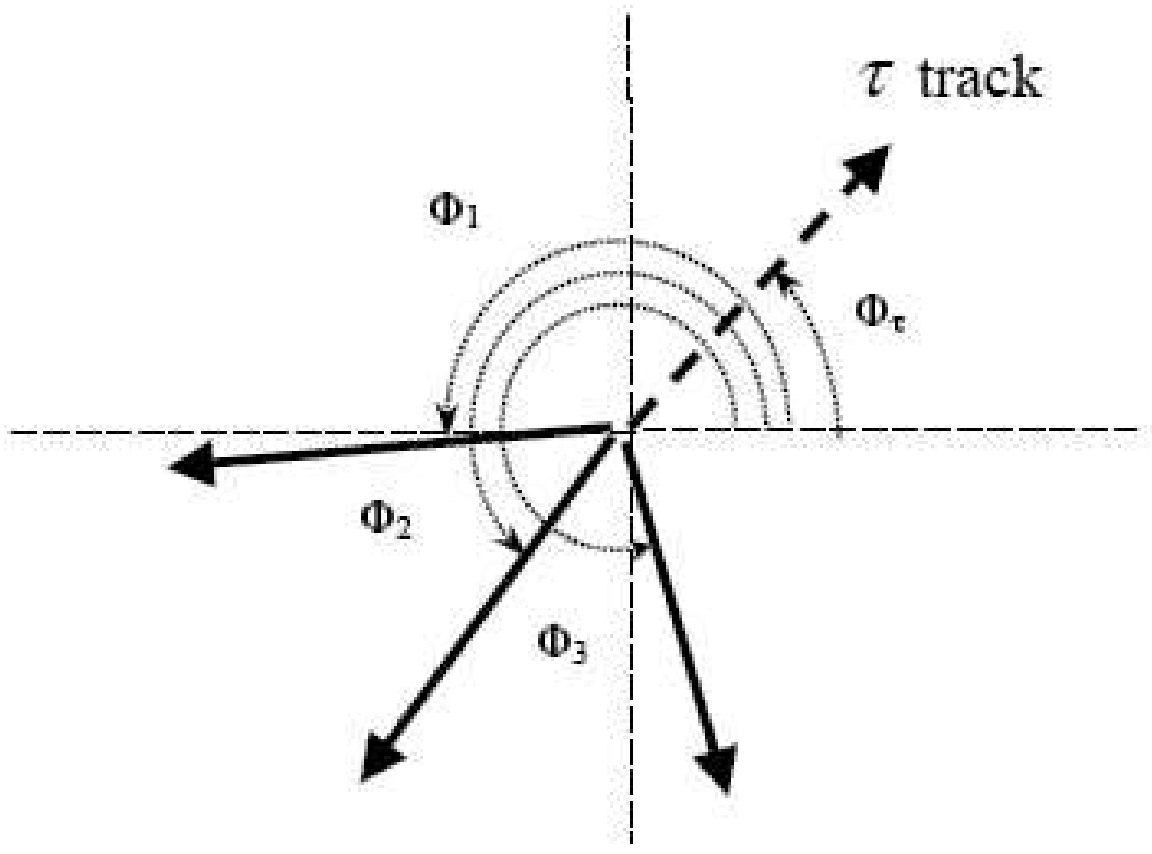

Figure 4.9: This figure illustrates the polar angle, $\Delta \phi$. The figure shows the event tracks projected into a plane perpendicular to the beam direction. $\Delta \Phi$ is calculated using Eq. 4.6. The polar angle is a measure of the transverse momentum balance; therefore, the tau distribution is peaked at $\pi$. Background events would have a random distribution of tracks in this projection and, thus, a flat distribution. 


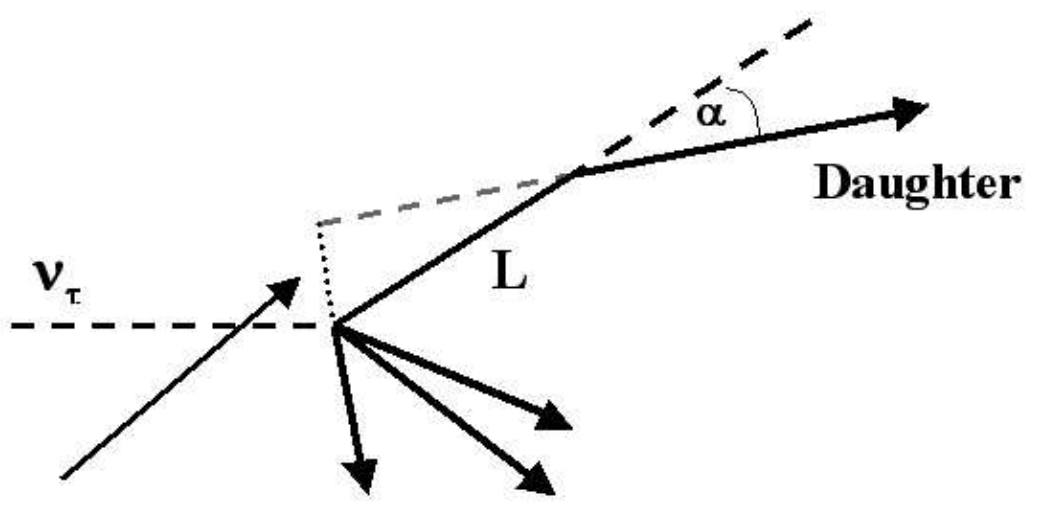

\section{Impact Parameter} $\mathbf{I P}=\mathbf{L} \sin \alpha$

\section{Hadrons}

Figure 4.10: The trident parameters. The production angle and decay length are the same for the kink and trident events. The additional parameter is $\Sigma I P$, the sum of the impact parameters of the daughter particles, which was used only for the trident events. The impact parameter is calculated using the daughter angle and the length of the parent track, as shown in Eq. 4.9. 


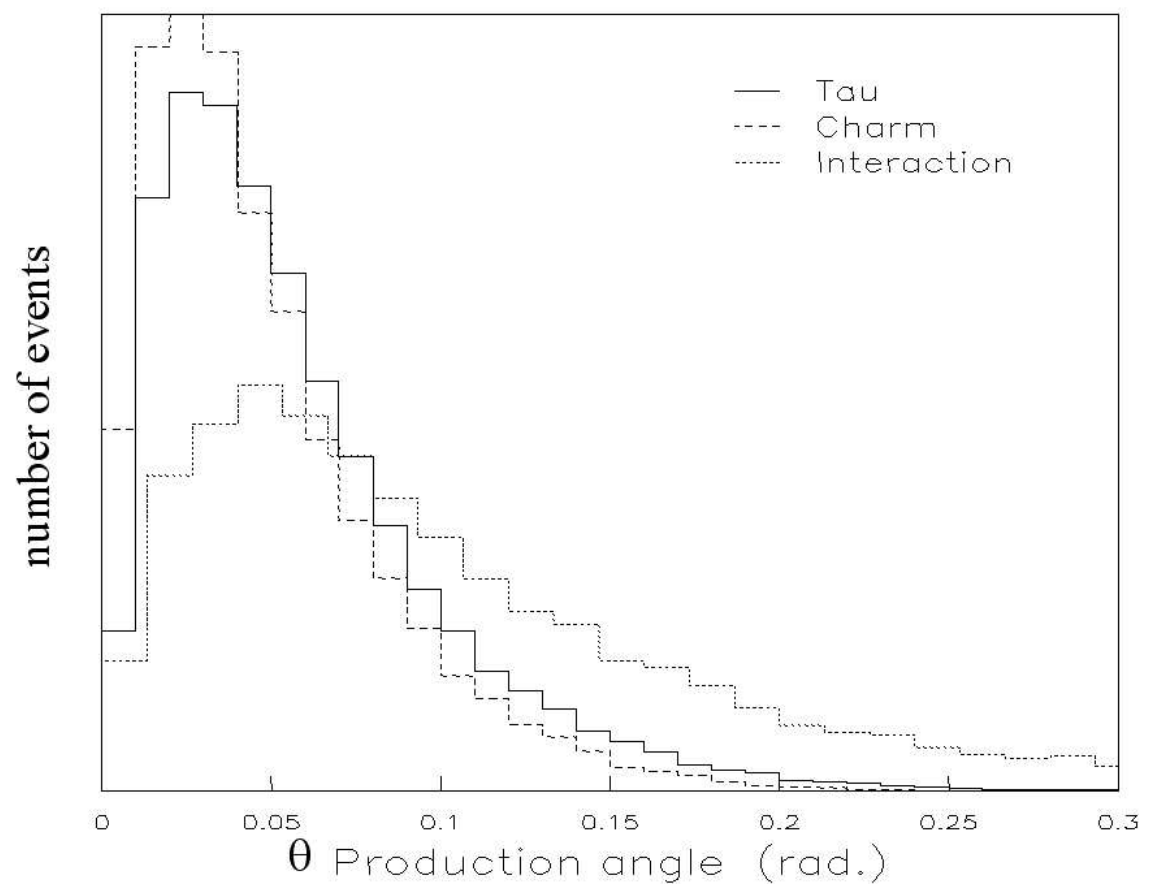

Figure 4.11: Distributions of $\theta$ for simulated tau, charm, and interaction events. This parameter provided significant distinction between the tau and interaction events. The production of tau leptons in high energy tau neutrino interactions was mainly in the forward direction; therefore, the tau distribution is peaked near zero. The distributions were normalized using the Monte Carlo weight of each event. The weight is the product of the probability of production and interaction. 
where

$$
\Phi_{i}=\tan ^{-1}\left(\frac{\tan \alpha_{y}}{\tan \alpha_{x}}\right) \text { for } \alpha_{y} \geq 0
$$

and

$$
\Phi_{i}=\tan ^{-1}\left(\frac{\tan \alpha_{y}}{\tan \alpha_{x}}\right)+\pi \text { for } \alpha_{y}<0 .
$$

where $\alpha_{y}$ and $\alpha_{x}$ are the production angles of the parent track in the Y-Z and X-Z planes. Using this definition of $\Delta \phi$, the tau distribution is peaked at $\pi$. The polar angle imbalance, $\Delta \Phi$, is the measure of the momentum balance between the parent and all other particles produced in the interaction. The particles which produced background kinks were not constrained to this momentum balance since the kinks were not caused by the lepton from the neutrino interaction. The polar angle provided significant distinction between the charm and interaction backgrounds and the tau events. The $\Delta \Phi$ distributions for simulated tau, charm, and interaction events are shown in Fig. 4.12 [29].

The decay length, $L$, is the length of the parent particle, defined as the distance from the neutrino interaction position to the point of the decay or interaction, as measured in the emulsion data. This parameter provided distinction between the interaction backgrounds and the tau events. The tau and charm distributions are similar; they are exponentials and are strongly peaked at zero. The decay length distributions are shown for simulated tau, charm, and interaction events in Fig. 4.13.

The daughter angle, $\alpha$, is the angle between the direction of the parent track and the direction of the daughter. This was calculated in a manner similar to the production angle, using the last three segments of the parent track and the first three segments of the daughter track. This parameter was only used for the kink events. The kink angle provided significant distinction between the tau and interaction events. Large kinks were more probable for interaction events than for tau events because there were many more low momentum hadrons. The distributions of this parameter for simulated tau, charm, and interaction events are shown in Fig. 4.14 [29].

The daughter momentum, $P_{\mathrm{d}}$, was measured either using the deflection of the track in the magnet or using the emulsion data to measure the momentum from multiple scattering. This parameter was only used for the kink events. The uncertainty in the multiple scattering measurement of momentum was due to a limited number of track segments in the emulsion. The tracks with low momentum scattered more; thus, the momentum measurement from multiple scattering was better for lower energy tracks. The lower momentum tracks often did not reach the drift chambers, and, therefore, had no spectrometer 


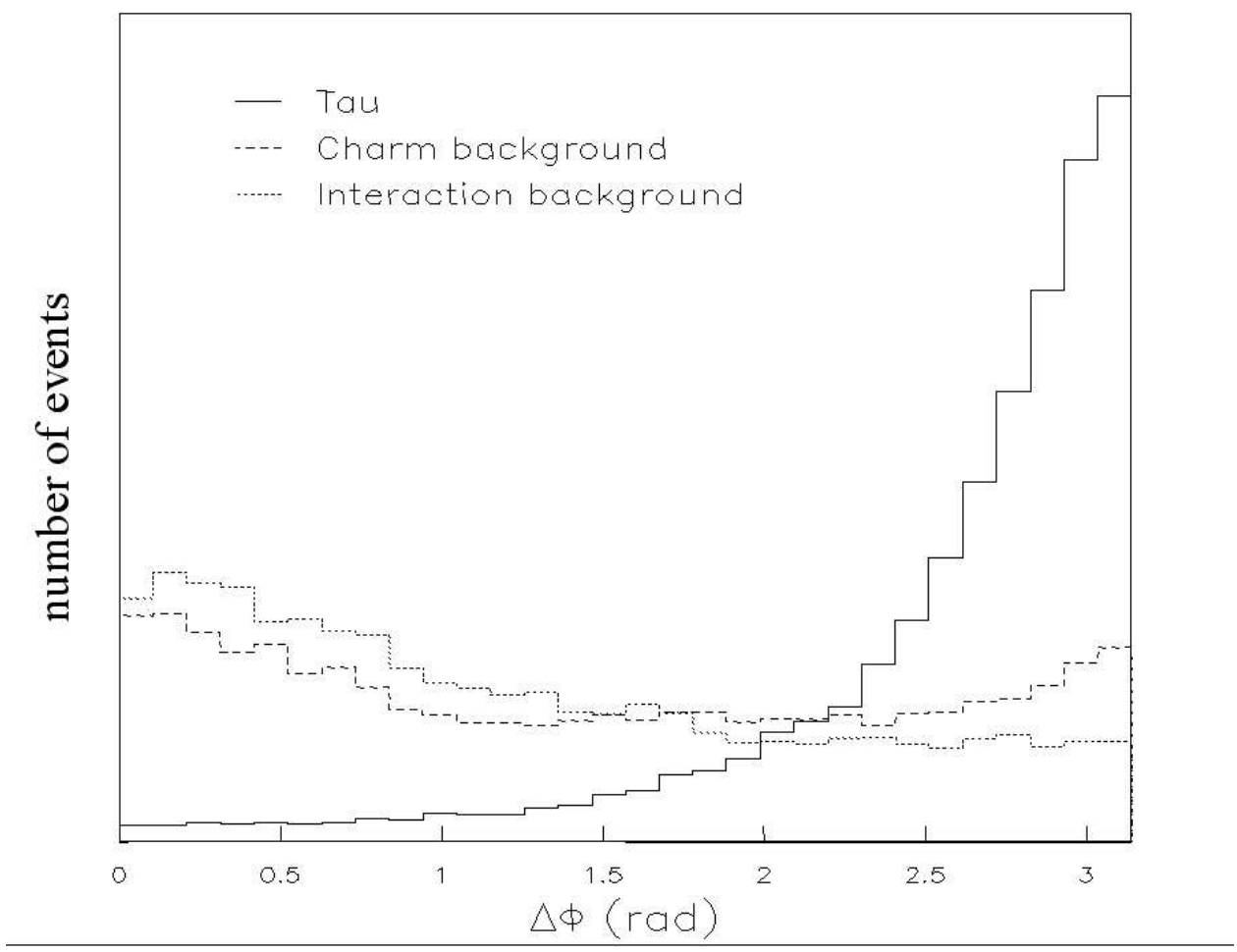

Figure 4.12: Distributions of $\Delta \Phi$ for simulated tau, charm, and interaction events. The polar angle is a measure of the transverse momentum balance. The particles which produced background kinks were not constrained to this momentum balance since the kinks were not caused by the lepton from the neutrino interaction. The polar angle provided significant distinction between the charm and interaction background and the tau events. 


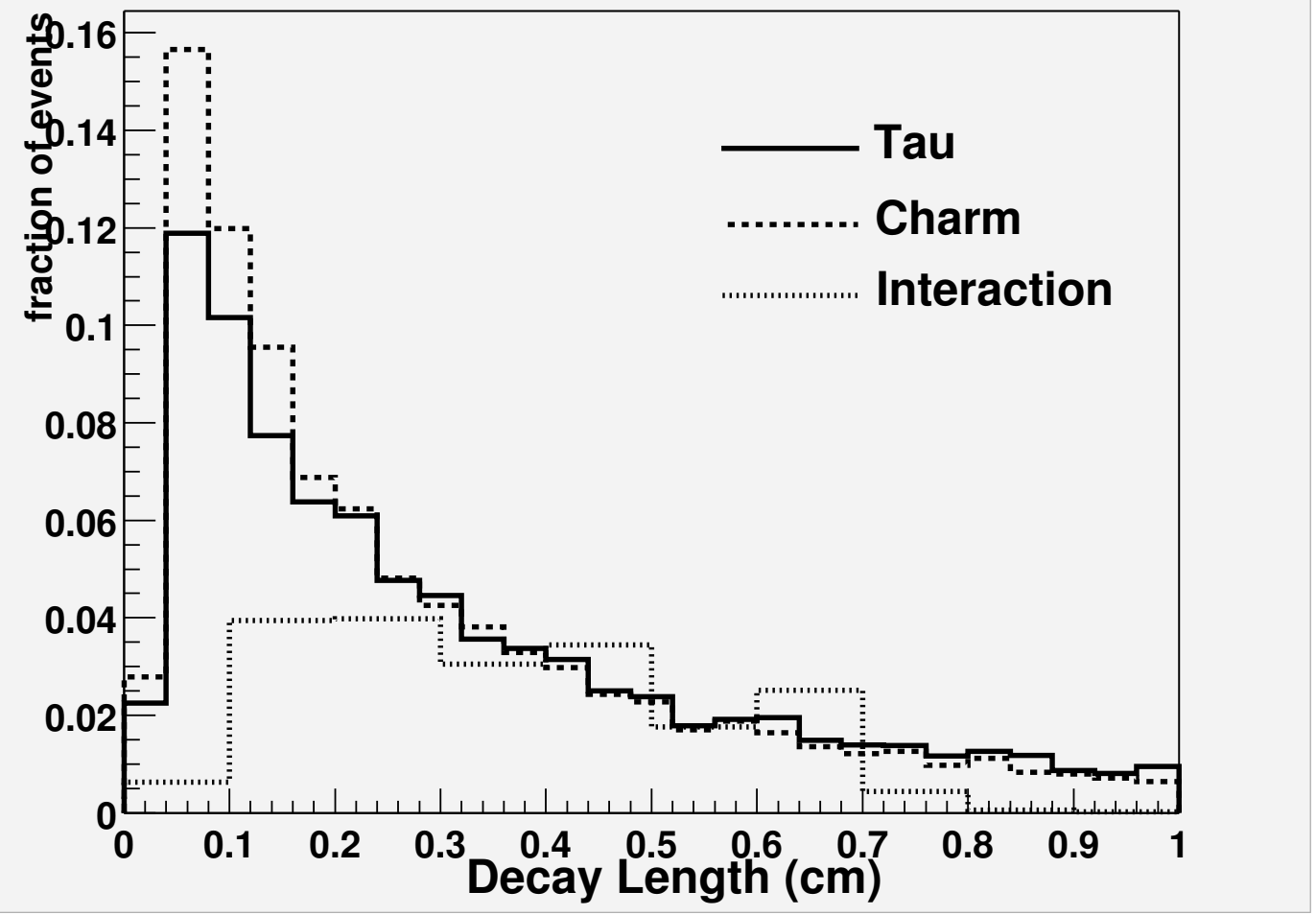

Figure 4.13: Distributions of $L$ for simulated tau, charm, and interaction events. $L$, was defined as the distance from the neutrino interaction position and the point of the decay or interaction, which was measured in the emulsion data. This parameter provided distinction between the interaction backgrounds and the tau events. The tau and charm distributions are similar; they are exponentials and are strongly peaked at zero. 


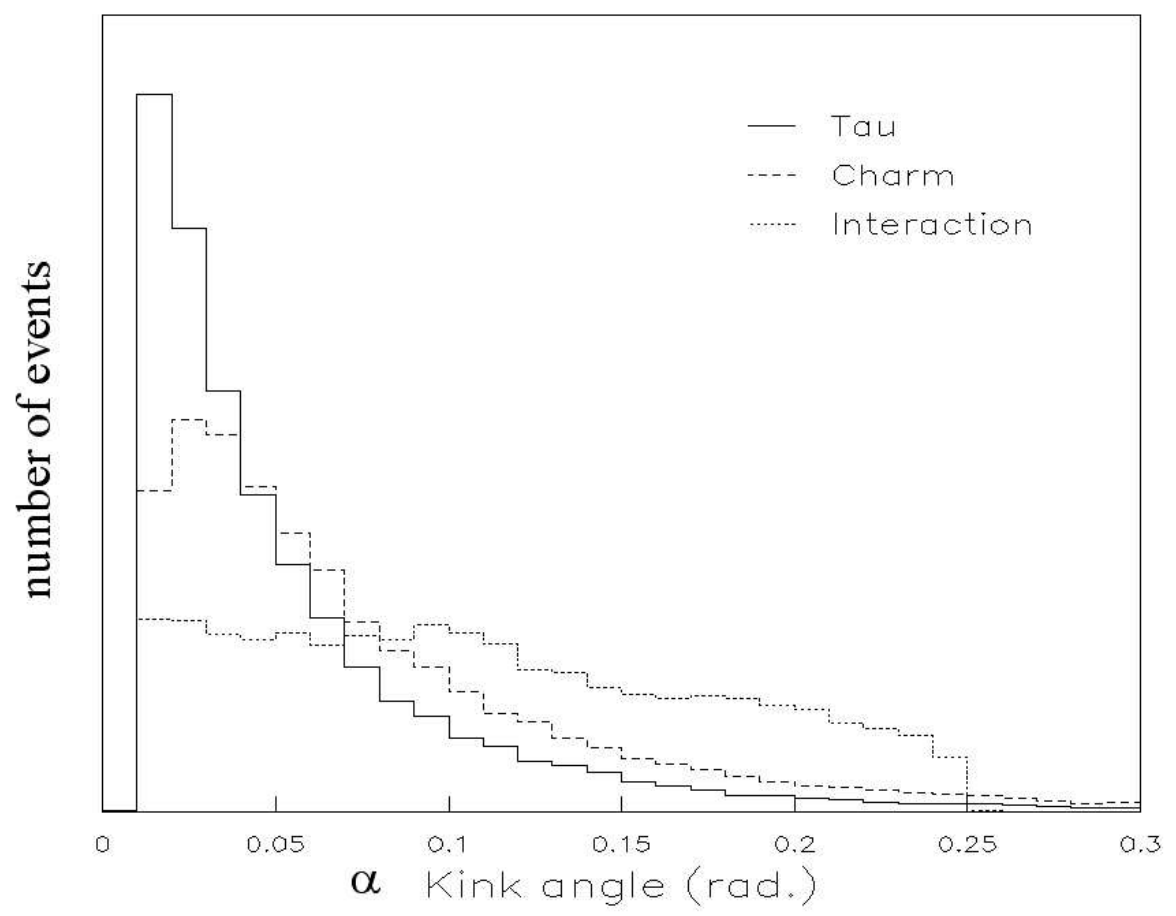

Figure 4.14: Distributions of $\alpha$ for simulated tau, charm, and interaction events. The kink angle provided significant distinction between the tau and interaction events. Large kinks were more probable for interaction events than for tau events because there were many more low momentum hadrons. 


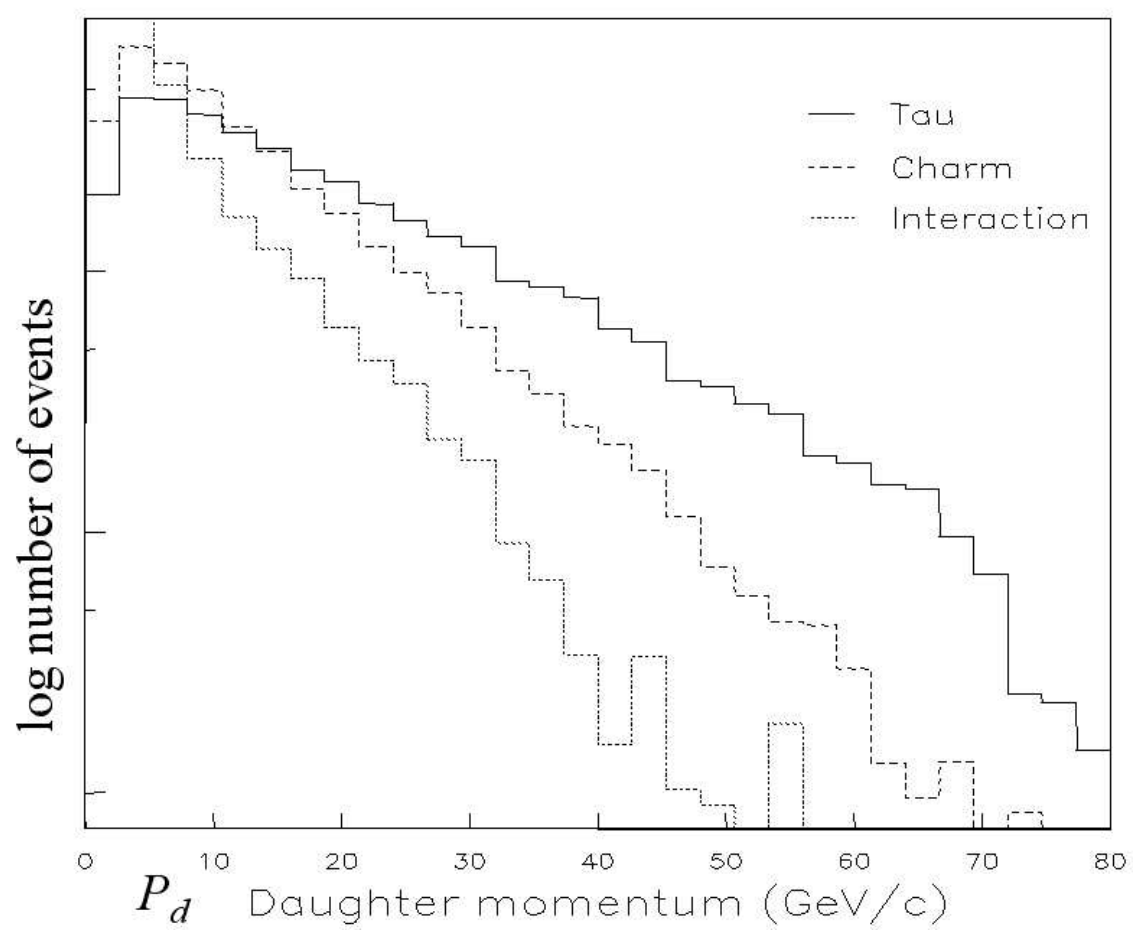

Figure 4.15: Distributions of $P_{\mathrm{d}}$ for simulated tau, charm, and interaction events. The distribution shown above does not include the three prong decays. The daughter momentum provided a distinction between the tau events and the interaction backgrounds.

momentum measurement. The daughter momentum provided a distinction between the tau events and the interaction backgrounds. The distributions of the daughter angle for simulated tau, charm, and interaction events are shown in Fig. 4.15 [29].

$\Sigma I P$ is the sum of the impact parameters of the daughter particles, which was used only for the trident events. The impact parameter was calculated using the daughter angle and the length of the tau lepton track, as shown in Fig. 4.10.

$$
I P=L \sin \alpha
$$

The sum of the impact parameters was closely related to the kink angles of the daughters and the length of the tau track. Since the tau track was generally shorter than the primary track from a hadronic interaction, the tau distribution peaked at a lower values. 


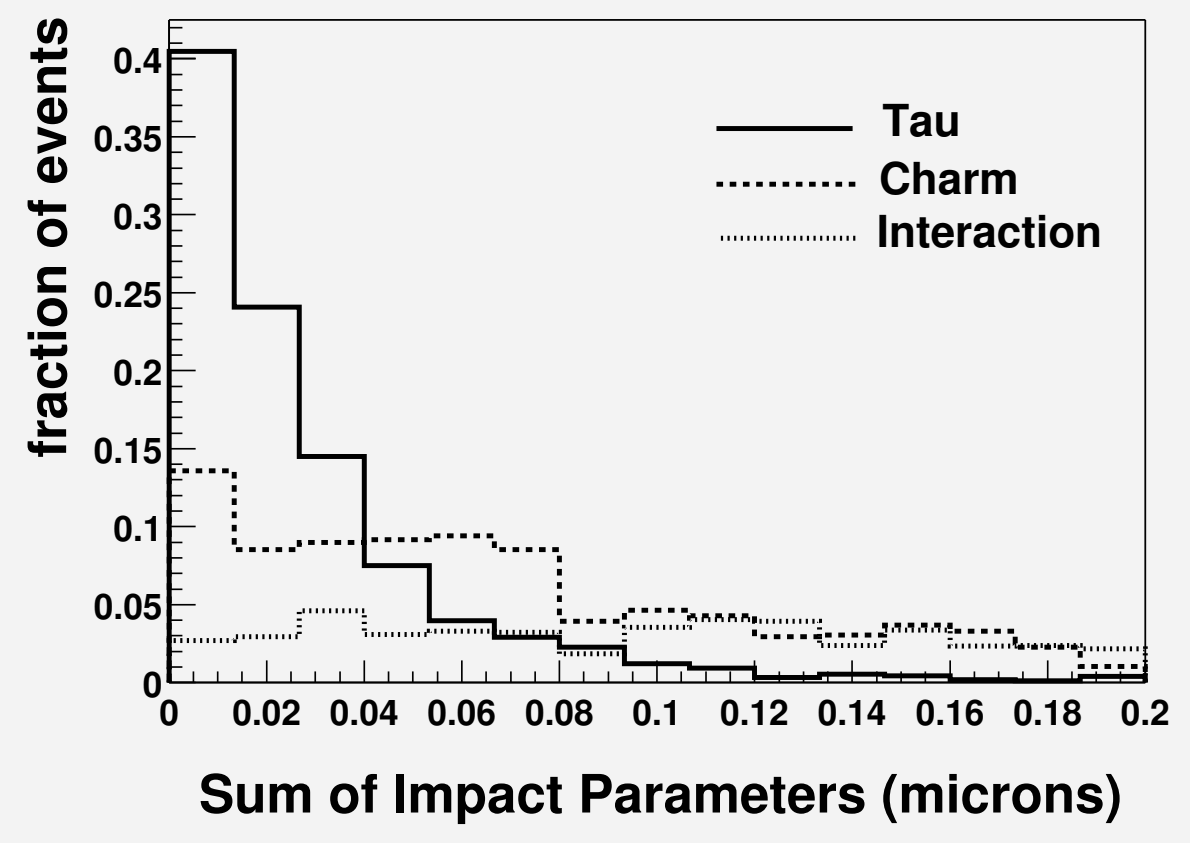

Figure 4.16: Distributions of $\Sigma I P$ for simulated tau, charm, and interaction events. The sum of the impact parameters was closely related to the kink angles of the daughters and the length of the tau track. Since the tau track was generally shorter than the primary track from a hadronic interaction, the tau distribution peaked at a lower values.

The distributions for the tau, charm, and interaction events are shown in Fig. 4.16.

\subsection{Event Classification}

Each of the 539 located neutrino interactions was classified as a charged-current or neutralcurrent interaction. Each charged-current interaction was classified by the flavor of the neutrino which interacted, identified by the charged lepton resulting from the interaction. The neutral-current interactions had a neutrino in the final state, which could not be identified; therefore, these events were not be classified by flavor. If a track was not identified as an electron or a muon, it was classified as a hadron. The requirements given below for the event type classification were based on Monte Carlo studies. 
An additional set of events were the charm events. In these events a muon or electron neutrino interacted and produced a charged lepton and a charm particle. The charged charm particle decayed to one or three charged particles. The charged charm events were potentially important backgrounds to the tau neutrino interactions. A neutral charm could also be produced in the interaction of the muon or electron neutrinos. These events had different topologies than the tau events, and, thus, they were not backgrounds.

\subsubsection{Muon Neutrino Charged-Current Interactions}

The identification of muon neutrino charged-current interactions hinged on identifying the muon produced in the interaction; the muons were identified using the muon ID at the rear of the spectrometer. Each possible muon track was projected to the proportional tubes in the muon ID wall. A muon track was required to have at least four out of six possible hits, with one hit in each of the three muon ID walls, thus, passing through at least 1.5 meters of steel. If one of the primary tracks was identified as a muon, the event was classified as a muon neutrino charged-current interaction.

\subsubsection{Electron Neutrino Charged-Current Interactions}

The electron neutrino charged-current interaction produced an electron, which was identified if an electromagnetic shower was produced in the spectrometer or radiated photons were identified through the electron-positron pairs produced in the emulsion, as described in Sec. 4.3.3. In the spectrometer, electrons were identified through their electromagnetic showers. Since hadron also shower, the electron identification must differentiate between electromagnetic and hadronic showers. The shower shape is different for these two types of showers; thus, the showers were often visually differentiated. Hadronic showers tend to be more laterally spread out than electromagnetic ones. When uncertainty existed, a neural net analysis, which used spectrometer data, was used to classify the shower as electromagnetic or hadronic [41]. The neutral net analysis used the number of continuous blocks with deposited energy in the EMCAL, the average energy deposited in these blocks, the angles of the tracks which deposited the energy, and the number of hits and pulse heights in the scintillating fibers. Fig. 4.17 illustrates a charged-current electron neutrino interaction. The first figure shows the U-view in the spectrometer, and the second figure shows the face of the EMCAL for this event. 


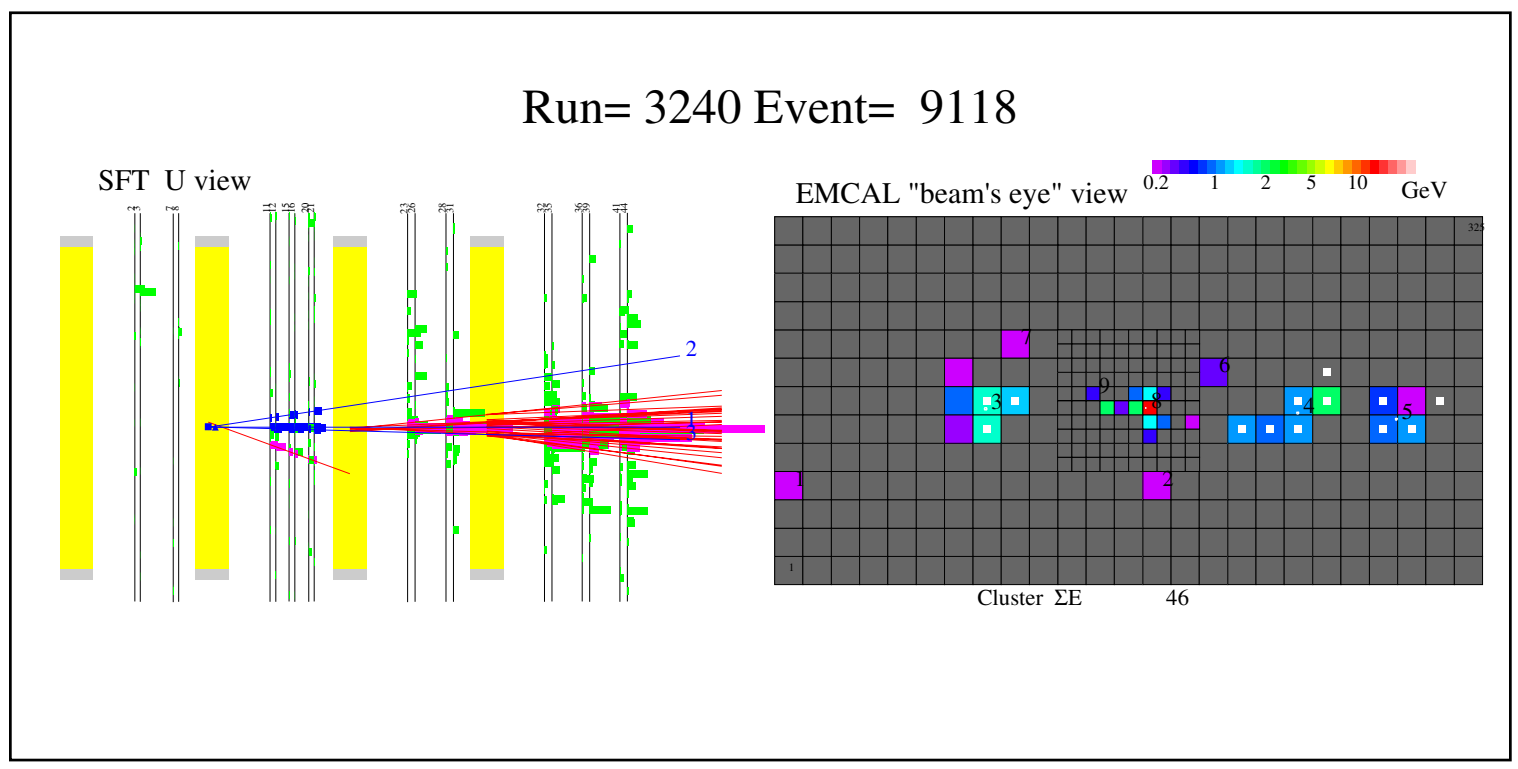

Figure 4.17: The U-view of the spectrometer data and the face of the EMCAL for a charged-current electron neutrino interaction [27]. Showering begins in the scintillating fibers before the third emulsion module. Energy deposited in the EMCAL is represented by the colored squares on the face of the EMCAL. 


\subsubsection{Tau Neutrino Charged-Current Interactions}

The tau neutrino charged-current interactions were classified by identifying the kink or trident decay of the tau lepton and were selected using emulsion data. The following requirements were necessary to identify an event as a candidate tau neutrino chargedcurrent interaction:

\section{Primary Lepton}

No muon or electron originated from the interaction vertex. If an electron (or muon) originated from the vertex, this indicated the interacting neutrino was an electron neutrino (or muon neutrino). This was applied to both the kink and trident events.

\section{Decay Length}

The parent track was less than $10 \mathrm{~mm}$ in length. The length was defined as the distance between position of the interaction vertex and the intersection of the parent and daughter

track. This requirement was restricted only by the data analysis and was designed to include all possible tau decays.

\section{Daughter Kink Angle}

The angle of the daughter track with respect to the parent track was greater than 10 mrad. This angle was calculated using a straight line fit of the last three segments of the parent track and the first three segments of the daughter track. This cut was only applied to the kink events.

\section{Daughter Impact Parameter}

The impact parameter of the daughter track to the neutrino interaction vertex was less than $500 \mu \mathrm{m}$. The impact parameter was calculated by projecting the daughter track back to the neutrino interaction vertex and calculating the shortest distance between the tracks. This cut was only applied to the kink events.

\section{Daughter Momentum}

The daughter track momentum, $P_{\mathrm{d}}$, was greater than $1 \mathrm{GeV} / \mathrm{c}$ for single prong decays. The momentum of the daughter was measured either by the deflection of the track by the analysis magnet or by multiple scattering in successive emulsion plates within a module. 
If a kink or trident vertex satisfies these requirements, the event was classified as a tau neutrino charged-current interaction candidate, and a statistical analysis was performed on the event.

\subsubsection{Charm Particles}

Charged charm particles, produced in either an electron neutrino or muon neutrino chargedcurrent interaction, decayed to one or three charged particles. If the primary lepton was not identified, the event was a potential background to the tau events. The statistical analysis, described in the next chapter, studied these backgrounds. If a muon or electron originating from the primary was identified with a decay, the event was rejected as a tau neutrino candidate and classified as a charged charm candidate. The charged charm candidates are described in more detail in Sec. 5.3; in this section, the expected number of charm candidates and the probability that a charm decay could pass the tau decay criteria are discussed.

Neutral charm particles were also produced in electron neutrino and muon neutrino charged-current interactions. The neutral charm particles usually decayed to two charged particle; this type of decay was referred to as a "vee decay". The neutral charm particles may have decayed to zero, four, or more charged particles. Neutral charm particles were identified by these decays. Since the tau lepton decays to one or three charged particles, the neutral decays were not backgrounds for the tau, nor were any tau lepton decays misidentified as neutral decays.

\subsubsection{Neutral-Current Interaction}

The neutral-current interactions were not classified by flavor, as the final state lepton was a neutrino, which was undetectable. The neutral current events had no distinct event topologies which allowed their definitive identification. If a neutrino interaction had no identified primary lepton, it was classified as a neutral-current event.

\subsection{Number of Events}

After applying the sets of criteria explained above to all located neutrino interactions, an initial hypothesis of the composition of the data set was determined. The resulting composition is summarized in Table 4.3. The data set, used to measure the charged-current cross section of the tau neutrino in the deep inelastic scattering region, only included deep 


\section{Composition of data Set}

\begin{tabular}{|c|c|c|c|}
\hline \multicolumn{3}{|c|}{ Neutral Current } & 163 \\
\hline \multicolumn{3}{|c|}{ Charged Current } & 376 \\
\hline \multicolumn{2}{|l|}{$\nu_{\mu}$} & 214 & \\
\hline \multicolumn{2}{|l|}{$\nu_{e}$} & 153 & \\
\hline \multicolumn{2}{|l|}{$\nu_{\tau}$} & 9 & \\
\hline$\nu_{\tau}$ long & 7 & & \\
\hline$\nu_{\tau}$ short & 2 & & \\
\hline \multicolumn{2}{|l|}{ Charm } & 10 & \\
\hline Charged & 4 & & \\
\hline Neutral & 6 & & \\
\hline \multicolumn{3}{|c|}{$\frac{1}{\text { Total }}$} & 539 \\
\hline
\end{tabular}

Table 4.3: Composition of the data set separated by neutral-current or charged-current interactions. The charged-current interactions were further separated by flavor. The tau candidates were classified as a long or a short decay. Note the charm events are a subset of the muon neutrino and electron neutrino charged-current events.

inelastic scattering interactions. Some charged-current electron neutrino interactions were in the quasi-elastic scattering region; hence these events were not used. The quasi-elastic events were distinctive because they generally had only one primary track, the electron, although the possibility of a recoil proton existed. Events with this topology were not used in the measurement.

A total of 539 events were located. Of those, 163 were classified as neutral-current interactions, and 376 were classified as charged-current interactions. Of the chargedcurrent interactions, 214 were identified as muon-neutrino interactions; 153 were identified as electron neutrino interactions; 9 were tau neutrino interaction candidates. Of the nine tau neutrino interaction candidates, seven were long decays ${ }^{1}$ and two were short decays. Charm candidates occurred in 10 of the muon and electron neutrino interactions. Of these 10 events, 4 included charged charm particles, and 6 included neutral charm.

\footnotetext{
${ }^{1}$ Long decays occurred when the tau lepton traversed at least one emulsion layers. Short decays occurred when the tau lepton decayed before it reached the emulsion.
} 


\section{Chapter 5}

\section{Tau and Charged Charm Candidates}

Seven of the 539 neutrino events fulfilled the requirements for a tau neutrino chargedcurrent interaction described in Sec. 4.6.3. Four of the events fulfilled the charged charm requirements described in Sec. 4.6.4. Each tau and charged charm candidate is explored in more detail in this chapter. Figures depicting each of the events's spectrometer data and emulsion data are included in Sec. 5.1 and Sec. 5.2. The emulsion figures show the track segments in the emulsion; tracks are formed using these segments. These tracks are then projected into the spectrometer. The spectrometer pictures include the $\mathrm{X}$-view, $\mathrm{U}$-view, and $\mathrm{V}$-view. The $\mathrm{X}$-view shows all components of the spectrometer. The Uview and $\mathrm{V}$-view show only the scintillating fibers and the emulsion modules. The dark spots in the U-view and V-view are scintillating fiber hits fitted to the emulsion track, which indicate the track is high momentum and the angle did not change significantly between the emulsion modules and the scintillating fibers. The dashed lines in the spectrometer pictures are short tracks, defined as tracks with three or fewer track segments in the emulsion. Since these tracks did not leave the emulsion, there was no activity in the spectrometer along these tracks. The available multiple scattering and spectrometer momentum measurements are given for each of the tracks. 
Parameters of Tau Candidate 3024_30175

\begin{tabular}{|c|c|c|c|c|}
\hline$\theta(\mathrm{rad})$ & $\Delta \phi(\mathrm{rad})$ & $L(\mathrm{~mm})$ & $\alpha(\mathrm{rad})$ & Decay Material \\
\hline 0.028 & 1.09 & 4.59 & 0.093 & emulsion \\
\hline
\end{tabular}

Table 5.1: Tau kink candidate 3024_30175.

Track Information for Tau Candidate 3024_30175

\begin{tabular}{|c|c|c|c|c|}
\hline & \multicolumn{2}{|c|}{ Momentum $(\mathrm{GeV})$} & \multicolumn{2}{|c|}{ Identification } \\
\hline Track & MS & Spec & Particle & \\
\hline 1 & $0.42_{-0.1}^{+0.2}$ & & hadron & \\
2 & $2.9_{-0.7}^{+1.5}$ & & electron & daughter \\
3 & $4.0_{-1.6}^{+6.5}$ & & hadron & \\
4 & & & tau & parent \\
\hline
\end{tabular}

Table 5.2: Tau candidate 3024_30175 has three primary tracks. Track 4, the tau candidate, decays to track 2 , an electron identified in the emulsion. None of the tracks are unambiguously fit to hits in the drift chambers or EMCAL; therefore, there are no spectrometer momentum measurements for these tracks.

\subsection{Tau Candidates}

\section{Tau Candidate 3024_30175}

In tau candidate 3024_30175, a neutrino interacted in the first emulsion module and produced three charged primary tracks. One of these tracks, track 4, is a candidate tau track which decayed to an electron, track 2 . The electron was identified in the emulsion. The other primary tracks, tracks 1 and 3 , are hadrons. The parameters of this event are summarized in Table 5.1. Only multiple scattering momentum measurements were available and are listed in Table 5.2. Fig. 5.1, 5.2, and 5.3 show the $\mathrm{X}$-view, U-view, and V-view of the spectrometer. As best seen in the $\mathrm{V}$-view, tracks 1 and 2 match with hits in the spectrometer. Track 2 begins to shower after passing through the second emulsion module. Tracks 2 and 4 overlap in the U-view, but not in the X- or V-view. Fig. 5.4 shows the emulsion data for this event. 


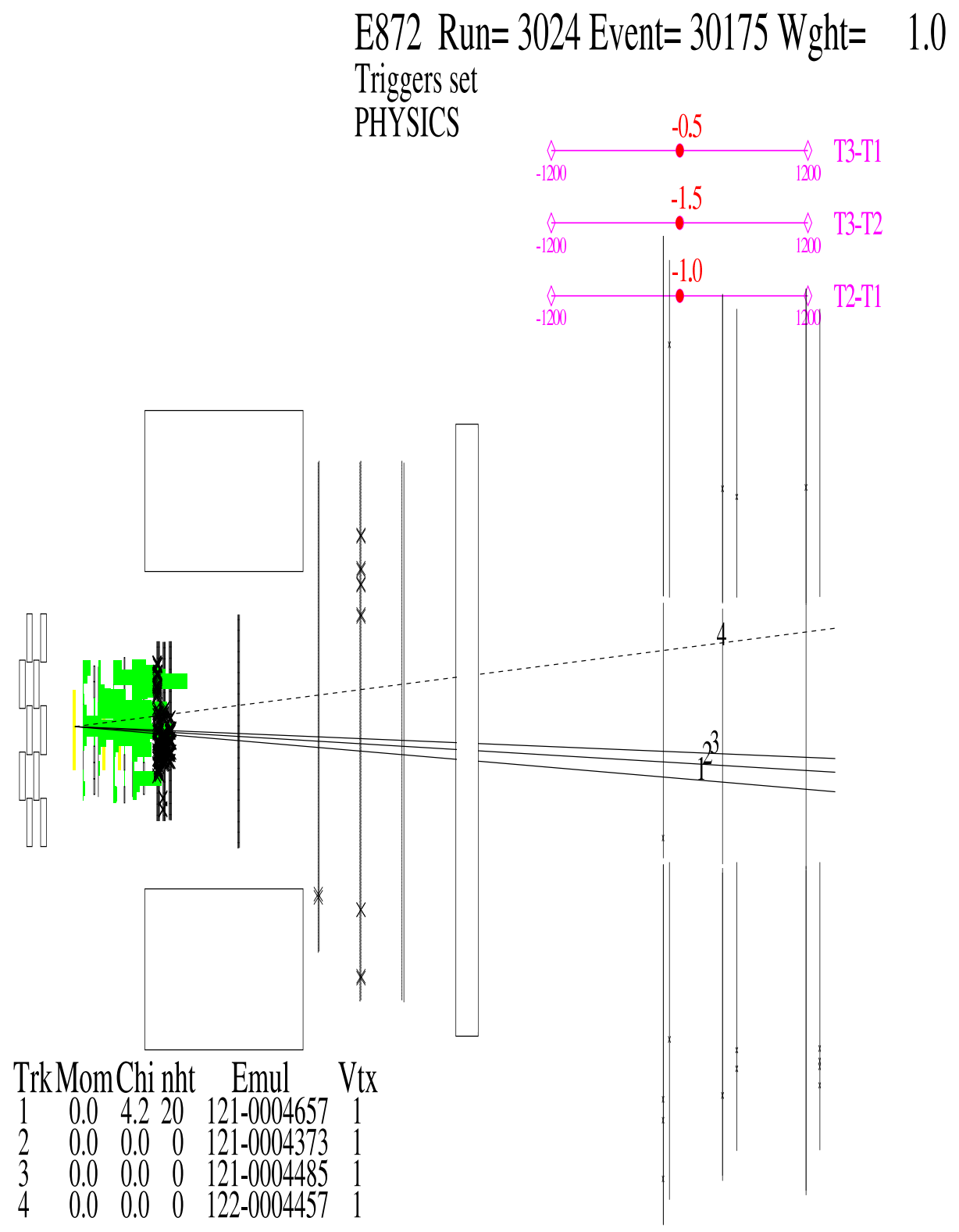

Figure 5.1: The X-view in the spectrometer for tau candidate 3024_30175. The tracks shown are the projections of the emulsion tracks onto the spectrometer. Tracks 1 and 3 are charged primary hadrons. Track 4 is the tau track candidate, and track 2 is the daughter electron, identified in the emulsion. 


\section{E872 Run= 3024 Event= 30175 Wght= 1.0 U View}

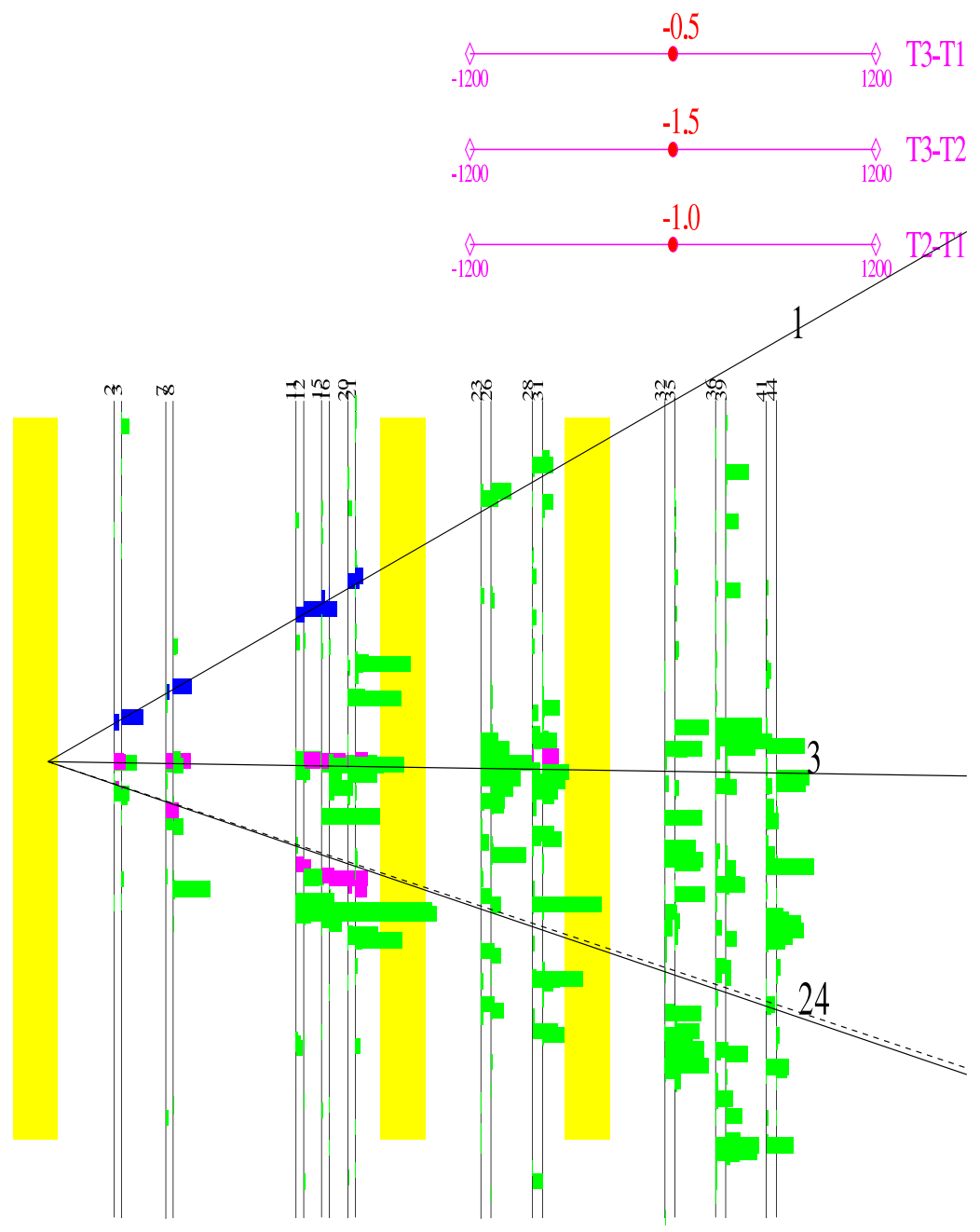

Figure 5.2: The U-view in the spectrometer for tau candidate 3024_30175. The darker hits are scintillating fiber hits matched to the emulsion tracks. Track 4 is the tau candidate, and track 2 is the daughter electron. These two tracks overlap in this view, but not in the $\mathrm{X}$ - or $\mathrm{V}$-views. The other two tracks are hadrons. 


\section{E872 Run= 3024 Event $=30175$ Wght= 1.0 V View}

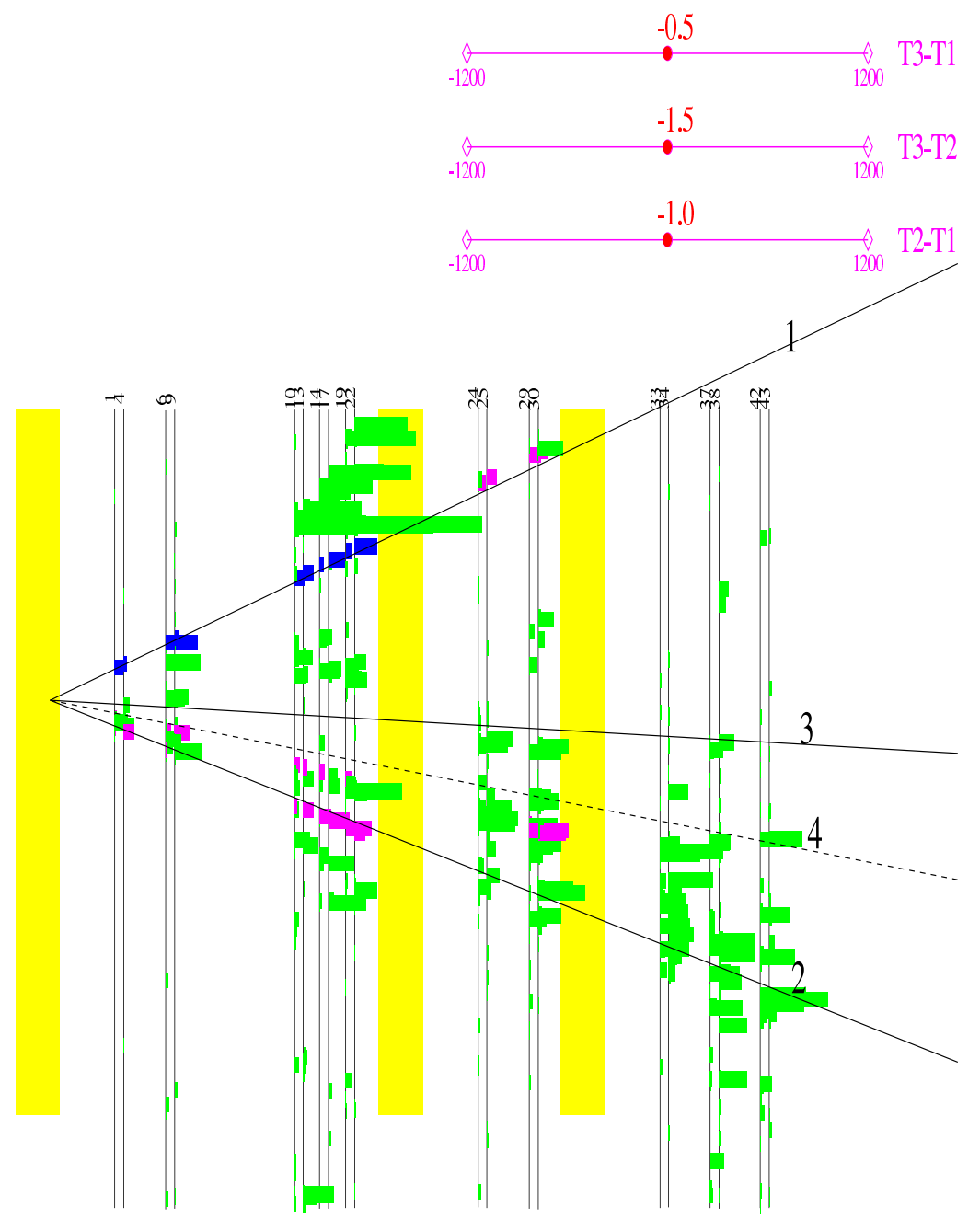

Figure 5.3: The V-view in the spectrometer for tau candidate 3024_30175. 


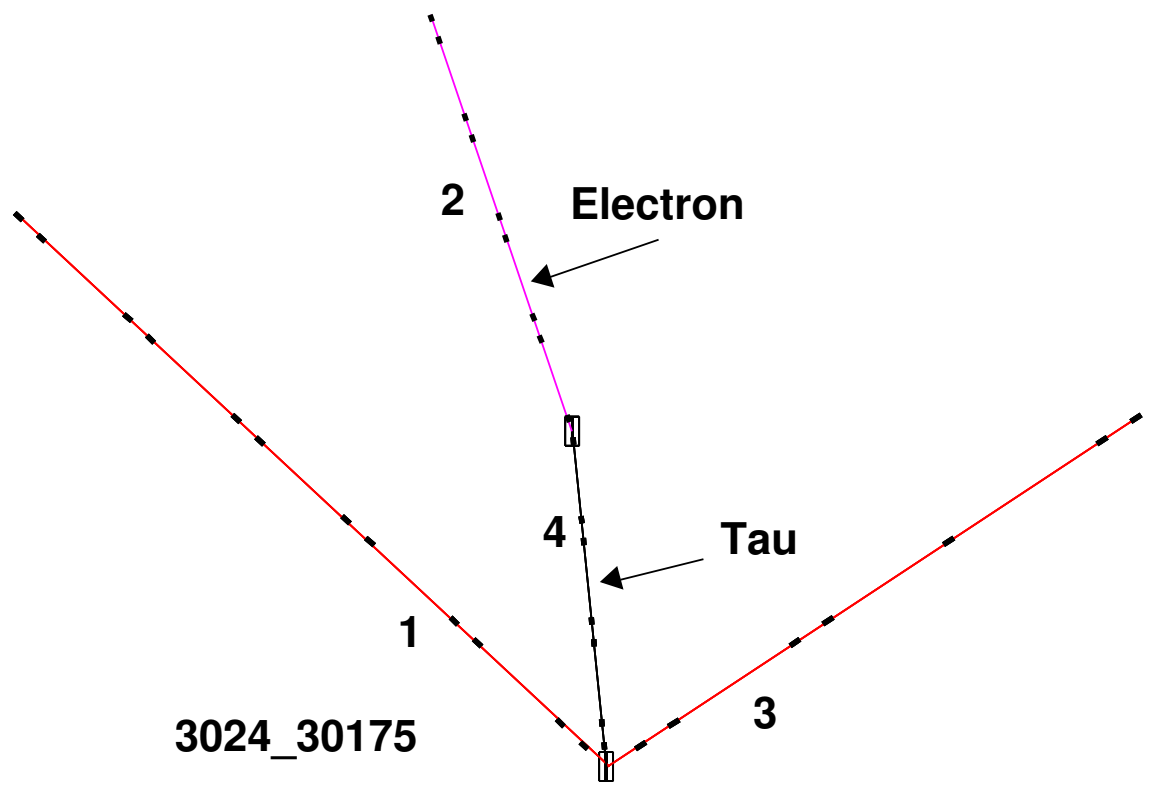

Figure 5.4: The neutrino interaction produced three primary charged tracks. Tracks 1 and 3 are hadrons. Track 4 is the tau candidate, and track 2 is the electron daughter. The parameters and momentum measurements for this event are listed in Table 5.1 and 5.2. This neutrino interaction occurred in ECC200 type emulsion, and the decay occurred in the emulsion. 
Parameters of Tau Candidate 3030_01910

\begin{tabular}{|c|c|c|c|c|}
\hline$\theta(\mathrm{rad})$ & $\Delta \phi(\mathrm{rad})$ & $L(\mathrm{~mm})$ & $\alpha(\mathrm{rad})$ & Decay Material \\
\hline 0.067 & 2.71 & 0.29 & 0.090 & emulsion \\
\hline
\end{tabular}

Table 5.3: Tau kink candidate 3039_01910.

Track Information for Tau Candidate 3039_01910

\begin{tabular}{|c|c|c|c|c|}
\hline 3039_01910 & \multicolumn{2}{|c|}{ Momentum $(\mathrm{GeV})$} & \multicolumn{2}{|c|}{ Identification } \\
\hline Track & MS & Spec & Particle & \\
\hline 1 & $4.6_{-0.4}^{+1.4}$ & & hadron & daughter \\
2 & & $19 \pm 4$ & hadron & \\
3 & $19.5_{-}^{+}$ & & hadron & \\
4 & $7.9_{-1.9}^{+3.3}$ & & hadron & \\
5 & $1.56_{-0.5}^{+1.3}$ & & hadron & \\
6 & & & & tau \\
\hline
\end{tabular}

Table 5.4: Tau candidate 3039_01910 has five primary tracks. The tau candidate track, track 6 , decayed or interacted to produce a hadron, track 1. Track 2 traversed the EMCAL which provided a spectrometer momentum measurement.

\section{Tau Candidate 3039_01910}

In tau candidate 3039_01910, a neutrino interacted in the first emulsion module and produced five charged primary tracks. The tau candidate track, track 6 , decayed or interacted to produce a hadron, track 1 . The other primary tracks are hadrons. The parameters of this event are summarized in Table 5.3. Multiple scattering momentum measurements were available for all tracks but one, which had a spectrometer momentum measurement; all measurements are listed in 5.4. Fig. 5.5, 5.6, and 5.7 show the X-view, U-view, and $\mathrm{V}$-view of the spectrometer. There is activity in the scintillating fibers, the drift chambers, and the EMCAL, as shown in the X-view. The total hadronic energy in this event is $62.3 \pm 7 \mathrm{GeV}$, as measured in the EMCAL. Fig. 5.6 and 5.7 show hadronic showering which occurs in the scintillating fibers. Fig. 5.8 shows the emulsion data for this event, and Fig. 5.9 shows a close up of the vertex in the emulsion. 


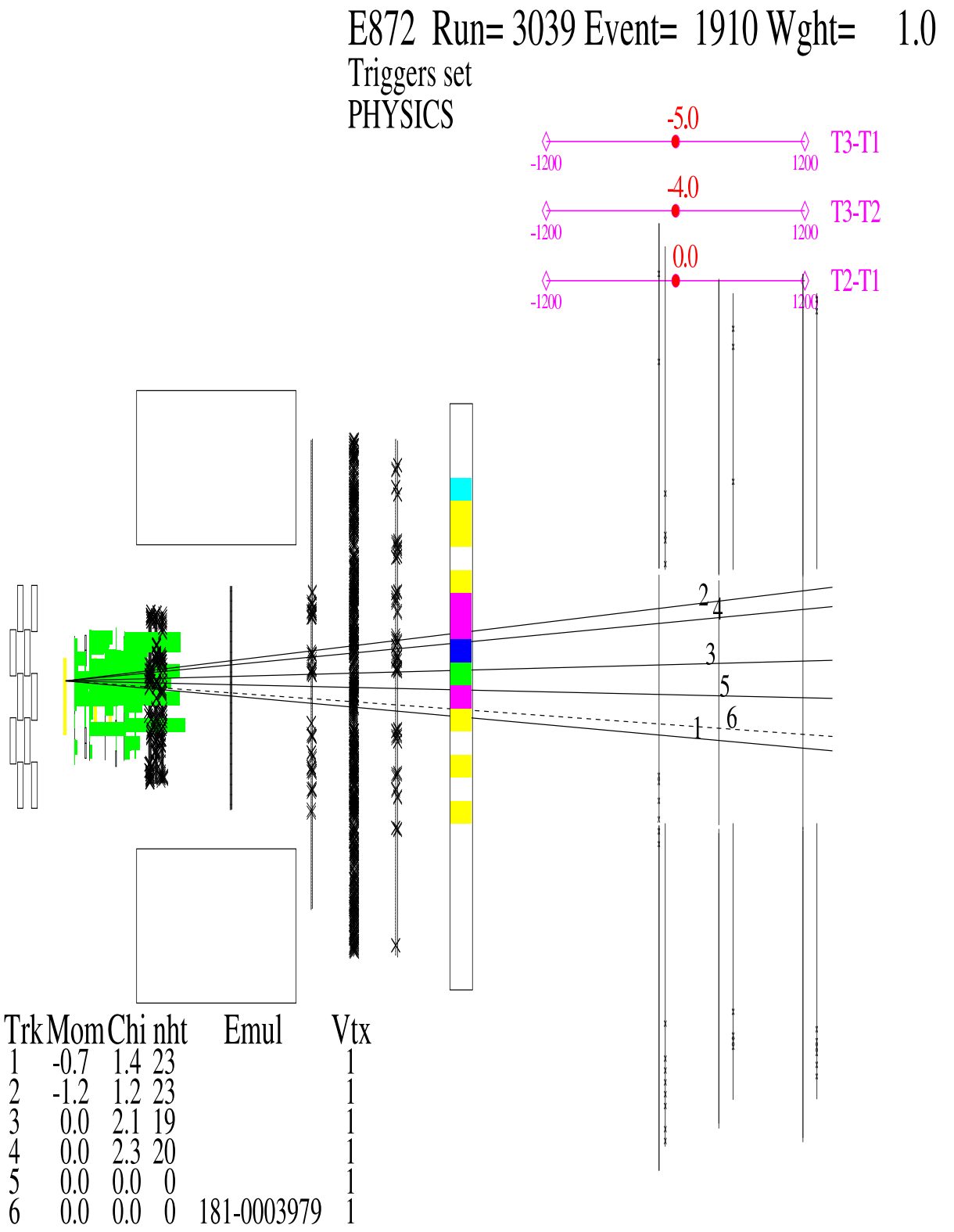

Figure 5.5: The X-view in the spectrometer for tau candidate 3039_01910. The tracks shown are the projections of the emulsion tracks onto the spectrometer. Tracks 2-5 are primary hadrons. Track 6 is the candidate tau track, and track 1 is the daughter hadron. These tracks produced activity in the scintillating fibers, drift chambers, and EMCAL. The total hadronic energy in this event is $62.3 \pm 7 \mathrm{GeV}$, as measured in the EMCAL. 


\section{E872 Run= 3039 Event= 1910 Wght= 1.0 U View}
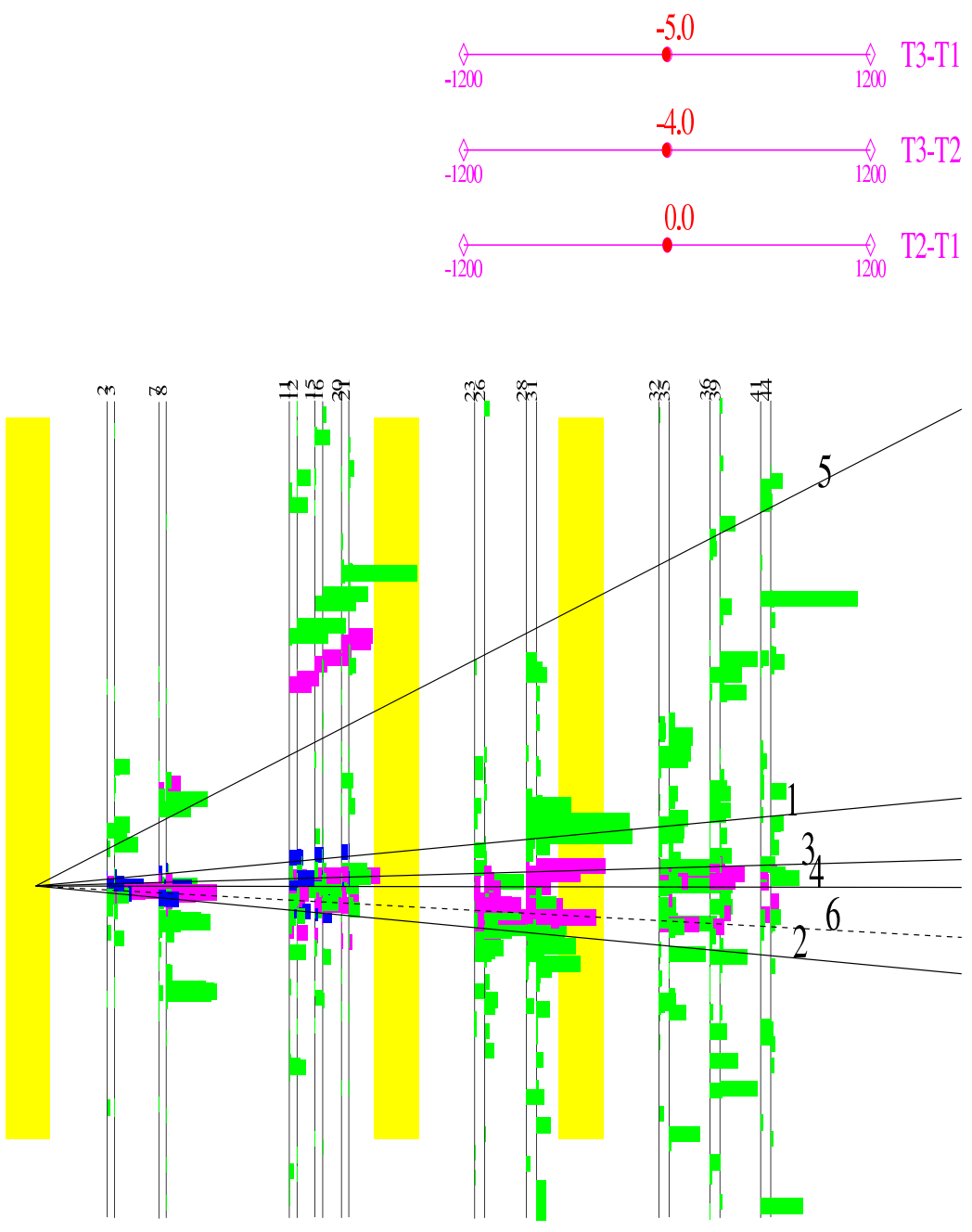

Figure 5.6: The U-view in the spectrometer for tau candidate 3039_01910. Tracks 2-5 are primary hadrons. Track 6 is the candidate tau track, and track 1 is the daughter hadron. In this interaction, a hadronic shower developed in the scintillating fibers. 


\section{E872 Run=3039 Event= 1910 Wght= 1.0 V View}

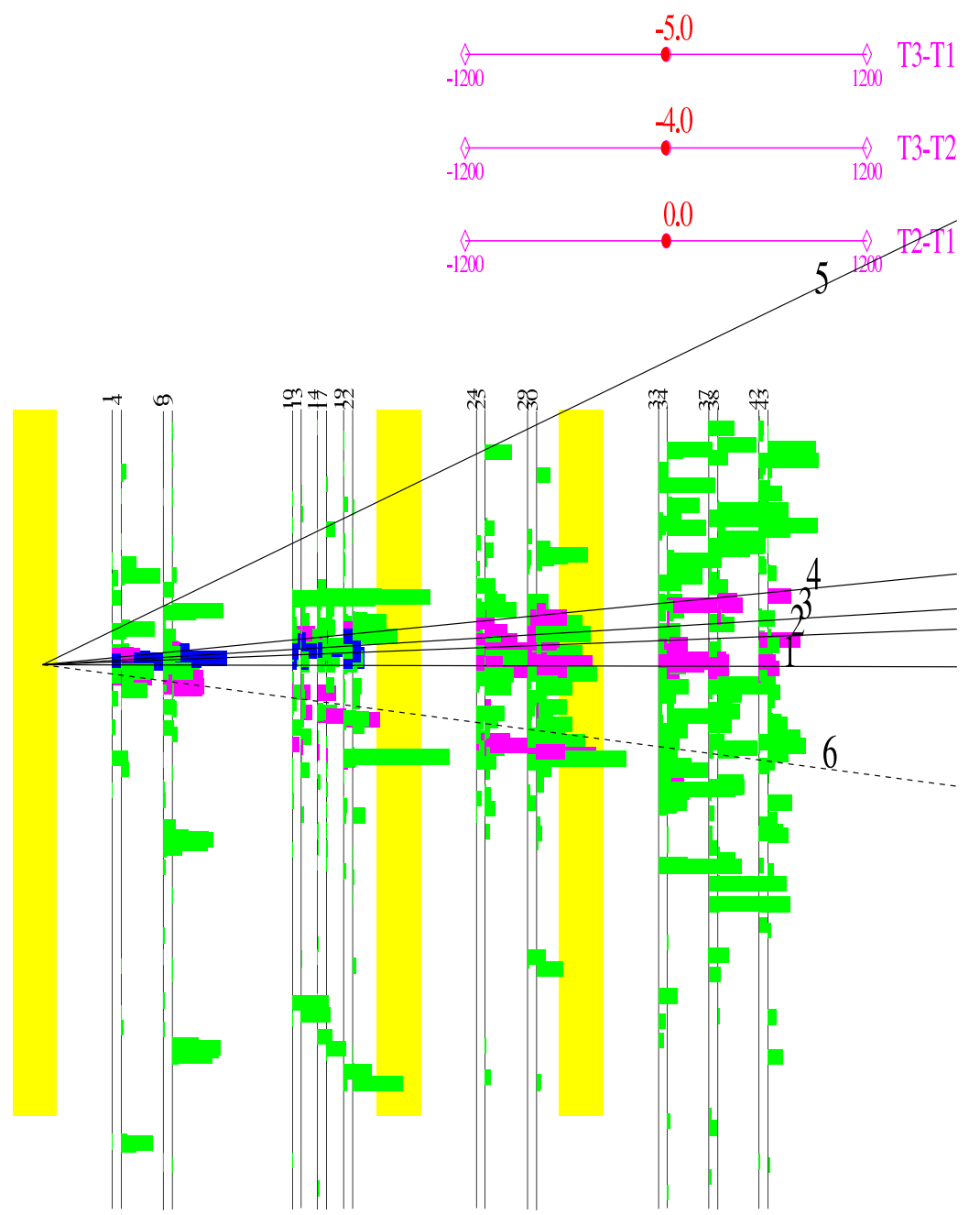

Figure 5.7: The V-view in the spectrometer for tau candidate 3039_01910. 


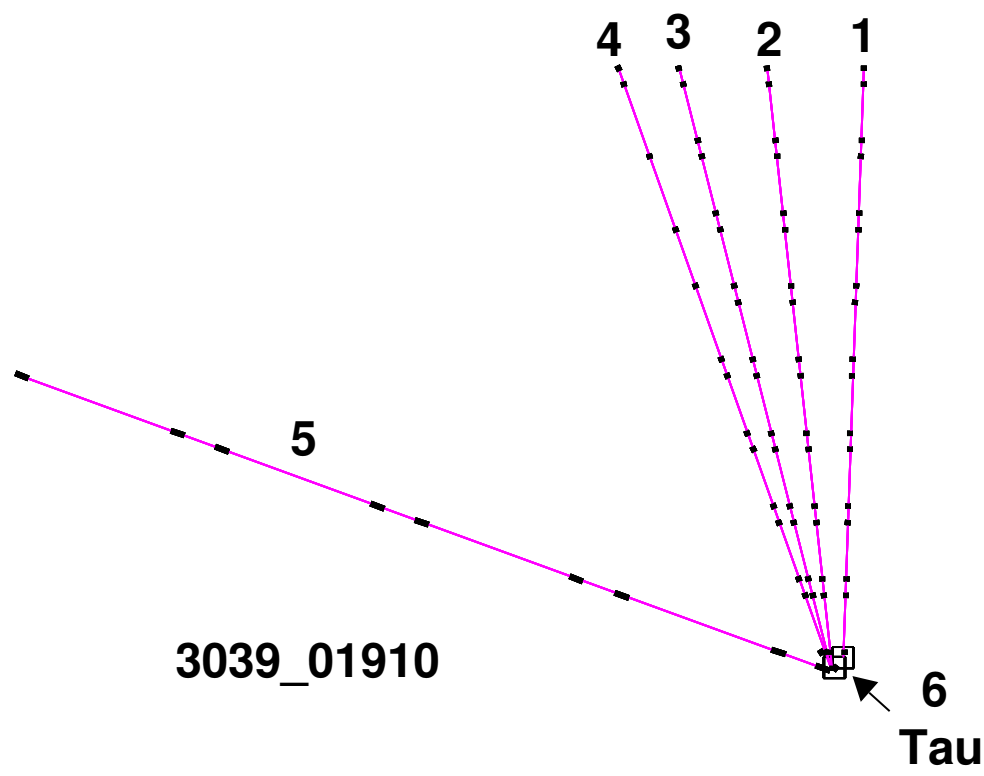

Figure 5.8: The emulsion data for tau candidate 3039_01910. All tracks are hadrons except for the track 6 , the candidate tau track. The parameters and momentum measurements for this event are listed in Table 5.3 and 5.4. The neutrino interaction occurred in ECC200 type emulsion, and the decay occurred in emulsion. 


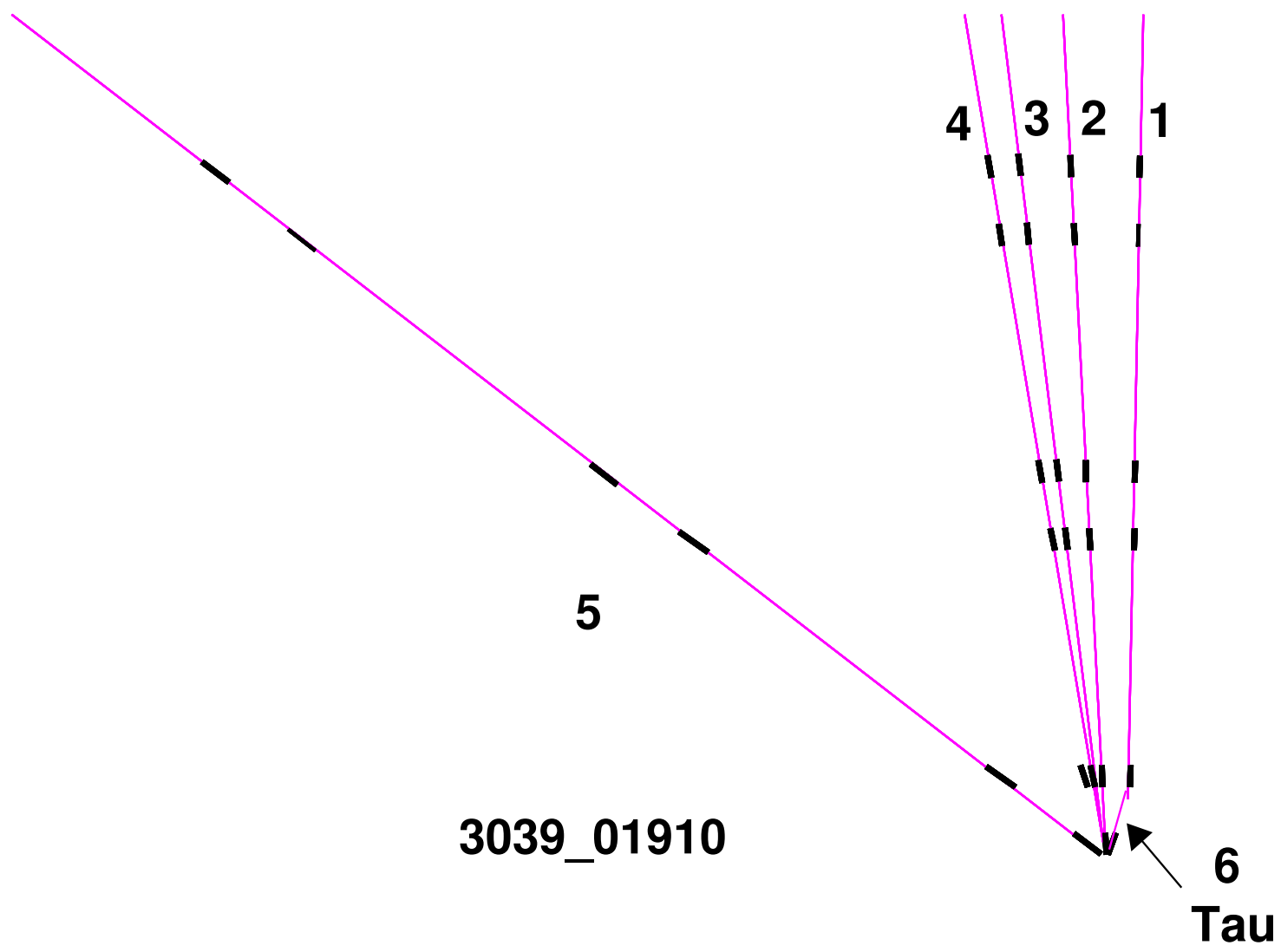

Figure 5.9: A close-up of the vertex in the emulsion data for tau candidate 3039_01910. 
Parameters of Tau Candidate 3263_25102

\begin{tabular}{|c|c|c|c|c|}
\hline$\theta(\mathrm{rad})$ & $\Delta \phi(\mathrm{rad})$ & $L(\mathrm{~mm})$ & $\alpha(\mathrm{rad})$ & Decay Material \\
\hline 0.169 & 0.17 & 1.97 & 0.130 & steel \\
\hline
\end{tabular}

Table 5.5: Tau kink candidate 3263_25102.

Track Information for Tau Candidate 3263_25102

\begin{tabular}{|c|c|c|c|c|}
\hline 3263_25102 & \multicolumn{2}{|c|}{ Momentum $(\mathrm{GeV})$} & \multicolumn{2}{|c|}{ Identification } \\
\hline Track & MS & Spec & Particle & \\
\hline 2 & $4.0_{-0.9}^{+1.6}$ & & hadron & \\
3 & $1.0_{-0.2}^{+0.3}$ & & hadron & \\
4 & $1.9_{-0.7}^{+2.2}$ & & hadron & daughter \\
5 & & & tau & parent \\
\hline
\end{tabular}

Table 5.6: Tau candidate 3263_25102 has three charged primary tracks. The tau candidate, track 5, decayed or interacted to produce a single charged hadron, track 4 . The other two primary tracks are hadrons. None of the tracks are unambiguously fit to hits in the drift chambers or EMCAL; therefore, there are no spectrometer momentum measurements for these tracks.

\section{Tau Candidate 3263_25102}

In tau candidate 3263_25102, a neutrino interacted in the first emulsion module and produced three charged primary tracks. The tau candidate, track 5 , decayed or interacted to produce a single charged hadron, track 4 . The other two primary tracks are hadrons. The parameters of this event are summarized in Table 5.5. Only multiple scattering momentum measurements were available and are listed in Table 5.6. Fig. 5.10, 5.11, and 5.12 show the $\mathrm{X}$-view, U-view, and $\mathrm{V}$-view of the spectrometer. There is activity in the scintillating fibers, the drift chambers, and the EMCAL. The hadronic energy of this event is $5.5 \pm 0.8$ $\mathrm{GeV}$, as measured in the EMCAL. There is some hadronic showering along track 3 and track 4 in the scintillating fibers. Fig. 5.13 shows the emulsion data for this event. 


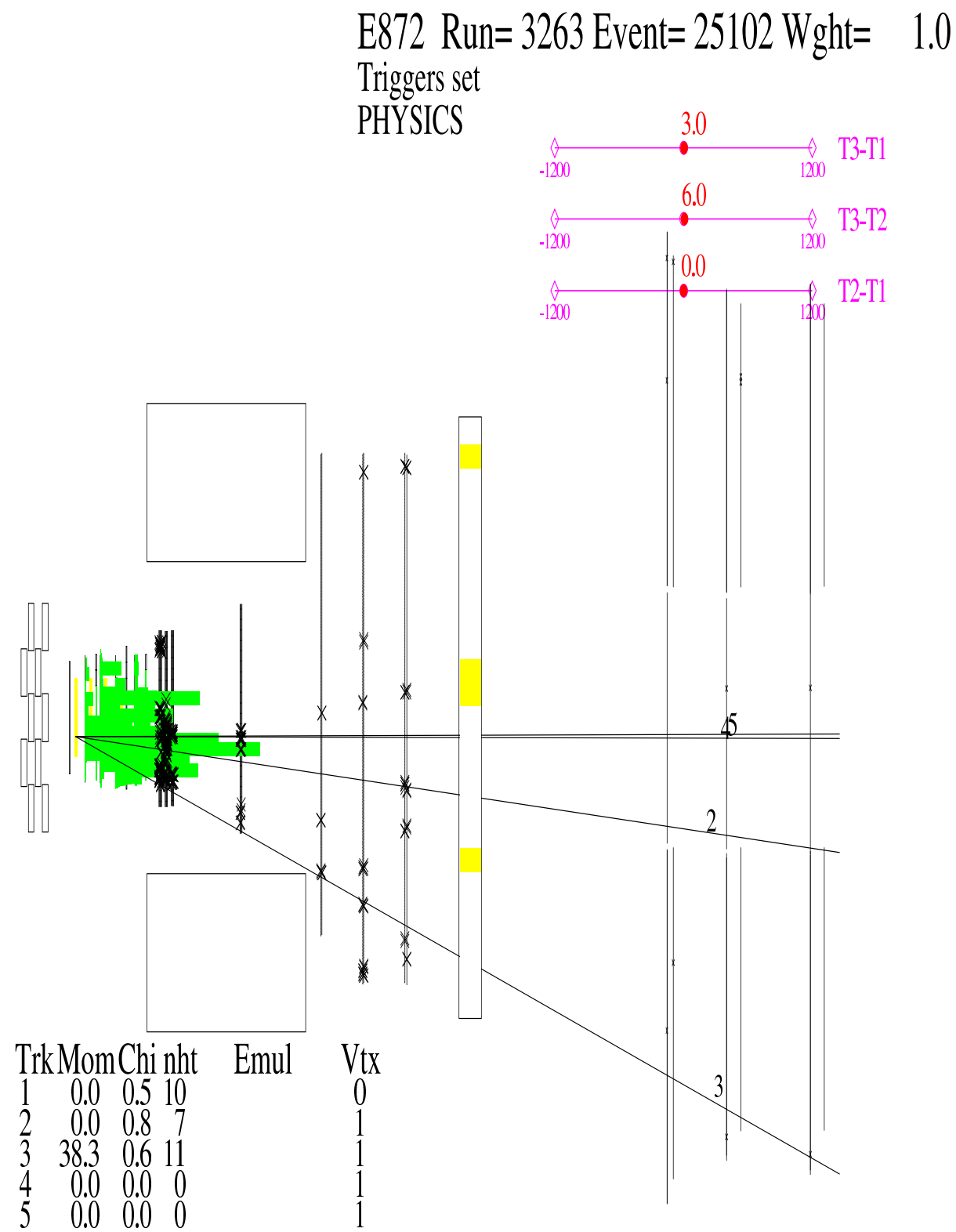

Figure 5.10: The X-view in the spectrometer for tau candidate 3263_25102. The tracks shown are the projections of the emulsion tracks onto the spectrometer. Track 2 and track 3 are primary hadrons. Track 5 is the candidate tau track which decayed to or interacted to produce a charged hadron, track 4 . The tracks produced activity in the scintillating fibers, the drift chambers, and the EMCAL. The hadronic energy of this event is $5.5 \pm 0.8$ $\mathrm{GeV}$, as measured in the EMCAL. Tracks 4 and 5 overlap in this view, but not in the Uor $\mathrm{V}$-view. 


\section{E872 Run= 3263 Event= 25102 Wght= 1.0 U View}
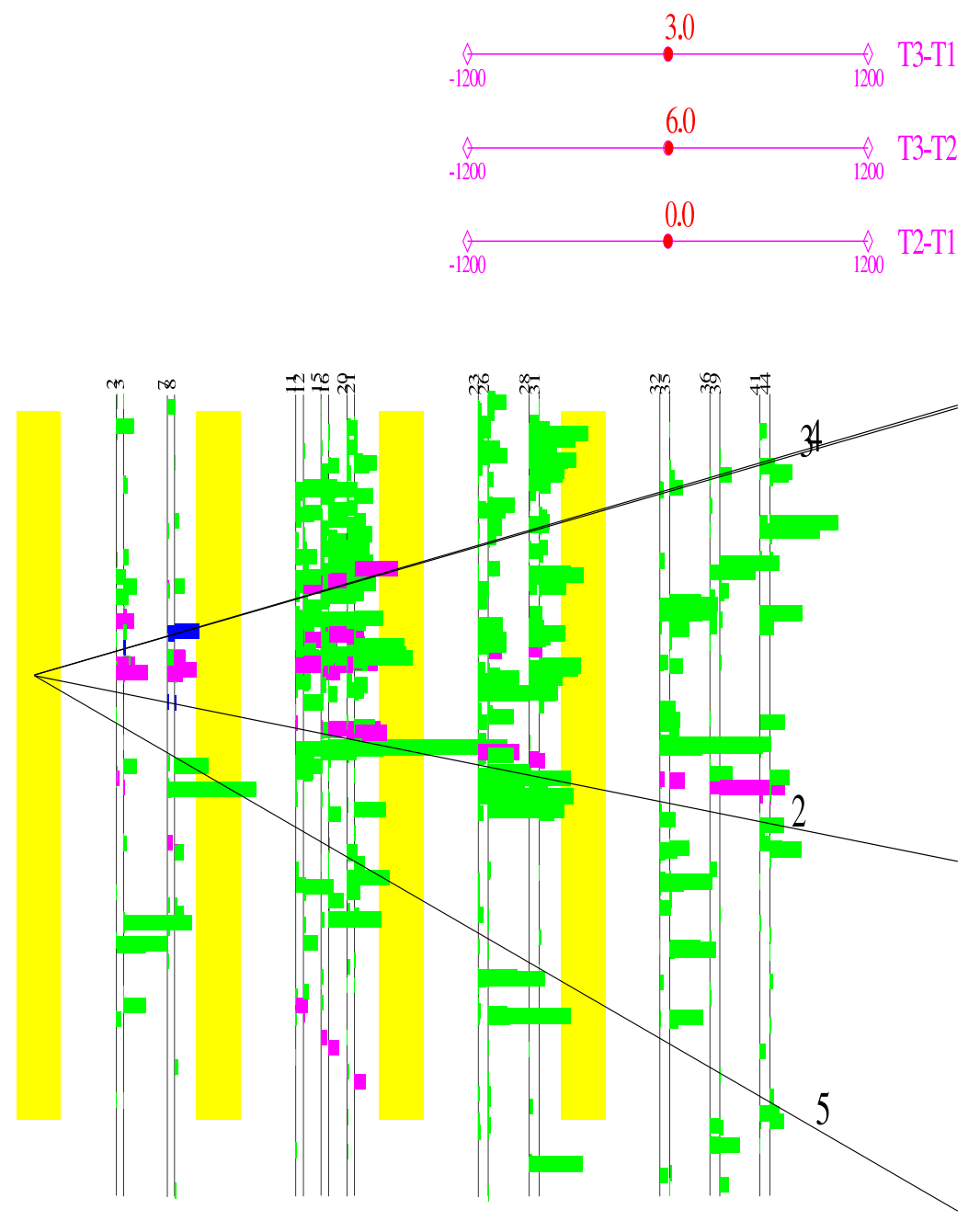

Figure 5.11: The U-view in the spectrometer for tau candidate 3263_25102. Track 2 and 3 are primary hadrons. Track 5 is the candidate tau track which decayed to or interacted to produce a charged hadron, track 4 . There is some hadronic showering along track 3 and track 4 . Tracks 3 and 4 overlap in this view, but not in the others. 


\section{E872 Run= 3263 Event= 25102 Wght= 1.0 V View}
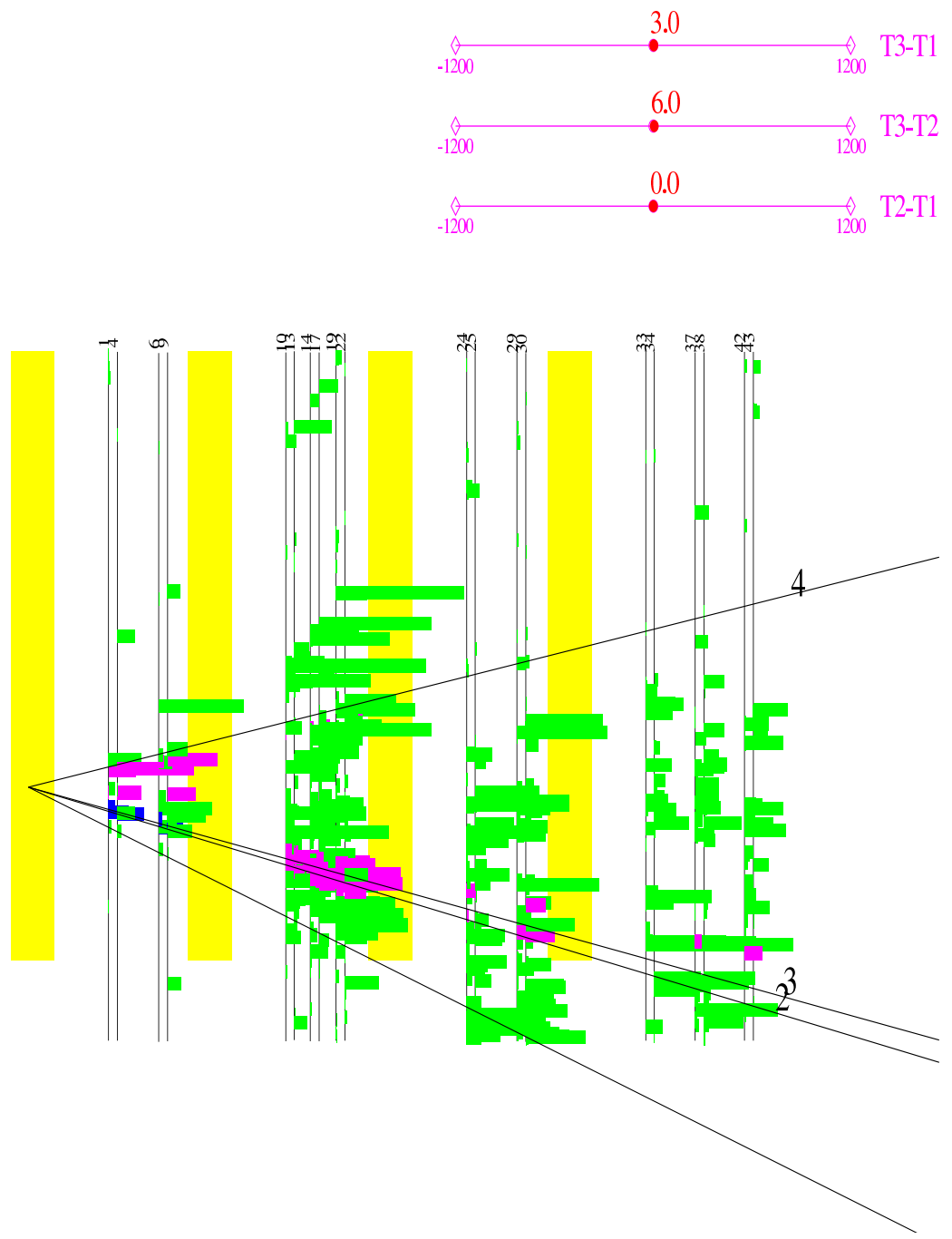

Figure 5.12: The V-view in the spectrometer for tau candidate 3263_25102. The label for track 5 , the bottom track, is to the left of the track in this view. 


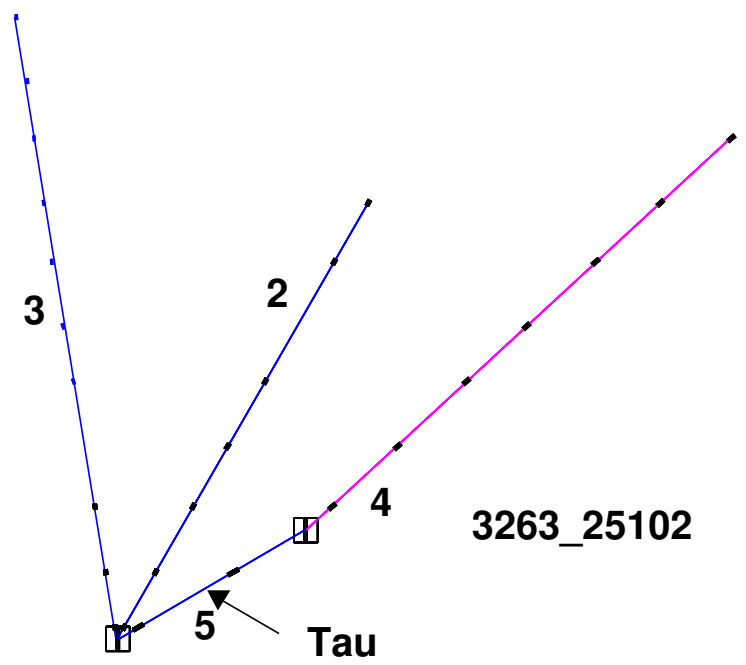

Figure 5.13: The emulsion data for tau candidate 3263_25102. Tracks 2 and 3 are charged hadrons. Track 5 is the candidate tau track which decayed to or interacted to produce track 4, a hadron. The parameters and momentum measurements for this event are listed in Table 5.5 and 5.6. The neutrino interaction occurred in ECC800, and the decay occurred in steel. 
Parameters of Tau Candidate 3333_17665

\begin{tabular}{|c|c|c|c|c|}
\hline$\theta(\mathrm{rad})$ & $\Delta \phi(\mathrm{rad})$ & $L(\mathrm{~mm})$ & $\alpha(\mathrm{rad})$ & Decay Material \\
\hline 0.016 & 2.84 & 0.55 & 0.013 & emulsion \\
\hline
\end{tabular}

Table 5.7: Tau kink candidate 3333_17665.

Track Information for Tau Candidate 3333_17665

\begin{tabular}{|c|c|c|c|c|}
\hline 3333_17665 & \multicolumn{2}{|c|}{ Momentum $(\mathrm{GeV})$} & \multicolumn{2}{|c|}{ Identification } \\
\hline Track & MS & Spec & Particle & \\
\hline 3 & & & tau & parent \\
4 & $21.4_{-6.4}^{+14}$ & $38 \pm 10$ & electron & daughter \\
5 & $1.7_{-0.4}^{+0.8}$ & & hadron & \\
6 & $3.9_{-1.3}^{+3.2}$ & & hadron & \\
7 & & & hadron & \\
8 & $3.9_{-0.8}^{+1.6}$ & & hadron & \\
9 & $3.5_{-0.9}^{+1.6}$ & & hadron & \\
\hline
\end{tabular}

Table 5.8: Tau candidate 3333_17665 has six charged primary tracks. The candidate tau track, track 3, decayed to an electron, track 4. This electron was identified in the emulsion. The daughter track traversed the EMCAL which provided a spectrometer momentum measurement.

\section{Tau Candidate 3333_17665}

In tau candidate 3333_17665, a neutrino interacted in the second emulsion module and produced six charged primary tracks. The candidate tau track, track 3 , decayed to an electron, track 4 . This electron was identified in the emulsion. The parameters of this event are summarized in Table 5.7. Multiple scattering momentum measurements were available for all but one track, and track 4 also had a spectrometer momentum measurement; these measurements are listed in Table 5.8. Fig. 5.14, Fig. 5.15, and Fig. 5.16 show the X-view, $\mathrm{U}$-view, and $\mathrm{V}$-view of the spectrometer. As Fig. 5.14 shows, there is a shower in the scintillating fibers, activity in the drift chamber, and activity in the EMCAL. This event has a total of $9.9 \pm 1.3 \mathrm{GeV}$ as measured in the EMCAL. There is a shower along track 4 , the daughter electron, in the scintillating fibers. Fig. 5.17 shows the emulsion data for this event. 


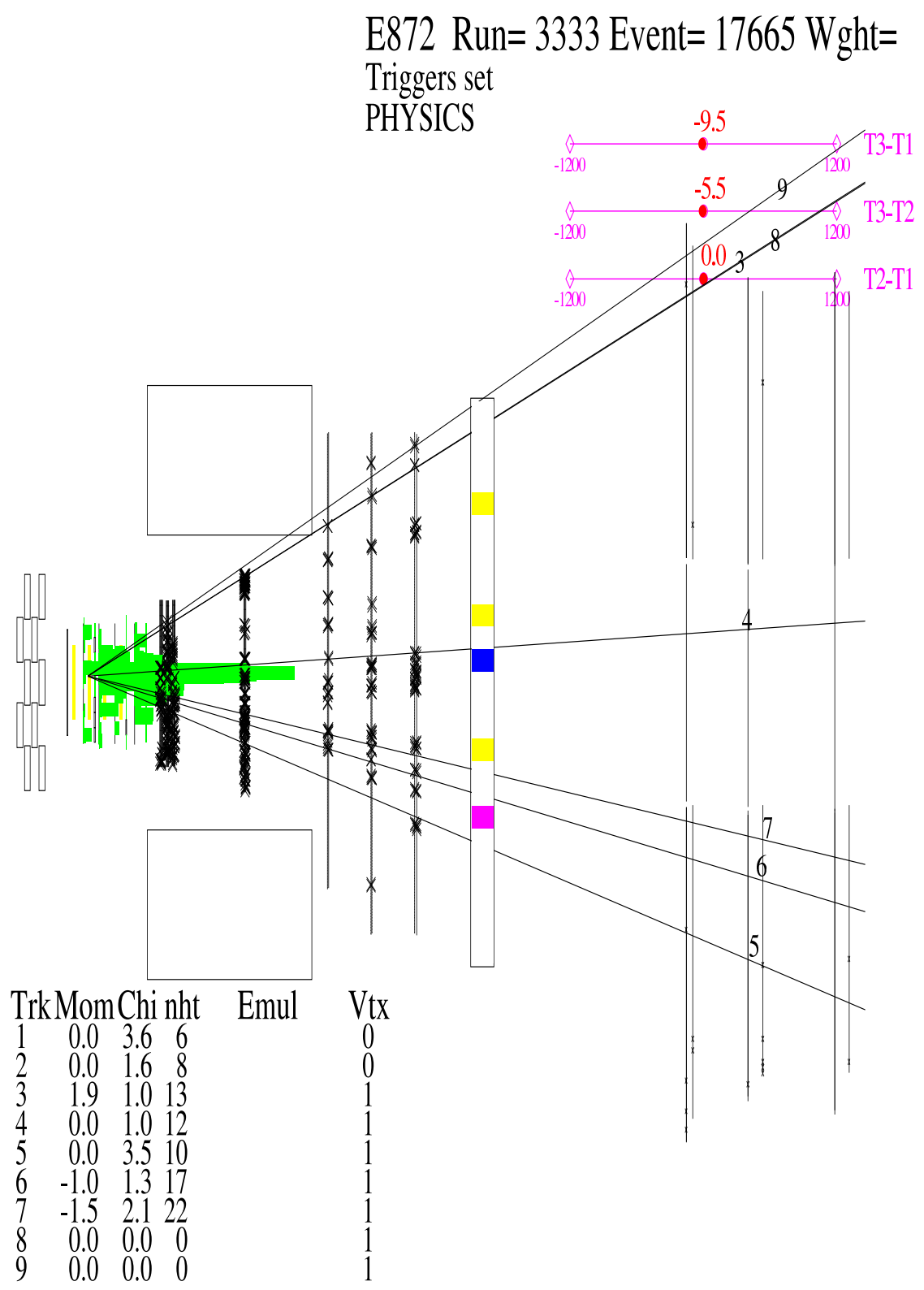

Figure 5.14: The X-view in the spectrometer of tau candidate 3333_17665. The tracks shown are the projections of the emulsion tracks onto the spectrometer. All tracks are charged hadrons, except track 3, which is the tau candidate, and track 4, which is the daughter electron. There is a shower in the scintillating fibers, activity in the drift chamber, and activity in the EMCAL. This event has a total of $9.9 \pm 1.3 \mathrm{GeV}$ deposited in the EMCAL. 


\section{E872 Run= 3333 Event $=17665$ Wght= 1.0 U View}
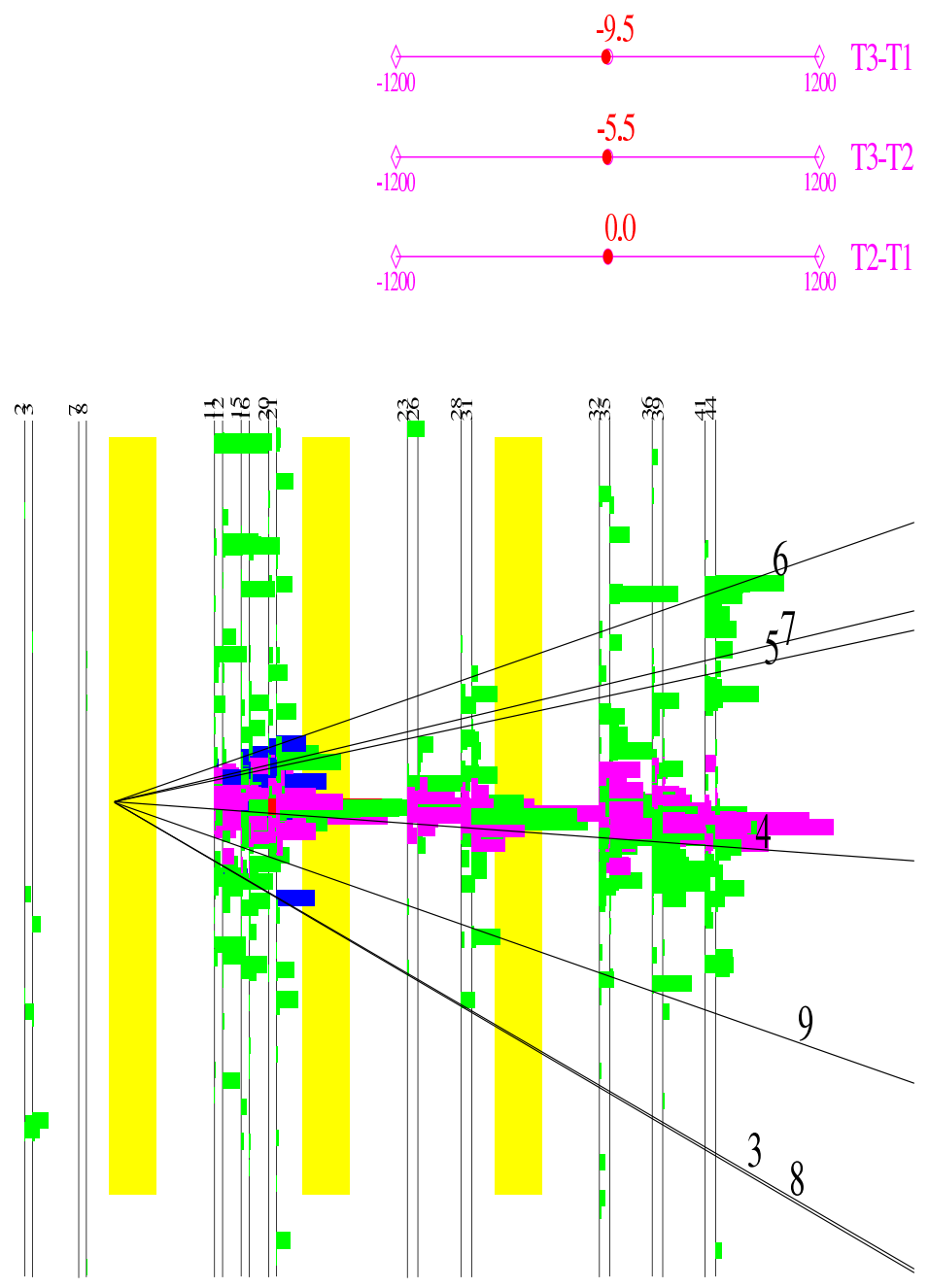

Figure 5.15: The U-view in the spectrometer of tau candidate 3333_17665. All tracks are charged hadrons, except track 3, which is the tau candidate, and track 4, which is the daughter electron. There is a shower along track 4, the daughter electron. 


\section{E872 Run= 3333 Event= 17665 Wght= 1.0 V View}
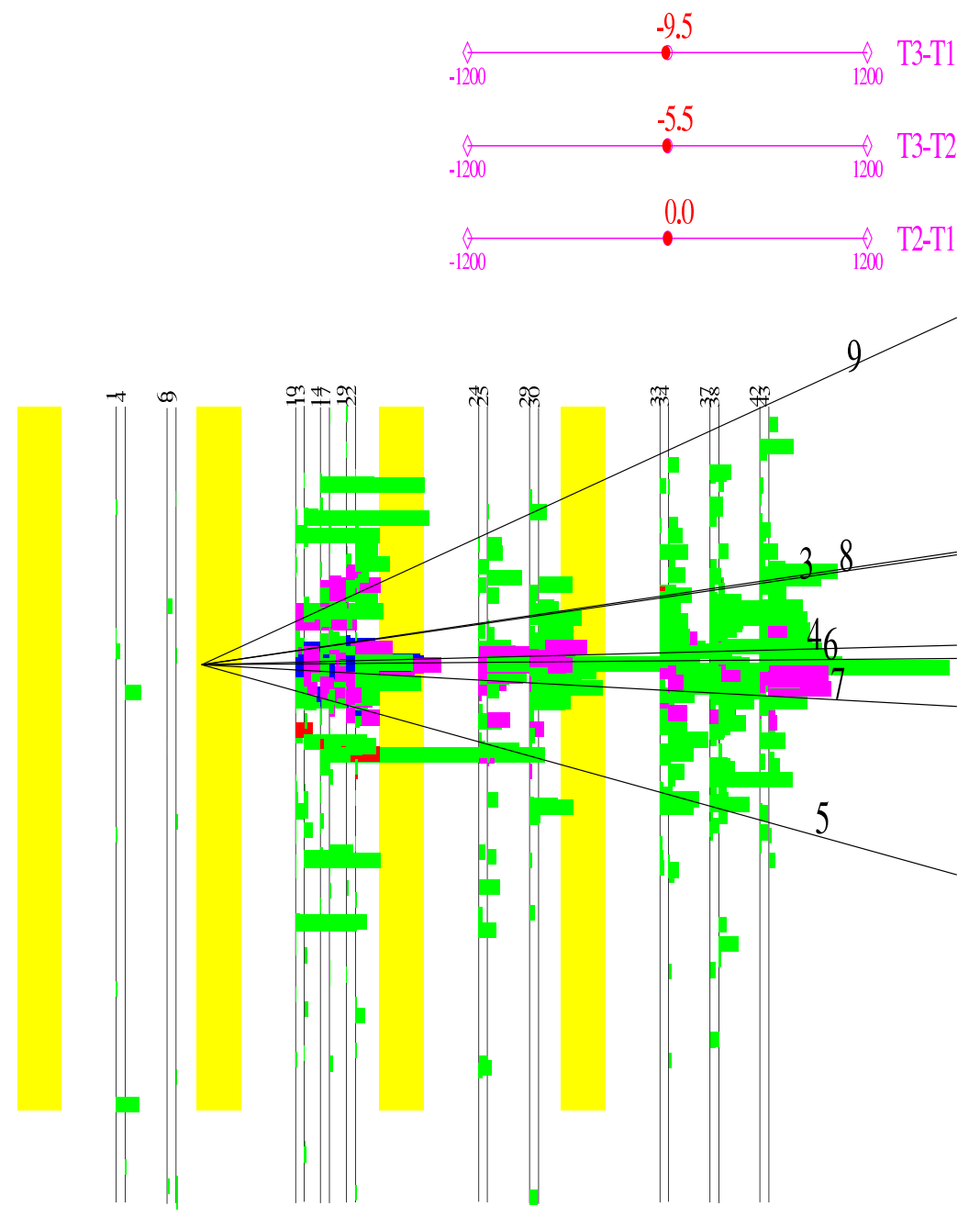

Figure 5.16: The V-view of the spectrometer data for tau candidate 3333_17665. 


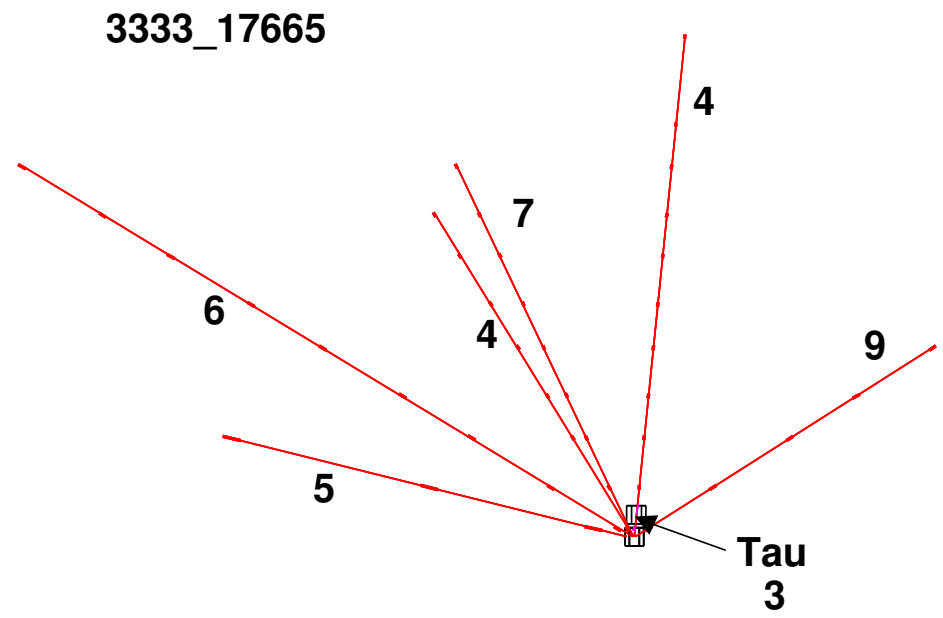

Figure 5.17: The emulsion data for tau candidate 3333_17665. All tracks are charged hadrons, except track 3, which is the tau candidate, and track 4, which is the daughter electron. The parameters and momentum measurements for this event are listed in Table 5.7 and 5.8. The neutrino interaction occurred in ECC800 type emulsion, and the neutrino interaction occurred in emulsion. 
Parameters of Tau Candidate 3140_22143

\begin{tabular}{|c|c|c|c|c|}
\hline$\theta(\mathrm{rad})$ & $\Delta \phi(\mathrm{rad})$ & $L(\mathrm{~mm})$ & $\alpha(\mathrm{rad})$ & Decay Material \\
\hline 0.040 & 1.67 & 4.8 & 0.012 & plastic \\
\hline
\end{tabular}

Table 5.9: Tau kink candidate 3140_22143.

The following three events did not appear in the original publication.

\section{Tau Candidate 3140_22143}

In tau candidate 3140_22143, a neutrino interacted in the fourth emulsion module and produced twelve charged primary tracks. The tau candidate, track 9 , decayed or interacted to produce a single charged hadron, track 1 . The parameters of this event are summarized in Table 5.9. Multiple scattering momentum measurements were available for all tracks, and tracks 1, 7, and 10 also had spectrometer momentum measurements, as shown in Table 5.10. Fig. 5.18, 5.19, and 5.20 show the $\mathrm{X}$-view, U-view, and V-view of the spectrometer. There is activity in the scintillating fibers, the drift chambers, and the EMCAL. The hadronic energy of this event is $51.6 \pm 5.9 \mathrm{GeV}$, as measured in the EMCAL. Fig. 5.19 and Fig. 5.20 show the scintillating fibers. Fig. 5.21 shows the emulsion data for this event. 
Track Information for Tau Candidate 3140_22143

\begin{tabular}{|c|c|c|c|c|}
\hline 3140_22143 & \multicolumn{2}{|c|}{ Momentum $(\mathrm{GeV})$} & \multicolumn{2}{|c|}{ Identification } \\
\hline Track & MS & Spec & Particle & \\
\hline 1 & $16.8_{-6.0}^{+18.4}$ & $22 \pm 5$ & hadron & daughter \\
2 & $6.6_{-1.6}^{+2.8}$ & & hadron & \\
3 & $3.0_{-0.6}^{+1.0}$ & & hadron & \\
4 & $4.8_{-1.0}^{+1.6}$ & & hadron & \\
5 & $6.1_{-1.5}^{+2.6}$ & & hadron & \\
6 & $19.4_{-6.3}^{+16}$ & & hadron & \\
7 & $5.8_{-1.3}^{+2.3}$ & $5 \pm 1$ & hadron & \\
8 & $0.3_{-0.0}^{+0.1}$ & & hadron & \\
9 & & & tau & parent \\
10 & $12.8_{-4.1}^{+10}$ & $26 \pm 7$ & hadron & \\
11 & $5.1_{-1.2}^{+2.6}$ & & hadron & \\
12 & $4.5_{-0.8}^{+1.3}$ & & hadron & \\
13 & $13.4_{-3.7}^{+7.0}$ & & hadron & \\
\hline
\end{tabular}

Table 5.10: Tau candidate 3140_22143 includes twelve charged primary tracks. The tau candidate, track 9, decayed or interacted to produce a single charged hadron, track 1 . Tracks 1, 7, and 10 traversed either the drift chambers or the EMCAL, which provided spectrometer momentum measurements. 


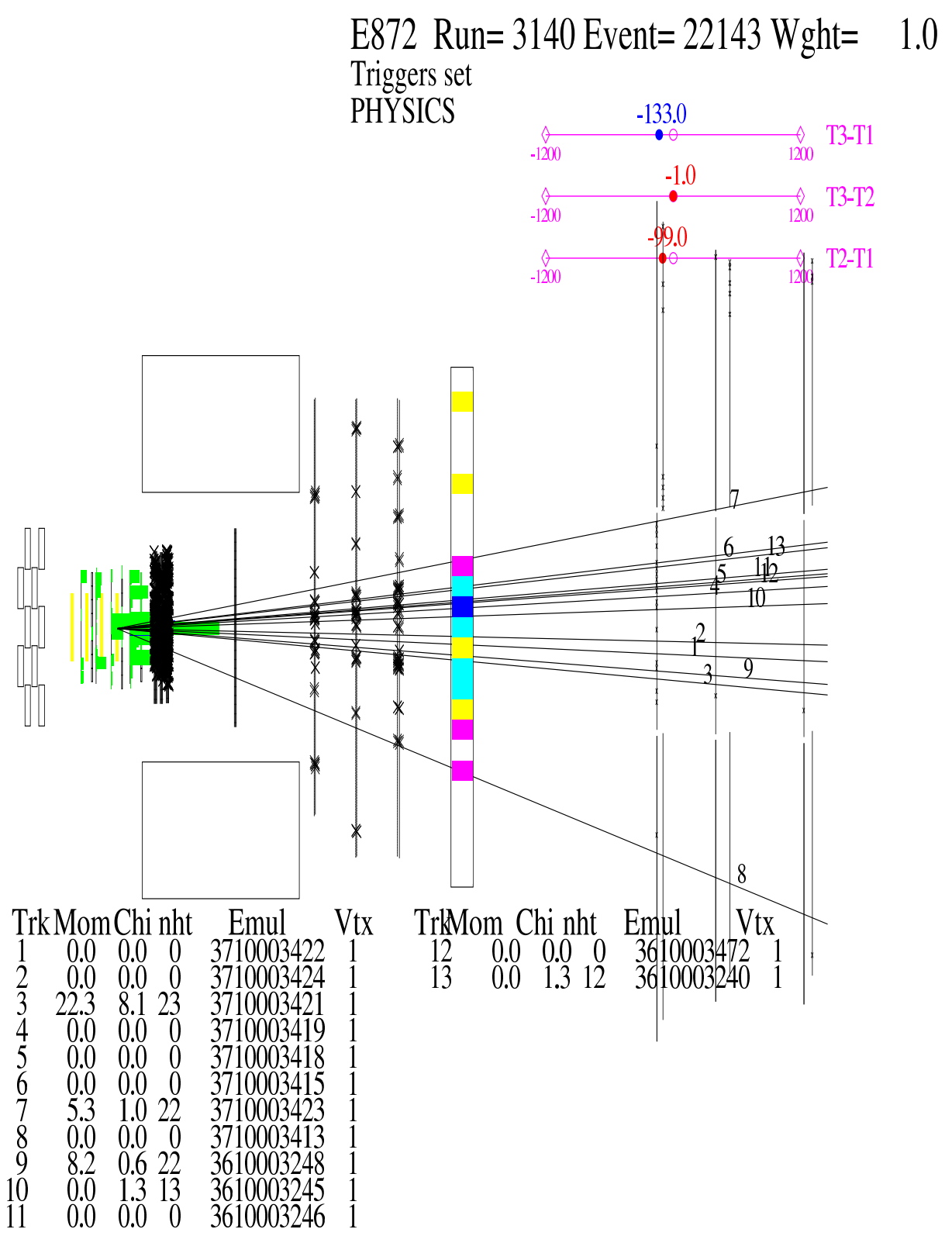

Figure 5.18: The X-view in the spectrometer for tau candidate 3140_22143. The tracks shown are the projections of the emulsion tracks onto the spectrometer. There are twelve charged primary tracks in this event. All tracks are charged hadrons except for track 9, which is the tau candidate. The tau candidate decayed to or interacted to produce track 1, a charged hadron. There is no showering in the scintillating fibers. Many of the tracks overlap, as there are many tracks in a small area. 


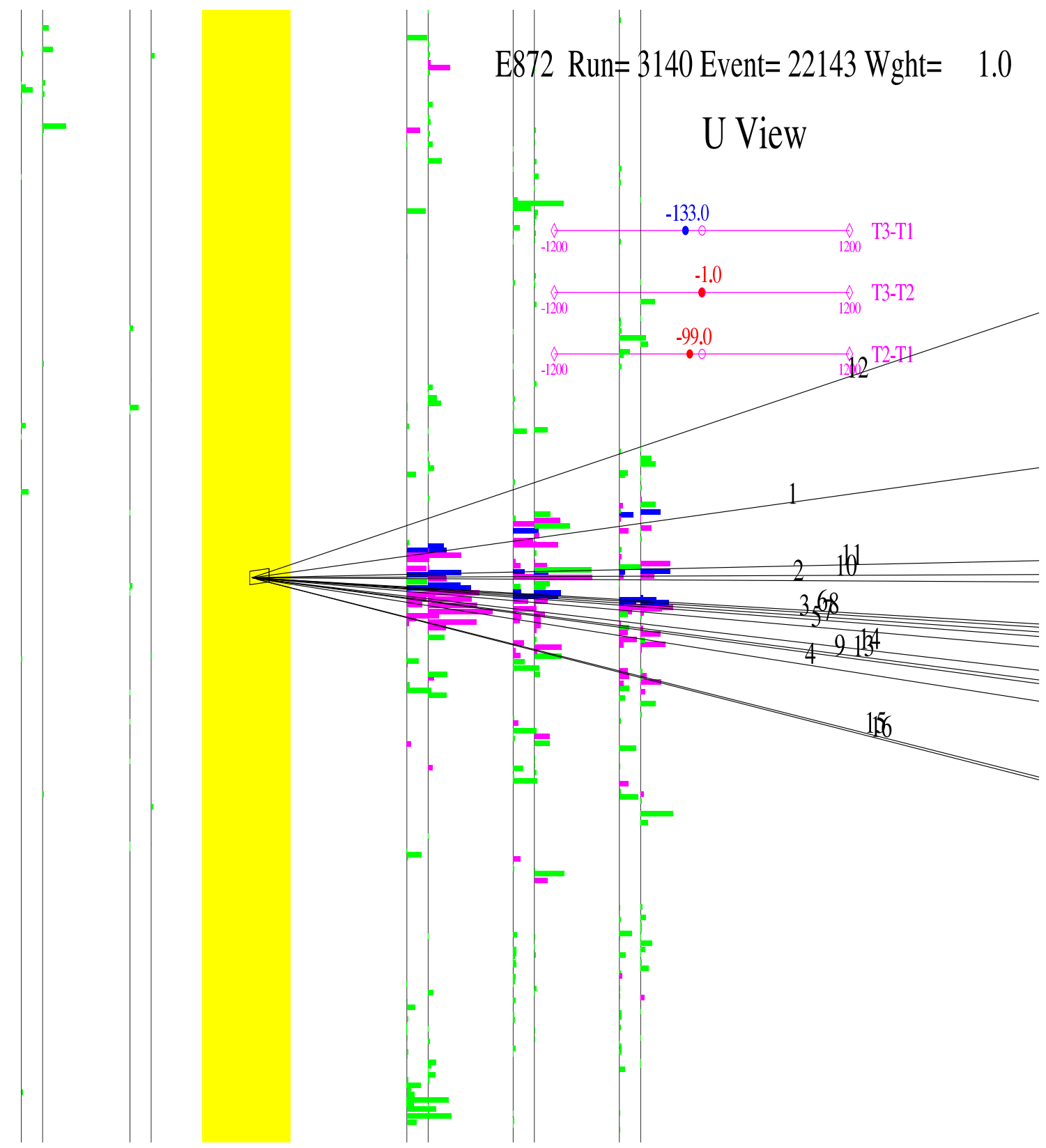

Figure 5.19: The U-view in the spectrometer for tau candidate 3140_22143. There are twelve charged primary tracks and one charged daughter track in this event. All tracks are charged hadrons except for track 9 , which is the tau candidate. The tau candidate decayed or interacted to produce to track 1, a charged hadron. 


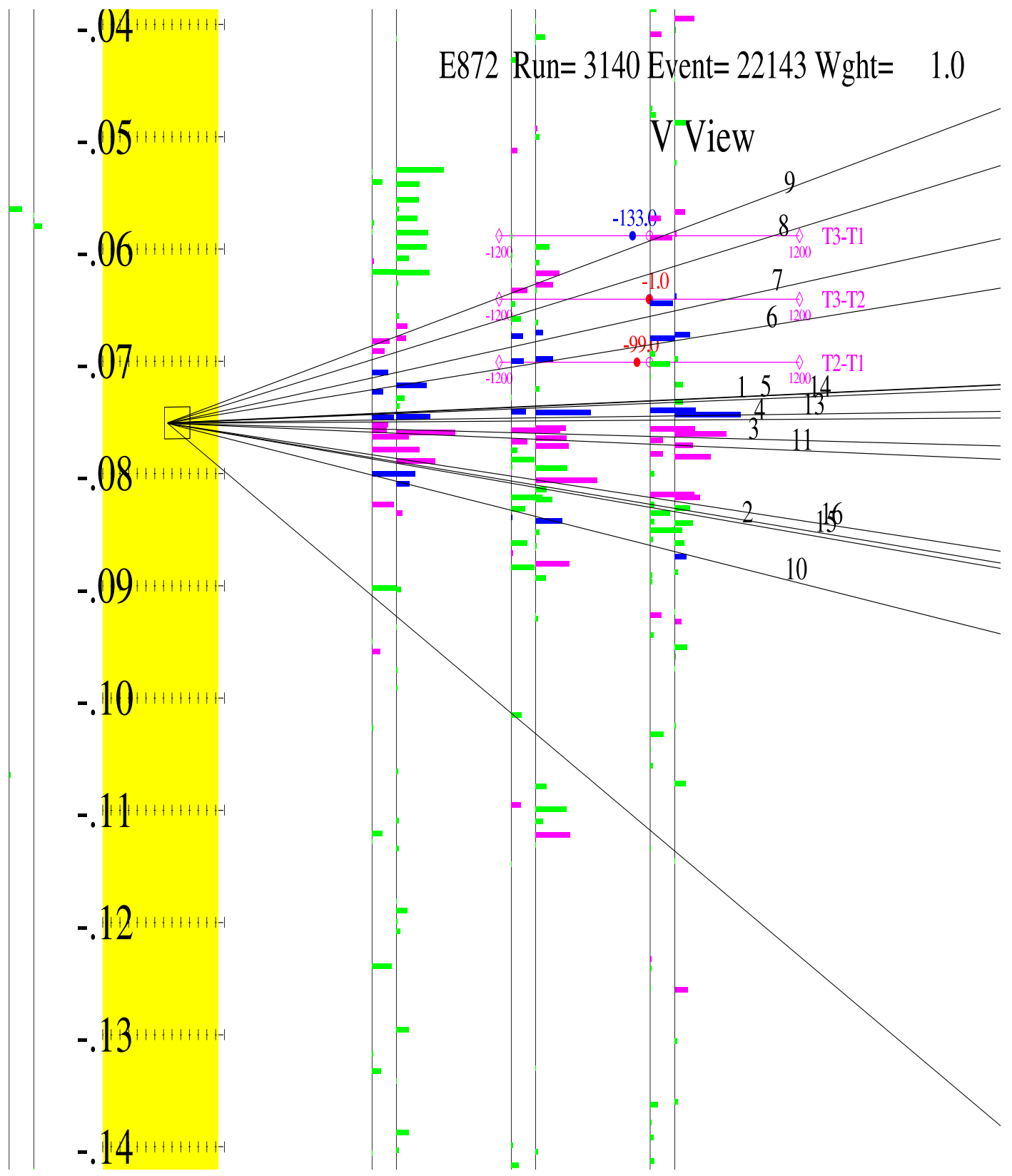

Figure 5.20: The V-view in the spectrometer for tau candidate 3140_22143. 


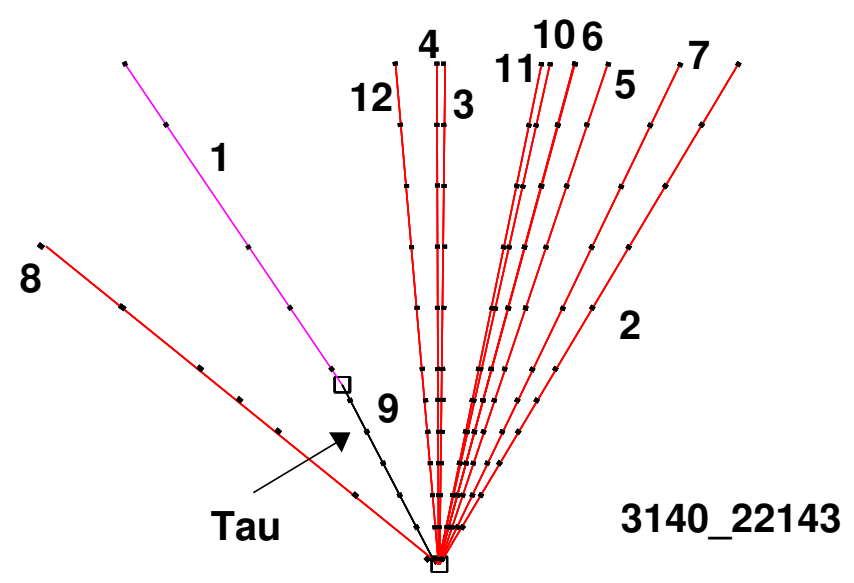

Figure 5.21: Emulsion data for tau candidate 3140_22143. All tracks are charged hadrons except for track 9 , which is the tau candidate. The tau candidate decayed to or interacted to produce track 1, a charged hadron. The parameters and momentum measurements for this event are listed in Table 5.9 and 5.10. The neutrino interaction occurred in ECC800, and the decay occurred in plastic. 
Parameters of Tau Candidate 3296_18816

\begin{tabular}{|c|c|c|c|c|}
\hline$\theta(\mathrm{rad})$ & $\Delta \phi(\mathrm{rad})$ & $L(\mathrm{~mm})$ & $\Sigma I P(\mathrm{rad})$ & Decay Material \\
\hline 0.141 & 1.74 & 0.78 & 0.029 & emulsion \\
\hline
\end{tabular}

Table 5.11: Tau trident candidate 3296_18816.

Track Information for Tau Candidate 3296_18816

\begin{tabular}{|c|c|c|c|c|}
\hline 3296_18816 & \multicolumn{2}{|c|}{ Momentum $(\mathrm{GeV})$} & \multicolumn{2}{|c|}{ Identification } \\
\hline Track & MS & Spec & Particle & \\
\hline 1b & $3.2_{-1.3}^{+5.2}$ & & hadron & \\
2b & $1.7_{-0.6}^{+1.9}$ & & hadron & \\
3b & $1.3_{-0.5}^{+1.4}$ & & hadron & \\
4b & $1.0_{-0.3}^{+0.6}$ & & hadron & \\
5b & $1.3_{-0.4}^{+0.7}$ & & hadron & \\
6b/4a & & & tau & parent \\
1a & $1.3_{-0.5}^{+2.1}$ & & hadron & daughter \\
2a & $2.3_{-0.6}^{+1.3}$ & & hadron & daughter \\
3a & $0.9_{-0.3}^{+1.0}$ & & hadron & daughter \\
\hline
\end{tabular}

Table 5.12: Tau trident candidate 3296_18816 includes six charged primary tracks. The tau candidate, track $6 \mathrm{~b} / 4 \mathrm{a}$, decayed to or interacted to produce three charged daughters, tracks 1a, 2a, and 3a. No tracks were unambiguously fit in the spectrometer; therefore, there were no spectrometer momentum measurements.

\section{Tau Candidate 3296_18816}

In tau candidate 3296_18816, a neutrino interacted in the second emulsion module and produced six charged primary tracks. Two sets of spectrometer data are included for this event. The first set shows the primary tracks, which are labeled with a "b". The second set shows the parent track and the secondary tracks, which are labeled with an "a". The tau candidate, track $6 \mathrm{~b} / 4 \mathrm{a}$, decayed to or interacted to produce three charged daughters, 1a, 2a, and 3a. The parameters of this event are summarized in Table 5.11. Only multiple scattering momentum measurements were available and are listed in Table 5.10. Fig. 5.22, Fig. 5.23, and Fig. 5.24 show the X-view, U-view, and V-view of the spectrometer with projections of tracks from the primary vertex. Fig. 5.25, Fig. 5.26, and Fig. 5.27 show the $\mathrm{X}$-view, U-view, and $\mathrm{V}$-view of the spectrometer with projections of tracks from the secondary vertex. Fig. 5.28 shows the emulsion data for this event. 


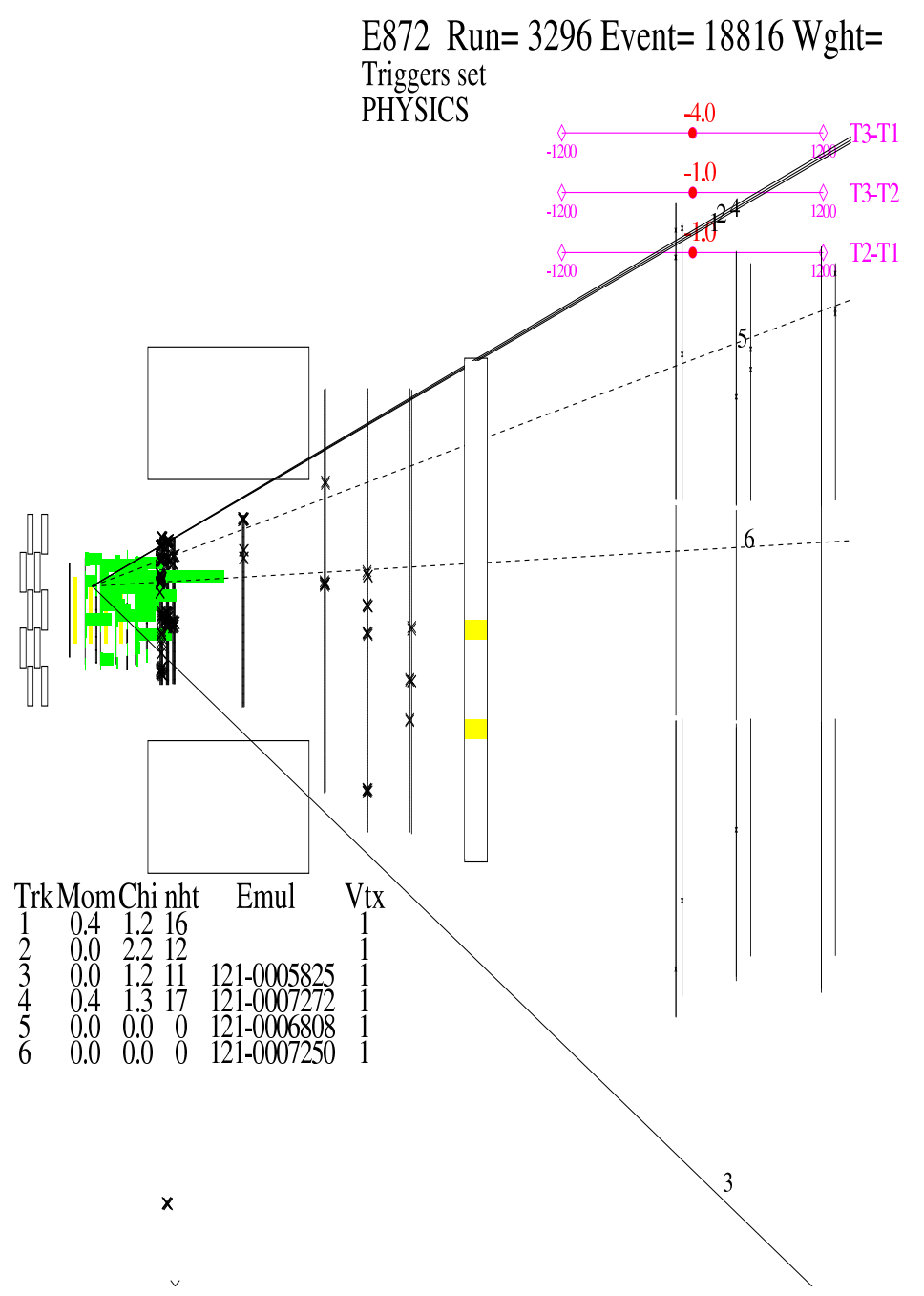

Figure 5.22: The X-view in the spectrometer of tau candidate 3296_18816 with the projections of the tracks from the primary vertex. The tracks shown are the projections of the emulsion tracks onto the spectrometer. All tracks are hadrons, except track 6, which is the tau candidate. There is activity in the scintillating fibers. Tracks 2 and 4 overlap in this view, but not in the others. 


\section{E872 Run= 3296 Event $=18816$ Wght= 1.0 View}
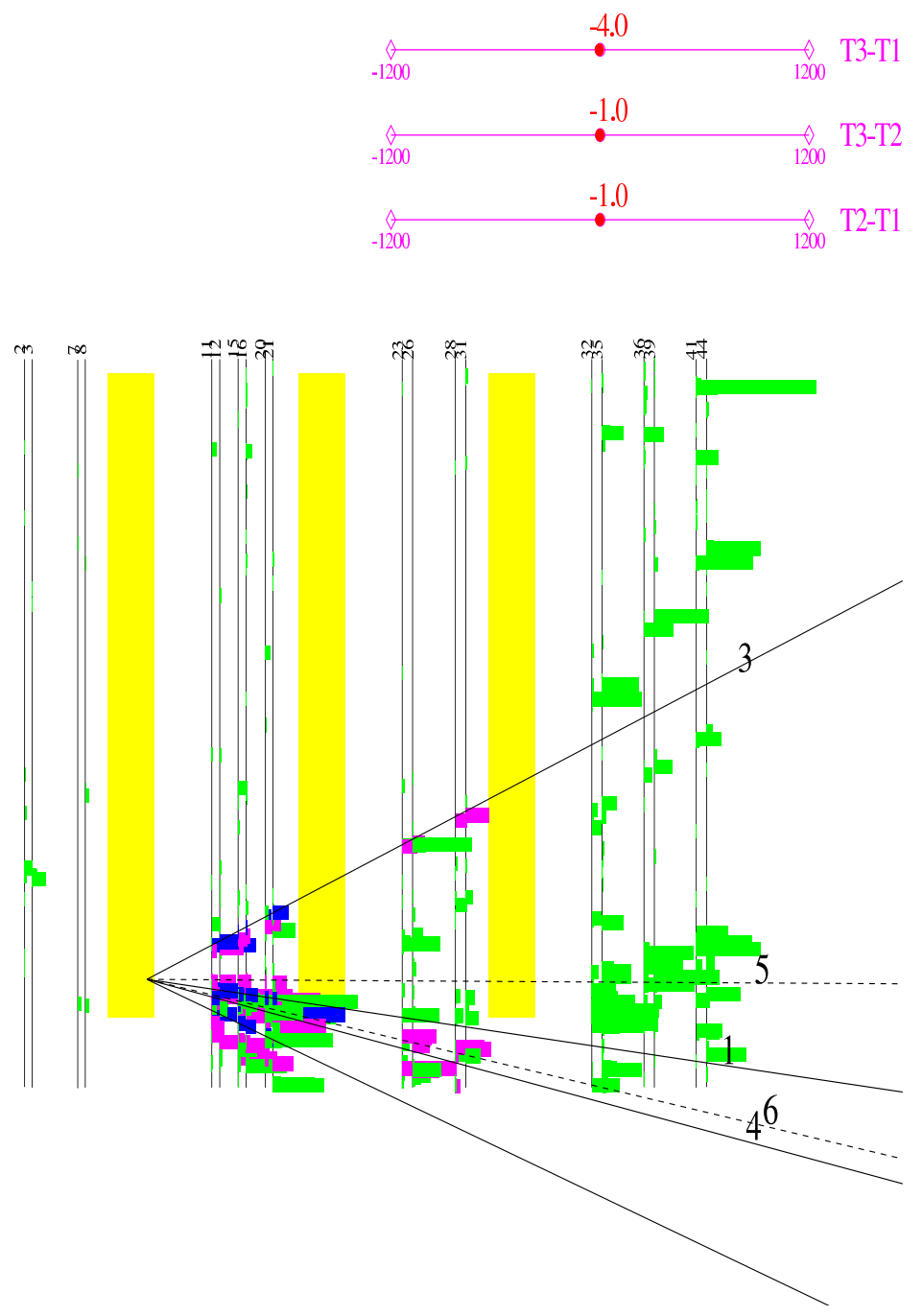

Figure 5.23: The U-view in the spectrometer of tau candidate 3296_18816 with the projections of the tracks from the primary the primary vertex. All tracks are hadrons, except track 6 , which is the tau candidate. Track 2, which is the bottom track, is labeled to the left of the figure. 


\section{E872 Run= 3296 Event= 18816 Wght= 1.0 $\mathrm{V}$ View}
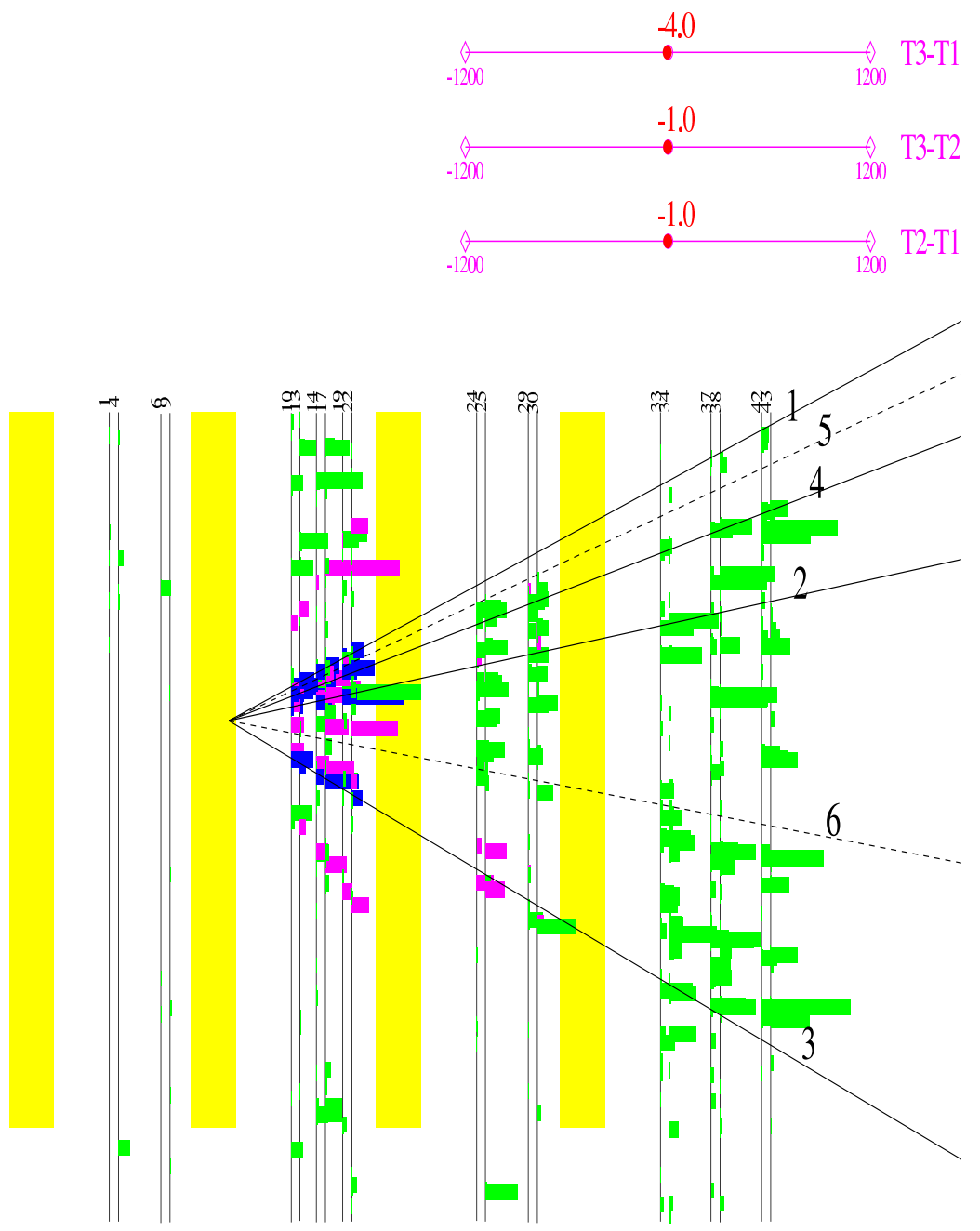

Figure 5.24: The V-view in the spectrometer of tau candidate 3296_18816 with the projections of the tracks from the the primary vertex. 


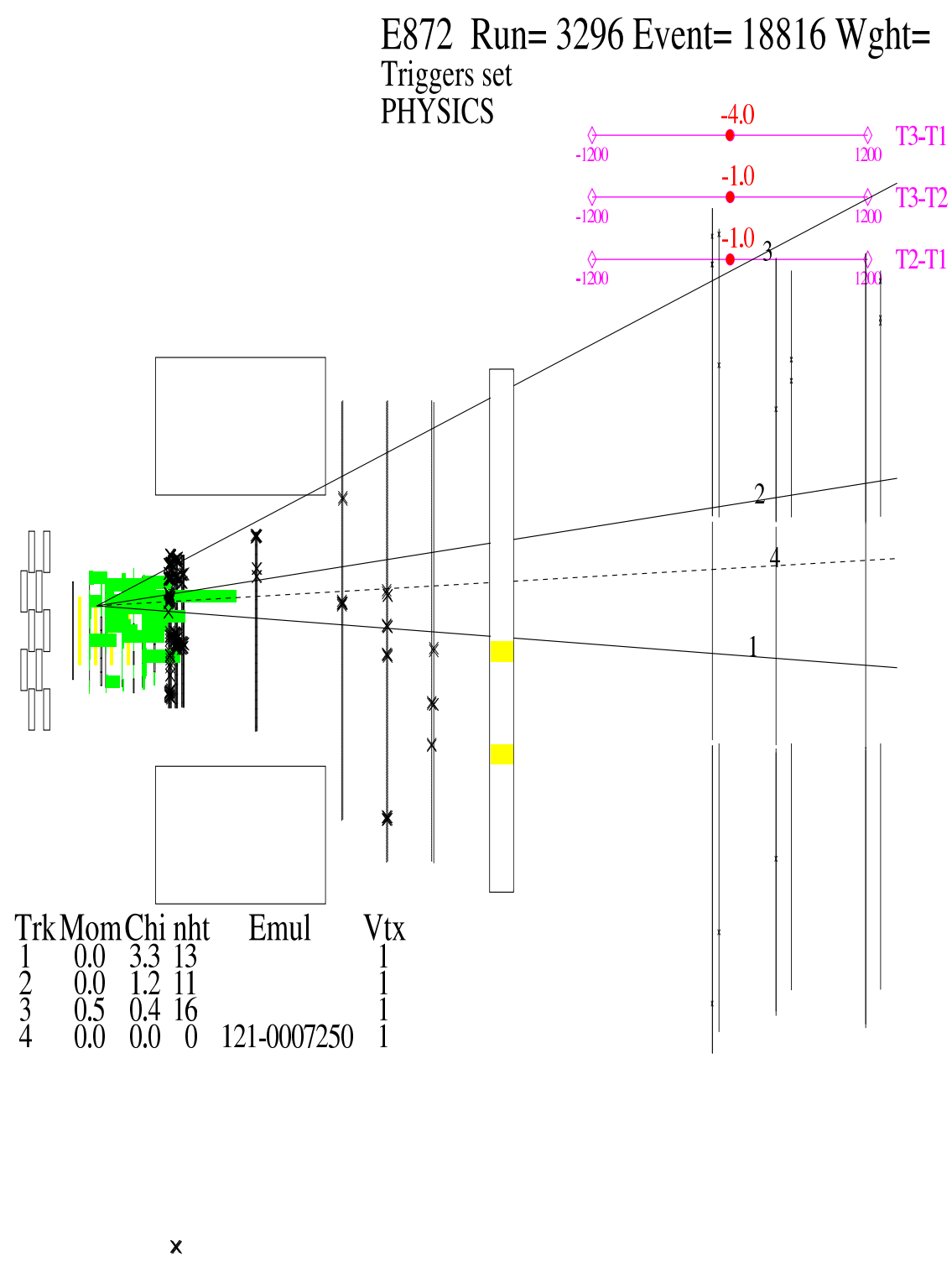

Figure 5.25: The X-view in the spectrometer of tau candidate 3296_18816 with the projections of the tracks from the secondary vertex. Track 4 is the tau candidate. Tracks 1 , 2 , and 3 are the charged hadron daughters of the candidate tau decay. 


\section{E872 Run= 3296 Event $=18816$ Wght= 1.0 U View}
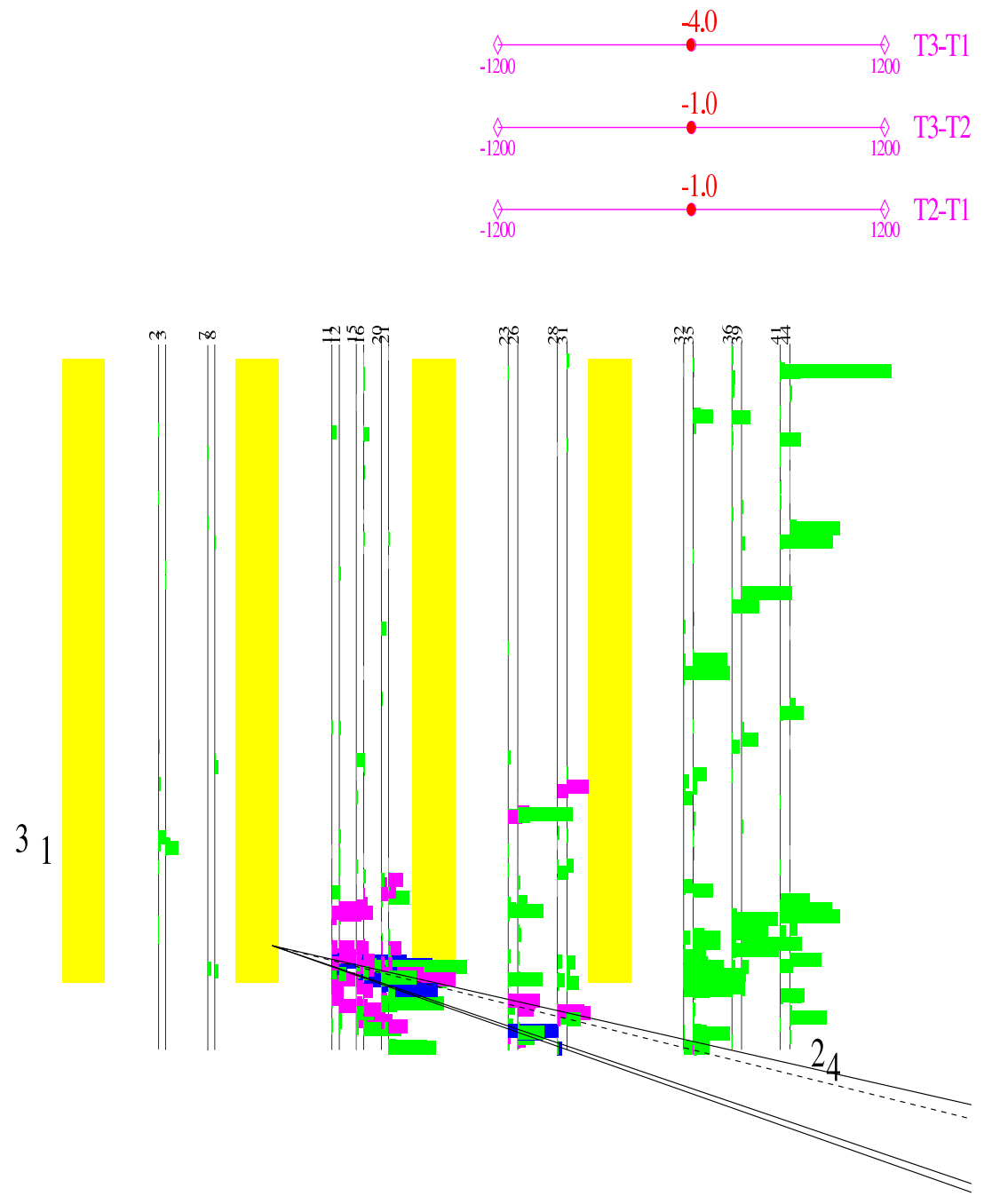

Figure 5.26: The U-view in the spectrometer of tau candidate 3296_18816 with the projections of the tracks from the secondary vertex. Track 4 is the tau candidate. Tracks 1, 2, and 3 are the charged hadron daughters of the candidate tau decay. The labels for tracks 1 and 3 are to the left of the figure. Track 3 is the lowest track, and track 1 is directly above track 3 . 


\section{E872 Run= 3296 Event= 18816 Wght= 1.0 V View}
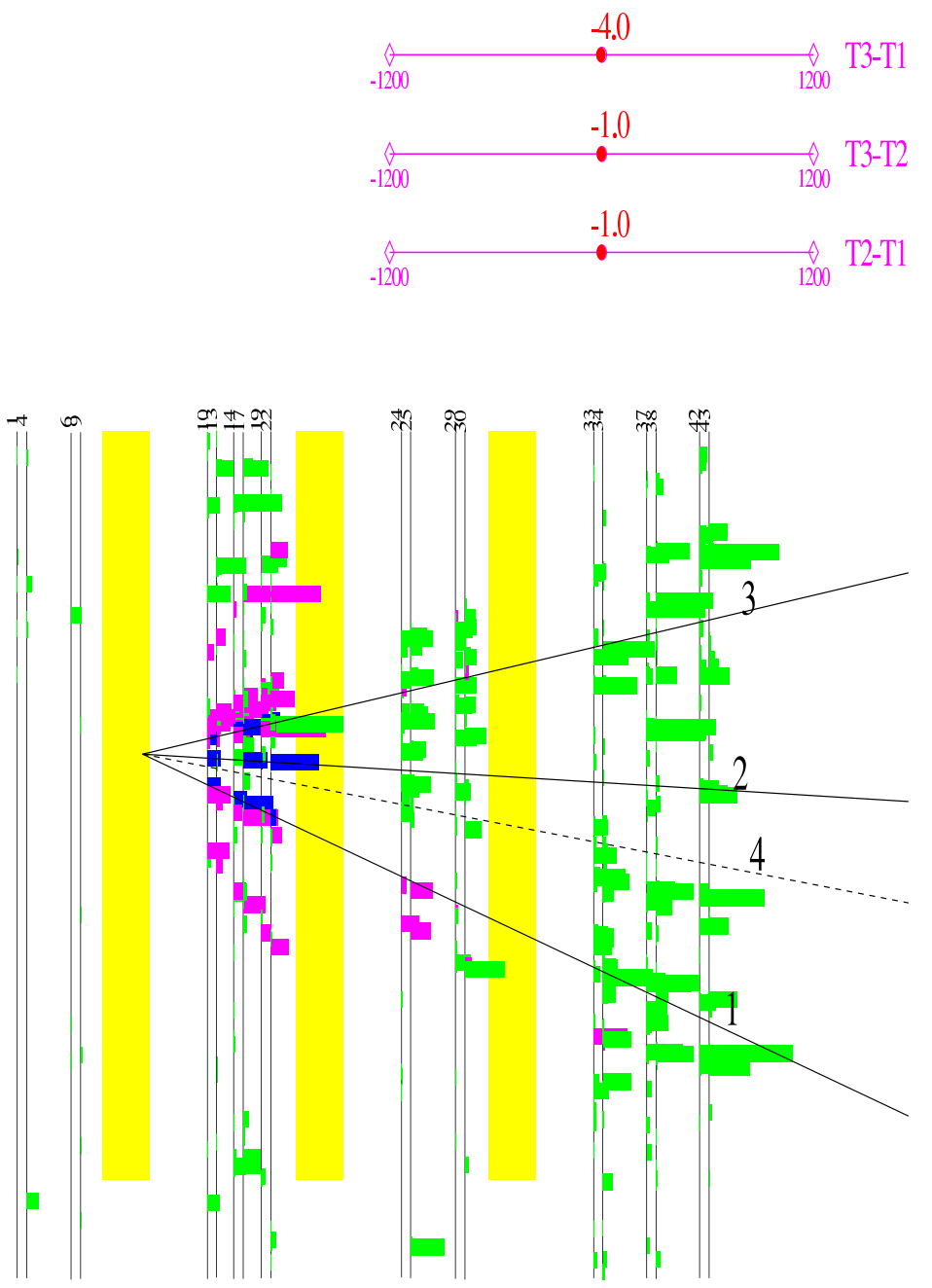

Figure 5.27: The V-view in the spectrometer of tau candidate 3296_18816 with the projections of tracks from the secondary vertex. 


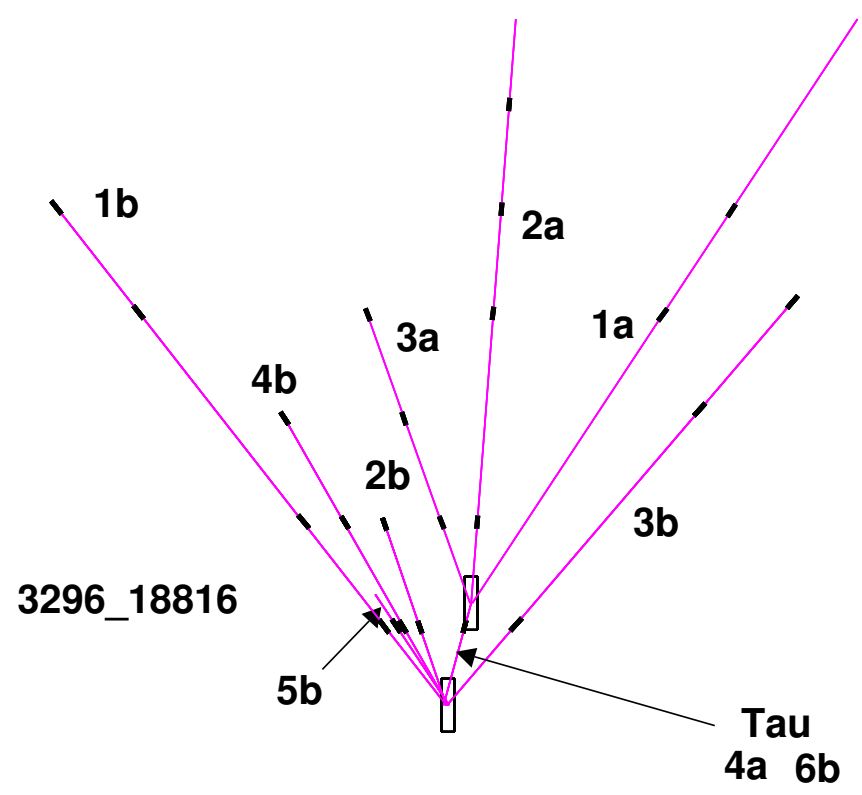

Figure 5.28: The emulsion data for tau candidate 3296_18816. The primary tracks shown in the first set of spectrometer figures are designated with a "b" at the end of the number, and the tracks from the second set of spectrometer figures, which are the parent and daughter tracks, are designated with an "a". All tracks, except track 4a/6b, are charged hadrons. The parameters and momentum measurements for this event are listed in Table 5.11 and 5.12. The neutrino interaction occurred in ECC200 type emulsion, and the decay occurred in the emulsion. 
Parameters of Tau Candidate 3334_19920

\begin{tabular}{|c|c|c|c|c|}
\hline$\theta(\mathrm{rad})$ & $\Delta \phi(\mathrm{rad})$ & $L(\mathrm{~mm})$ & $\Sigma I P(\mathrm{~mm})$ & Decay Material \\
\hline 0.040 & 3.11 & 7.8 & 0.036 & plastic \\
\hline
\end{tabular}

Table 5.13: Tau trident candidate 3334_19920.

\section{Tau Candidate 3334_19920}

In tau candidate 3334_19920, a neutrino interacted in the second emulsion module and produced nine charged primary tracks. One is a tau candidate, track 2 , that decayed or interacted to produce three charged particles, tracks 10, 11, and 12 . The parameters of this event are summarized in Table 5.13. Multiple scattering momentum measurements were available for all tracks, and track 8 also had a spectrometer momentum measurement, as shown in Table 5.14. Fig. 5.29, Fig. 5.30, and Fig. 5.31 show the X-view, U-view, and $\mathrm{V}$-view of the spectrometer. As Fig. 5.29 shows, there is showering in the scintillating fibers, activity in the drift chambers, and activity in the EMCAL. The hadronic energy in this event is $53.7 \pm 6.1 \mathrm{GeV}$. Fig. 5.30 and Fig. 5.31 show the scintillating fiber data for this event. There is a large hadronic shower which developed in the scintillating fibers. Fig. 5.32 shows the emulsion data for this event. 
Track Information for Tau Candidate 3334_19920

\begin{tabular}{|c|c|c|c|c|}
\hline 3334_19920 & \multicolumn{2}{|c|}{ Momentum $(\mathrm{GeV})$} & \multicolumn{2}{|c|}{ Identification } \\
\hline Track & MS & Spec & Particle & \\
\hline 1 & $1.3_{-0.2}^{+0.3}$ & & hadron & \\
2 & & & tau & parent \\
3 & $0.3_{-0.1}^{+0.1}$ & & hadron & \\
4 & $15_{-5.7}^{+17}$ & & hadron & \\
5 & $1.0_{-0.2}^{+0.3}$ & & hadron & \\
6 & $5.1_{-1.1}^{+1.8}$ & & hadron & \\
7 & $19.4_{-20}^{+32}$ & & hadron & \\
8 & $22.2_{-9.1}^{+37}$ & $59 \pm 15$ & hadron & \\
9 & $3.8_{-1.0}^{+1.8}$ & & hadron & \\
10 & $18.9_{-6.8}^{+21}$ & & hadron & daughter \\
11 & $3.3_{-0.7}^{+1.2}$ & & hadron & daughter \\
12 & $7.8_{-2.0}^{+3.6}$ & & hadron & daughter \\
\hline
\end{tabular}

Table 5.14: Tau trident candidate 3334_19920 includes nine charged primary tracks, one of which was a tau candidate, track 2 , that decayed or interacted to produce into three charged particles, track 10, 11, and 12. Track 8 was fit in the drift chambers, which provided a spectrometer momentum measurement. 


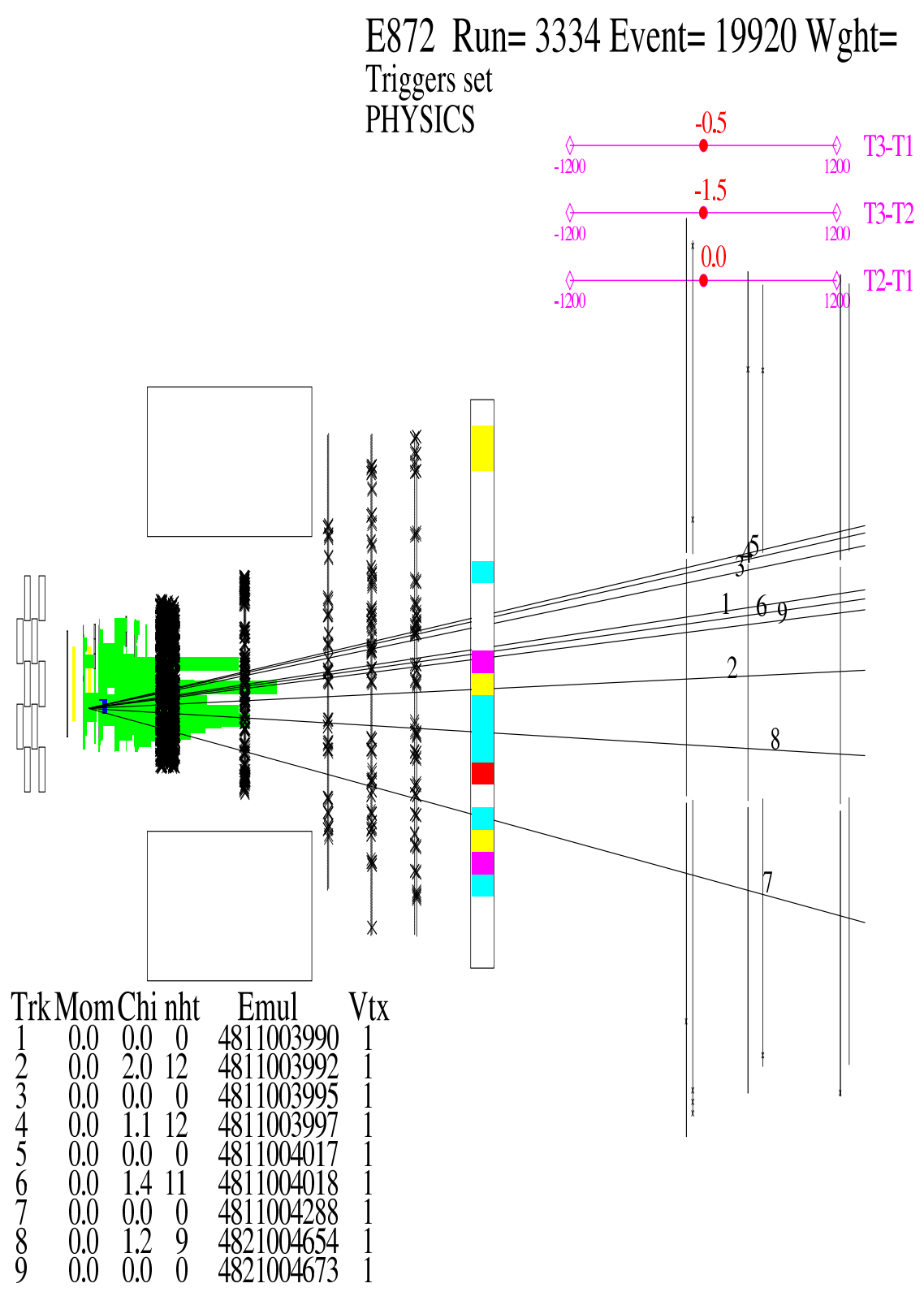

Figure 5.29: The X-view in the spectrometer data for tau candidate 3334_19920. The tracks shown are the projections of the emulsion tracks onto the spectrometer. All tracks are hadrons, except track 2, the tau candidate, which decayed to or interacted to produce three charged hadrons. There is showering in the scintillating fibers, activity in the drift chambers, and activity in the EMCAL. The hadronic energy in this event is $53.7 \pm 6.1$ $\mathrm{GeV}$. 


\section{E872 Run= 3334 Event= 19920 Wght= 1.0 U View}
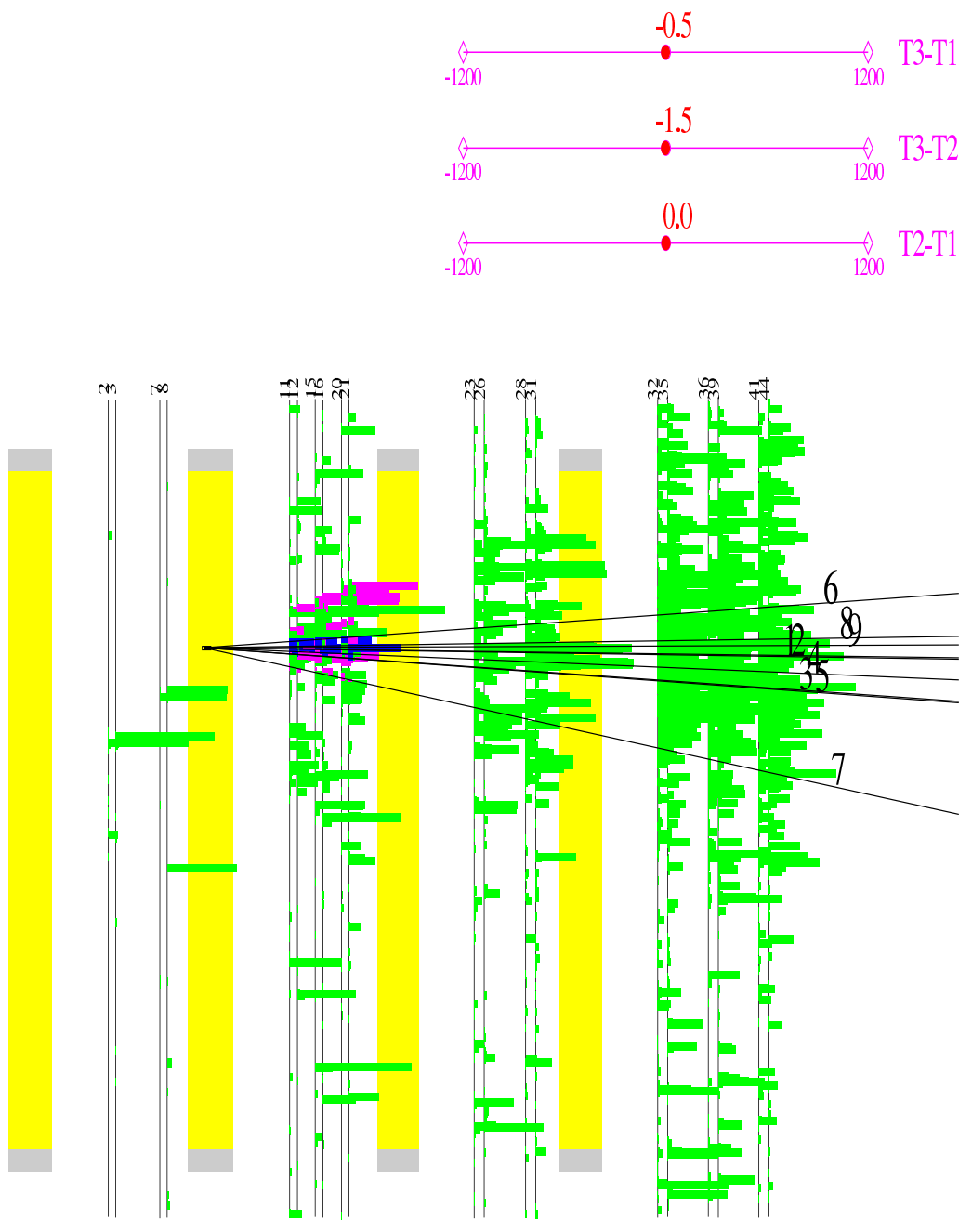

Figure 5.30: The U-view of the spectrometer data for tau candidate 3334_19920. All tracks are hadrons, except track 2, the tau candidate, which decayed to or interacted to produce three charged hadrons. There is a large hadronic shower which developed in the scintillating fibers. 
103

\section{E872 Run= 3334 Event= 19920 Wght= 1.0 V View}
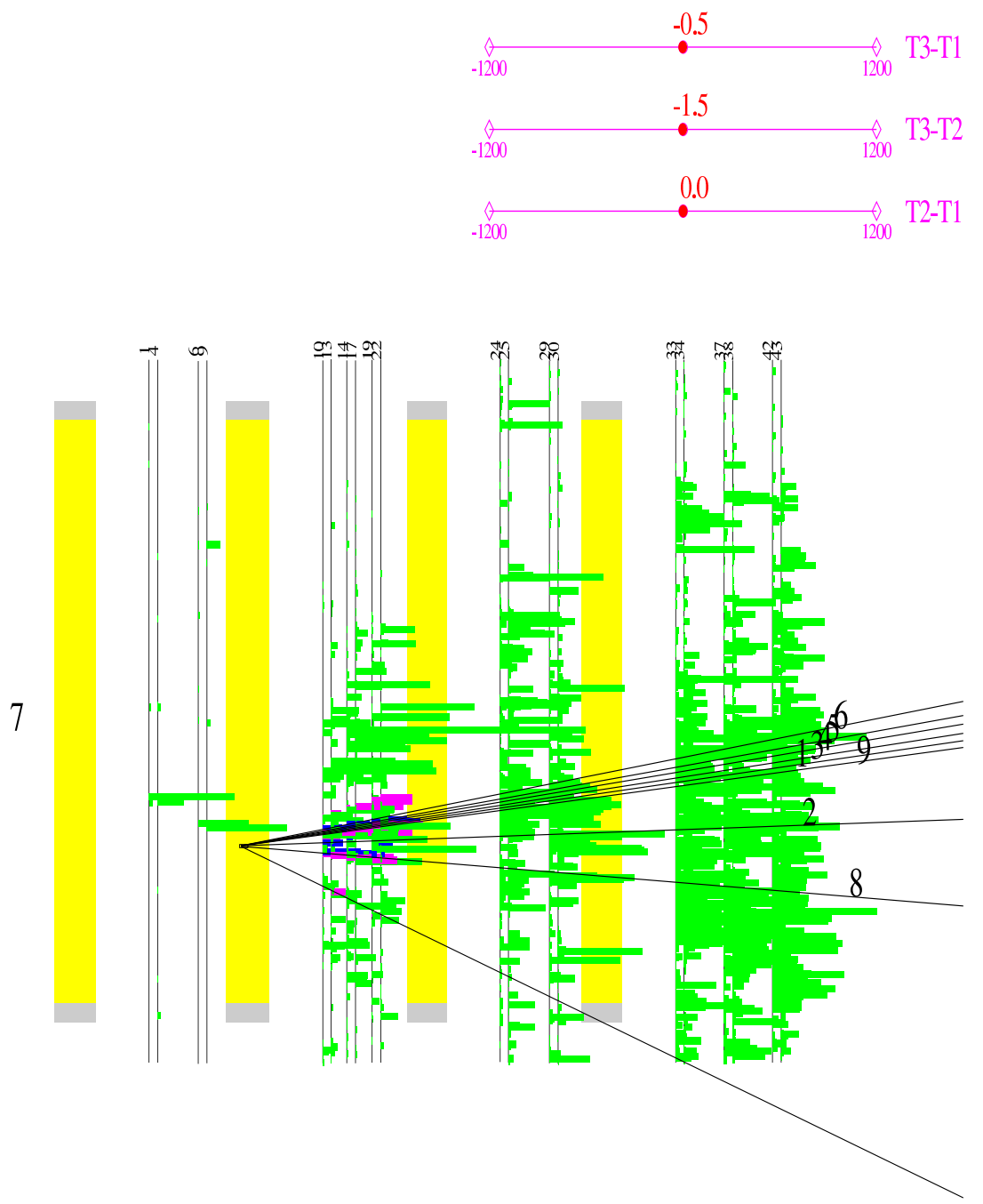

Figure 5.31: The V-view for the spectrometer data for tau candidate 3334_19920. The lowest track is track 7, which is labeled to the left of the figure. 


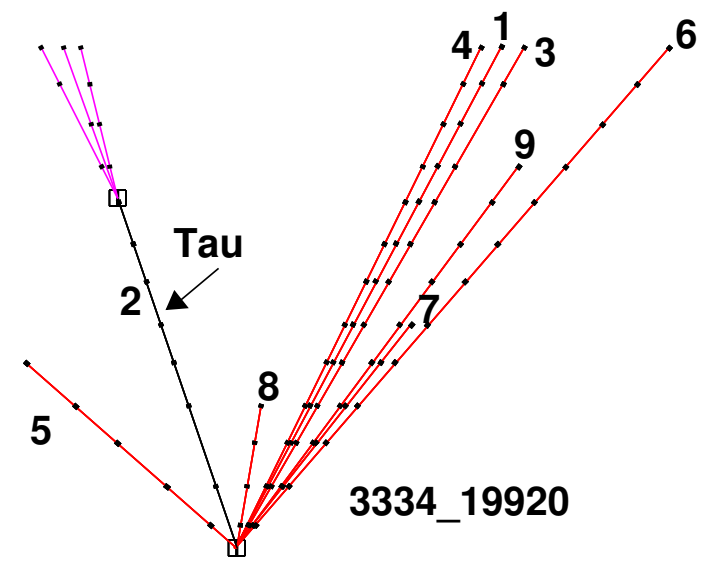

Figure 5.32: The emulsion data for tau candidate 3334_19920. All tracks are hadrons, except track 2 , the tau candidate, which decayed to or interacted to produce three charged hadrons. The parameters and momentum measurements for this event are listed in Table 5.13 and 5.14. The neutrino interacted in ECC800 type emulsion, and the decay occurred in plastic. 
Parameters of Charm Candidate 2846_09042

\begin{tabular}{|c|c|c|c|c|}
\hline$\theta(\mathrm{rad})$ & $\Delta \phi(\mathrm{rad})$ & $L(\mathrm{~mm})$ & $\alpha(\mathrm{rad})$ & Decay Material \\
\hline 0.034 & 2.81 & 7.0 & 0.165 & steel \\
\hline
\end{tabular}

Table 5.15: Charm kink candidate 2846_09042.

Track Information for Charm Candidate 2846_09042

\begin{tabular}{|c|c|c|c|c|}
\hline 2846_09042 & \multicolumn{2}{|c|}{ Momentum (GeV) } & \multicolumn{2}{|c|}{ Identification } \\
\hline Track & MS & Spec & Particle & \\
\hline 1 & & & charm & parent \\
2 & $16_{-4.0}^{+16}$ & $26 \pm 4$ & muon & \\
3 & $9.0_{-2.0}^{+5.0}$ & & hadron & \\
4 & $1.8_{-0.4}^{+0.7}$ & & hadron & \\
5 & $1.3_{-0.3}^{+0.6}$ & & hadron & \\
6 & $26_{-9.0}^{+21}$ & $58 \pm 23$ & electron & daughter \\
\hline
\end{tabular}

Table 5.16: Charm candidate 2846_09042 includes five charged primary tracks. One of the primary tracks is a charm candidate, track 1, which decayed to an electron, track 6 , which was as identified using pair production in the emulsion. Another primary track, track 2 , is a muon, which was identified in the muon ID. Two tracks, 2 and 6 , were fit in the spectrometer which provided spectrometer momentum measurements.

\subsection{Charm Candidates}

\section{Charm Candidate 2846_09042}

In charm candidate 2846_09042, a neutrino interacted in the second module and produced five charged primary tracks. One of the tracks is a charm candidate, track 1 , which decayed to an electron, track 6 , identified using pair production in the emulsion. Another primary track, track 2, is a muon, which was identified in the muon ID. The parameters of this event are summarized in Table 5.15. Multiple scattering measurements were available for all tracks, and tracks 2 and 6 also have spectrometer momentum measurements; these are listed in Table 5.16. Fig. 5.33, Fig. 5.34, and Fig. 5.35 show the X-view, U-view, and $\mathrm{V}$-view of the spectrometer. As Fig. 5.33 shows, there is showering in the scintillating fibers and activity in the drift chambers, EMCAL, and muon ID. The electromagnetic energy of this event is $49.2 \pm 5.6 \mathrm{GeV}$, as measured by the EMCAL. Fig. 5.36 shows the emulsion data for this event. 


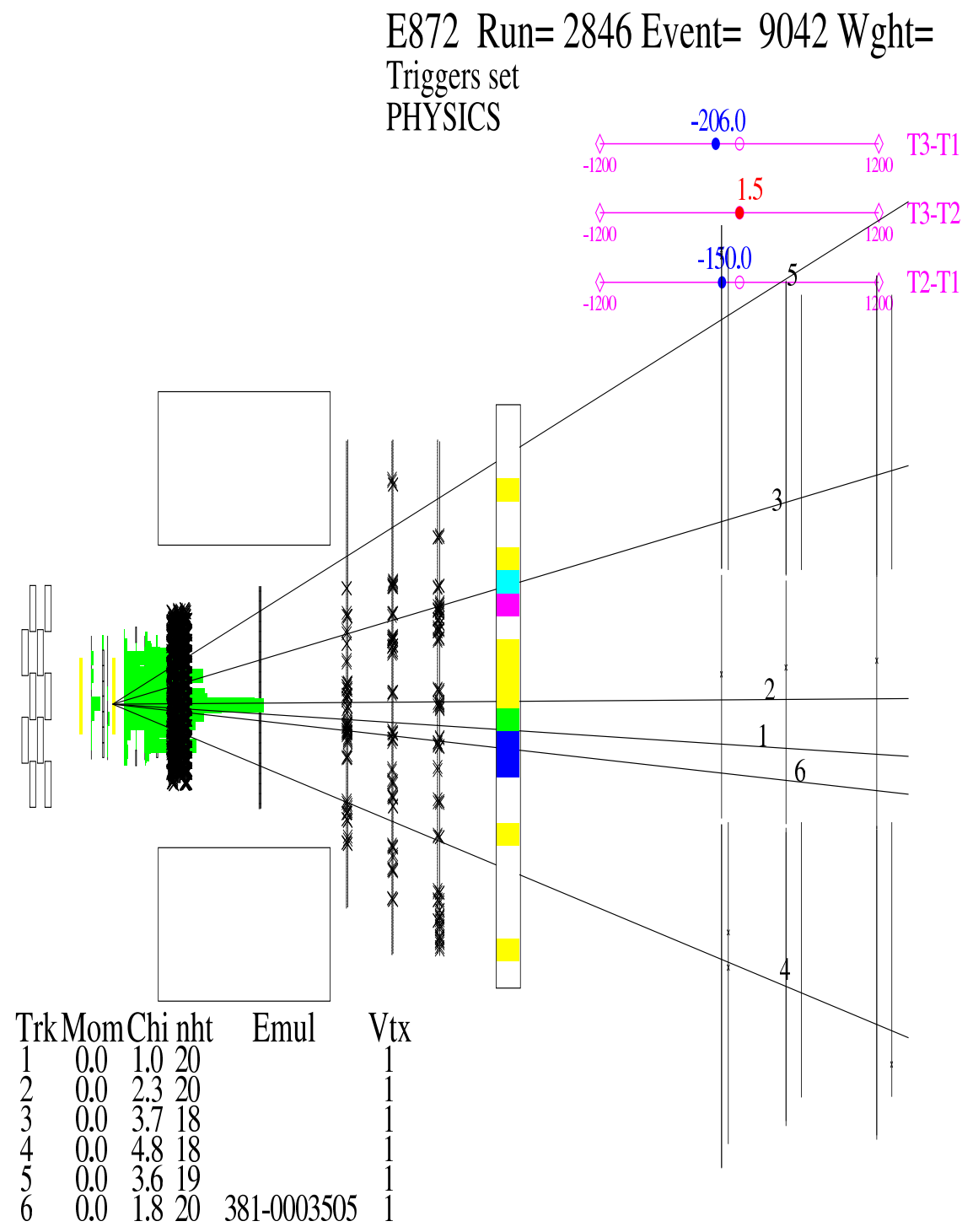

Figure 5.33: The X-view in the spectrometer for charm candidate 2846_09042. The tracks shown are the projections of the emulsion tracks onto the spectrometer. Tracks 3,4 , and 5 are charged hadrons from the primary vertex. Track 2 is an identified muon with three hits in the muon ID slightly above the projection shown. Track 1 is the candidate charm tracks, which decayed into track 6 , an electron. There is showering in the scintillating fibers and activity in the drift chambers, EMCAL, and muon ID. The electromagnetic energy of this event is $49.2 \pm 5.6 \mathrm{GeV}$, as measured by the EMCAL. 


\section{E872 Run=2846 Event= 9042 Wght= 1.0 U View}
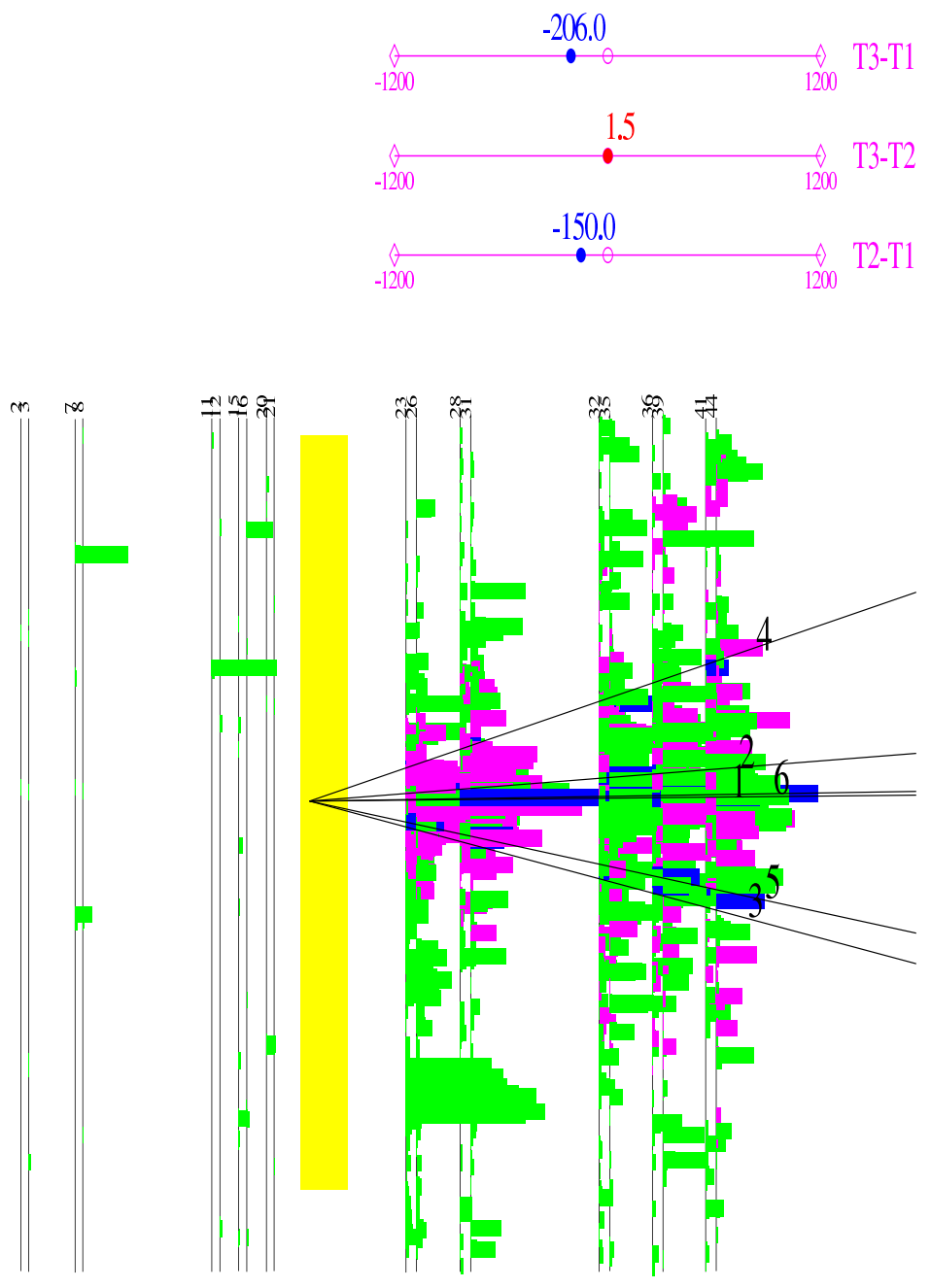

Figure 5.34: The U-view in the spectrometer for charm candidate 2846_09042. Tracks 3,4 , and 5 are charged hadrons from the primary vertex. Track 2 is a muon, identified with the muon ID. Track 1 is the candidate charm tracks, which decayed into track 6 , an electron. Showering exists in the scintillating fibers along track 6, which is the daughter electron. 


\section{E872 Run=2846 Event= 9042 Wght= 1.0 V View}
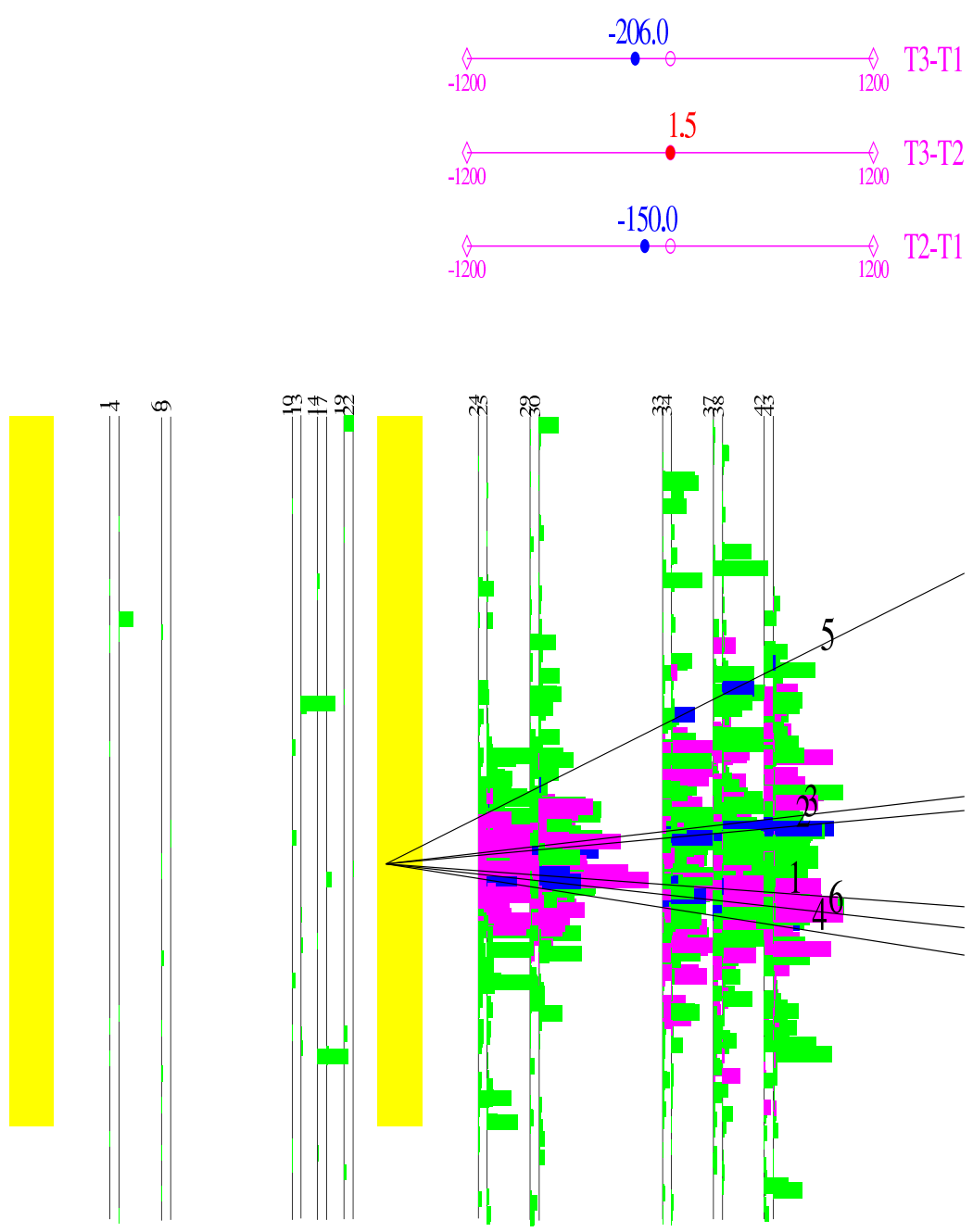

Figure 5.35: The V-view in the spectrometer for charm candidate 2846_09042. 


\section{6_09042}

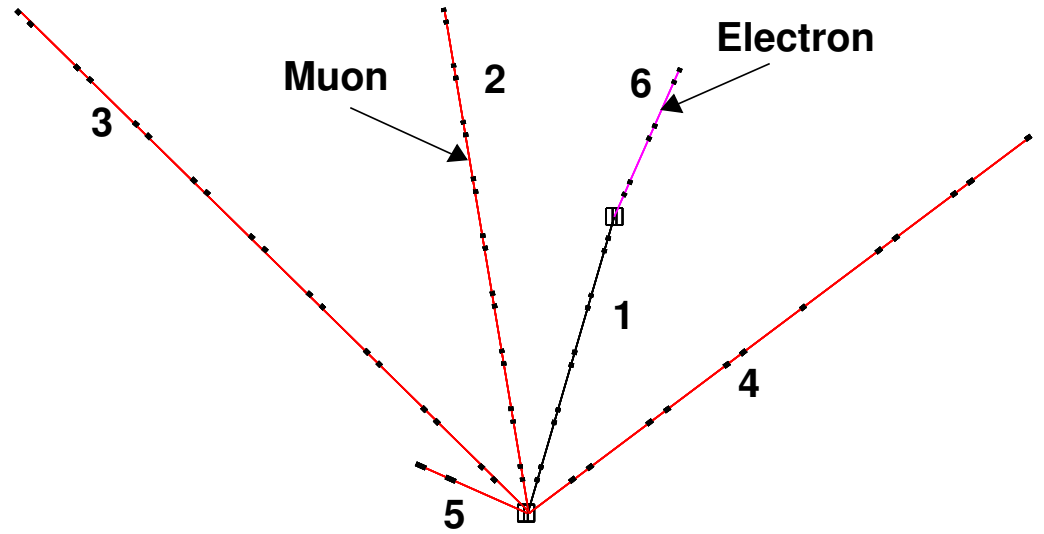

Figure 5.36: The emulsion data for charm candidate 2846_09042. Primary tracks 3, 4, and 5 are hadrons. Track 2 is a primary muon, identified in the muon ID; track 1 is the charm candidate which decayed into an electron, identified in the emulsion. The parameters and momentum measurements for this event are listed in Table 5.15 and 5.16. The neutrino interaction in ECC200 type emulsion, and the decay occurred in steel. 
Parameters of Charm Candidate 3065_03238

\begin{tabular}{|c|c|c|c|c|}
\hline$\theta(\mathrm{rad})$ & $\Delta \phi(\mathrm{rad})$ & $L(\mathrm{~mm})$ & $\alpha(\mathrm{rad})$ & Decay Material \\
\hline 0.250 & 0.71 & 2.10 & 0.229 & steel \\
\hline
\end{tabular}

Table 5.17: Charm kink candidate 3065_03238.

Track Information for Charm Candidate 3065_03238

\begin{tabular}{|c|c|c|c|c|}
\hline 3065_03238 & \multicolumn{2}{|c|}{ Momentum $(\mathrm{GeV})$} & \multicolumn{2}{|c|}{ Identification } \\
\hline Track & MS & Spec & Particle & \\
\hline 1b & $4.4_{-0.9}^{+1.5}$ & & hadron & \\
2b & $7.6_{-1.7}^{+2.8}$ & & hadron & \\
3b & $15.1_{-1.9}^{+4.6}$ & & hadron & \\
4b & $10_{-1.9}^{+5.2}$ & & hadron & \\
5b & & $150 \pm 95$ & hadron & \\
6b & $6.6_{-3.1}^{+5.4}$ & $6 \pm 1$ & hadron & \\
7b & $0.6_{-0.2}^{+0.3}$ & & hadron & \\
$8 \mathrm{~b}$ & & $61 \pm 15$ & electron & \\
$9 \mathrm{~b} / 1 \mathrm{a}$ & & & charm & parent \\
2a & $1.2_{-0.3}^{+0.4}$ & & hadron & daughter \\
\hline
\end{tabular}

Table 5.18: Charm candidate 3065_03238 includes seven charged primary tracks. One of the tracks is a charm candidate, track $9 \mathrm{~b} / 1 \mathrm{a}$, which decayed to or interacted to produce a hadron. One of the other primary tracks is an electron, as identified through pair production. Tracks $5 \mathrm{~b}, 6 \mathrm{~b}$, and $8 \mathrm{~b}$ were fit in the spectrometer, which provided momentum measurements. Multiple scattering measurements were unavailable for $5 \mathrm{~b}$ and $8 \mathrm{~b}$.

\section{Charm Candidate 3065_03238}

In charm candidate 3065_03238, a neutrino interacted in the second emulsion module and produced seven charged primary tracks. Two sets of spectrometer data are included for this event. The first set shows the primary tracks, which are labeled with a "b". The second set shows the parent track and the daughter track, which are labeled with an "a". One of the tracks is a charm candidate, track $9 \mathrm{~b} / 1 \mathrm{a}$ which decayed to or interacted to produce a hadron. One of the other primary tracks is an electron, as identified through pair production. The parameters of this event are summarized in Table 5.17. Multiple scattering measurements were available for all tracks, except $5 \mathrm{~b}$ and $8 \mathrm{~b}$, which both have spectrometer momentum measurement; these measurement are listed in Table 5.18. Fig. 
5.37, Fig. 5.38, and Fig. 5.39 show the $\mathrm{X}$-view, U-view, and V-view of the spectrometer with the projections of the tracks from the primary vertex. Fig. 5.40, Fig. 5.41, and Fig. 5.42 show the $\mathrm{X}$-view, U-view, and $\mathrm{V}$-view of the spectrometer with the the projections of the tracks from the secondary vertex. As Fig. 5.37 and Fig. 5.40 show, there is showering present in the scintillating fibers and activity in the drift chambers and EMCAL. The electromagnetic energy of this event is $121.1 \pm 13.2 \mathrm{GeV}$, as measured by the EMCAL. Fig. 5.43 shows the emulsion data for this event. 


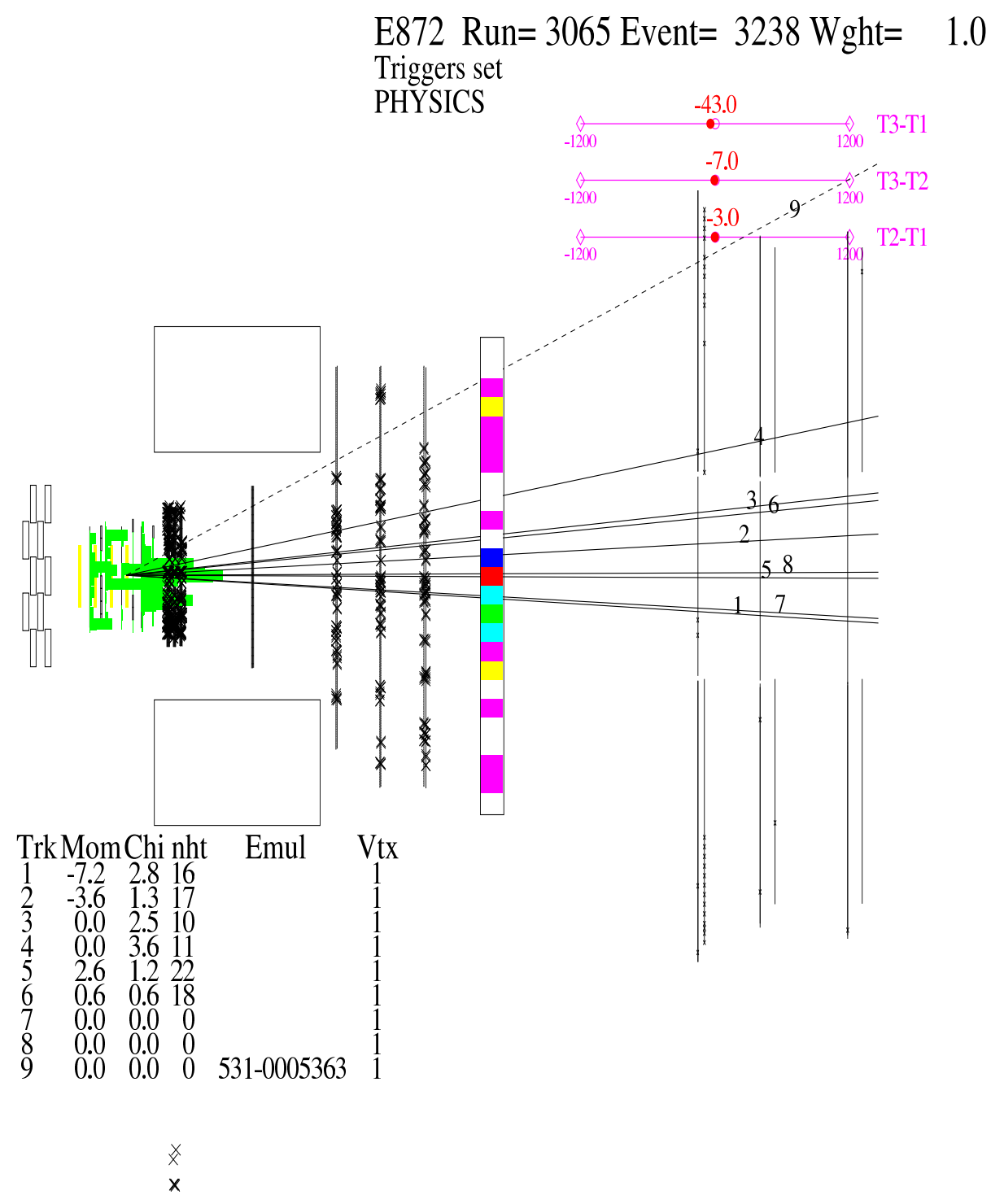

Figure 5.37: The X-view of the spectrometer data for charm candidate 3065_03238 with the projections of the tracks from the primary vertex. The tracks shown are emulsion tracks projected into the spectrometer. All tracks except track 8 and track 9 are hadrons. Track 8 is an electron, which was identified through pair production in the emulsion. Track 9 is the candidate charm tracks, which decayed to or interacted to produce track 2a, as shown in Fig. 5.40. There is showering present in the scintillating fibers and activity in the drift chambers and EMCAL. The electromagnetic energy of this event is $121.1 \pm 13.2$ $\mathrm{GeV}$, as measured by the EMCAL. 


\section{E872 Run= 3065 Event $=3238 \mathrm{Wght}=1.0$}
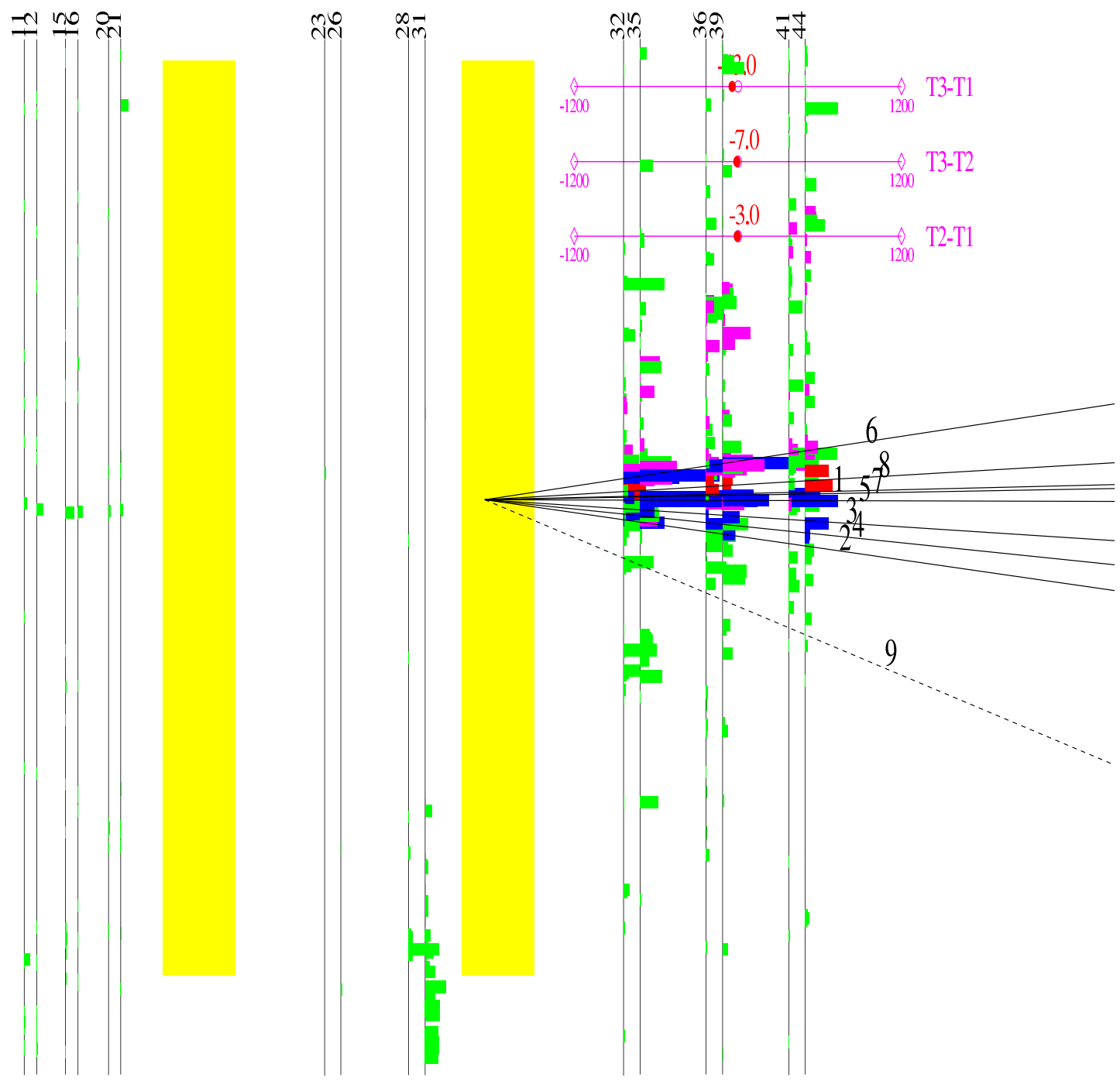

Figure 5.38: The U-view of the spectrometer data for charm candidate 3065_03238 with the projections of the tracks from the primary vertex. All tracks except track 8 and track 9 are hadrons. Track 8 is an electron identified through pair production in the emulsion. Track 9 is the candidate charm tracks, which decayed to or interacted to produce track 2a, as shown in Fig. 5.40. 


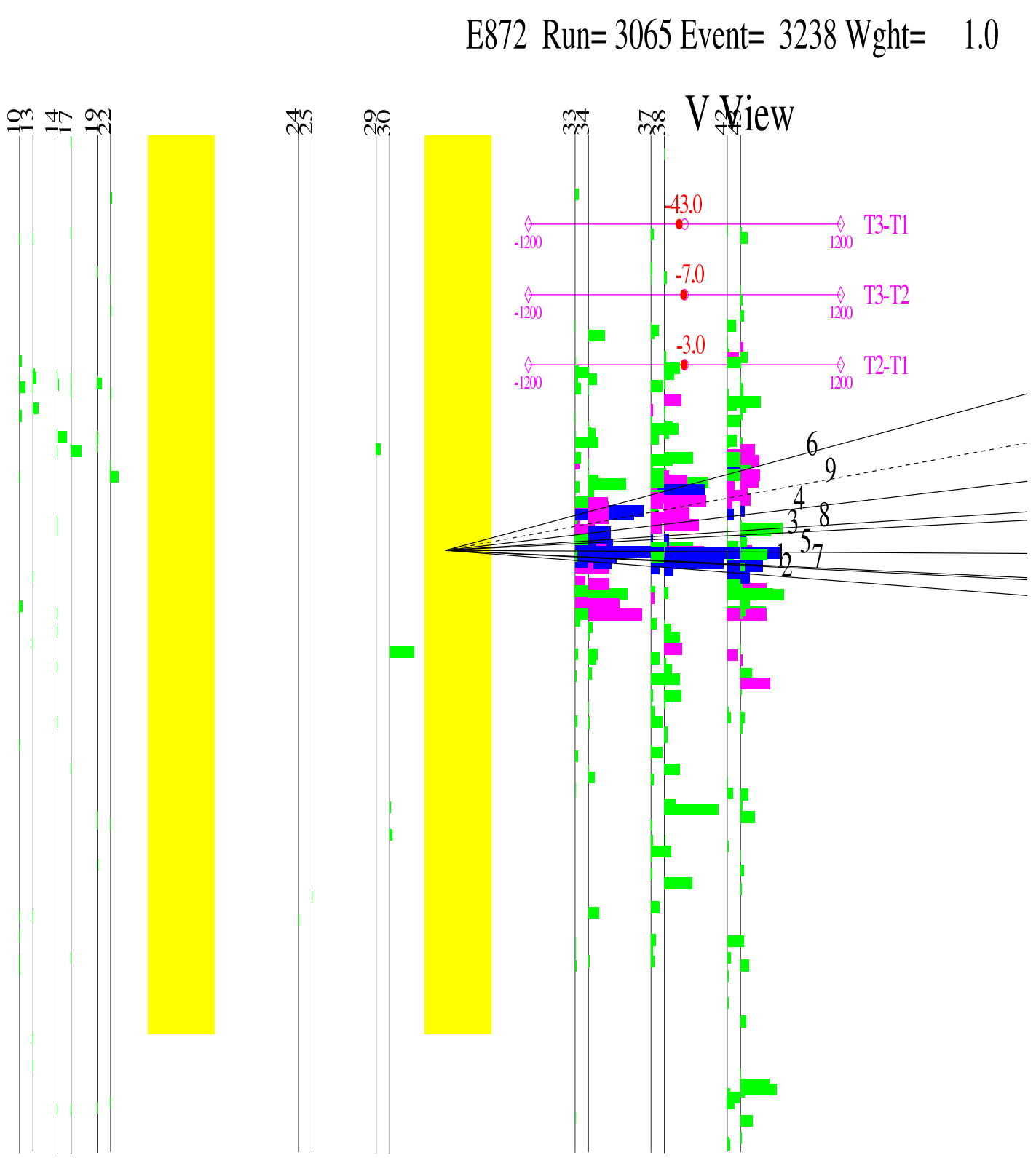

Figure 5.39: The V-view of the spectrometer data for charm candidate 3065_03238 with the projections of the tracks from the primary vertex. 


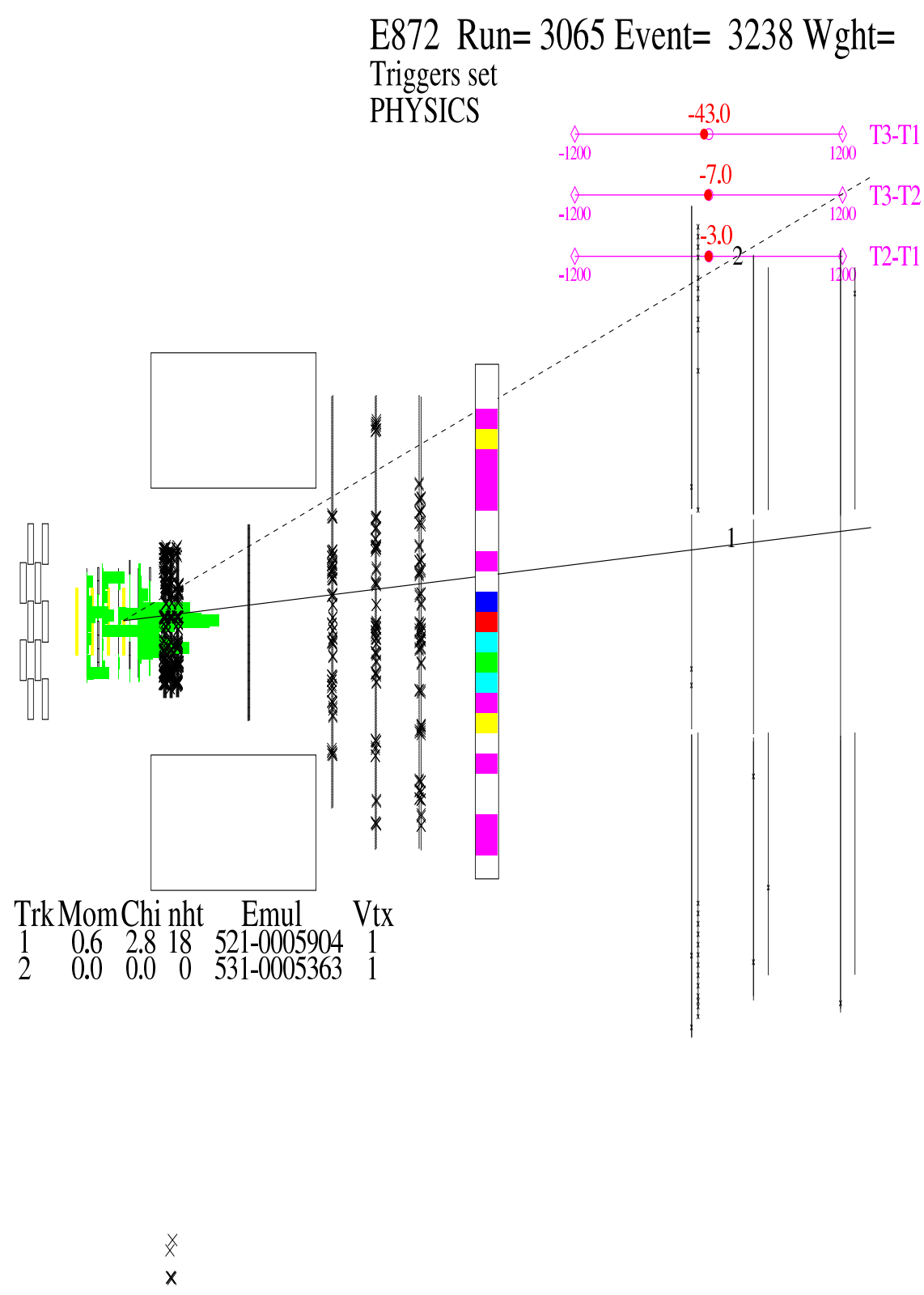

Figure 5.40: The X-view of the spectrometer data for charm candidate 3065_03238 with the projections of the tracks from the secondary vertex. The tracks shown are emulsion tracks projected into the spectrometer. Track 2 is the candidate charm track, and track 1 is the daughter. 


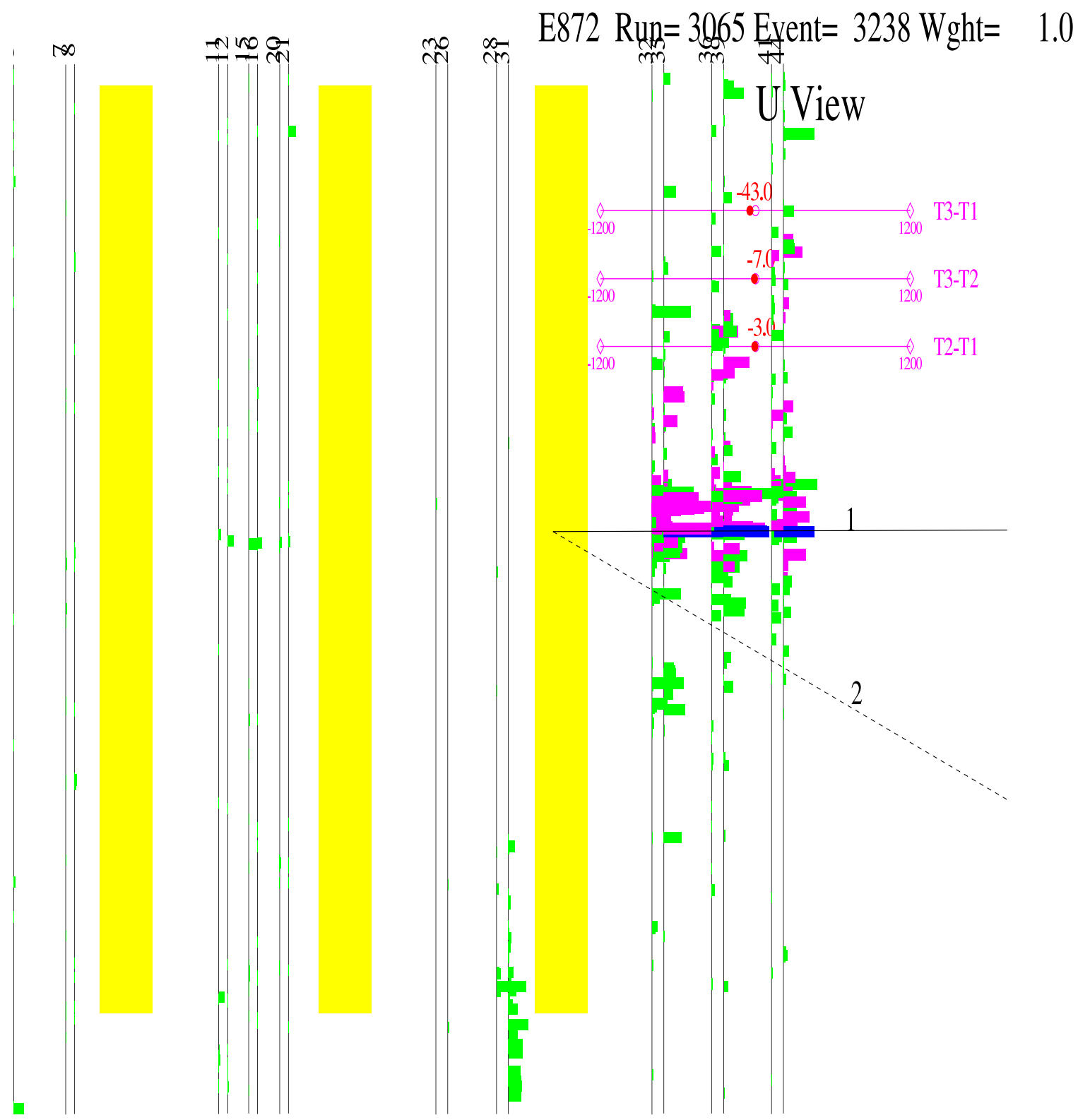

Figure 5.41: The U-view of the spectrometer data for charm candidate 3065_03238 with the projections of the tracks from the secondary vertex. Track 2 is the candidate charm track, and track 1 is the daughter. 
E872 Run= 3065 Event $=3238$ Wght= 1.0
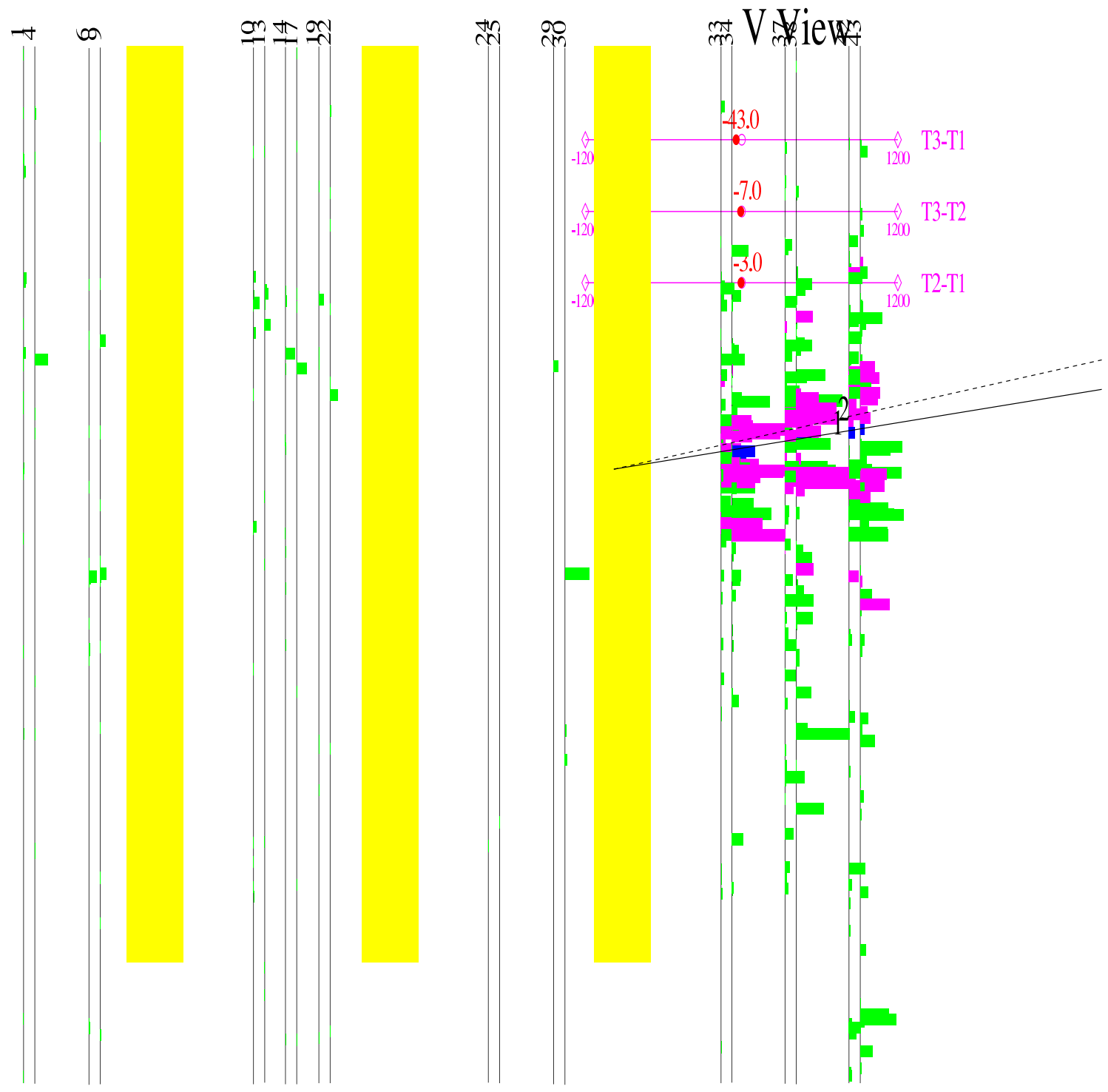

Figure 5.42: The V-view of the spectrometer data for charm candidate 3065_03238 with the projections of the tracks from the secondary vertex. 


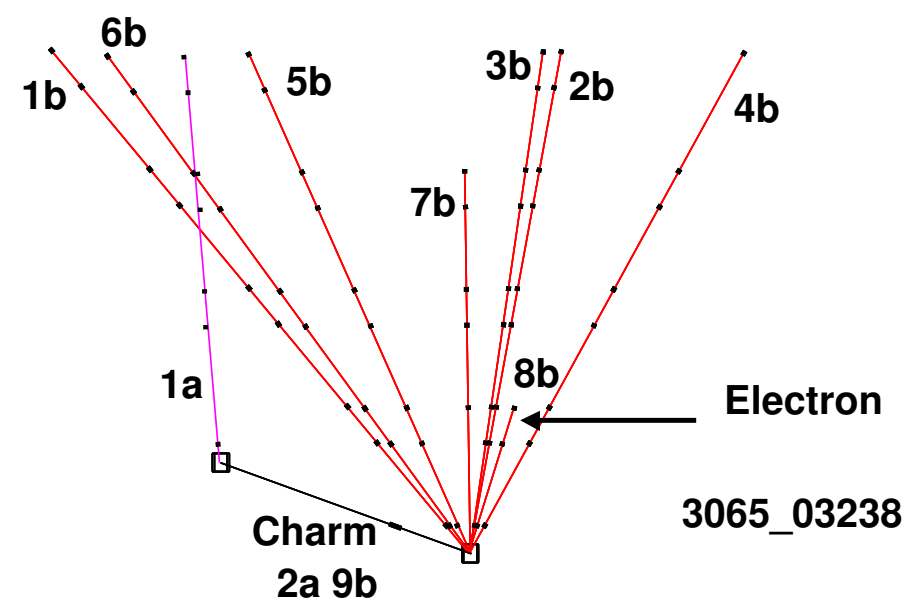

Figure 5.43: Emulsion data for charm candidate 3065_03238. In the emulsion picture, each primary track, shown in the first set of spectrometer figures, are designated by an "b". The parent and daughter track, shown in the second set of spectrometer figures, are designated with an "a". All tracks are hadrons, except for $2 \mathrm{a} / 9 \mathrm{~b}$, which is the charm candidate, and $8 \mathrm{~b}$, which is an electron identified in the emulsion. The parameters and momentum measurements for this event are listed in Table 5.17 and 5.18. The neutrino interacted in ECC200 type emulsion, and the decay occurred in steel. 


\section{Parameters of Charm Candidate 3227_03420}

\begin{tabular}{|c|c|c|c|c|}
\hline$\theta(\mathrm{rad})$ & $\Delta \phi(\mathrm{rad})$ & $L(\mathrm{~mm})$ & $\alpha(\mathrm{rad})$ & Decay Material \\
\hline 0.010 & 1.90 & 1.8 & 0.070 & steel \\
\hline
\end{tabular}

Table 5.19: Charm kink candidate 3227_03420.

Track Information for Charm Candidate 3227_03420

\begin{tabular}{|c|c|c|c|c|}
\hline 3227_03420 & \multicolumn{2}{|c|}{ Momentum $(\mathrm{GeV})$} & \multicolumn{2}{|c|}{ Identification } \\
\hline Track & MS & Spec & Particle & \\
\hline 1 & & $23 \pm 3$ & electron & \\
2 & & & charm & parent \\
3 & $4.3_{-1.4}^{+3.4}$ & & hadron & daughter \\
\hline
\end{tabular}

Table 5.20: Charm candidate 3227_03420 includes two charged primary tracks. One of the tracks is a charm candidate, track 2 , which decayed to or interacted to produce a hadron, track 3. One of the other primary tracks is a electron, as identified through pair production in the emulsion. Track 1 currently has no multiple scattering momentum measurement, but has a spectrometer momentum measurement.

\section{Charm Candidate 3227_03420}

In charm candidate 3227_03420, a neutrino interacted in the first emulsion module and produced two charged primary tracks. One of the tracks is a charm candidate, track 2, which decayed to or interacted to produce a hadron, track 3. One of the other primary tracks is a electron, as identified through pair production in the emulsion. The parameters of this event are summarized in Table 5.19. The momentum of track 1 was measured using the spectrometer, and the momentum of track 3 was measured using multiple scattering; the measurements are listed in Table 5.20. Fig. 5.44, Fig. 5.45, and Fig. 5.46 show the $\mathrm{X}$-view, U-view, and V-view of the spectrometer. As Fig. 5.44 shows, there is showering in the scintillating fibers and a small amount of activity in the drift chambers and the EMCAL. The total electromagnetic energy of this event is $4.3 \pm 0.7 \mathrm{GeV}$, as measured in the EMCAL. Fig. 5.45 and Fig. 5.46 show the scintillating fiber data for this event. There is showering along track 1, which is the electron. Fig. 5.47 shows the emulsion data for this event. 


\section{E872 Run= 3227 Event= $3420 \mathrm{Wght}=1.0$ Triggers set} PHYSICS

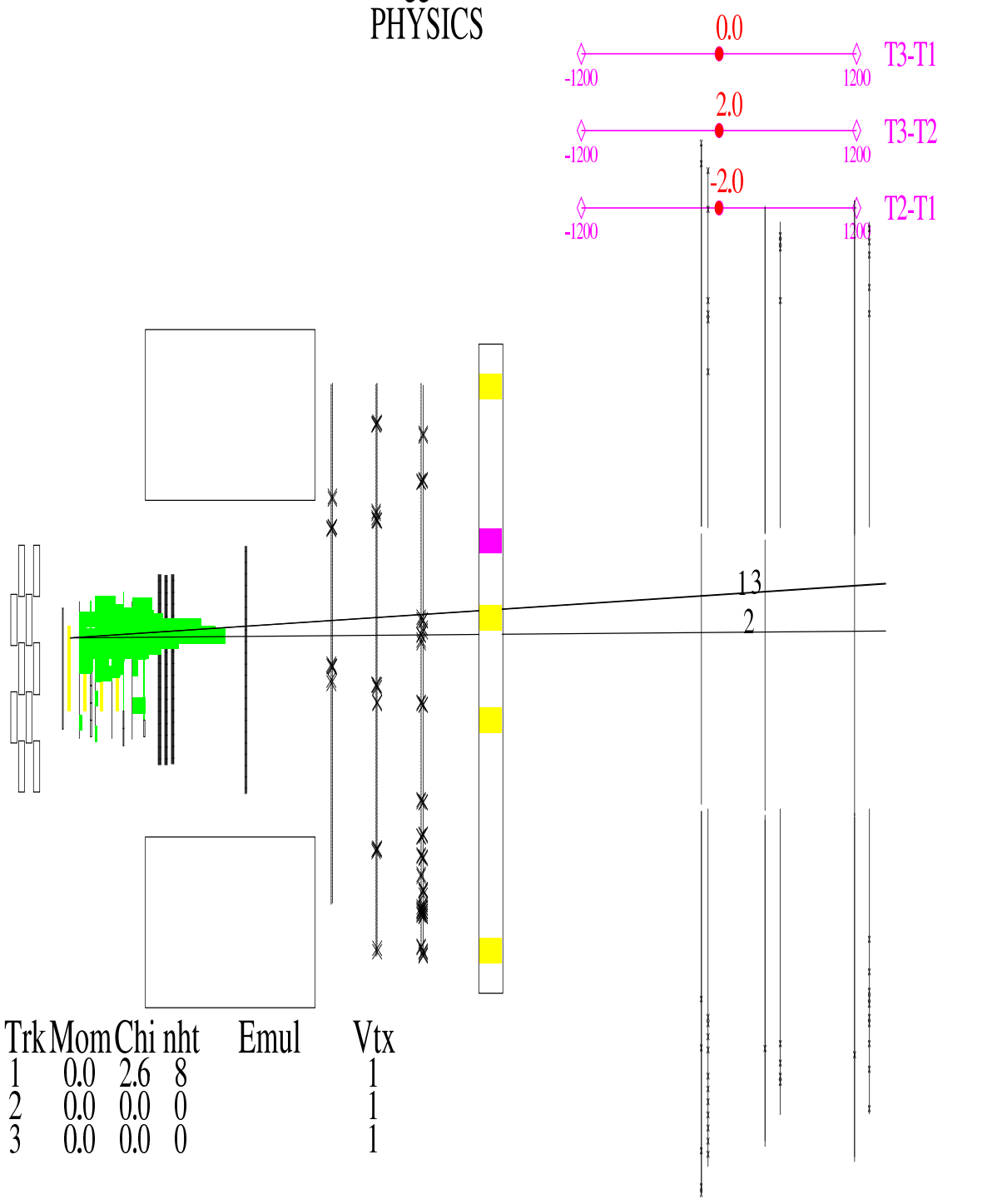

Figure 5.44: The X-view of the spectrometer data for charm candidate 3227_03420. The tracks shown are the projections of the emulsion tracks onto the spectrometer. Track 1 is an electron. Track 2 is the charm candidate that decayed to or interacted to produce track 3. There is showering in the scintillating fibers and a small amount of activity in the drift chambers and the EMCAL. The total electromagnetic energy of this event is $4.3 \pm 0.7$ $\mathrm{GeV}$, as measured in the EMCAL. Tracks 1 and 3 overlap in this view, but not in the other views. 


\section{E872 Run= 3227 Event= 3420 Wght= 1.0 U View}
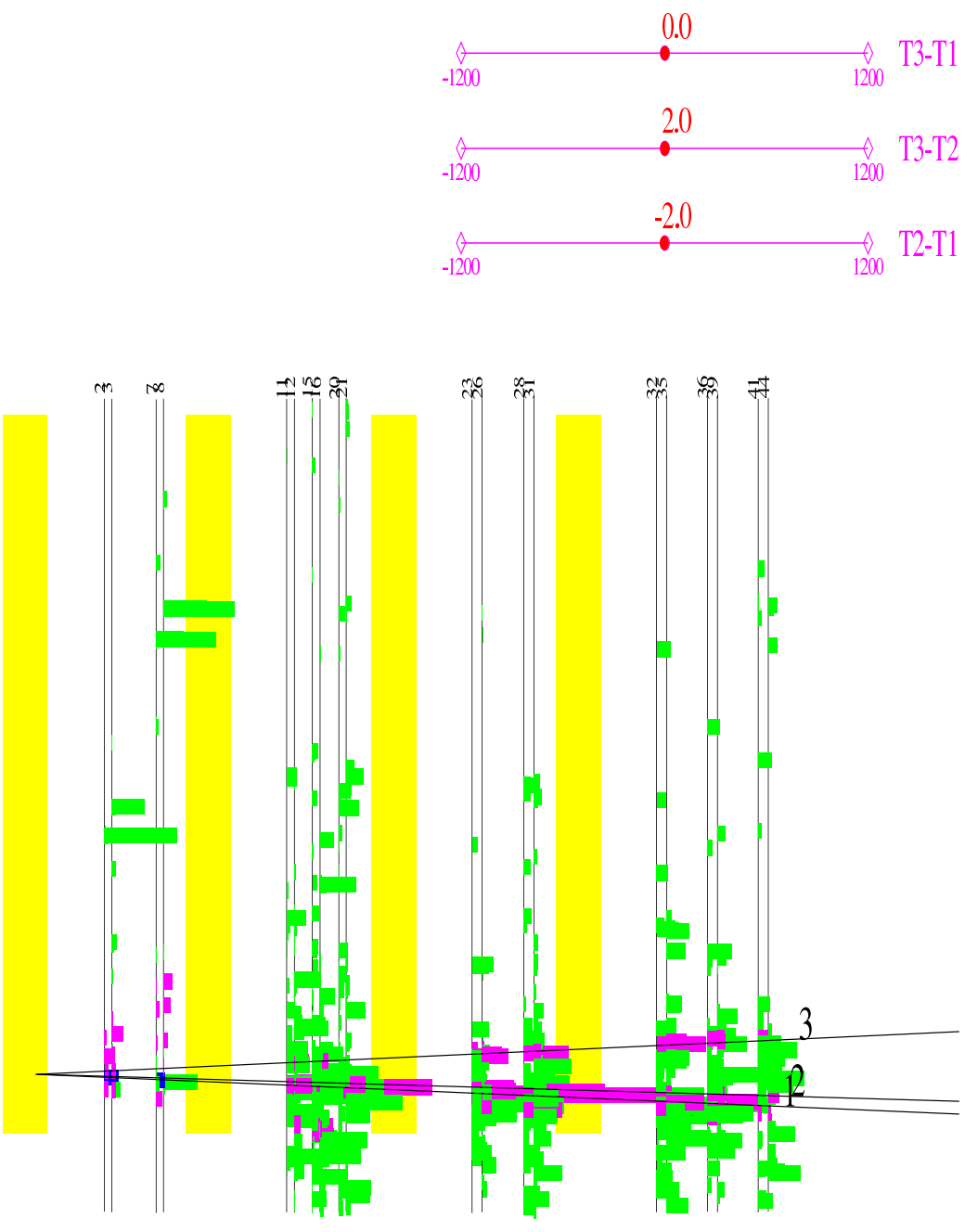

Figure 5.45: The U-view of the spectrometer data for charm candidate 3227_03420. Track 2 is the charm candidate that decayed to or interacted to produce track 3 . There is showering along track 1 , which is the primary electron. 


\section{E872 Run= 3227 Event= 3420 Wght= 1.0 V View}
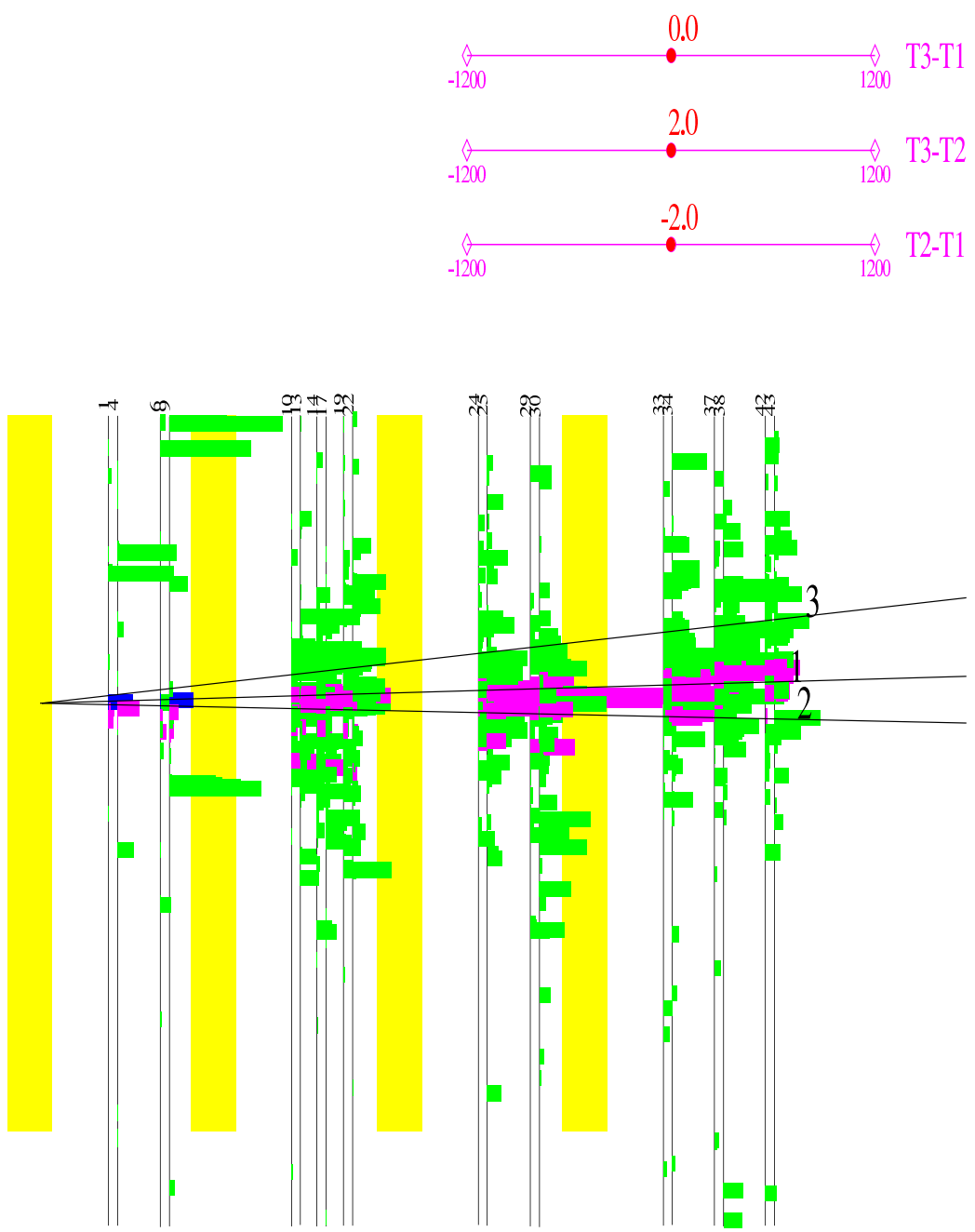

Figure 5.46: The V-view of spectrometer data charm candidate 3227_03420. 


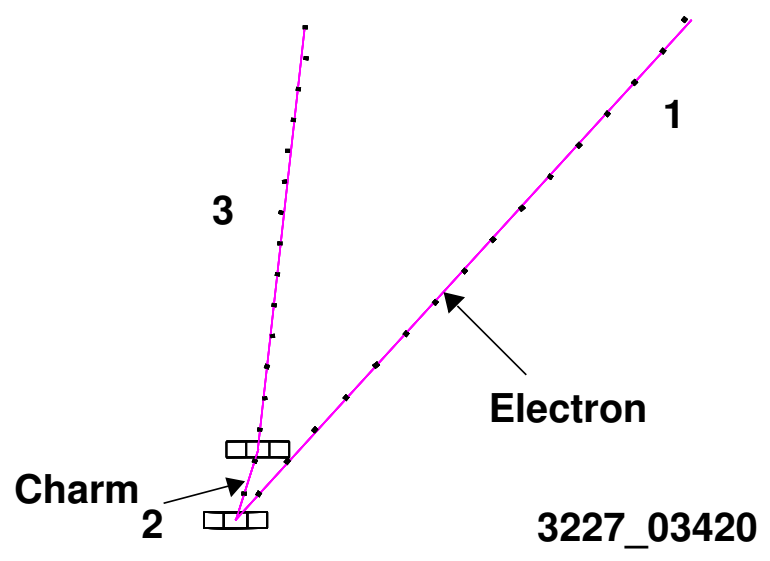

Figure 5.47: Emulsion data for charm candidate 3227_03420. Track 1 is an electron. Track 2 is the candidate charm track, which decayed to or interacted to produce track 3 . The parameters and momentum measurements for this event are listed in Table 5.19 and 5.20. This event occurred in ECC800 type emulsion, and the decay occurred in steel. 
Parameters of Charm Candidate 3245_22786

\begin{tabular}{|c|c|c|c|c|}
\hline$\theta(\mathrm{rad})$ & $\Delta \phi(\mathrm{rad})$ & $L(\mathrm{~mm})$ & $\Sigma I P$ & Decay Material \\
\hline 0.142 & 1.60 & 0.40 & 0.013 & emulsion \\
\hline
\end{tabular}

Table 5.21: Charm trident candidate 3245_22786.

Track Information for Charm Candidate 3245_22786

\begin{tabular}{|c|c|c|c|c|}
\hline 3245_22786 & \multicolumn{2}{|c|}{ Momentum $(\mathrm{GeV})$} & \multicolumn{2}{|c|}{ Identification } \\
\hline Track & MS & Spec & Particle & \\
\hline 1 & $9.7_{-5}^{+49}$ & $30 \pm 33$ & muon & \\
2 & $2.3_{-0.7}^{+1.6}$ & & hadron & \\
3 & $1.3_{-0.3}^{+0.6}$ & & hadron & \\
4 & $4.9_{-1.4}^{+2.8}$ & & & daughter \\
5 & & $26 \pm 13$ & & daughter \\
6 & & & charm & parent \\
\hline
\end{tabular}

Table 5.22: Charm candidate 3245_22786 includes four charged primary tracks. One of the tracks is a charm candidate, track 6 , which decayed to or interacted to produce three charged particles, track 4, track 5, and a track with an angle larger than 400 mrad. Since $400 \mathrm{mrad}$ is the threshold angle of the automated scanner, this daughter track is not visible in the emulsion. One of the primary tracks is a muon, track 1, as identified using the muon ID. Tracks 1 and 5 were fit in the spectrometer, which provided spectrometer momentum measurements.

\section{Charm Candidate 3245_22786}

In charm candidate 3245_22786, a neutrino interacted in the second emulsion module and produced four charged tracks. One of the tracks is a charm candidate, track 6 , which decayed to or interacted to produced three charged particles, track 4,5 , and a track with an angle larger than $400 \mathrm{mrad}$. Since $400 \mathrm{mrad}$ is the threshold angle of the automated scanner, this daughter track is not visible in the emulsion. One of the primary tracks is a muon, track 1, as identified using the muon ID. The parameters of this event are summarized in Table 5.21. Multiple scattering momentum measurements were available for all but track 5 . The momenta of tracks 1 and 5 were measured in the spectrometer. The momentum measurements are listed in Table 5.22. Fig. 5.48, Fig. 5.49, and Fig. 5.50 show the X-view, U-view, and V-view of the spectrometer. As Fig. 5.48 shows, there is activity in the scintillating fibers, drift chamber, and the EMCAL. The total 


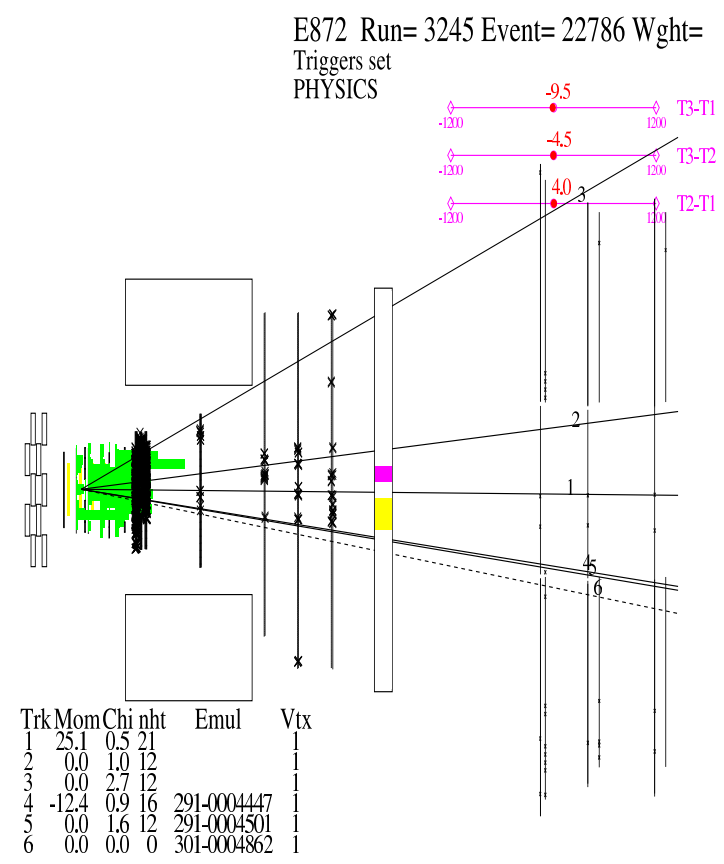

Figure 5.48: The $\mathrm{X}$-view of the spectrometer data for charm candidate $3245 \_22786$. The tracks shown are the projections of the emulsion tracks onto the spectrometer. Tracks 2 and 3 are charged hadrons. Track 6 is the charm candidate which decayed to or interacted to produce tracks 2,3 , and a track not visible in the emulsion data because of its large angle. There is activity in the scintillating fibers, drift chamber, and the EMCAL. The total electromagnetic energy of this event is $2.5 \pm 0.4 \mathrm{GeV}$, as measured with the EMCAL. Track 1 is a muon, with hits in the muon ID. Tracks 4 and 5 overlap in this view, but not in the other views.

electromagnetic energy of this event is $2.5 \pm 0.4 \mathrm{GeV}$, as measured with the EMCAL. Fig. 5.49 and Fig. 5.50 show the scintillating fiber data. Fig. 5.51 shows the emulsion data for this event. The missing track was identified in the emulsion manually. 


\section{E872 Run= 3245 Event $=22786$ Wght= 1.0 U View}
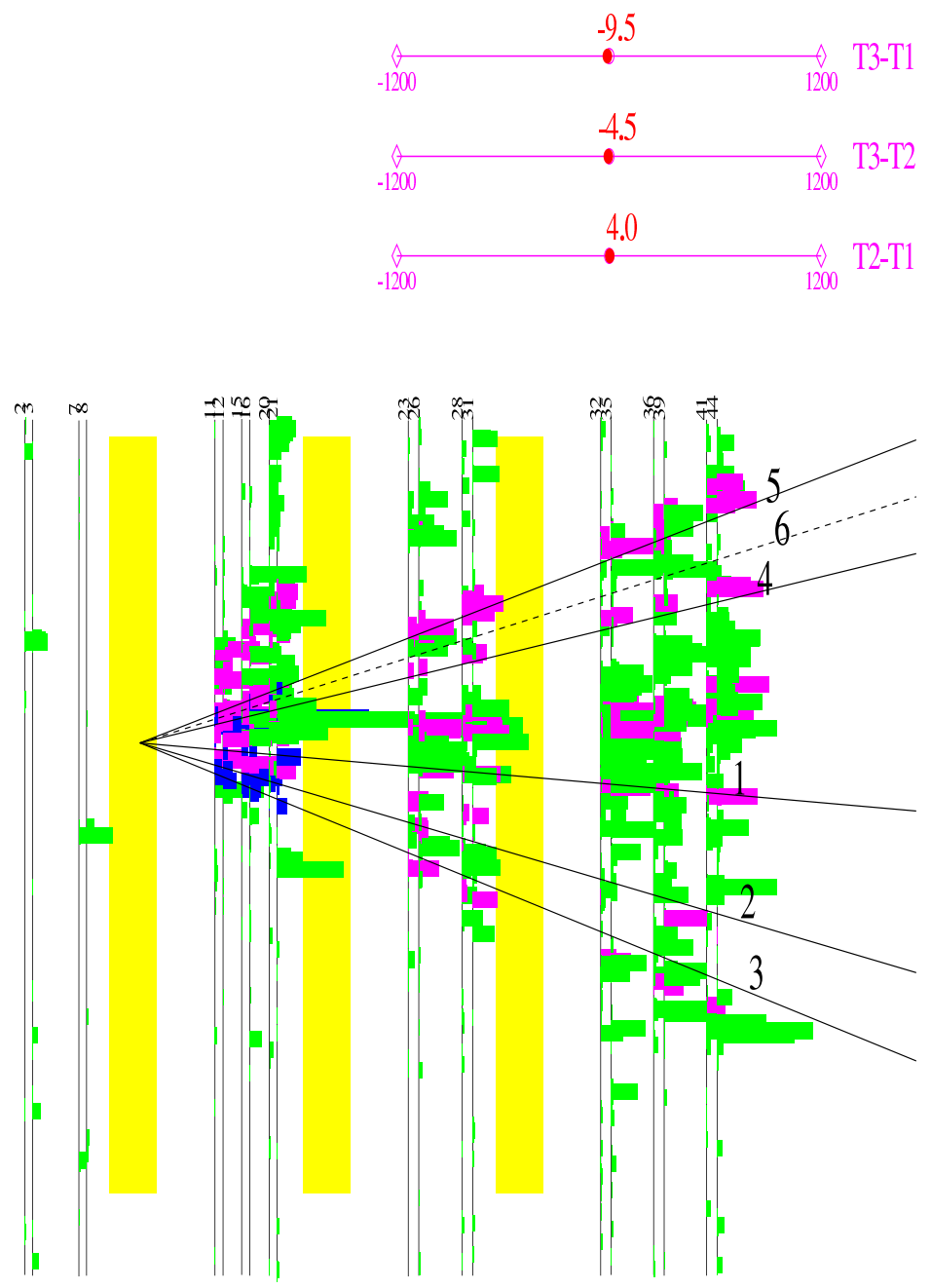

Figure 5.49: The U-view of the spectrometer data for charm candidate 3245_22786. Tracks 2 and 3 are charged hadrons. Track 6 is the charm candidate which decayed to or interacted to produce tracks 2,3 , and a track not visible in the emulsion data because of its large angle. 


\section{E872 Run= 3245 Event= 22786 Wght= 1.0 V View}
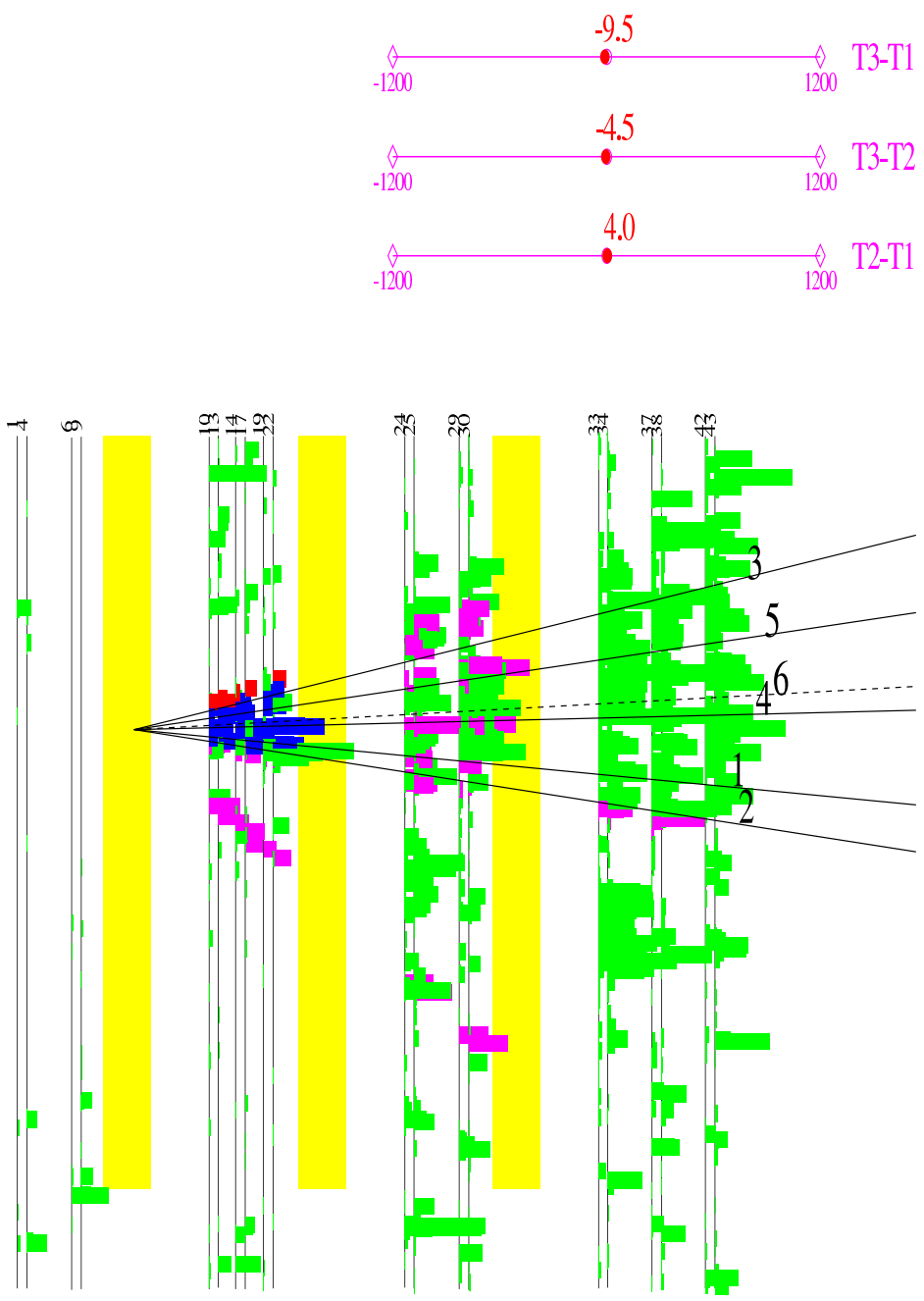

Figure 5.50: The V-view of the spectrometer data for charm candidate 3245_22786. 


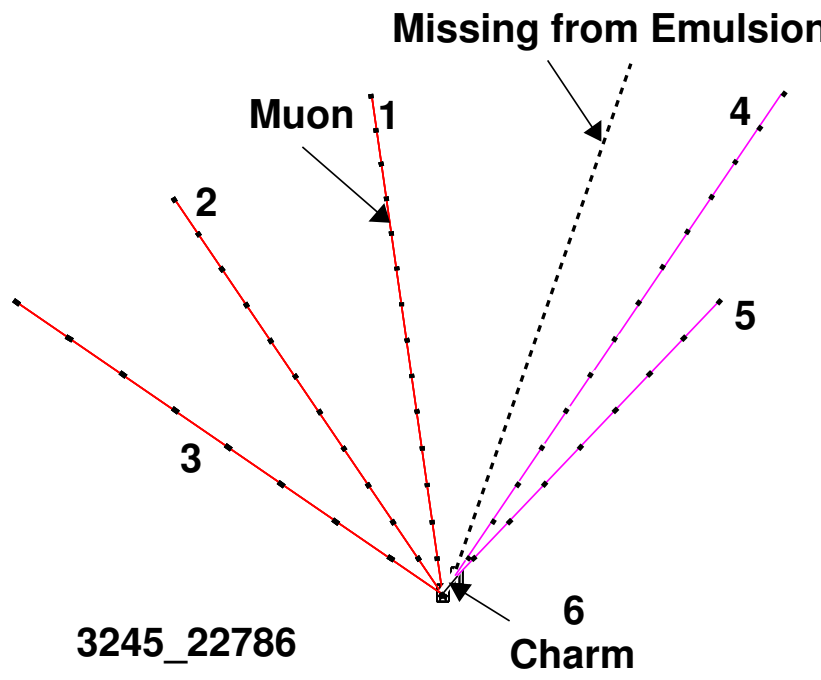

Figure 5.51: Emulsion data for charm candidate 3245_22786. Tracks 2 and 3 are primary hadrons; track 6 is the charm candidate which decayed to or interacted to produce tracks 2,3 , and a track not visible in the emulsion because of its large angle. This track is added manually in the figure above. The parameters and momentum measurements for this event are listed in Table 5.21 and 5.22. The neutrino interacted in the ECC800 type of emulsion, and the decay occurred in emulsion. 


\subsection{Statistical Analysis of Tau and Charm Candidates}

The statistical analysis performed on the tau candidates provides the relative probability that each candidate is caused by a tau neutrino interaction or a background source. These relative probabilities are calculated using Bayes's theorem,

$$
P(\{x\} \mid i)=\frac{\Gamma_{i} \Pi(\{x\} \mid i)}{\Gamma_{i} \Pi(\{x\} \mid i)+\Sigma_{\mathrm{bkg}} \Gamma_{\mathrm{bkg}} \Pi(\{x\} \mid \mathrm{bkg})},
$$

where $\Gamma_{i}$ is the prior probability of an event type $i$ and $\Pi(\{x\} \mid i)$ is the probability density function evaluated at a set $\{x\}$, where $\{x\}$ is a set of five parameters for the kink events and four parameters for trident events.

$$
\begin{aligned}
& \{x\}=\left(\theta, \Delta \phi, L, \alpha, P_{\mathrm{d}}\right) \text { for kink events, and } \\
& \{x\}=(\theta, \Delta \phi, L, \Sigma I P) \text { for trident events, }
\end{aligned}
$$

where $\theta$ is the production angle, $\Delta \phi$ is the polar angle asymmetry, $L$ is the decay length, $\alpha$ is the kink angle, $P_{\mathrm{d}}$ is the momentum of the daughter, and $\Sigma I P$ is the sum of the daughter impact parameters. These parameters were discussed in Sec. 4.5.

The prior probability is the apriori knowledge of the likelihood of a particular hypothesis. This analysis considered three hypothesis: a tau neutrino interaction with a tau decay, a neutrino interaction with a charged charm decay, and a neutrino interaction with a hadronic interaction. Only the charm and interaction backgrounds were considered because they accounted for $97 \%$ of all backgrounds to the tau signal [29]. The apriori knowlegde of an event depends upon the daughter type and the material in which the secondary vertex occurred. The probabilty density was calculated using simulated data for each hypothesis. The probability density evaluated for a paticular set of values, is determined from the fraction of simulated events which lay within a small region of parameter space centered on those values. This analysis was performed on all events which satisfied either the tau criteria disscussed in Sec. 4.6 .3 or the charged charm criteria discussed in Sec. 4.6.4.

\subsubsection{Prior Probability}

The analysis is performed on four types of candidates: the tau kink candidates, the tau trident candidates, the charm kink candidates, and the charm trident candidate. Since each of these events have different characteristics, each hypothesis, tau, charm, and interaction, will have a separate prior probability for each type of candidate, tau kink, tau trident, charm kink, and charm trident. 


\section{Tau Prior Probabilities}

The tau prior probability for the tau kink candidates, $\Gamma_{\nu_{\tau}}^{\text {kink }}$, is the number of expected tau neutrino interactions divided by the total number of events. If the tau decays to a single charged particle, the prior probability is

$$
\Gamma_{\nu_{\tau}}^{\mathrm{kink}}=\frac{N_{\nu_{\tau}}^{\mathrm{exp}} \operatorname{BR}(\tau \rightarrow \mathrm{kink})}{N_{\text {total }}}
$$

where $N_{\nu_{\tau}}^{\exp }$ is the expected number of tau neutrino charged-current interactions calculated using the theoretical cross section and the data set, $B R(\tau \rightarrow$ kink $)$ is the branching ratio for a tau to decay to a single charged particle, which is $0.8535 \pm 0.0007$ [25], and $N_{\text {total }}$ is the total number of events in the data set, which is 539 events. The expected number of tau neutrino interactions is calculated using the cross section given in Ch. 2, and

$$
N_{\nu_{\tau}}^{\exp }=N_{\text {scat }} N_{\text {pot }} \epsilon_{\nu_{\tau}} \int N_{\nu_{\tau}}^{\operatorname{tar}}(E) \sigma_{\nu_{\tau} N}(E) d E
$$

where these parameters and their values are discussed in Ch. 6. Using this cross section could introduce uncertainty in the measurement, but, for this to be a factor, the cross section would have to be several orders of magnitude different from the prediction. This analysis shows that to be unlikely. The resulting number of expected tau neutrino interactions is $10 \pm 2$ events. The prior probability for the tau kink candidates is

$$
\Gamma_{\nu_{\tau}}^{\mathrm{kink}}=\frac{10 \times 0.8535}{539}=1.6 \times 10^{-2} .
$$

The tau prior probability for the trident tau candidates, $\Gamma_{\tau}^{\text {tri }}$, is calculated in a similar manner, replacing the branching ratio for single prong tau decays with the branching ratio for trident tau decay,

$$
\Gamma_{\nu_{\tau}}^{\mathrm{tri}}=\frac{N_{\nu_{\tau}}^{\exp } B R(\tau \rightarrow \text { trident })}{N_{\text {total }}}=2.7 \times 10^{-3}
$$

Since the charm kink and charm trident candidates have an identified primary lepton, the tau prior probabilities for these candidates are zero. All tau prior probabilities are listed in Table 5.29.

\section{Charm Prior Probabilities}

The charm prior probability for the charm kink candidates, $\Gamma_{\mathrm{c}}^{\mathrm{kink}}$, is

$$
\Gamma_{\mathrm{c}}^{\mathrm{kink}}=\frac{\sum_{i} N_{\mathrm{c}_{\mathrm{i}}}^{\exp } B R\left(\mathrm{c}_{\mathrm{i}} \rightarrow \text { kink }\right) \chi}{N_{\text {total }}},
$$




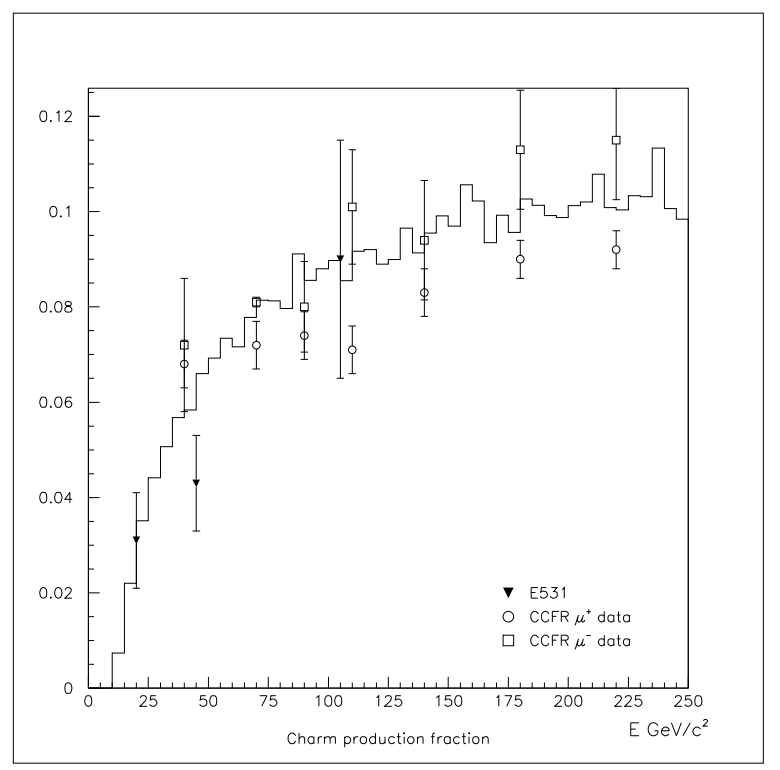

Figure 5.52: Charm production in neutrino-nucleon interactions.

where $N_{\mathrm{c}_{\mathrm{i}}}^{\exp }$ is the expected number of charm particles of type $i, B R\left(\mathrm{c}_{\mathrm{i}} \rightarrow\right.$ kink $)$ is the branching ratio for a charm particle of type $i$ to decay a single charged particle, and $\chi$ is the probability that the charm event passed all of the tau criteria, calculated using the Monte Carlo. The selection criteria are summarized in Sec. 4.6.3. The expected number of charm particles of type $i$ is

$$
N_{\mathrm{c}_{\mathrm{i}}}^{\exp }=N_{c c} F \beta_{i},
$$

where $N_{c c}$ is the number of charged-current muon and electron neutrino interactions in the data set, $F$ is the fraction of neutrino interactions which produce a charm quark, and $\beta_{i}$ is the probability of the charm quark resulting in a hadron of type $i$, where $i$ is $D^{ \pm}, D_{s}$, or $\Lambda_{c}$. Excited states, such as $D^{* \pm}$ and $D^{* 0}$, are also included, as discussed in Sec. 3.2.1. 376 events out of 539 events were identified as charged-current interactions. The ratio of charm production to the total charged-current neutrino interaction rate was calculated using the LEPTO [37] event generator. The ratio of charm particle production to the total neutrinonucleon charged-current interaction rate is shown in Fig. 5.52. The LEPTO output was scaled to match the results of the two experiments that have measured neutrino charm production [43] [44]. When this distribution was integrated over the energy spectrum for the specific neutrino beam in this experiment, the average charm particle production 


\section{Charm Probabilities}

\begin{tabular}{|c|c|}
\hline Particle & $\beta_{i}$ \\
\hline$D^{ \pm}$ & $0.24 \pm 0.06$ \\
\hline$D_{s}^{ \pm}$ & $0.09 \pm 0.03$ \\
\hline$\Lambda_{c}^{+}$ & $0.10 \pm 0.03$ \\
\hline
\end{tabular}

Table 5.23: The probability of a charm quark producing a $D^{ \pm}, D^{0}$, and $\Lambda_{c}$ [45].

Charm Prior Probabilities for Tau Kink Candidates

\begin{tabular}{|c|c|c|c|c|}
\hline Particle & $N_{\mathrm{c}}^{\exp }$ & $B R\left(\mathrm{c}_{\mathrm{i}} \rightarrow \mathrm{kink}\right)$ & $\chi$ & $\Gamma_{\mathrm{c}}^{\mathrm{kink}}$ \\
\hline$D^{ \pm}$ & $6.0 \pm 1.7$ & $0.46 \pm 0.03$ & 0.132 & $6.8 \pm 1.8 \times 10^{-4}$ \\
\hline$D_{s}^{ \pm}$ & $2.2 \pm 0.3$ & $0.37 \pm 0.03$ & 0.138 & $2.1 \pm 1.5 \times 10^{-4}$ \\
\hline$\Lambda_{c}^{+}$ & $2.5 \pm 0.8$ & $0.65 \pm 0.40$ & 0.096 & $2.9 \pm 1.5 \times 10^{-4}$ \\
\hline Total & & & & $1.2 \pm 0.3 \times 10^{-3}$ \\
\hline
\end{tabular}

Table 5.24: The expected numbers, the single-prong branching ratios [25], the probability of passing the selection criteria [29], and the resulting prior probabilities for each charm species.

fraction $F$ was $0.066 \pm 0.008$ [29]. This total included both charged and neutral charm. Since only the charged charm particles were backgrounds, the distribution of charm species which were produced was also necessary. These production fractions were measured in E531 [43] and recalculated using improved lifetime measurements [25]. $\beta_{i}$, the production fraction, is listed for each charm particle in Table 5.23. $N_{\mathrm{C}_{\mathrm{i}}}^{\exp }$, the single-prong branching ratios for $D^{ \pm}, D^{0}$, and $\Lambda_{c}{ }^{1}, \chi$, and the resulting charm prior probabilities for the tau kink candidates are listed in Table 5.24.

The charm prior probability for tau trident candidates, $\Gamma_{\mathrm{c}}^{\mathrm{tri}}$, was calculated in the same manner described above. $N_{\mathrm{C}_{\mathrm{i}}}^{\text {exp }}$, the trident branching ratios for $D^{ \pm}, D^{0}$, and $\Lambda_{c}, \chi$, and and the resulting charm prior probabilities for the tau trident candidates are listed in Table 5.25.

Since the charm kink and charm trident candidates have an identified primary lepton, the probability of selection, $\chi$, changes in the charm prior probabilities. $\chi$ still requires

\footnotetext{
${ }^{1}$ The branching fractions of $\Lambda_{c}$ have not been studied as thoroughly as the $D$ mesons. All of the multiprong modes were totaled and subtracted from one to give an upper limit on the single-prong branching fraction. The sum of multi-prong modes [25] is $35.4 \pm 3.7 \%$; the maximum single prong branching ratio is $64.6 \pm 3.7 \%$.
} 


\section{Charm Prior Probability for Tau Trident Candidates}

\begin{tabular}{|c|c|c|c|c|}
\hline Particle & $N_{\mathrm{c}}^{\exp }$ & $B R\left(\mathrm{c}_{\mathrm{i}} \rightarrow\right.$ trident $)$ & $\chi$ & $\Gamma_{\mathrm{c}}^{\mathrm{tri}}$ \\
\hline$D^{ \pm}$ & $6.0 \pm 1.7$ & $0.37 \pm 0.19$ & 0.30 & $1.2 \pm 0.7 \times 10^{-3}$ \\
\hline$D_{s}$ & $2.2 \pm 0.3$ & $0.32 \pm 0.23$ & 0.30 & $4.0 \pm 0.4 \times 10^{-4}$ \\
\hline$\Lambda_{c}$ & $2.5 \pm 0.8$ & $0.20 \pm 0.16$ & 0.30 & $2.8 \pm 0.2 \times 10^{-4}$ \\
\hline Total & & & & $1.9 \pm 1.0 \times 10^{-3}$ \\
\hline
\end{tabular}

Table 5.25: The expected number of charm particles, the trident branching ratios for each charm particle, the probability a charm event passes the selection criteria, and the resulting prior probabilities for each charm species.

the charm candidate to have a kink angle larger than $10 \mathrm{mrad}$ and a daughter momentum of greater than $1 \mathrm{GeV}$, but no longer requires the probability of a misidentified lepton. All charm prior probabilities are listed in Table 5.29.

\section{Interaction Prior Probabilities}

The interaction prior probability depends upon where the kink occurs. The interaction prior probability for tau kink candidates is

$$
\Gamma_{\text {int }}^{\mathrm{kink}}=\frac{L_{i} P_{\text {kink }} \chi}{N_{\text {total }}}
$$

where $i$ is the material in which the decay or interaction occurred. The three types of material were iron, emulsion, and plastic. $L_{i}$ is the total length traversed by all tracks in each material. $P_{\text {kink }}$ is the probability per unit length that a hadron interacts in the detector to produce a kink; this is calculated using the Monte Carlo [29] with the GEANT [38] detector simulation package. $\chi$ is the probability that a hadronic interaction event passes all of the tau criteria, which is also calculated using the Monte Carlo. The selection criteria are summarized in Sec. 4.6.3. The results are listed in Table 5.26.

The interaction prior probabilities for tau trident candidates, $\Gamma_{\text {int }}^{\text {tri }}$, are calculated using Eq. 5.9 with the probability of a hadron interacting and producing three charged particles, instead of one, per unit length and a different $\chi$. The interaction prior probabilities for tau trident candidates are summarized in Table 5.27.

Since the charm kink candidates have an identified primary lepton, the probability of selection, $\chi$, changes in the interaction prior probabilities. $\chi$ still requires the interaction candidate to have a kink angle larger than $10 \mathrm{mrad}$ and a daughter momentum of greater 
Interaction Prior Probabilities for Tau Kink Candidates

\begin{tabular}{|c|c|c|c|c|}
\hline Source & $L_{i}(\mathrm{~mm})$ & $P_{\text {kink }}$ per $\mathrm{mm}$ & $\chi$ & $\Gamma_{\text {int }}^{\mathrm{kink}}$ \\
\hline Fe Interaction & 4600 & $1.25 \times 10^{-4}$ & 0.48 & $5.1 \times 10^{-4}$ \\
\hline Emulsion Interaction & 3600 & $1.27 \times 10^{-5}$ & 0.48 & $4.1 \times 10^{-5}$ \\
\hline Plastic Interaction & 2400 & $3.49 \times 10^{-6}$ & 0.48 & $7.5 \times 10^{-6}$ \\
\hline
\end{tabular}

Table 5.26: The lengths traveled by all primary tracks, the probability of hadronic interaction producing only one charged particle per unit length, the probability that a hadronic interaction passes the tau selection criteria, and the resulting interaction prior probabilities for each type of material.

Interaction Prior Probabilities for Tau Trident Candidates

\begin{tabular}{|c|c|c|c|c|}
\hline Source & $L_{i}(\mathrm{~mm})$ & $P_{\text {trid }}$ per mm & $\chi$ & $\Gamma_{\text {int }}^{\text {tri }}$ \\
\hline Fe Interaction & 4600 & $2.2 \times 10^{-3}$ & 0.30 & $5.6 \times 10^{-3}$ \\
\hline Emulsion Interaction & 3600 & $1.0 \times 10^{-4}$ & 0.30 & $2.0 \times 10^{-4}$ \\
\hline Plastic Interaction & 2400 & $4.5 \times 10^{-5}$ & 0.30 & $6.7 \times 10^{-5}$ \\
\hline
\end{tabular}

Table 5.27: The lengths traveled by all primary tracks, the probability of hadronic interaction producing exactly three charged particles, the probability that a hadronic interaction passes the tau selection criteria, and the resulting interaction prior probabilities for each material. 
Interaction Prior Probabilities for the Charm Trident Candidate

\begin{tabular}{|c|c|c|c|c|}
\hline Source & Length $(\mathrm{mm})$ & $P_{\text {trid }}$ per mm & $P\left(\pi \rightarrow \mu \nu_{\mu}\right)$ & $\Gamma_{\text {int }}^{\text {tri }}$ \\
\hline Fe Interaction & 4600 & $2.2 \times 10^{-3}$ & 0.0195 & $3.6 \times 10^{-4}$ \\
\hline Emulsion Interaction & 3600 & $1.0 \times 10^{-4}$ & 0.0195 & $1.3 \times 10^{-5}$ \\
\hline Plastic Interaction & 2400 & $4.5 \times 10^{-5}$ & 0.0195 & $3.9 \times 10^{-6}$ \\
\hline
\end{tabular}

Table 5.28: The only charm trident candidate has an identified muon from the primary and secondary vertexes. $L_{i}$ is the length of material $i$ traversed, $P_{\text {int }}$ is the probability that a hadron interacts in material $i$ and produces three charged particles, $P(\pi \rightarrow$ trident $)$ is the probability that one of the charged particles is a $\pi$, and $P\left(\pi \rightarrow \mu \nu_{\mu}\right)$ is the probability that the $\pi$ will decay to a $\mu$ before the muon ID.

than $1 \mathrm{GeV}$, but no longer requires the probability of a misidentified lepton. All interaction prior probabilities are listed in Table 5.29.

The only charm trident candidate had an identified muon from the primary and secondary vertex. If this event were a hadronic interaction, it would have to be a muon neutrino interaction which produced a pion that decayed to a muon before the muon ID. The interaction prior probability for this scenario is

$$
\Gamma_{\text {int }}^{\text {tri }}=\frac{\sum_{i} L_{i} P_{\text {int }} P(\pi \rightarrow \text { trident }) P\left(\pi \rightarrow \mu \nu_{\mu}\right)}{N_{\text {total }}}
$$

where $L_{i}$ is the length of material $i$ traversed, $P_{\text {int }}$ is the probability that a hadron interacts in material $i$ and produces three charged particles, $P(\pi \rightarrow$ trident $)$ is the probability that one of the charged particles is a $\pi$, and $P\left(\pi \rightarrow \mu \nu_{\mu}\right)$ is the probability that the $\pi$ will decay to a $\mu$ before the muon ID. $P(\pi \rightarrow$ trident $)$ is $99 \%$, as calculated by the Monte Carlo. Table 5.28 summarizes the interaction prior probabilities for this charm trident candidate.

\section{Results for Prior Probabilities}

Table 5.29 summarizes the prior probabilities for the tau and charged charm candidates for both kink and trident events. The candidate column refers to the type of candidate, and the material column refers to where the decay occurred. The last column indicates whether the daughter was a lepton. Note the prior probabilities of the four tau candidate kink events are taken directly from [29]. 
Summary of all Prior Probabilities

\begin{tabular}{|c|c|c|c|c|c|c|c|}
\hline Event & Cand. & Topology & Material & Tau & Charm & Hadronic & $\mathrm{e} / \mu$ \\
\hline 3024_30175 & tau & kink & plastic & $4.2 \times 10^{-3}$ & $2.2 \times 10^{-4}$ & 0.00 & $\mathrm{Y}$ \\
\hline 3039_01910 & tau & kink & plastic & $1.1 \times 10^{-3}$ & $9.4 \times 10^{-4}$ & $7.4 \times 10^{-5}$ & $\mathrm{~N}$ \\
\hline 3140_22143 & tau & kink & steel & $1.6 \times 10^{-2}$ & $1.2 \times 10^{-3}$ & $5.1 \times 10^{-4}$ & $\mathrm{~N}$ \\
\hline 3263_25102 & tau & kink & steel & $1.1 \times 10^{-2}$ & $8.4 \times 10^{-4}$ & $4.9 \times 10^{-4}$ & $\mathrm{~N}$ \\
\hline $3296 \_18816$ & tau & trident & emulsion & $2.7 \times 10^{-3}$ & $1.9 \times 10^{-3}$ & $2.0 \times 10^{-4}$ & $\mathrm{~N}$ \\
\hline 3333_17665 & tau & kink & plastic & $4.2 \times 10^{-3}$ & $1.0 \times 10^{-4}$ & 0.00 & $\mathrm{Y}$ \\
\hline 3334_19920 & tau & trident & emulsion & $2.7 \times 10^{-3}$ & $1.9 \times 10^{-3}$ & $2.0 \times 10^{-3}$ & $\mathrm{~N}$ \\
\hline 2846_09042 & charm & kink & steel & 0.00 & $4.0 \times 10^{-3}$ & $1.7 \times 10^{-3}$ & $\mathrm{~N}$ \\
\hline 3065_03238 & charm & kink & steel & 0.00 & $4.0 \times 10^{-3}$ & $1.7 \times 10^{-3}$ & $\mathrm{~N}$ \\
\hline 3227_03420 & charm & kink & plastic & 0.00 & $4.0 \times 10^{-3}$ & $2.5 \times 10^{-5}$ & $\mathrm{~N}$ \\
\hline 3245_22786 & charm & trident & emulsion & 0.00 & $6.4 \times 10^{-3}$ & $1.3 \times 10^{-5}$ & $\mathrm{Y}$ \\
\hline
\end{tabular}

Table 5.29: The prior probabilities for the all tau and charged charm candidates. The candidate column refers to the type of candidate, and the material column refers to where the decay occurs. The last column indicates whether the daughter was a lepton.

\subsubsection{Probability Density}

The probability density was calculated using simulated interactions. The neutrino-nucleon interactions were simulated using the LEPTO event generator [37], which provided a list of simulated tau leptons from tau neutrino charged-current interactions and charm particles from electron or muon neutrino charged-current interactions. The hadronic interactions were simulated using the GEISHA hadronic interaction simulator [36]. The particles were propagated through the detector using the GEANT detector simulation [38]. The Monte Carlo provided weights for each event; the weights were the product of the production and interaction probabilities.

The probability density function evaluated at $\{x\}$ is

$$
\Pi(\{x\} \mid i)=\frac{W_{\Delta v}}{W_{\text {total }} \Delta v}
$$

where $\Delta v$ is a region in parameter space centered around $\{x\}, W_{\Delta v}$ is the weight of all events which reside in $\Delta v$, and $W_{\text {total }}$ is the total weight of all events.

The parameters used in this analysis and their simulated distributions were described in Sec. 4.5 ; they are $\theta$, the production angle, $\Delta \phi$, the polar angle asymmetry, $L$, the decay length, $\alpha$, the kink angle, $P_{\mathrm{d}}$, the momentum of the daughter, and $\Sigma I P$, the sum of the 
The Five Parameters used in the Kink Analysis

\begin{tabular}{|c|c|c|c|c|c|c|}
\hline Type & Event & $\theta(\mathrm{rad})$ & $\Delta \Phi(\mathrm{rad})$ & $\mathrm{L}(\mathrm{mm})$ & $\alpha(\mathrm{rad})$ & $P_{\mathrm{d}}(\mathrm{GeV})$ \\
\hline$\tau$ & $3024 \_30175$ & 0.028 & 1.09 & 4.59 & 0.093 & $2.9_{-0.7}^{+1.5}$ \\
\hline$\tau$ & $3039 \_01910$ & 0.067 & 2.71 & 0.29 & 0.090 & $4.6_{-0.4}^{+1.4}$ \\
\hline$\tau$ & $3263 \_25102$ & 0.169 & 0.10 & 1.97 & 0.130 & $1.9_{-0.7}^{+2.2}$ \\
\hline$\tau$ & $3140 \_22143$ & 0.040 & 1.67 & 4.8 & 0.012 & $13.4_{-3.7}^{+7.0}$ \\
\hline$\tau$ & $3333 \_17665$ & 0.016 & 2.84 & 0.55 & 0.013 & $21.4_{-0.6}^{+14}$ \\
\hline charm & 2846_09042 & 0.034 & 2.81 & 7.0 & 0.165 & $26_{-9.0}^{+21}$ \\
\hline charm & $3227 \_03420$ & 0.010 & 1.90 & 1.8 & 0.070 & $4.5_{-1.4}^{+3.4}$ \\
\hline charm & 3065_03238 & 0.250 & 0.71 & 2.10 & 0.229 & $1.2_{-0.3}^{+0.4}$ \\
\hline
\end{tabular}

Table 5.30: $\theta$ is the production angle, $\Delta \phi$ is the polar angle imbalance, $L$ is the decay length, $\alpha$ is the kink angle, and $P_{\mathrm{d}}$ is the daughter momentum. The average uncertainty in $\theta$ is $1.5 \mathrm{mrad}$; the average uncertainty in $\Delta \phi$ is $9 \mathrm{mrad}$; the average uncertainty in $L$ is 10 microns; the maximum uncertainty in $\alpha$ is less than $1 \mathrm{mrad}$. [29]

daughter impact parameters. The values of these parameters for each candidate are listed in Table 5.30 and 5.31.

In this section, the one-dimensional projections are used for illustrative purposes. These distributions can be misleading as the analysis actually uses an N-dimensional parameter space. For example, suppose we look at two parameters, the kink angle and the momentum distribution. In Fig. 5.53 and 5.54, the five tau kink candidates's values are represented by black lines. By looking at these one-dimensional distributions individually, these events could be hadronic interactions or tau events. Now we can look at the

The Four Parameters used in the Trident Analysis

\begin{tabular}{|c|c|c|c|c|c|}
\hline Type & Event & $\theta(\mathrm{rad})$ & $\Delta \phi(\mathrm{rad})$ & $\mathrm{L}(\mathrm{mm})$ & $\Sigma$ IP $(\mathrm{mm})$ \\
\hline$\tau$ & $3334 \_19920$ & 0.040 & 3.11 & 8.87 & 0.0363 \\
\hline$\tau$ & $3296 \_18816$ & 0.141 & 1.74 & 0.78 & 0.0293 \\
\hline charm & $3245 \_22786$ & 0.142 & 0.16 & 0.40 & 0.0128 \\
\hline
\end{tabular}

Table 5.31: $\theta$ is the production angle, $\Delta \phi$ is the polar angle imbalance, $L$ is the decay length, and $\Sigma I P$ is the sum of the daughters's impact parameters. The average uncertainty in $\theta$ is $1.5 \mathrm{mrad}$; the average uncertainty in $\Delta \phi$ is $9 \mathrm{mrad}$; the average uncertainty in $L$ is 10 microns; the average uncertainty in $\Sigma I P$ is dominated by the uncertainty in the decay length, which is 10 microns. 
Summary of all Probability Densities

\begin{tabular}{|c|c|c|c|c|c|}
\hline Event & Candidate & Topology & Tau & Charm & Interaction \\
\hline 3024_30175 & tau & kink & 0.17 & 2.0 & - \\
\hline 3039_01910 & tau & kink & 16 & 7.8 & 0.22 \\
\hline $3140 \_22143$ & tau & kink & 9.2 & 3.5 & 1.4 \\
\hline $3263 \_25102$ & tau & kink & 0.01 & 0.27 & 0.90 \\
\hline 3296_18816 & tau & trident & 22 & 13 & 7.4 \\
\hline 3333_17665 & tau & kink & 14 & 2.4 & - \\
\hline 3334_19920 & tau & trident & 404 & 1.2 & 2.5 \\
\hline 2846_09042 & charm & kink & 590 & 735 & 270 \\
\hline 3065_03238 & charm & kink & 0.32 & 2.0 & 0.45 \\
\hline 3227_03420 & charm & kink & 59 & 74 & 27 \\
\hline 3245_22786 & charm & trident & 32 & 222 & 210 \\
\hline
\end{tabular}

Table 5.32: The probability density in $(\mathrm{mm} \mathrm{GeV})^{-1}$ for the kink candidates and $(\mathrm{mm})^{-2}$ for the trident candidates.

transverse momentum, $P_{t}$ where $P_{t}=P_{\mathrm{d}} \sin \alpha$. Fig. 5.55 and 5.56 show the transverse momentum distributions for tau events and hadronic interactions with these same five events represented. These events now look very likely to be tau events, and very unlikely to be hadronic interactions. The $P_{t}$ distribution for tau events was plotted on a linear scale, while the $P_{t}$ distribution for the interaction event was plotted on a log scale. As these parameters are used with additional parameters, they provide a more discriminating analysis than just looking at the one-dimensional distributions.

The one-dimensional distributions for the remaining four parameters are shown in Fig. 5.57, 5.58, 5.59, and 5.60. Each candidates's parameters are represented by black lines in the distributions.

The probability densities for each tau and charged charm candidate are calculated using Eq. 5.11 and the results are listed in Table 5.32. The two events which have no probability density have an interaction prior probability of zero; therefore, the probability density for the interaction event type is unnecessary. 


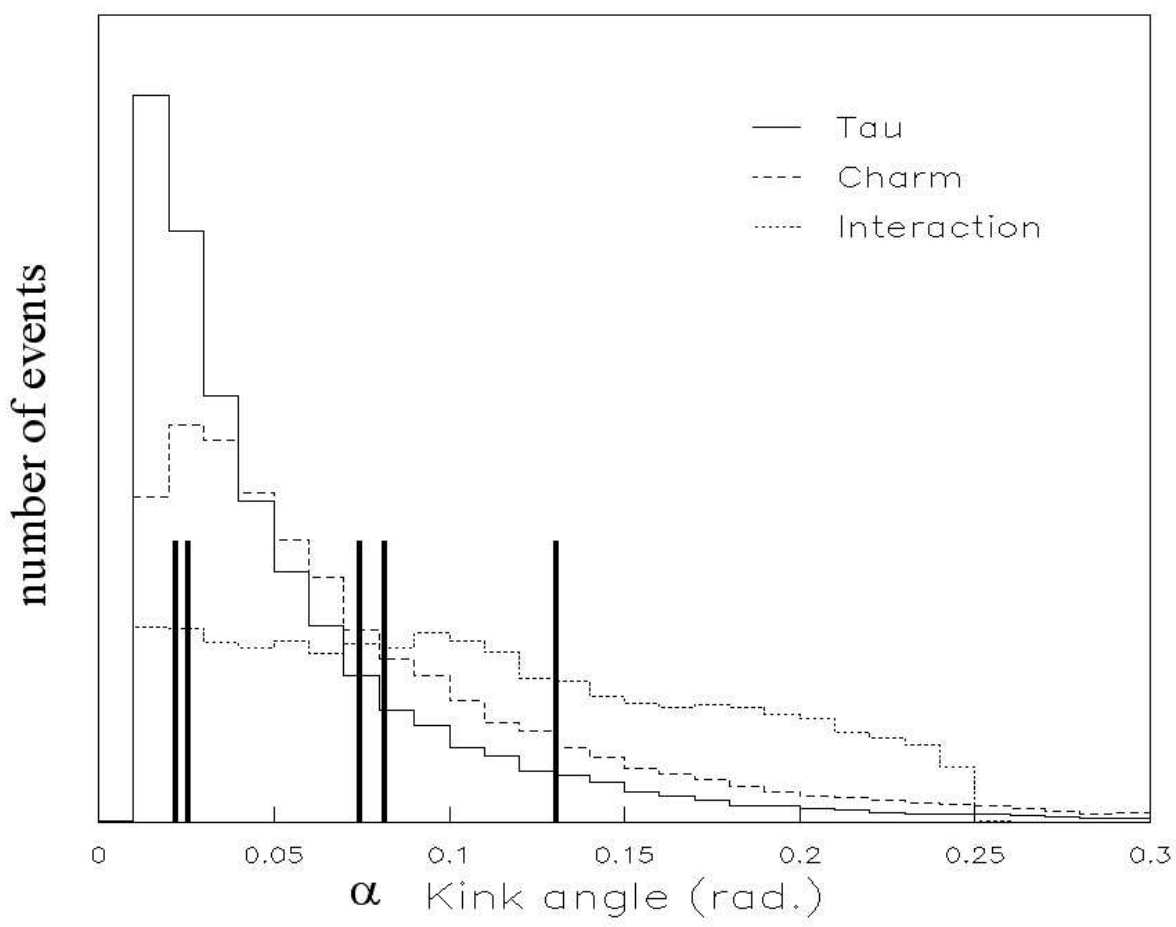

Figure 5.53: Distributions of $\alpha$ for simulated tau, charm, and interaction events. The kink angle provided significant distinction between the tau and interaction events. Large kinks are more probable for interaction events than for tau events because there are many more low momentum hadrons. The value of $\alpha$ for each of the kink candidate events is represented by a dark line in the distribution above. According to this distribution, three of the five events could be hadronic interactions or tau events. 


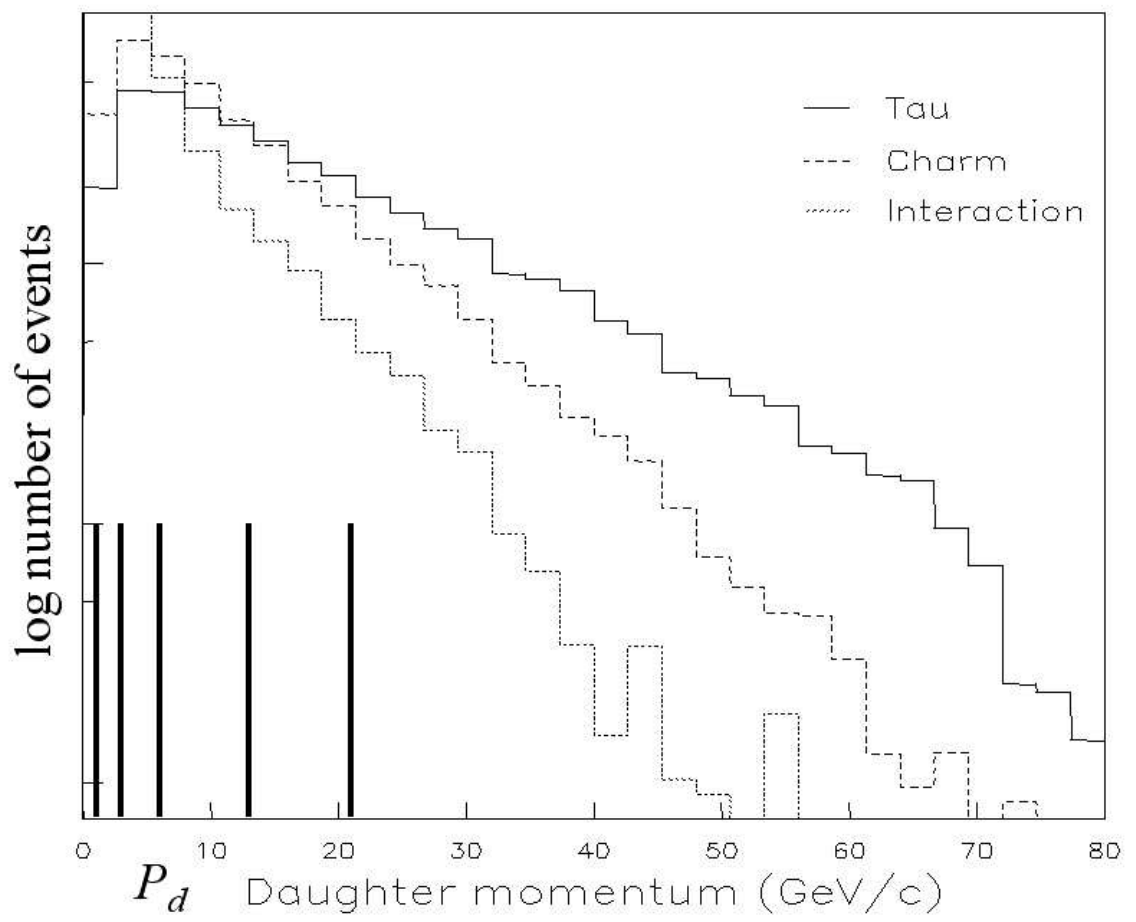

Figure 5.54: Distributions of $P_{\mathrm{d}}$ for simulated tau, charm, and interaction events. The distribution shown above does not include the three prong decays. The daughter momentum provided a distinction between the tau events and the interaction backgrounds. The daughter momenta of the five kink events are represented by black lines in the distribution. According to this distribution, three of these events are likely to be tau neutrino interactions or interaction backgrounds. 


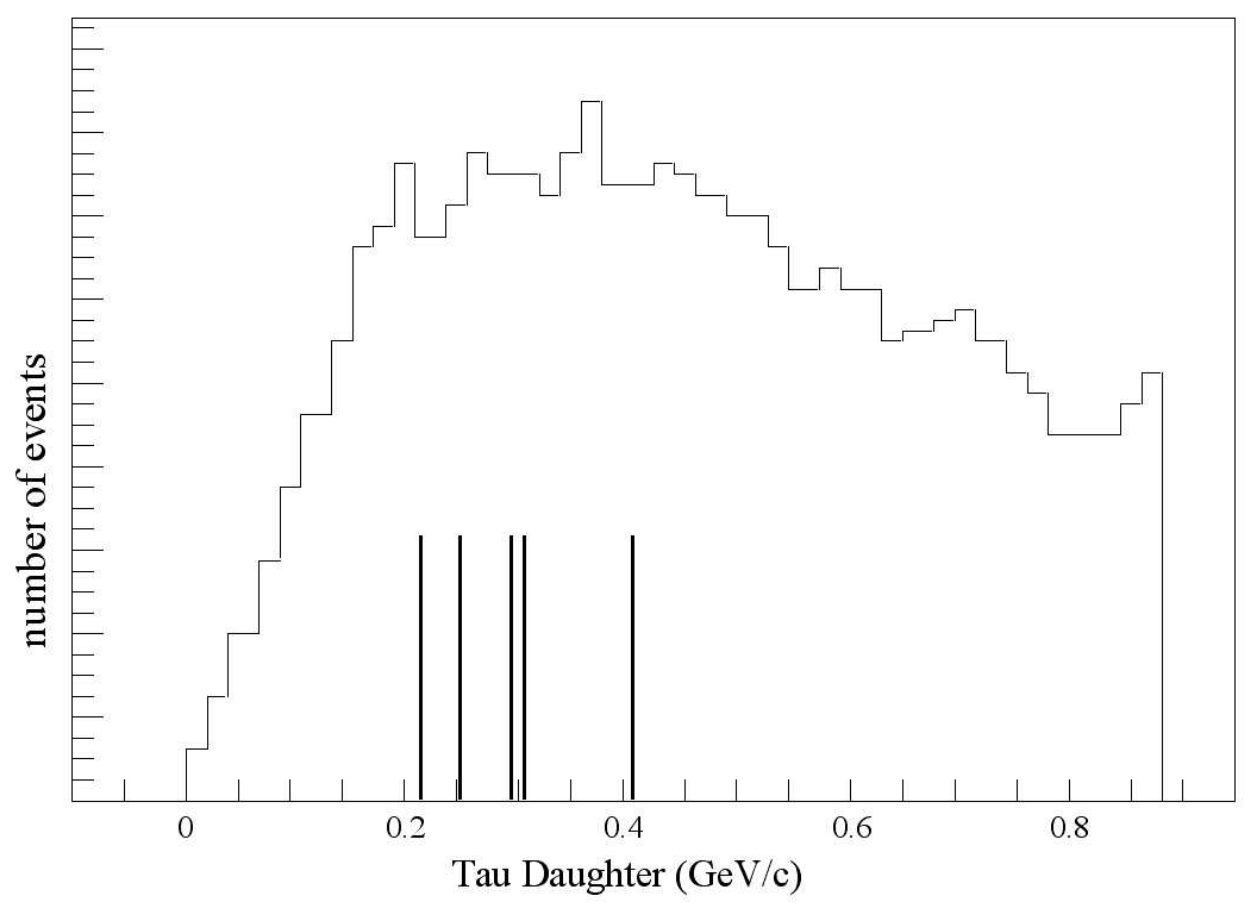

Figure 5.55: Distributions of $P_{t}$ for simulated tau events. The transverse momentum combines the kink angle and the daughter momentum. The $P_{t}$ of each kink candidate is represented by a black line in the distribution above. According to this parameter, the five kink candidates look likely to be tau events compared to hadronic interactions, as shown in Fig. 5.56. 


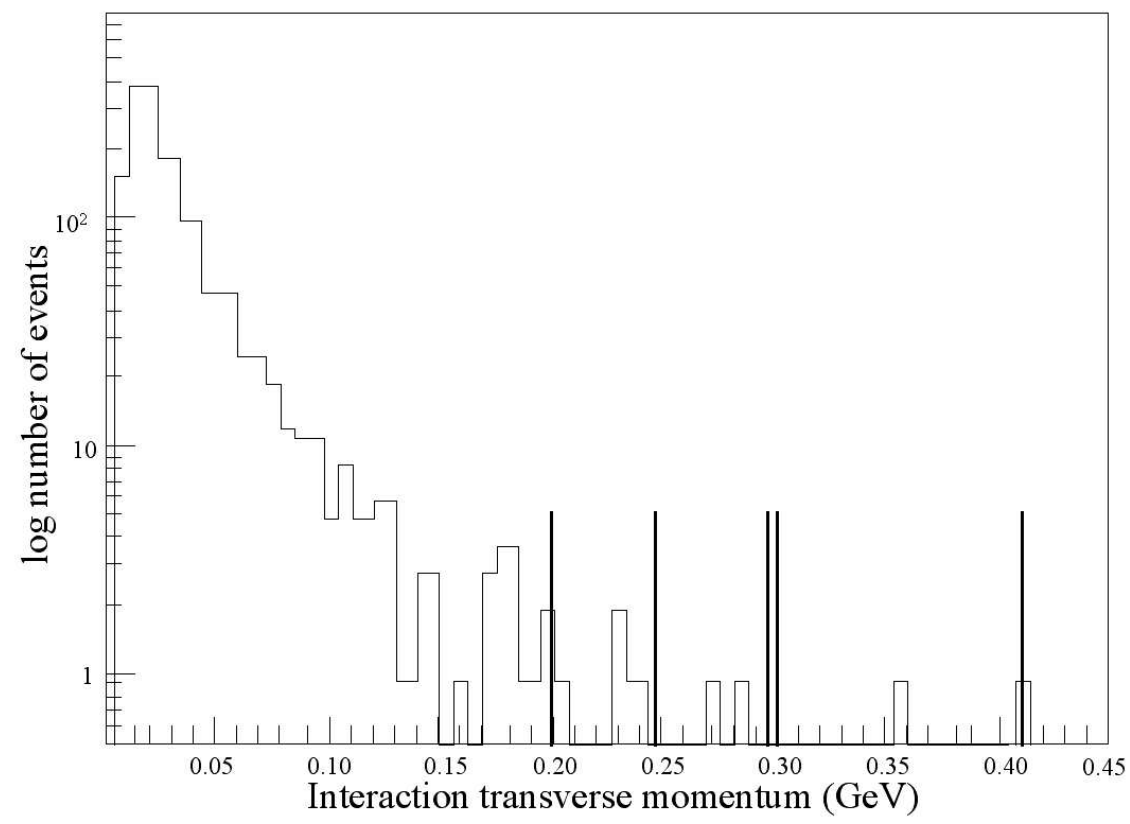

Figure 5.56: Distributions of $P_{t}$ for simulated interaction events plotted on a log scale. The transverse momentum combines the kink angle and the daughter momentum. The $P_{t}$ of each kink candidate is represented by a black line in the distribution above. According to this parameter, it is unlikely that any of the five events are hadrons. 


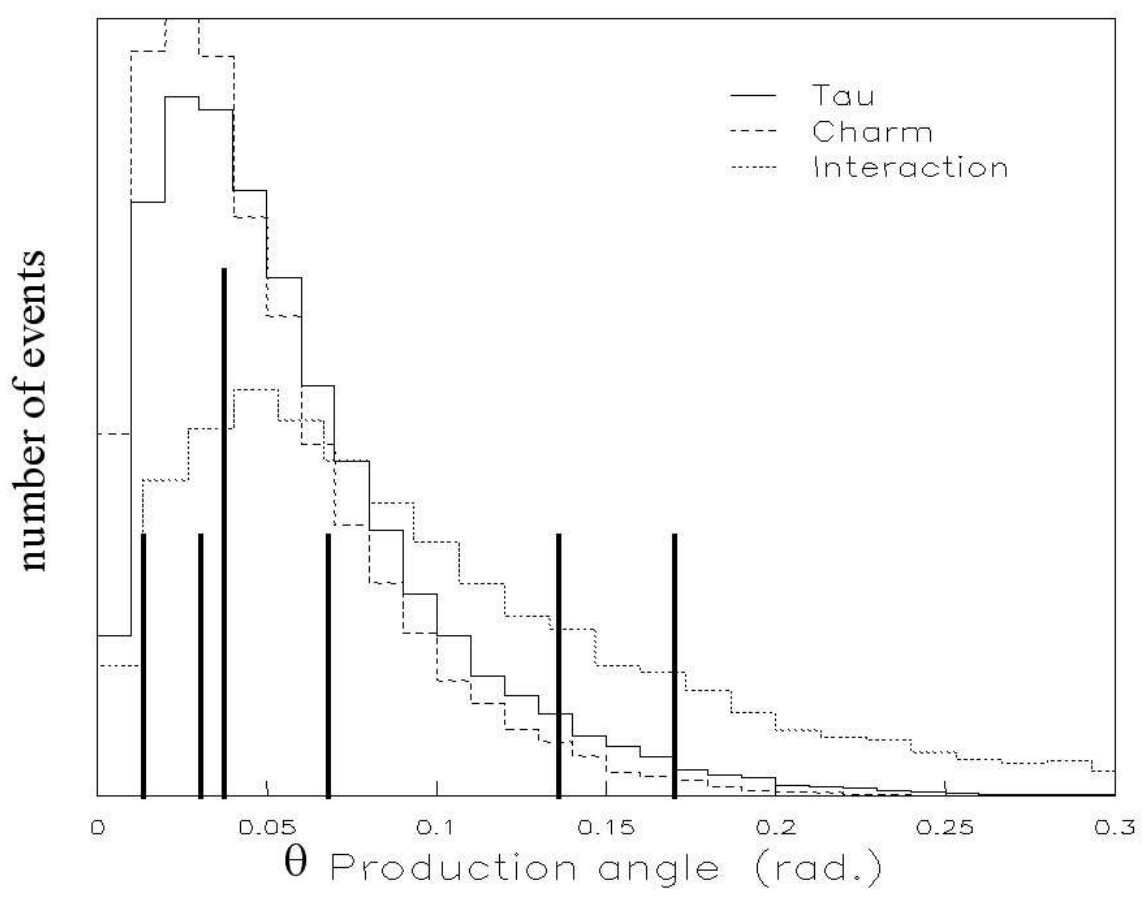

Figure 5.57: Distributions of $\theta$ for simulated tau, charm, and interaction events. This parameter provided significant distinction between the tau and hadrons events. The production of tau leptons in high energy tau neutrino interactions are mainly in the forward direction; therefore, the tau distribution is peaked near zero. The distributions were normalized using the Monte Carlo weight of each event. The weight is the product of the probability of production and interaction. The value of $\theta$ for each of the candidates is represented by a dark line in the distribution above. According to this distribution two of the seven candidates appear more like interaction background events than tau events. This distribution, however, is not used in the analysis. The distribution is integrated over all other parameters and is only used for illustrative purposes. The actual analysis uses uses an N-dimensional distribution to calculate the probability density. 


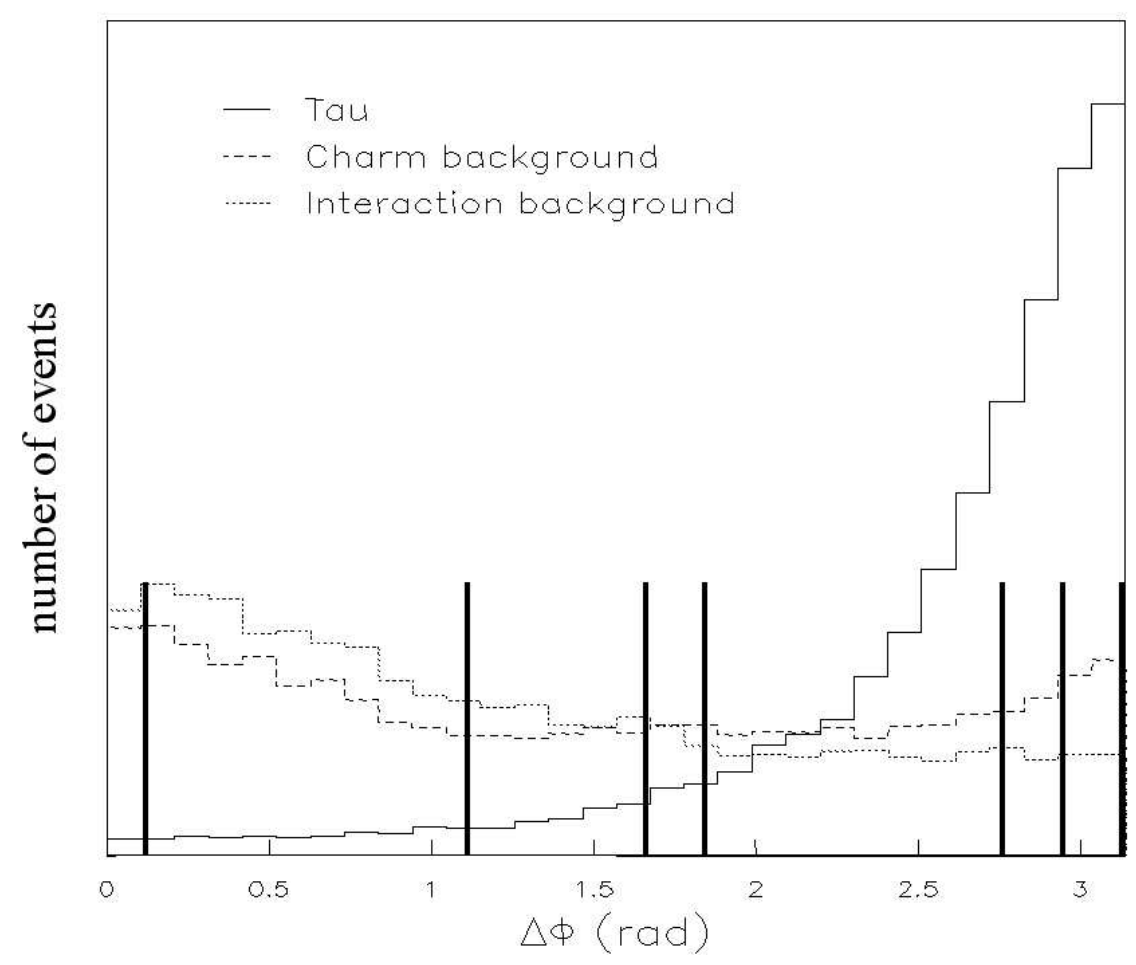

Figure 5.58: Distributions of $\Delta \Phi$ for simulated tau, charm, and interaction events. The polar angle is a measure of the transverse momentum balance. The particles which produce background kinks are not constrained to this momentum balance since the kinks are not caused by the lepton from the neutrino interaction. For background events, $\Delta \phi$ is the result of random distribution of primary tracks; therefore, they are not peaked at $\pi$ like the taus. The polar angle provided significant distinction between the charm and interaction background and the tau events. The value of $\Delta \phi$ for each of the candidate events is represented by a dark line in the distribution above. According to this distribution four of the seven candidates appear more like interaction or charm background events than tau events. This distribution, however, is not used in the analysis. The distribution is integrated over all other parameters and is only used for illustrative purposes. The actual analysis uses uses an N-dimensional distribution to calculate the probability density. 


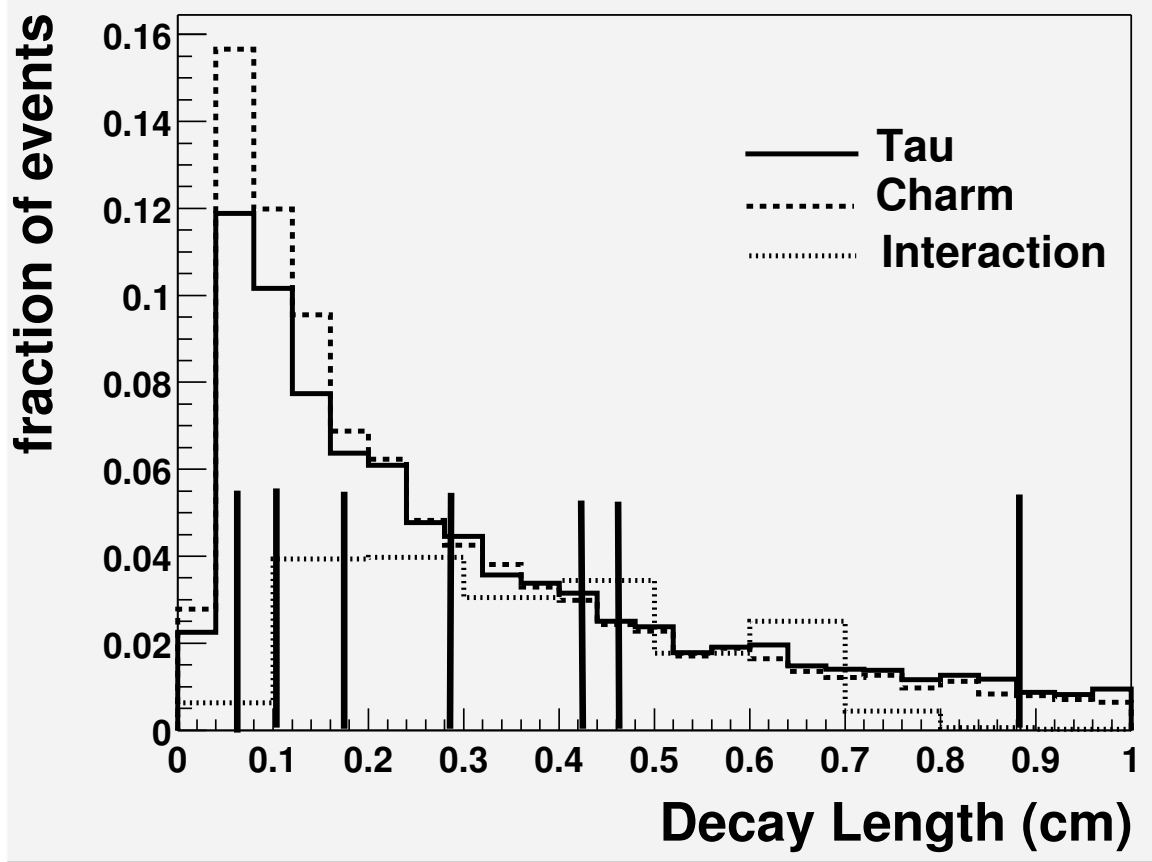

Figure 5.59: Distributions of $L$ for simulated tau, charm, and interaction events. This parameter provided distinction between the interaction backgrounds and the tau events. The tau and charm distributions are similar; they are exponentials and are strongly peaked at zero. The value of $L$ for each candidate event is represented by a dark line in the distribution above. According to this distribution three of the seven candidates are as likely to be interaction or charm background events as they are to be tau events. This distribution, however, is not used in the analysis. The distribution is integrated over all other parameters and is only used for illustrative purposes. The actual analysis uses uses an $\mathrm{N}$-dimensional distribution to calculate the probability density. 


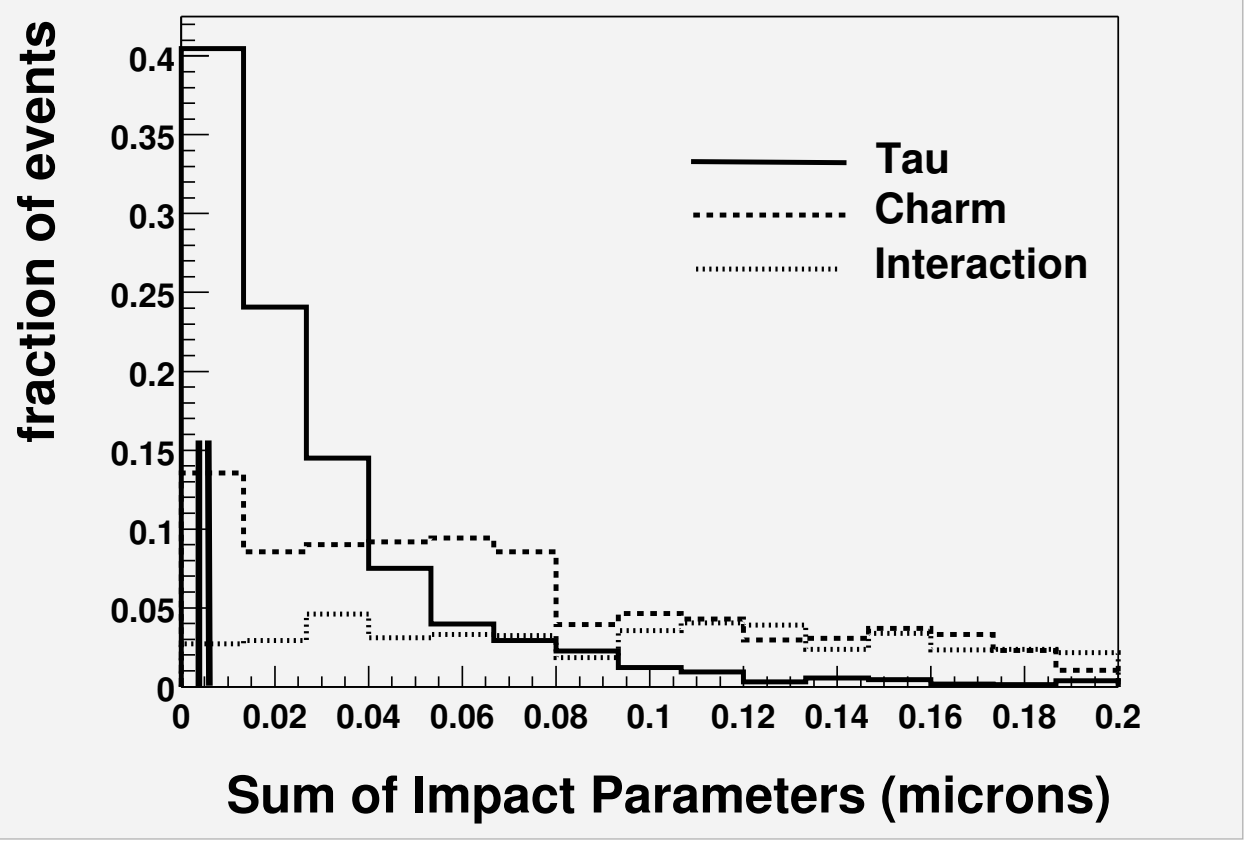

Figure 5.60: Distributions of $\Sigma I P$ for simulated tau, charm, and interaction events. The sum of the impact parameters was closely related to the kink angles of the daughters and the length of the tau lepton track. Since the tau lepton track was generally shorter than the primary track from a hadronic interaction, the tau distribution peaked at a lower values. The value of $\Sigma I P$ for each of the trident candidate events is represented by a dark line in the distribution above. According to this distribution the two trident candidates are most likely tau events. This distribution, however, is not used in the analysis. The distribution is integrated over all other parameters and is only used for illustrative purposes. The actual analysis uses uses an N-dimensional distribution to calculate the probability density. 
Relative Probabilities for Each Candidate

\begin{tabular}{|c|c|c|c|c|c|c|}
\hline Event & Material & Topology & Tau & Charm & Interaction & Overall \\
\hline 3024_30175 & plastic & kink & 0.64 & 0.36 & 0.00 & tau \\
\hline $3039 \_01910$ & plastic & kink & 0.96 & 0.04 & 0.00 & tau \\
\hline $3140 \_22143$ & steel & kink & 0.97 & 0.03 & 0.00 & tau \\
\hline $3263 \_25102$ & steel & kink & 0.16 & 0.29 & 0.57 & interaction \\
\hline $3296 \_18816$ & emulsion & trident & 0.71 & 0.29 & 0.00 & tau \\
\hline $3333 \_17665$ & plastic & kink & 0.99 & 0.01 & 0.00 & tau \\
\hline $3334 \_19920$ & plastic & trident & 1.0 & 0.00 & 0.00 & tau \\
\hline $2846 \_09042$ & steel & kink & 0.00 & 0.87 & 0.13 & charm \\
\hline 3065_03238 & steel & kink & 0.00 & 0.91 & 0.09 & charm \\
\hline $3227 \_03420$ & plastic & kink & 0.00 & 0.99 & 0.01 & charm \\
\hline $3245 \_22786$ & emulsion & trident & 0.00 & 0.99 & 0.01 & charm \\
\hline
\end{tabular}

Table 5.33: The relative probabilities summarized in the table above are the results of a statistical analysis, calculated using Bayes's theorem. Six of the tau candidates are likely to be tau neutrino charged-current interactions according to this analysis. One is more likely a hadronic interaction. All charm candidates are likely to be a charged-current muon or electron neutrino interaction which produced a charm that decayed.

\subsubsection{Results}

Table 5.33 lists the results, which are relative probabilities of each hypothesis for each of the tau and charged charm candidates. These were calculated using Eq. 5.1, Table 5.29, and Table 5.32. According to this analysis one of the tau candidates is most likely a hadronic interaction. The remaining six candidates are likely to be tau neutrino chargedcurrent interactions. All charm candidates are likely to be a charged-current muon or electron neutrino interaction which produced a charm that decayed.

\subsubsection{Testing the Statistical Analysis}

A test of the statistical analysis was performed using the charm candidates. Each charm candidate has an identified charged lepton from the primary; therefore, the relative tau probability is zero. If the primary lepton was missed, the relative probabilities could shift, but the set of tau candidates should not change. The statistical analysis was performed on a subset of the charm candidates assuming the lepton was missed. The results are summarized in Table 5.34. While the relative probabilities did shift, the events would still 
Results of a test of the Statistical Analysis

\begin{tabular}{|c|c|c|c|c|c|}
\hline Event & Material & Topology & Tau & Charm & Interaction \\
\hline $3065 \_03238$ & steel & kink & 0.43 & 0.53 & 0.05 \\
\hline $3227 \_03420$ & plastic & kink & 0.48 & 0.50 & 0.02 \\
\hline 3245_22786 & emulsion & trident & 0.11 & 0.24 & 0.64 \\
\hline
\end{tabular}

Table 5.34: The relative probabilities for a set of charm candidates, assuming the primary lepton was missed. The resulting set of tau events remained unchanged.

not be classified as tau events. Thus the set of tau candidates remains unchanged even if the primary lepton is missed. 


\section{Chapter 6}

\section{Cross Section Analysis}

This chapter presents the measurements of the tau neutrino charge-current interaction cross section in the deep inelastic scattering region. Two techniques were used to measure the cross section. For the primary measurement, the tau neutrino cross section was measured relative to the electron and muon neutrino cross sections; systematic errors which affected all neutrino interactions equally canceled in the relative measurements. Since the muon neutrinos were produced in both charm and light meson decays, the fraction of prompt to non-prompt muon neutrino interactions was required for the measurement of the tau neutrino cross section relative to the muon neutrino cross section. This fraction was measured in [28]. Since the prompt fraction had an associated uncertainty, this measurement had a larger overall uncertainty. The second technique measured the absolute cross section of the tau neutrino; this result provided a check of the relative measurements. The techniques used to make these measurements were also used to measure the absolute electron and muon cross sections and measure the electron neutrino cross section relative to the muon neutrino cross section. Since the electron and muon neutrino cross sections are well known, the measured and actual values were compared to test the validity of the technique.

Lepton universality, which is a feature of the standard model, dictates that neutrinos interact identically, aside from the differences in the kinematics of their interactions. If these differences are taken into account, the cross sections of the neutrinos should be equal. The analysis in this thesis was designed to test this predicted equality, and, therefore, lepton universality. In addition to lepton universality, the standard model predicts the tau neutrino cross section; this prediction was given in Ch. 2. All results were compared to the standard model predictions. 


\subsection{Cross Section Formula}

The observable used to determine the cross section was the observed number of events in the data, $N_{\nu_{\alpha}}^{\text {obs }}$, which is given by

$$
N_{\nu_{\alpha}}^{\mathrm{obs}}=R_{\nu_{\alpha}}(E) \times N_{\text {pot }} \times \epsilon_{\nu_{\alpha}}
$$

where $R_{\nu_{\alpha}}(E)$ is the rate of $\alpha$-type neutrino interactions per proton on target, $N_{\text {pot }}$ is the

number of protons on target, and $\epsilon_{\nu_{\alpha}}$ is the efficiency of locating and identifying an $\alpha$-type neutrino interaction. The rate of neutrino interactions per proton on target is

$$
R_{\nu_{\alpha}}(E)=\int N_{\nu_{\alpha}}^{\operatorname{tar}}(E) \times \sigma_{\nu_{\alpha} N}(E) \times N_{\text {scat }} d E
$$

where $N_{\nu_{\alpha}}^{\operatorname{tar}}(E)$ is the number of neutrinos incident on the target per proton, $\sigma_{\nu_{\alpha} N}(E)$ is the cross section for the charged-current interaction of an $\alpha$-type neutrino and a nucleon, and $N_{\text {scat }}$ is the number of scattering centers per $\mathrm{cm}^{2}$.

The number of observed events, $N_{\nu \alpha}^{\mathrm{obs}}$, and the number of protons on target, $N_{\text {pot }}$, were measured from the data. The number of scatterers, $N_{\text {scat }}$, was calculated, as described in Sec. 6.2.7. The number of neutrinos incident on the target per proton and the efficiencies were calculated using the Monte Carlo, which was described in Sec. 4.4.

In a relative measurement of the tau neutrino charged current cross section to the electron neutrino charged-current cross section,

$$
\begin{aligned}
\frac{N_{\nu_{\tau}}^{\text {obs }}}{N_{\nu_{e}}^{\text {obs }}} & =\frac{N_{\text {scat }} \times N_{\text {pot }} \times \int N_{\nu_{\tau}}^{\operatorname{tar}}(E) \times \epsilon_{\nu_{\tau}}(E) \times \sigma_{\nu_{\tau} N}(E) d E}{N_{\text {scat }} \times N_{\text {pot }} \times \int N_{\nu_{e}}^{\operatorname{tar}}(E) \times \epsilon_{\nu_{e}}(E) \times \sigma_{\nu_{e} N}(E) d E} \\
& =\frac{\int N_{\nu_{\tau}}^{\operatorname{tar}}(E) \times \epsilon_{\nu_{\tau}}(E) \times \sigma_{\nu_{\tau} N}(E) d E}{\int N_{\nu_{e}}^{\operatorname{tar}}(E) \times \epsilon_{\nu_{e}}(E) \times \sigma_{\nu_{e} N}(E) d E} .
\end{aligned}
$$

$N_{\text {scat }}$ and $N_{\text {pot }}$ were functions of the target and thus cancel.

At these energies, the electron and muon neutrino cross sections are linear in energy [25], and, therefore, the cross section is

$$
\sigma_{\nu_{e} N}(E)=E_{\nu_{e}} \times \sigma_{\nu_{e} N}^{\text {const }}
$$

where $\sigma_{\nu_{e} N}^{\text {const }}$ is the constant part of the electron neutrino cross section, and $E_{\nu_{e}}$ is the energy of the electron neutrinos. From lepton universality, the tau neutrino cross section should be equal to the electron neutrino cross section, except for the kinematic differences.

$$
\sigma_{\nu_{\tau} N}(E)=K_{F}(E) \times \sigma_{\nu_{e} N}(E)
$$


where $K_{F}(E)$ accounts for the kinematic differences in the electron and tau chargedcurrent neutrino interactions. Eq. 6.4 and 6.5 are combined, and

$$
\sigma_{\nu_{\tau} N}(E)=K_{F}(E) \times E_{\nu_{\tau}} \times \sigma_{\nu_{\tau} N}^{\text {const }}
$$

Combining Eq. 6.4, Eq. 6.4, and Eq. 6.6 yields

$$
\frac{N_{\nu_{\tau}}^{\mathrm{obs}}}{N_{\nu_{e}}^{\mathrm{obs}}}=\frac{\int N_{\nu_{\tau}}^{\mathrm{tar}}(E) \times \epsilon_{\nu_{\tau}}(E) \times K_{F}(E) \times E_{\nu_{\tau}} \times \sigma_{\nu_{\tau} N}^{\text {const }} d E}{\int N_{\nu_{e}}^{\mathrm{tar}}(E) \times \epsilon_{\nu_{e}}(E) \times E_{\nu_{e}} \times \sigma_{\nu_{e} N}^{\text {const }} d E} .
$$

Simplifying this equation yields

$$
\frac{N_{\nu_{\tau}}^{\text {obs }}}{N_{\nu_{e}}^{\text {obs }}}=\frac{\sigma_{\nu_{\tau} N}^{\text {const }} \times \int N_{\nu_{\tau}}^{\operatorname{tar}}(E) \times \epsilon_{\nu_{\tau}}(E) \times K_{F}(E) \times E_{\nu_{\tau}} d E}{\sigma_{\nu_{e} N}^{\text {const }} \times \int N_{\nu_{e}}^{\operatorname{tar}}(E) \times \epsilon_{\nu_{e}}(E) \times E_{\nu_{e}} d E} .
$$

Solving for the ratio of the constant part of the tau neutrino cross section to the constant part of the electron neutrino cross section, $\frac{\sigma_{\nu_{\tau}}^{\text {const }}}{\sigma_{\nu_{e} N}^{\text {const }}}$, yields

$$
\frac{\sigma_{\nu_{\tau} N}^{\text {const }}}{\sigma_{\nu_{e} N}^{\text {const }}}=\frac{N_{\nu_{\tau}}^{\mathrm{obs}} \times \int N_{\nu_{e}}^{\mathrm{tar}}(E) \times \epsilon_{\nu_{e}}(E) \times E_{\nu_{e}} d E}{N_{\nu_{e}}^{\mathrm{obs}} \times \int N_{\nu_{\tau}}^{\mathrm{tar}}(E) \times \epsilon_{\nu_{\tau}}(E) \times K_{F}(E) \times E_{\nu_{\tau}} d E} .
$$

\subsection{Quantities for Cross Section Calculation}

In this section, each of the quantities in Eq. 6.9 is discussed. The absolute measurement requires the value for each quantity, while the relative measurement only requires ratios.

\subsubsection{The Number of Observed Tau Neutrino Interaction}

After applying the event classification requirements, seven tau candidates remained. A further statistical analysis, discussed in Sec. 5.3, found that one of the candidate was more likely a hadronic interaction. This left six identified tau neutrino interactions. For the cross section, only deep inelastic scattering interactions were considered. In Appendix A, one of the tau neutrino candidates was shown to reside outside of the deep inelastic scattering region. This left five observed tau neutrino interactions in the final set used to measure the cross section in the deep inelastic scattering region.

\subsubsection{Efficiencies}

The efficiencies of four distinct event types needed consideration: the tau neutrino chargedcurrent interaction with a single-prong tau lepton decay or a trident tau lepton decay, the 
charged-current interaction of the electron neutrino, and the charged-current interaction of the muon neutrino. Each efficiency was separated into the trigger, selection, and identification efficiencies and explained in this section. Each efficiency was weakly energy dependent. The dependence was strongest at low energies. Since this cross section is only relevant in the deep inelastic scattering region, this low-energy dependence did not significantly affect the cross section. All efficiencies were measured using the Monte Carlo. The trigger efficiency is the fraction of neutrino interactions recorded by the spectrometer; the trigger criteria was discussed in Sec. 4.2.1. The selection efficiency is the fraction of neutrino interactions which passed the software and visual criteria, as described in Sec. 4.2.2 and Sec. 4.2.3.

The electron identification efficiency is the fraction of electron neutrino charged-current interaction with an identified primary electron. The electron is identified in the emulsion, the scintillating fibers, or the EMCAL. Electron-positron pairs, produced by the electron as it traveled through the emulsion, were used to identify electrons with an efficiency of $51 \%$. In the spectrometer, electrons are identified through their electromagnetic showers. Since hadrons also shower, the electron identification must differentiatie between electromagentic and hadronic showers. The shower shape is different for these two types of showers; thus, the showers were often visually differentiated. Hadronic showers tend to be more laterally spread out than electromagnetic ones. When uncertiatity existed, a neural net analysis, which used spectometer data, was used to classify the shower as electromagnetic or hadronic [41]. The neutral net analysis used the number of continuous blocks with deposited energy in the EMCAL, the average energy depositied in these blocks, the angles of the tracks which deposited the energy, and the number of hits and pulse heights in the scintillating fibers. Using only the scintillating fibers, $62 \%$ of electron were identified, where approxiametly $8 \%$ were hadrons misidentifed as electrons. Using the EMCAL $51 \%$ of electrons were identified, with a hadronic contatmination rate of $6 \%$. Using the emulsion, scintilalting fibers, and EMCAL, the overall electron identification efficiceny was $72 \%$ with a contamination of $5 \%$ hadrons [46].

The identification of muon neutrino charged-current interactions hinged on identifying the muon produced in the interaction; the muons were identified using the muon ID in the spectrometer. Each possible muon track was projected to each muon ID wall. A muon track was required to have at least four out of six possible hits, with one hit in each of the three muon ID walls. If one of the primary tracks was identified as a muon, the event was classified as a muon neutrino charged-current interaction. If a muon passes through the muon ID, the efficiency of identification was $97 \%$. However, the areas of the muon 


\section{Efficiencies of Kink Selection Criteria}

\begin{tabular}{|l|l|l|}
\hline Criterion & Cut & Efficiency (\%) \\
\hline Decay Length & must have $\geq 1$ emulsion hit & 76 \\
\hline Decay Angle & greater than $10 \mathrm{mrad}$ & 84 \\
\hline Daughter IP & less than $500 \mu \mathrm{rad}$ & 97 \\
\hline Daughter P & greater than $1 \mathrm{GeV} / \mathrm{c}$ & 96 \\
\hline Total & & 60 \\
\hline
\end{tabular}

Table 6.1: The efficiency of the selection criteria required to identify the tau neutrino kink interaction, which were calculated using the Monte Carlo. [29]

\section{Efficiencies of Trident Selection Criteria}

\begin{tabular}{|l|l|l|}
\hline Criterion & Cut & Efficiency (\%) \\
\hline Tau Track & must have $\geq 1$ emulsion hit & 76 \\
\hline Total & & 76 \\
\hline
\end{tabular}

Table 6.2: The efficiency of the selection criteria required to identify the tau neutrino trident interaction, which was calculated using the Monte Carlo.

ID that were in the path of the high density muon plumes were not used because of the high track density. Thus, the acceptance of the muon ID was 73\% [29] for prompt muon neutrino charged-current interactions.

The tau neutrino charged-current interactions were classified by identifying the tau lepton and the subsequent decay to a single or three charged particles. The requirements for kink and trident decays were explained in Sec. 4.3.3. Each selection criteria had a certain efficiency, found by simulating tau lepton decays and calculating the fraction which passed each cut. These efficiencies are summarized in Table 6.1 for the kink events and in Table 6.2 for the trident events. The total efficiency of the tau neutrino is a combination of the total kink and trident interaction efficiencies,

$$
\epsilon_{\nu_{\tau}}=B R(\tau \rightarrow \text { kink }) \times \epsilon_{\text {kink }}+B R(\tau \rightarrow \text { trid }) \times \epsilon_{\text {trid }}=0.49,
$$

where the branching ratios, $B R$, were measured by others [25]. Table 6.3 summarizes all of the types of efficiencies for each of the four event types.

The location efficiency was calculated by dividing the number of located events by the total number of events. Out of the total number of events, 1026, 181 of these events 


\section{Efficiencies (\%)}

\begin{tabular}{|c|c|c|c|c|}
\hline Type & $\nu_{\tau}$ kink cc & $\nu_{\tau}$ trident cc & $\nu_{e} \mathrm{cc}$ & $\nu_{\mu} \mathrm{cc}$ \\
\hline Trigger & 97 & 97 & 98 & 94 \\
\hline Selection & 80 & 80 & 80 & 80 \\
\hline Identification & 60 & 76 & 72 & 73 \\
\hline Total & 46 & 59 & 57 & 56 \\
\hline
\end{tabular}

Table 6.3: Summary of all efficiencies for electron, muon, tau kink, and tau trident chargedcurrent interactions.

were rejected for being outside the fiducial volume, which left 845 total events. Using 539 located events out of 845 total events, the location efficiency was calculated to be $64 \%$. This location efficiency was common to all of types of neutrino interactions using this method of calculation. The most common reason events were not located was the error in the spectrometer vertex prediction. Emulsion problems, such as slipping and distortions, existed, but were usually corrected in the analysis.

\subsubsection{Charged-Current Neutrino Cross Section}

The relative cross section measurement requires the constant part of the electron neutrino and muon neutrino cross sections. The constant part of the cross section was measured for the muon neutrino, and, according to lepton universality, should be equal to the constant part of the electron neutrino cross section. The averaged measured values [25] for the cross section in muon neutrino and muon anti-neutrino charged-current interactions are

$$
\begin{gathered}
\frac{\sigma_{\nu_{\mu} N}}{E}=\sigma_{\nu_{\mu} N}^{\text {const }}=0.677 \pm 0.0014 \times 10^{-38} \mathrm{~cm}^{2} \mathrm{GeV}^{-1} \\
\frac{\sigma_{\bar{\nu}_{\mu} N}}{E}=\sigma_{\bar{\nu}_{\mu} N}^{\text {const }}=0.334 \pm 0.008 \times 10^{-38} \mathrm{~cm}^{2} \mathrm{GeV}^{-1}
\end{gathered}
$$

Since neutrino and anti-neutrino interactions are indistinguishable and equal in the data, the measured cross section is an average; the value for $\sigma_{\nu_{\mu} N}^{\text {const }}$ and $\sigma_{\nu_{e} N}^{\text {const }}$ used in Eq. 6.9 is

$$
\sigma_{\nu N}^{\text {const }}=0.505 \pm 0.016 \times 10^{-38} \mathrm{~cm}^{2} \mathrm{GeV}^{-1}
$$

\subsubsection{The Kinematic Factor}

$K_{F}(E)$ is the factor which accounts for the differences in the kinematics of the the tau neutrino, electron neutrino, and muon neutrino interactions; the differences stem from the 


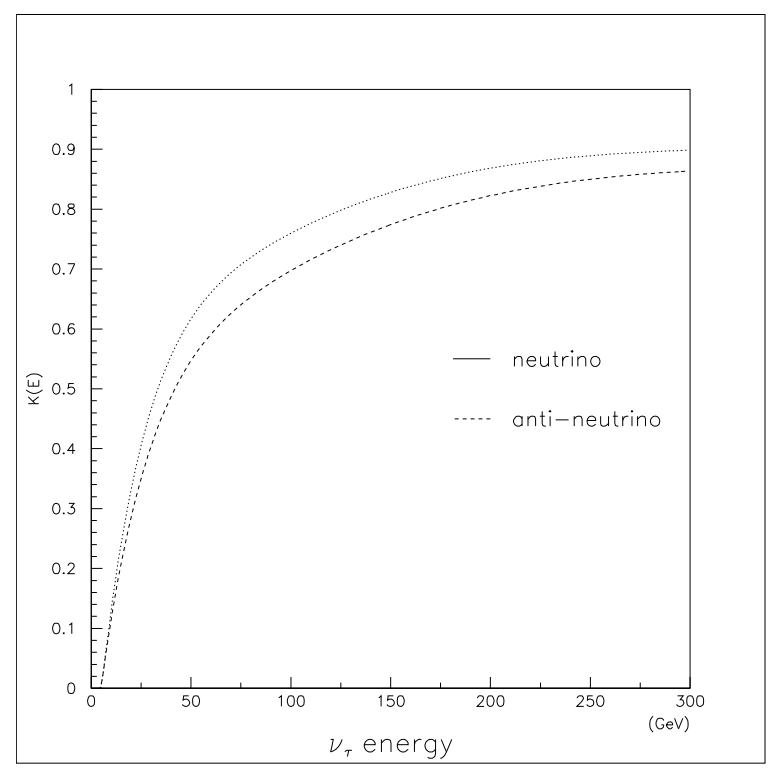

Figure 6.1: Kinematic factor for the tau neutrino (solid line) and the tau anti-neutrino (dashed line) [29].

masses of tau lepton, electron, and muon. This quantity was calculated numerically by Albright and Jarlskog [47]. The resulting distribution for $K_{F}(E)$ is shown in Figure 6.1.

\subsubsection{Neutrinos Traversing the Target}

The number of neutrinos which hit the target at a given energy was the product of the total number of neutrinos that were produced, $N_{\nu_{\tau}}^{\text {prod }}$, the target acceptance, $\eta$, and the energy spectrum, $\frac{d N_{\nu}^{\text {prod }}}{d E}$,

$$
N_{\nu_{\tau}}^{\operatorname{tar}}(E)=N_{\nu_{\tau}}^{\text {prod }} \times \eta \times \frac{d N_{\nu}^{\text {prod }}}{d E}
$$

In the relative cross section, the target acceptance canceled as it is the same for all types of neutrinos. $\frac{d N_{\nu}^{\text {prod }}}{d E}$ is the energy spectrum of the produced neutrinos, calculated using the Monte Carlo. The number of produced neutrinos, $N_{\nu_{\tau}}^{\text {prod }}$, is a function of the number of charm particles produced ${ }^{1}$. The number of charm particles produced is a function of the charm production cross sections for $800 \mathrm{GeV}$ protons on a tungsten target and charm

\footnotetext{
${ }^{1}$ This is only true for the prompt muon neutrinos. The non-prompt muon neutrinos are not used in this analysis
} 
semi-leptonic branching ratios. For the relative measurement, the number of neutrinos that traversed the target is

$$
\frac{N_{\nu_{\tau}}^{\operatorname{tar}}(E)}{N_{\nu_{e}}^{\operatorname{tar}}(E)}=\frac{\frac{N_{\nu \tau}^{\text {prod }}}{N_{\text {pot }}} \times \frac{d N_{\nu \tau}^{\text {prod }}}{d E}}{\frac{N_{\nu_{e}}^{\text {prod }}}{N_{\text {pot }}} \times \frac{d N_{\nu e}^{\text {prod }}}{d E}},
$$

where

$$
\frac{N_{\nu_{\alpha}}^{\text {prod }}}{N_{\text {pot }}}=\sum_{j} \frac{N_{\nu_{\alpha}}}{N_{c_{j}}} \times \frac{N_{c_{j}}}{N_{\text {pot }}},
$$

and $c_{j}$ are the relevant charm particles that produce the $\alpha$-type neutrinos, $\frac{N_{\nu_{\alpha}}}{N_{c_{j}}}$ is the number of $\alpha$-type neutrinos produced per charm particle, and $\frac{N_{c_{j}}}{N_{\mathrm{pot}}}$ is the number of charm particles per proton on the target. The number of neutrinos per charm particle is

$$
\frac{N_{\nu_{\alpha}}}{N_{c_{j}}}=\sum_{j} B R\left(c_{j} \rightarrow \nu_{\alpha} X\right),
$$

and the number of charm particles per proton on target is

$$
\frac{N_{\nu_{c_{j}}}}{\text { pot }}=\sum_{j} \frac{\sigma\left(p W \rightarrow c_{j} X\right)}{\sigma(p W)_{\text {total }}}
$$

where $\sigma\left(p W \rightarrow c_{j} X\right)$ are the charm production cross sections for $800 \mathrm{GeV}$ protons on tungsten and $B R\left(c_{j} \rightarrow \nu_{\tau} X\right)$ are the branching ratios for the charm particles to $\nu_{\tau}+X$, $\nu_{e}+X$, and $\nu_{\mu}+X . c_{j}$ can be $D_{s}, D^{ \pm}, D^{0}$, and $\Lambda_{c}$. Also included are the excited states, $D^{* \pm}$ and $D^{* 0}$ which decay to other charm particles, as discussed in Sec. 5.3.1.

The charm production cross sections were taken from several experiments, E691 [53], E769 [49], E653 [33], E743 [34], and NA32 [54]. A detailed discussion of the resulting charm production cross sections is included in [30]; the results are presented in Table 6.5.

The nuclear dependence of the production cross section of tungsten is written in terms of the production cross section of a nucleon with a scaling factor, $\alpha$,

$$
\sigma\left(p W \rightarrow c_{j} X\right)=A^{\alpha} \sigma\left(p N \rightarrow c_{j} X\right)
$$

where $A$ is the atomic number of tungsten $(W)$, the beam dump material, and $\sigma(p N \rightarrow$ $\left.c_{j} X\right)$ is the charm production cross section for a nucleon, $N$. The $\alpha$ dependence of the charm production cross section was measured by many experiments, and the values are shown in Table 6.4. Similarly, the total production cross section is scaled using

$$
\sigma(p W)_{\text {total }}=A^{\alpha} \sigma(p N)_{\text {total }}
$$




\section{Experimental $\alpha$ Values}

\begin{tabular}{|c|c|c|c|}
\hline Experiment & Beam type & Beam Energy $(\mathrm{GeV})$ & $\alpha$ \\
\hline WA82 [48] & $\pi$ & 340 & $0.92 \pm 0.06$ \\
\hline E769 [49] & $\pi$ & 250 & $1.0 \pm 0.05$ \\
\hline E789 [50] & $\mathrm{p}$ & 800 & $1.02 \pm 0.04$ \\
\hline WA92 [51] & $\pi$ & 350 & $0.95 \pm 0.03$ \\
\hline Average & & & $0.987 \pm 0.26$ \\
\hline
\end{tabular}

Table 6.4: Summary of $\alpha$ for the charm production cross section

where $\alpha$ for the total inelastic cross section for proton-nucleon collisions is 0.69 [52].

The ratio of the number of produced neutrinos is

$$
\begin{aligned}
\frac{N_{\nu_{\tau}}^{\text {prod }}}{N_{\nu_{e}}^{\text {prod }}}=\frac{2 \times A \times \sum_{j} \sigma\left(p N \rightarrow C_{j} X\right) B R\left(C_{j} \rightarrow \nu_{\tau} X\right)}{A^{\alpha} \times \sigma_{\text {tot }}(p N)} & \\
& \times \frac{A^{\alpha} \times \sigma_{\text {tot }}(p N)}{A \times \sum_{i} \sigma\left(p N \rightarrow C_{i} X\right) B R\left(C_{i} \rightarrow \nu_{\tau} X\right)} .
\end{aligned}
$$

The two in the numerator is necessary because each time a charm particle produced a tau neutrino, it also produced a tau lepton. The tau lepton subsequently decayed and produced another tau neutrino, the anti-particle of the first neutrino. Therefore, for every charm decay, a tau neutrino and a tau anti-neutrino were produced. The total proton production cross section and the atomic number cancel in the relative measurement. The branching ratios were taken from the particle data group, and are summarized in Table 6.6. The relative numbers of produced neutrinos are thus

$$
\frac{N_{\nu_{\tau}}^{\text {prod }}}{N_{\nu_{e}}^{\text {prod }}}=\frac{2 \times \sum_{j} \sigma\left(p N \rightarrow C_{j} X\right) B R\left(C_{j} \rightarrow \nu_{\tau} X\right)}{\sum_{i} \sigma\left(p N \rightarrow C_{i} X\right) B R\left(C_{i} \rightarrow \nu_{e} X\right)}=0.15 \pm 0.03,
$$

and similarly

$$
\frac{N_{\nu_{\tau}}^{\text {prod }}}{N_{\nu_{\mu}}^{\text {prod }}}=0.16 \pm 0.03
$$

In the relative measurement, the total production cross section of protons on a tungsten target canceled, but was necessary for the absolute cross section. The proton-nucleon total cross section for $800 \mathrm{GeV}$ protons has been measured to be $37.6 \mathrm{mb}$ [29]; thus

$$
\sigma(p W)_{\text {total }}=181^{0.69} \times 37.6 \mathrm{mb}=1358 \mathrm{mb}=1.36 \mathrm{~b} .
$$


Charm Production Cross Sections

\begin{tabular}{|l|r|}
\hline$\sigma\left(p N \rightarrow D_{s} X\right)$ & $5.2 \pm 0.8 \mu$ barn \\
\hline$\sigma\left(p N \rightarrow D^{ \pm} X\right)$ & $11.3 \pm 2.2 \mu$ barn \\
\hline$\sigma\left(p N \rightarrow D^{0} X\right)$ & $27.4 \pm 2.6 \mu$ barn \\
\hline$\sigma\left(p N \rightarrow \Lambda_{c} X\right)$ & $5.4 \pm 2.1 \mu$ barn \\
\hline
\end{tabular}

Table 6.5: Charm production cross sections for $800 \mathrm{GeV}$ protons.

\section{Branching Ratios}

\begin{tabular}{|l|r|}
\hline$B R\left(D_{s} \rightarrow \nu_{e} X\right)$ & $8 \pm 5.5 \%$ \\
\hline$B R\left(D_{s} \rightarrow \nu_{\tau} X\right)$ & $6.4 \pm 1.5 \%$ \\
\hline$B R\left(D_{s} \rightarrow \nu_{\mu} X\right)$ & $8 \pm 5.5 \%$ \\
\hline$B R\left(D^{ \pm} \rightarrow \nu_{e} X\right)$ & $17.2 \pm 1.9 \%$ \\
\hline$B R\left(D^{ \pm} \rightarrow \nu_{\tau} X\right)$ & $7 \times 10^{-4}$ \\
\hline$B R\left(D^{ \pm} \rightarrow \nu_{\mu} X\right)$ & $16.0 \pm 3.0$ \\
\hline$B R\left(D^{0} \rightarrow \nu_{e} X\right)$ & $6.9 \pm 0.3 \%$ \\
\hline$B R\left(D^{0} \rightarrow \nu_{\mu} X\right)$ & $6.6 \pm 0.8 \%$ \\
\hline$B R\left(\Lambda_{c} \rightarrow \nu_{e} X\right)$ & $2.1 \pm 0.7 \%$ \\
\hline$B R\left(\Lambda_{c} \rightarrow \nu_{\mu} X\right)$ & $2.0 \pm 0.6 \%$ \\
\hline$B R\left(\tau \rightarrow \nu_{e} X\right)$ & $17.84 \pm 0.06 \%$ \\
\hline$B R\left(\tau \rightarrow \nu_{\mu} X\right)$ & $17.36 \pm 0.06 \%$ \\
\hline
\end{tabular}

Table 6.6: Charm and tau lepton branching ratios. 
Then

$$
\begin{aligned}
\frac{N_{\nu_{\tau}}^{\text {prod }}}{N_{\text {pot }}} & =\sum_{j} B R\left(c_{j} \rightarrow \nu_{\tau} X\right) \times \frac{A \times \sigma\left(p N \rightarrow c_{j} X\right)}{A^{\alpha} \times \sigma(p W)_{\text {total }}} \\
= & \frac{181 \times 0.682 \mu \mathrm{b}}{1.36 \mathrm{~b}}=9.08 \pm 2.1 \times 10^{-5} \nu_{\tau} / \text { pot }
\end{aligned}
$$

and

$$
\begin{aligned}
\frac{N_{\nu_{e}}^{\text {prod }}}{N_{\text {pot }}} & =\sum_{j} B R\left(c_{j} \rightarrow \nu_{e} X\right) \times \frac{A \times \sigma\left(p N \rightarrow c_{j} X\right)}{\left.A^{\alpha} \times \sigma(p W)\right)_{\text {total }}} \\
= & \frac{181 \times 4.41 \mu \mathrm{b}}{1.36 \mathrm{~b}}=5.88 \pm 1.4 \times 10^{-4} \nu_{e} / \text { pot }
\end{aligned}
$$

\subsubsection{Produced Energy Spectra}

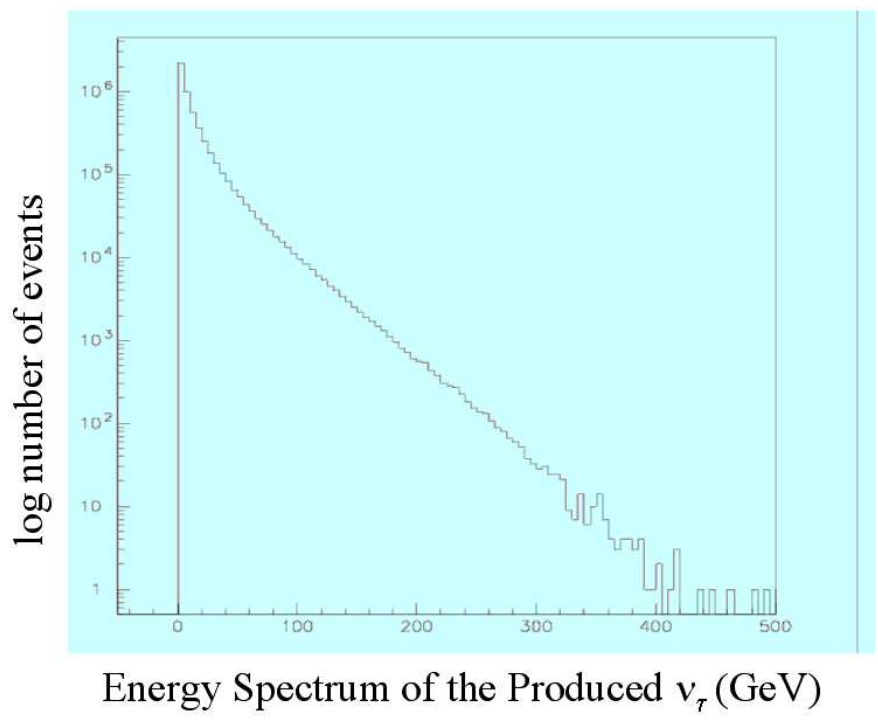

Figure 6.2: The energy spectrum of produced tau neutrinos.

The energy spectra, $\frac{d N_{\nu}^{\text {prod }}}{d E}$, for the produced electron, muon, and tau neutrinos were generated using the Monte Carlo, as described in Sec. 4.4. Fig. 6.2 shows the energy spectrum of the produced tau neutrinos. Fig. 6.3 shows the energy spectrum of the produced electron neutrinos. 


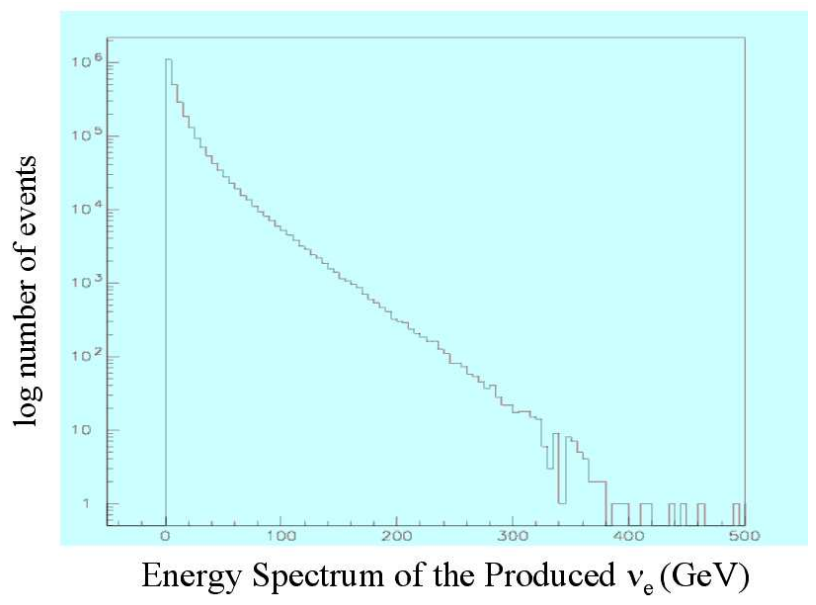

Figure 6.3: The energy spectrum of produced electron neutrinos.

The energy dependent part of Eq. 6.9 is

$$
\frac{\int E_{\nu_{\tau}} \times K_{F}(E) \times \frac{d N_{\nu_{\tau}}^{\text {prod }}}{d E} d E}{\int E_{\nu_{e}} \times \frac{d N_{\nu_{e}}^{\text {prod }}}{d E} d E}=0.320 \pm 0.002 .
$$

The efficiencies were removed from the integral because of their weak energy dependence, as discussed in Sec. 6.2.2. This integral was calculated using two different types of numerical integration: the Riemann method [55] and the Monte Carlo method [55]. The results from the two methods agreed to within $0.06 \%$. Similarly this fraction was calculated for the muon neutrinos; the value is

$$
\frac{\int E_{\nu_{\tau}} \times K_{F}(E) \times \frac{d N_{\nu_{\tau}}^{\text {prod }}}{d E} d E}{\int E_{\nu_{\mu}} \times \frac{d N_{\nu_{\mu}}^{\text {prod }}}{d E} d E}=0.348 \pm 0.002 .
$$

\subsubsection{The Number of Scattering Centers}

The number of scattering centers per proton, $N_{\text {scat }}$, was a characteristic of the target; therefore, it was the same for each type of neutrino interaction, but was required for the absolute cross section measurement. Since the neutrinos interacted with nucleons, the number of scattering centers was the number of nucleons in the target,

$$
N_{\text {scat }}=\frac{M_{\mathrm{tar}}}{A \times M}
$$




\section{Scattering Centers}

\begin{tabular}{|c|c|c|c|}
\hline Module & Composition & $N_{\text {scat }} / \mathrm{cm}^{2}$ & Periods \\
\hline 1 & ECC2 & $2.40 \times 10^{25}$ & $1,2,3$ \\
\hline 2 & ECC3 & $2.40 \times 10^{25}$ & 1,2 \\
\hline 3 & E/B1 & $1.64 \times 10^{25}$ & 4 \\
\hline 4 & E/B2 & $1.59 \times 10^{25}$ & 3,4 \\
\hline 5 & E/B3 & $1.57 \times 10^{25}$ & 3,4 \\
\hline 6 & E/B 4 & $1.59 \times 10^{25}$ & 2,3 \\
\hline 7 & $\mathrm{~B} 4$ & $1.59 \times 10^{25}$ & 4 \\
\hline
\end{tabular}

Table 6.7: The number of scattering centers per $\mathrm{cm}^{2}$ in each module for each period.

where $M_{\mathrm{tar}}$ is the mass of the target, $M$ is the mass of a nucleon, and $A$ is the cross sectional area of the target. $M_{\mathrm{tar}}$ is different for each module because the modules were each made of a distinct configuration of emulsion sheets and were exposed for different lengths of time. The average mass of the proton and neutron, $1.674 \times 10^{-27} \mathrm{~kg}$, was used in this calculation since an equal number of protons and neutrons existed in the target. The area was the same for each module,

$$
A=50 \mathrm{~cm} \times 50 \mathrm{~cm}=2500 \mathrm{~cm}^{2} .
$$

Using these values, Eq. 6.29, and the mass of each module from Table 3.1, the number of scattering centers per area was calculated for each module. The results are summarized in Table 6.7.

\subsubsection{The Number of Protons on Target}

The numbers of protons on the target were measured separately during each of the four periods of data-taking and are summarized in the Table 6.8. The uncertainty in these values was $15 \%$ [30] and is discussed in Sec. 6.3.2. Using the information in Table 6.7 and 6.8

$$
N_{\text {scat }} \times N_{\text {pot }}=2.27 \times 10^{43} \operatorname{pot} / \mathrm{cm}^{2} .
$$

\subsubsection{Acceptance}

Using the Monte Carlo the tau neutrinos from both the decay of the $D_{s}$ particle and the tau lepton decay were projected to the emulsion target, $36 \mathrm{~m}$ from the tungsten beam dump. 


Protons on Target
\begin{tabular}{|c|c|}
\hline Period & pot \\
\hline 1 & $5.4 \times 10^{16}$ \\
\hline 2 & $4.4 \times 10^{16}$ \\
\hline 3 & $1.03 \times 10^{17}$ \\
\hline 4 & $1.55 \times 10^{17}$ \\
\hline
\end{tabular}

Table 6.8: The number of protons on target for each period.

The target acceptance was the fraction which lay within the $50 \mathrm{~cm} \times 50 \mathrm{~cm}$ transverse dimensions of the target, which was

$$
\eta=0.064[41]
$$

This does not include corrections for the fiducial volume, which were accounted for in the efficiencies. Using the acceptance and number of produced neutrinos calculated in the previous section, the numbers of tau and electron neutrinos which traveled through the target are

$$
N_{\nu_{\tau}}^{\mathrm{tar}}(E)=9.08 \pm 2.1 \times 10^{-5} \nu_{\tau} / \operatorname{pot} \times 0.064 \times \frac{\mathrm{dN}_{\nu_{\tau}}^{\text {prod }}}{\mathrm{dE}}
$$

and

$$
N_{\nu_{e}}^{\operatorname{tar}}(E)=5.88 \pm 1.3 \times 10^{-4} \nu_{\tau} / \text { pot } \times 0.064 \times \frac{\mathrm{dN}_{\nu_{\mathrm{e}}}^{\mathrm{prod}}}{\mathrm{dE}}
$$

\subsection{Uncertainties}

\subsubsection{The Statistical Uncertainties}

Two main sources caused the statistical uncertainties in this measurement. The first, and largest, source was a statistical error which stemmed from the number of detected events. This limitation accounted for a $45 \%$ error on this measurement, as it is proportional to $\sqrt{N} / N[56]$. The second source of error came from the uncertainty in the charm branching ratios and charm production cross sections for $800 \mathrm{GeV}$ protons.

\subsubsection{The Systematic Uncertainties}

The largest systematic uncertainties stemmed from the number of protons on target; which was estimated to be 15\% [30]. The parameters, $b$ and $n$ were used in the Monte Carlo, as 
described in Sec. 4.4, and determined the energy and angle distributions; these parameter were known within $8 \%$ [28]. Although no systematic differences between the Monte Carlo and data were observed, an additional $5 \%$ systematic uncertatity was assigned to reflect the belief in the accuracy of the Monte Carlo [30]. These systematic errors only affected the absolute measurements, as they canceled in the relative measurements.

\subsection{Results}

This section presents three measurements of the tau neutrino, two measurements of the electron neutrino, and a measurement of the muon neutrino charged-current cross sections in the deep inelastic scattering region. The first measured the tau neutrino cross section relative to the electron neutrino cross section. The second measured the tau neutrino cross section relative to the muon neutrino cross section. The third measured the electron neutrino cross section relative to the muon neutrino cross section. The fourth measured the absolute cross section of the tau neutrino. The fifth measured the absolute cross section of the electron neutrino. The sixth measures the absolute cross section of the muon neutrino. These measurements are compared to their predicted or previously measured values in this section.

\subsubsection{Relative Measurement of Tau to Electron}

The charged-current interaction cross section for the tau neutrino relative to the electron neutrino cross section is

$$
\frac{\sigma_{\nu_{\tau} N}^{\text {const }}}{\sigma_{\nu_{e} N}^{\text {const }}}=\frac{N_{\nu_{\tau}}^{\text {obs }}}{N_{\nu_{e}}^{\text {obs }}} \times \frac{\epsilon_{\nu_{e}}}{\epsilon_{\nu_{\tau}}} \times \frac{N_{\nu_{e}}^{\text {prod }}}{N_{\nu_{\tau}}^{\text {prod }}} \times \frac{\int E_{\nu_{e}} \times \frac{d N_{\nu_{e}}^{\text {prod }}}{d E} \times d E}{\int K_{F}(E) \times E_{\nu_{\tau}} \times \frac{d N_{\nu_{\tau}}^{\text {prod }}}{d E} \times d E} .
$$

Using the quantities calculated in the previous section, the relative cross section is

$$
\begin{gathered}
\frac{\sigma_{\nu_{\tau} N}^{\text {const }}}{\sigma_{\nu_{e} N}^{\text {const }}}=\frac{5 \pm \sqrt{5}}{153 \pm \sqrt{153}} \times \frac{0.57}{0.49} \times 6.5 \pm 1.5 \times 3.1 \pm 0.02 \\
\frac{\sigma_{\nu_{\tau} N}^{\text {const }}}{\sigma_{\nu_{e} N}^{\text {const }}}=0.77 \pm 0.35 \pm 0.18
\end{gathered}
$$

where the two main sources of error have been separated. The first error quoted is from the statistical error due to the limited number of event. The second error quoted is from the uncertainty in the charm branching ratios and production cross section uncertainties. 


\subsubsection{Relative Measurement of Tau to Muon}

The charged-current interaction tau neutrino cross section relative to the muon neutrino cross section is

$$
\frac{\sigma_{\nu_{\tau} N}^{\text {const }}}{\sigma_{\nu_{\mu} N}^{\text {const }}}=\frac{N_{\nu_{\tau}}^{\text {obs }}}{N_{\nu_{\mu}}^{\text {obs }}} \times \frac{\epsilon_{\nu_{\mu}}}{\epsilon_{\nu_{\tau}}} \times \frac{N_{\nu_{\mu}}^{\text {prod }}}{N_{\nu_{\tau}}^{\text {prod }}} \times \frac{\int E_{\nu_{\mu}} \times \frac{d N_{\nu_{\mu}}^{\text {prod }}}{d E} \times d E}{\int K_{F}(E) \times E_{\nu_{\tau}} \times \frac{d N_{\nu_{\tau}}^{\text {prod }}}{d E} \times d E},
$$

where all muon neutrino terms were the values for prompt ${ }^{2}$ muon neutrinos. Using the quantities calculated in the previous section, the relative cross section using the prompt muon neutrino charged-current interactions is

$$
\frac{\sigma_{\nu_{\tau} N}^{\text {const }}}{\sigma_{\nu_{\mu} N}^{\text {const }}}=\frac{5 \pm \sqrt{5}}{214 \pm \sqrt{214} \times f_{p}} \times \frac{0.56}{0.49} \times 6.1 \pm 1.1 \times 2.9 \pm 0.02,
$$

where $f_{p}$ is the fraction of prompt muon neutrinos and anti-neutrinos. The determination of $f_{p}$ is the subject of [28]. In this reference, the muon energy spectrum was simulated for both the prompt and non-prompt muon neutrino interactions. The DONUT muon spectrum was then fit to the simulated spectra to measure the fraction of the prompt muon neutrino interactions in the data. The result was $f_{p}=0.60_{-0.10}^{+0.11}$. Using this value of $f_{p}$, the relative cross section is

$$
\frac{\sigma_{\nu_{\tau} N}^{\text {const }}}{\sigma_{\nu_{\mu} N}^{\text {const }}}=0.78 \pm 0.38 \pm 0.14
$$

The first error quoted includes the statistical error and the error on $f_{p}$.

\subsubsection{Relative Measurement of Electron to Muon}

As a check of the technique, the charged-current interaction cross section for the electron neutrino was calculated relative to the muon neutrino cross section using

$$
\frac{\sigma_{\nu_{e} N}^{\text {const }}}{\sigma_{\nu_{\mu} N}^{\text {const }}}=\frac{N_{\nu_{e}}^{\text {obs }}}{N_{\nu_{\mu}}^{\text {obs }}} \times \frac{\epsilon_{\nu_{\mu}}}{\epsilon_{\nu_{e}}} \times \frac{N_{\nu_{\mu}}^{\text {prod }}}{N_{\nu_{e}}^{\text {prod }}} \times \frac{\int E_{\nu_{\mu}} \times \frac{d N_{\nu_{\mu}}^{\text {prod }}}{d E} \times d E}{\int E_{\nu_{e}} \times \frac{d N_{\nu_{e}}^{\text {prod }}}{d E} \times d E}
$$

where all muon neutrino terms are the values for prompt muon neutrinos. Using the quantities calculated in the previous section, the electron neutrino cross section relative

\footnotetext{
${ }^{2}$ Prompt neutrinos were created in charm decays, while non-prompt neutrinos were created in the decay of light mesons.
} 
to the muon neutrino cross section is

$$
\frac{\sigma_{\nu_{e} N}^{\text {const }}}{\sigma_{\nu_{\mu} N}^{\text {const }}}=1.0 \pm 0.20 \pm 0.13
$$

According to lepton universality, this ratio should be 1.0. The result is consistent with 1.0 .

\subsubsection{Absolute Measurement of the Cross Sections}

This section presents the absolute cross section measurements for tau neutrinos, electron neutrinos, and muon neutrinos. Beginning with Eq. 6.43,

$$
N_{\nu_{\alpha}}^{\text {obs }}=N_{\text {scat }} \times N_{\text {pot }} \times \epsilon_{\nu_{\alpha}} \times \int N_{\nu_{\alpha}}^{\mathrm{tar}}(E) \times \sigma_{\nu_{\alpha} N}(E) d E
$$

The absolute measurement of the tau neutrino cross section is

$$
\sigma_{\nu_{\tau} N}^{\text {const }}=\frac{\sigma_{\nu_{\tau} N}}{E}=\frac{N_{\nu_{\tau}}^{\text {obs }}}{N_{\text {scat }} \times N_{\text {pot }} \times \epsilon_{\nu_{\tau}} \times \int N_{\nu_{\tau}}^{\text {tar }}(E) \times K_{F}(E) \times E_{\nu_{\tau}} d E} .
$$

Using Eq. 6.44 and the quantities discussed above, the absolute charged current cross section of the tau neutrino is

$$
\sigma_{\nu_{\tau} N}^{\text {const }}=0.43 \pm 0.18 \pm 0.10 \pm 07 \text { (sys) } \times 10^{-38} \mathrm{~cm}^{2} \mathrm{GeV}^{-1}
$$

where the first error is from the limited number of events, the second error is from the uncertainties in the charm parameters, and the last error is due to systematic errors. The absolute charged-current cross section of the electron neutrino is

$$
\sigma_{\nu_{e} N}^{\text {const }}=0.53 \pm 0.08 \pm 0.12 \pm 0.10 \text { (sys) } \times 10^{-38} \mathrm{~cm}^{2} \mathrm{GeV}^{-1} \text {. }
$$

The absolute measurement of the muon neutrino cross section is

$$
\sigma_{\nu_{\mu} N}^{\text {const }}=0.47 \pm 0.09 \pm 0.12 \pm 0.08 \text { (sys) } \times 10^{-38} \mathrm{~cm}^{2} \mathrm{GeV}^{-1}
$$

The last two measurements are known experimentally [25],

$$
\sigma_{\nu_{\mu} N}^{\text {const }}=\sigma_{\nu_{e} N}^{\text {const }}=0.505 \pm 0.012 \times 10^{-38} \mathrm{~cm}^{2} \mathrm{GeV}^{-1} \text {. }
$$

These measurements do not show a strong systematic bias in the technique. Both of these measurements are well within the estimated uncertainty. 


\subsubsection{Consistency with Theoretical Data}

If the tau neutrino is a standard model particle, the cross section should be identical to the muon neutrino and anti-neutrino,

$$
\frac{\sigma_{\nu_{\tau} N}^{\text {const }}}{\sigma_{\nu_{\mu} N}^{\text {const }}}=1.0
$$

The measurements of the ratio using the DONUT data are

$$
\frac{\sigma_{\nu_{\tau} N}^{\text {const }}}{\sigma_{\nu_{e} N}^{\text {const }}}=0.77 \pm 0.35 \pm 0.18
$$

using the electron neutrinos, and

$$
\frac{\sigma_{\nu_{\tau} N}^{\text {const }}}{\sigma_{\nu_{\mu} N}^{\text {const }}}=0.78 \pm 0.38 \pm 0.14 .
$$

using the muon neutrinos. Fig. 6.4 shows the measurements of the ratios of tau neutrino to the electron neutrino, the tau neutrino to the muon neutrino, the electron neutrino to the muon neutrino, and the predicted value. The errors bars on these measurements only take into account the largest error, the error due to the limited number of events. The results are consistent with the predicted values. 


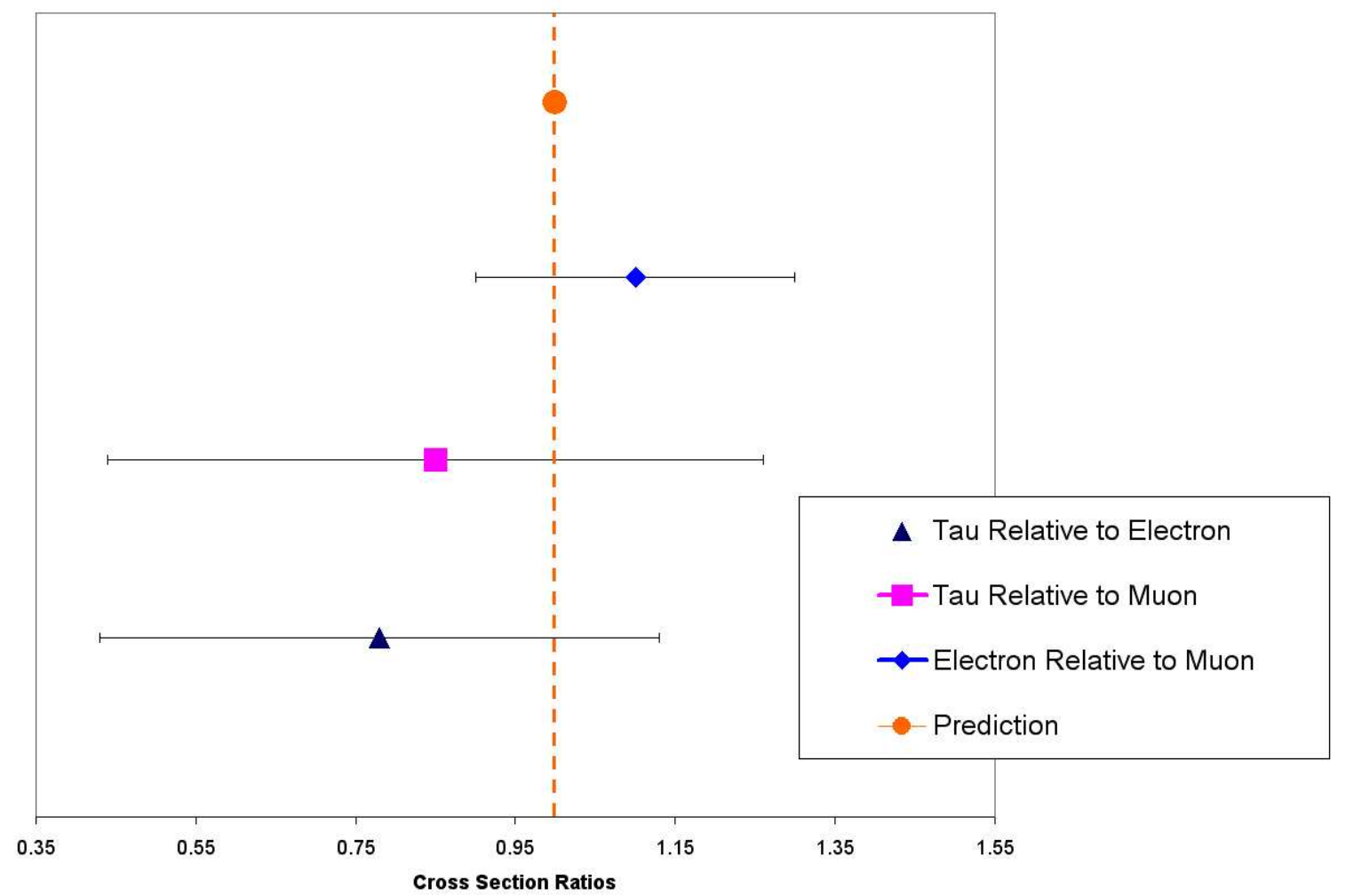

Figure 6.4: The cross section measurements of the ratios of tau neutrino to the electron neutrino, the tau neutrino to the muon neutrino, the electron neutrino to the muon neutrino, and the predicted value. The errors bars on these measurements only take into account the largest error, the error due to the limited number of events. 
From Eq. 2.23, the prediction for and equal number of $115 \mathrm{GeV}$ neutrinos and antineutrinos is

$$
\sigma_{\nu_{\tau} N}^{\mathrm{SM}}=48 \pm 5 \times 10^{-38} \mathrm{~cm}^{2}
$$

To compare this theoretical values to the measured tau to electron ratio,

$$
\begin{aligned}
\sigma_{\nu_{\tau} N}^{\exp } & =\frac{\sigma_{\nu_{\tau} N}^{\text {const }}}{\sigma_{\nu_{e} N}^{\text {const }}} \times \sigma_{\nu_{e} N}^{\text {const }} \times E_{\nu_{\tau}} \\
& =45 \pm 21 \pm 10 \times 10^{-38} \mathrm{~cm}^{2}
\end{aligned}
$$

where $\sigma_{\nu_{e} N}^{\text {const }}=0.505 \pm 0.012 \times 10^{-38} \mathrm{~cm}^{2} \mathrm{GeV}^{-1}$ and $E_{\nu_{\tau}}=115 \mathrm{GeV}$. Similarly for the tau to muon measured ratio,

$$
\begin{aligned}
\sigma_{\nu_{\tau} N}^{\text {exp }} & =\frac{\sigma_{\nu_{\tau} N}^{\text {const }}}{\sigma_{\nu_{\mu} N}^{\text {const }}} \times \sigma_{\nu_{\mu} N}^{\text {const }} \times E_{\nu_{\tau}} \\
& =45 \pm 22 \pm 10 \times 10^{-38} \mathrm{~cm}^{2}
\end{aligned}
$$

To compare the absolute value to the theoretical prediction,

$$
\begin{aligned}
\sigma_{\nu_{\tau}}^{\exp } & =\sigma_{\nu_{\tau}}^{\text {const }} \times E_{\nu_{\tau}} \\
& =50 \pm 22 \pm 10 \pm 8(\text { sys }) \times 10^{-38} \mathrm{~cm}^{2} .
\end{aligned}
$$

where $E_{\nu_{\tau}}=115 \mathrm{GeV}$. Fig. 6.5 shows all the cross section measurements evaluated at $115 \mathrm{GeV}$ : the predicted value, the absolute measurement, the measurement relative to the electron neutrinos, and the measurement relative to the muon neutrinos. The errors bars represent the largest error, the statistical error due to the limited number of events. All values are consistent within error; therefore the results of this analysis support the standard model prediction for the charged-current tau neutrino interaction cross section in the deep inelastic scattering region. 


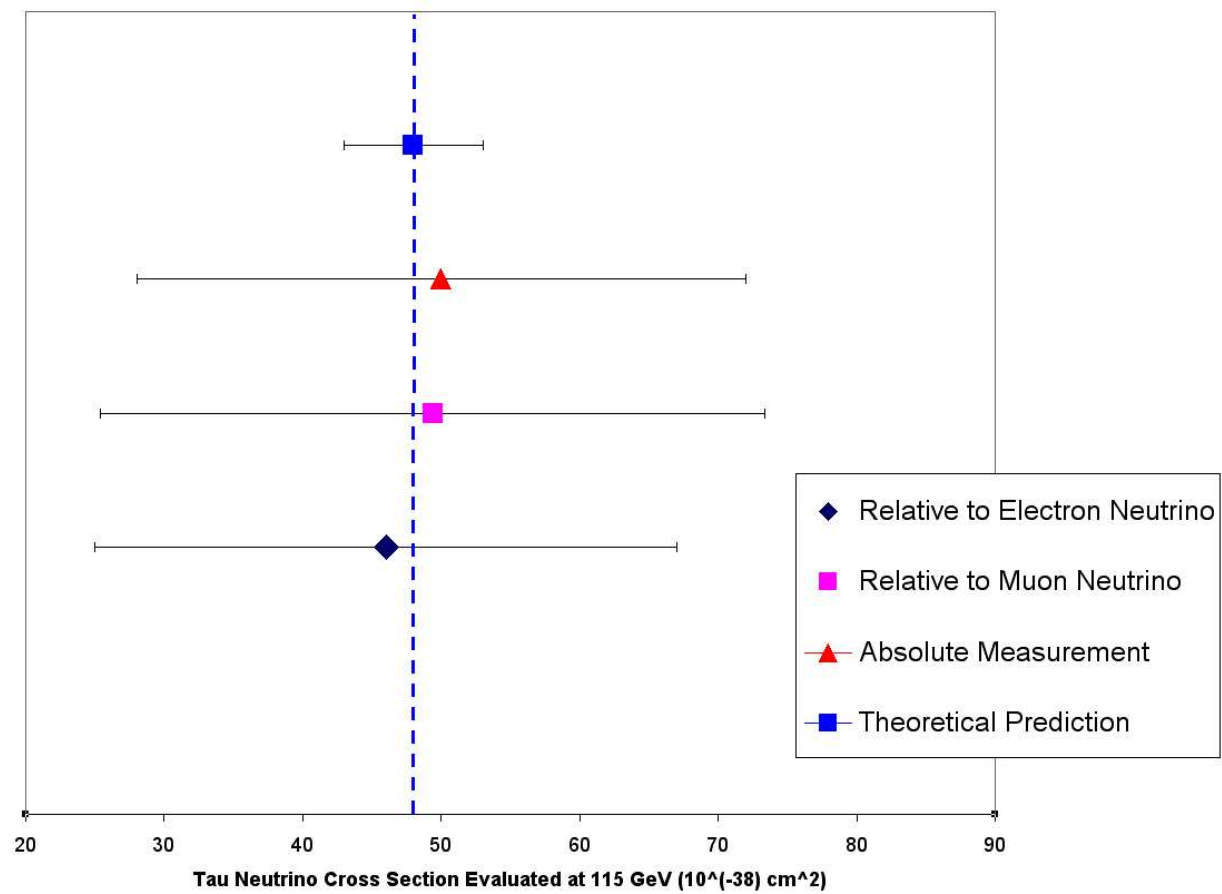

Figure 6.5: The tau neutrino cross sections evaluated at $115 \mathrm{GeV}$, the average energy of the interacted tau neutrinos. The predicted value, the absolute measurement, the measurement relative to the electron neutrinos, and the measurement relative to the muon neutrinos are all shown. The errors bars represent the largest error, the statistical error due to the limited number of events. 


\section{Chapter 7}

\section{Conclusion}

The charged-current interaction cross section of the tau neutrino in the deep inelastic scattering region was measured using three methods in this thesis. The primary measurement used the electron neutrino interactions to make a relative measurement of the tau neutrino cross section. Similarly, the secondary measurement used muon neutrino interactions. The tertiary measurement was an absolute measurement of the tau neutrino cross section. The techniques used to make these measurements were also used to measure the absolute electron and muon cross sections and measure the electron neutrino cross section relative to the muon neutrino cross section. Since the electron and muon neutrino cross sections are well known, the measured and actual values were compared to test the validity of the technique.

Two main sources caused the statistical uncertainties in these measurements. The first, and largest, source was a statistical error which stemmed from the limited number of events used in the calculation. This limitation accounted for a $45 \%$ error on this measurement, as it is proportional to $\sqrt{N} / N$. The second source of error came from the uncertainty in the charm branching ratios and charm production cross sections for 800 $\mathrm{GeV}$ protons. As the charm branching ratios and production cross sections are measured more accurately, the error on this measurement will decrease; however, the uncertainty from the limited number of events is dominant. In order to increase the accuracy of this measurement, more tau neutrino interactions are necessary. This is achieved with a more intense beam of neutrinos, higher energy neutrinos, or a longer exposure time. Since there are no plans to perform another fixed target experiment to study tau neutrino interactions, this measurement will not improve much in the near future. 
Systematic uncertainties stemmed from the number of protons on target, the parameters, $b$ and $n$, which were used in the Monte Carlo, and an additional $5 \%$ systematic uncertatity assigned to reflect the belief in the accuracy of the Monte Carlo. These systematic errors only affected the absolute measurements, as they canceled in the relative measurements.

The primary measurement, which measured the tau neutrino charged-current cross section relative to the electron neutrino charged-current cross section in the deep inelastic scattering range, is

$$
\frac{\sigma_{\nu_{\tau} N}^{\text {const }}}{\sigma_{\nu_{e} N}^{\text {const }}}=0.77 \pm 0.35 \pm 0.18
$$

where the two main sources of error have been separated. The first error quoted is the statistical error due to the limited number of event. The second error quoted is from the uncertainties in the charm branching ratios and production cross sections which were used in the calculation of the number of neutrinos produced.

The secondary measurement used the muon neutrino interactions instead of the electron neutrino interactions. The ratio of prompt $^{1}$ muon neutrino interactions to nonprompt was required for this calculation. The resulting ratio is

$$
\frac{\sigma_{\nu_{\tau} N}^{\text {const }}}{\sigma_{\nu_{\mu} N}^{\text {const }}}=0.78 \pm 0.38 \pm 0.14
$$

The absolute measurements of the tau neutrino cross section is

$$
\sigma_{\nu_{\tau}}^{\text {const }}=0.43 \pm 0.18 \pm 0.10 \pm 0.07 \text { (sys) } \times 10^{-38} \mathrm{~cm}^{2} \mathrm{GeV}^{-1} \text {. }
$$

Lepton universality predicts these ratios should be 1.0. All three measurements are consistent with this prediction.

To compare these values to the prediction derived in Ch. 2, each cross section was evaluated at $115 \mathrm{GeV}$, which was the average energy of the interacted neutrinos. The predicted cross section for $115 \mathrm{GeV}$ neutrinos is

$$
\sigma_{\nu_{\tau} N}^{\mathrm{SM}}=48 \pm 5 \times 10^{-38} \mathrm{~cm}^{2} .
$$

The measurement relative to the electron neutrino cross section yields

$$
\sigma_{\nu_{\tau} N}^{\exp }=\frac{\sigma_{\nu_{\tau} N}^{\text {const }}}{\sigma_{\nu_{e} N}^{\text {const }}} \times \sigma_{\nu_{e} N}^{\text {const }} \times E_{\nu_{\tau}}=45 \pm 21 \pm 10 \times 10^{-38} \mathrm{~cm}^{2} .
$$

\footnotetext{
${ }^{1}$ Prompt muon neutrinos were produced from charm decays, while non-prompt muon neutrinos were produced in the decay of light mesons
} 
The measurement relative to the muon neutrino cross section yields

$$
\sigma_{\nu_{\tau} N}^{\exp }=\frac{\sigma_{\nu_{\tau} N}^{\text {const }}}{\sigma_{\nu_{\mu} N}^{\text {const }}} \times \sigma_{\nu_{\mu} N}^{\text {const }} \times E_{\nu_{\tau}}=45 \pm 22 \pm 10 \mathrm{~cm}^{2} .
$$

For the absolute value, the result was multiplied by the energy

$$
\sigma_{\nu_{\tau}}^{\exp }=\sigma_{\nu_{\tau}}^{\text {const }} \times E=50 \pm 22 \pm 10 \pm 8 \text { (sys) } \mathrm{cm}^{2} .
$$

Each of these measurement are consistent with the prediction; therefore, the results support the standard model.

To test the validity of this technique, the electron and muon neutrino cross sections were measured. If the techniques used in this analysis are sound, the measurements should yield the known values which have been found experimentally. The electron neutrino charged-current cross section was measured relative to muon neutrino cross section. In addition, the absolute electron and muon neutrino cross sections were measured.

$$
\frac{\sigma_{\nu_{e} N}^{\text {const }}}{\sigma_{\nu_{\mu} N}^{\text {const }}}=1.0 \pm 0.20 \pm 0.13
$$

Due to lepton universality this ratio should be equal to 1.0. The measurement yielded a result consistent with the expected value. The absolute cross section of the electron neutrino is

$$
\sigma_{\nu_{e}}^{\text {const }}=0.53 \pm 0.08 \pm 0.12 \pm 0.10(\text { sys }) \times 10^{-38} \mathrm{~cm}^{2} \mathrm{GeV}^{-1}
$$

and the absolute muon neutrino cross section is

$$
\sigma_{\nu_{\mu} N}^{\text {const }}=0.47 \pm 0.09 \pm 0.12 \pm 0.08(\text { sys }) \times 10^{-38} \mathrm{~cm}^{2} \mathrm{GeV}^{-1} .
$$

The electron and muon neutrino cross sections are known from past experiments,

$$
\sigma_{\nu_{\mu} N}^{\text {const }}=\sigma_{\nu_{e} N}^{\text {const }}=0.505 \pm 0.012 \times 10^{-38} \mathrm{~cm}^{2} \mathrm{GeV}^{-1} \text {. }
$$

The electron measurement is approximately $4 \%$ higher than the actual values, while the muon measurement is approximately $7 \%$ lower than the actual value. Both of these measurements are well within the estimated uncertainty.

This is the first measurement of the tau neutrino charged-current interaction cross section, and it is consistent with the standard model. In addition to testing the standard model, the cross section is important to neutrino oscillation experiments. The OPERA experiment, for example, is searching for the appearance of the tau leptons from tau neutrino 
charged-current interactions in a beam of muon neutrinos. Since the OPERA experiment will not have a near detector, it requires the tau neutrino cross section to determine oscillation parameters. The oscillation parameters are used to study the necessary extensions to the standard model.

The DONUT experiment observed tau neutrino charged-current interactions for the first time. These interactions were used to measure the charged-current interaction cross section for the tau neutrino in the deep inelastic scattering region. The results from this thesis show internal consistency and are consistent with the standard model. 


\section{Appendix A}

\section{The Deep Inelastic Scattering Region}

The tau neutrino cross section is well understood in two energy regions: the low energy region, in which the neutrino scatters elastically from the entire nucleon, and the high energy region, in which the neutrino scatters inelastically from the partons inside the nucleon. The neutrino energies in the DONUT experiment were typically in the high energy region, as the following calculations illustrate.

\section{A.1 $Q^{2}$ and $W^{2}$}

$Q^{2}$ and $W^{2}$ are invariants used to describe deep inelastic scattering as discussed in Ch. 2. $W^{2}$ is defined as

$$
W^{2}=(P+q)^{2}=P^{2}-Q^{2}+2 P \cdot q=M^{2}-Q^{2}+2 M\left(E_{\nu_{\tau}}-E_{\tau}\right),
$$

where $P$ is the momentum four-vector of the nucleon, $M$ is the mass of the nucleon, $E_{\nu_{\tau}}$ is the energy of the tau neutrino, $E_{\tau}$ is the energy of the tau lepton, and $Q^{2}$ is

$$
Q^{2}=-q^{2}=-\left(k-k^{\prime}\right)^{2}=-m_{\tau}^{2}+E_{\nu_{\tau}}\left(E_{\tau}-p_{\tau} \cos \theta\right)
$$

where $k$ is the momentum four-vector of the neutrino and $k^{\prime}$ is the momentum four-vector of the tau lepton, as shown in Fig. 2.3.

At high energies, neutrino scattering is called deep inelastic scattering. It is deep when $Q^{2}>>M^{2}$ and inelastic when $W^{2}>>M^{2} . Q^{2}$ and $W^{2}$ are calculated for each tau neutrino charged-current interaction candidate. 


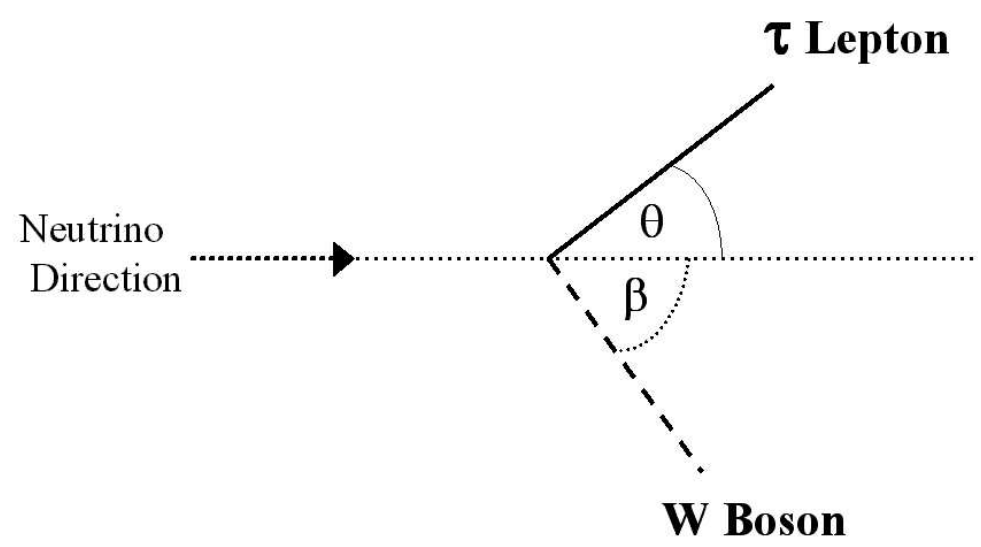

Figure A.1: The angles used in the calculation of the tau neutrino energy. $\beta$ is the angle between the $W^{ \pm}$boson and the neutrino, and $\theta$ is the angle between the tau lepton and the neutrino.

\section{A.2 Estimating the Energy of the Tau Neutrino}

The neutrino energy could not be measured. Momentum conservation provides an estimate for $E_{\nu_{\tau}}$. In the beam direction

$$
p_{\nu_{\tau}}=p_{\tau} \cos \theta+p_{W} \cos \beta
$$

where $\beta$ is the angle between the $W^{ \pm}$boson and the neutrino, and $\theta$ is the angle between the tau lepton and the neutrino, as shown in Fig. A.1.

The primary hadrons originated from the $W^{ \pm}$boson produced in the neutrino interactions; therefore, the net direction of the hadrons was the direction of the boson. $\beta$ is the angle between the neutrino direction and the direction of the vector sum of the charged primary hadrons. This was an estimate for the boson direction as the neutral hadrons are not included. Momentum is conserved in the transverse direction, which yields

$$
0=p_{\tau} \sin \theta-p_{W} \sin \beta
$$

Combining the equations and solving for $E_{\nu_{\tau}}$ yields

$$
p_{\nu_{\tau}}=p_{\tau} \cos \theta+\frac{p_{\tau} \sin \theta}{\sin \beta} \cos \beta=p_{\tau}\left(\cos \theta+\frac{\sin \theta}{\tan \beta}\right)=E_{\nu_{\tau}} .
$$

Since the mass of the neutrino is approximately zero, $E_{\nu_{\tau}}=p_{\nu_{\tau}}$. 


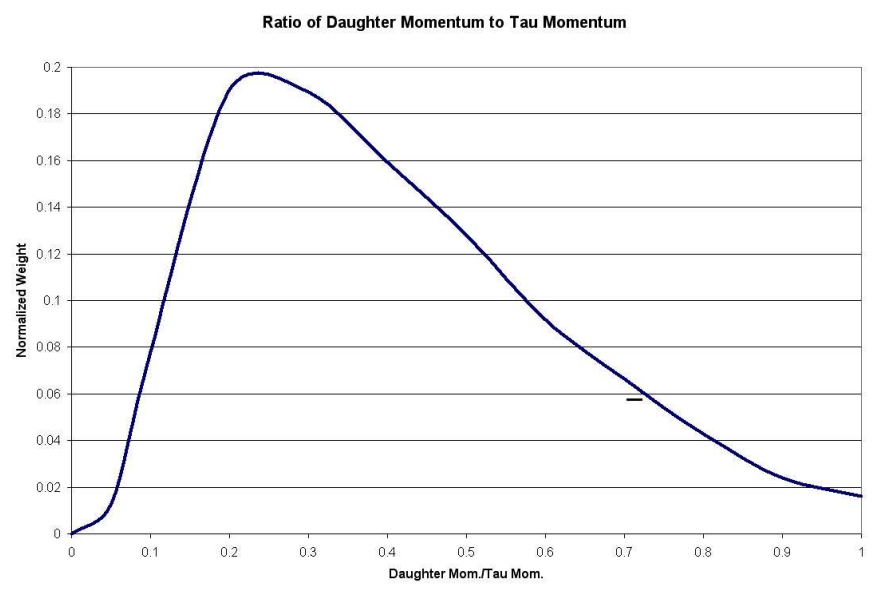

Figure A.2: A comparison of the daughter's momentum and the tau lepton's momentum using Monte Carlo data for the kink events. The X-axis is the daughter's momentum divided by the tau lepton's momentum. The Y-axis is the normalized weight of the events. If the daughter's momentum and the tau lepton's momentum were equal, this distribution would peak at 1 . This distribution peaks at approximately 0.26 ; therefore the daughter's momentum is typically $26 \%$ of the tau lepton's momentum.

\section{A.3 Estimating the Energy of the Tau Lepton}

The tau lepton's energy was difficult to measure directly. There was no estimate from the spectrometer because the tau lepton never left the emulsion. Multiple scattering measurements of the tau lepton's momentum were unreliable due to the short length of the tau lepton track.

The momenta of the daughters in the tau lepton decay were measured; these were used to estimate the tau lepton's momentum. Tau lepton decays were simulated in the Monte Carlo, and the daughters's momenta and the tau lepton's momentum were compared to establish a relationship between the two; this relationship was used to estimate the tau momentum of the tau lepton, Fig. A.2 shows the distribution comparing these momenta for the kink events. The X-axis is the daughter's momentum divided by the tau lepton's momentum. The Y-axis is the normalized weight of the events. If the daughter's momentum and the tau lepton's momentum were the equal, this distribution would peak at 1. This distribution peaks approximately at 0.26 ; therefore, the daughter's momentum is typically $26 \%$ of the tau lepton momentum. Fig. A.3 shows the same distribution for 


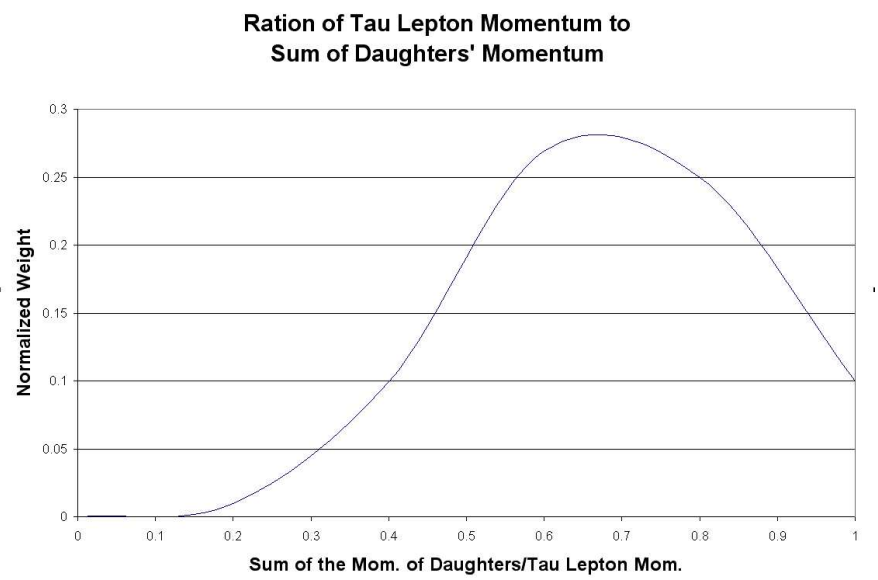

Figure A.3: A comparison of the sum of the three daughters's momenta and the tau lepton's momentum using Monte Carlo data for the trident events. The X-axis is the sum of daughters' momenta divided by the tau lepton's momentum. The Y-axis is the normalized weights of the events. If the sum of the daughters's momenta and the tau lepton's momentum were equal, this distribution would peak at 1 . This distribution peaks at approximately 0.69 ; therefore the sum of daughters's momentum is typically around $69 \%$ of the tau lepton's momentum.

the trident events. The $\mathrm{X}$-axis is the sum of the three daughters's momenta divided by the tau lepton's momentum. The trident distribution peaks at approximately 0.69 . The peaks in these distributions were used to estimate the tau lepton's momentum from the daughters's momenta.

Since the daughters's momenta were measured using multiple scattering, each had an associated uncertainty. The errors on the tau lepton's momenta in the next section originated from these uncertainties. 


\begin{tabular}{cccc} 
Event & $\theta(\mathrm{mrad})$ & $\beta(\mathrm{mrad})$ & $p_{\tau}(\mathrm{GeV})$ \\
\hline 3024_30175 & 28 & 274 & $11.2_{-3.1}^{+5.8}$ \\
3039_01910 & 67 & 88 & $17.8_{-3.4}^{+5.8}$ \\
$3333 \_17665$ & 16 & 206 & $82.6_{-24.7}^{+55.6}$ \\
3334_19920 & 41 & 104 & $46.2_{-10.9}^{+32.3}$ \\
3296_18816 & 140 & 209 & $6.9_{-1.2}^{+4.2}$ \\
$3140 \_22143$ & 41 & 48 & $27.0_{-7.5}^{+14.0}$ \\
\hline
\end{tabular}

Table A.1: Parameters used in the calculation of $Q^{2}$ and $W^{2}$.

\section{A.4 Data}

Table A.1 summarizes $\theta$, the angle between the neutrino and the tau lepton, $\beta$, the angle between the neutrino and the $W^{ \pm}$boson, and $p_{\tau}$, the tau lepton's momentum.

\section{A.5 Results}

Table A.2 summarizes $Q^{2}$ and $W^{2}$ for each of the tau neutrino candidates. The errors from the momentum uncertainties were carried throughout the calculation, but are only shown in the $Q^{2}$ and $W^{2}$ results. The last column specifies whether the event is in the deep inelastic scattering region according to the requirements stated above and using $M^{2}=1$.

\section{A.6 Conclusion}

All but one event are within the deep inelastic scattering region; this event is removed from the set of interactions used to measure the tau neutrino charged-current interaction cross section in the deep inelastic scattering region. 


\begin{tabular}{cccccc} 
Event & $E_{\tau}(\mathrm{GeV})$ & $E_{\nu_{\tau}}$ & $Q^{2}\left(\mathrm{GeV}^{2}\right)$ & $W^{2}\left(\mathrm{GeV}^{2}\right)$ & DIS Region \\
\hline 3024_30175 & 11.34 & 12.31 & $0.39_{-0.1}^{+0.2}$ & $2.55_{-0.7}^{+1.3}$ & $\mathrm{~N}$ \\
$3039 \_01910$ & 17.89 & 31.27 & $4.97_{-1.0}^{+1.6}$ & $22.79_{-4.4}^{+7.4}$ & $\mathrm{Y}$ \\
$3333 \_17765$ & 82.62 & 88.91 & $2.28_{-0.7}^{+1.5}$ & $11.30_{-3.4}^{+7.6}$ & $\mathrm{Y}$ \\
$3334 \_19920$ & 46.23 & 64.30 & $5.69_{-1.34}^{+4.0}$ & $31.45_{-7.4}^{+22.0}$ & $\mathrm{Y}$ \\
$3296 \_18816$ & 7.13 & 11.37 & $3.61_{-0.7}^{+2.5}$ & $5.87_{-1.0}^{+4.0}$ & $\mathrm{Y}$ \\
$3140 \_22143$ & 27.10 & 50.00 & $3.00_{-0.8}^{+1.6}$ & $43.8_{-12.7}^{+25.1}$ & $\mathrm{Y}$ \\
\hline
\end{tabular}

Table A.2: $W^{2}$ and $Q^{2}$ for all tau candidates. 


\section{References}

[1] D. Griffiths, Introduction to Elementary Particles (Wiley, 1987)

[2] F. Reins and C. Cowan, Phys Rev. 92, 830 (1953)

[3] G. Danby et al., Phys. Rev. Lett. 9, 36 (1962)

[4] M. L. Perl et. al., Phys. Rev. Lett. 35, 1489 (1975)

[5] B. R. Martin and G. Shaw, Particle Physics (Wiley, 1999)

[6] K. Kodama et. al., Phys. Lett. B 504, 218-224 (2001)

[7] S. Hatakeyama et al., Phys. Rev. Lett. 81, 2016 (1998)

[8] M. C. Gonzalez-Garcia, C. Pena-Garay, and A. Y. Smirnov, Phys. Rev. D 63, 113004 (2001)

[9] H. Pessard [OPERA Collaboration], arXiv:hep-ex/0504033 (2005)

[10] R. Davis and D. S. Harmer, Bull. Am. Phys. Soc. 4, 217 (1952)

[11] Dark Matter in the Universe (Jerusalem Winter School for Theoretical Physics, 19867), J.N. Bahcall, T. Piran, and S. Weinberg, editors

[12] C. J. Copi and D. N. Schramm, Phys. Rev. D 55, 3389 (1997)

[13] S. Weinberg, Phys. Rev. Lett. 19, 1264 (1967)

[14] A. Salam, in Elementary Particle Theory, ed. N. Svartholm (Almquist and Forlag, 1968)

[15] B. Roe, Particle Physics in the New Millennium (Springer, 1996)

[16] B. R. Martin and G. Shaw, Particle Physics, (Wiley, 1997)

[17] D. Gross and C. Llewellyn-Smith, Nucl. Phys. B 14, 337 (1969)

[18] R. Devenish and A. Cooper-Sarkar, Deep Inelastic Scattering (Oxford, 2004) 
[19] C. G. Callan and D. Gross, Phys. Rev. Lett. 22, 156 (1969)

[20] S. Kretzer and M. H. Reno, Phys. Rev. D 66, 113007 (2002)

[21] H. L. Lai et al. [CTEQ Coll.], Eur. Phys. J. C 12, 375 (2000)

[22] Mathematica, http://www.wolfram.com/

[23] J. Alder, Nucl. Phys. B 46, 573 (1972)

[24] D. Cline and E. A. Paschos, Phys. Rev. D 8, 984 (1973)

[25] S. Eidelman et. al., Phys. Lett. B 592, 1 (2004)

[26] K. Kodama et. al., NIM A 493 (2002)

[27] K. Kodama et. al., NIM A 516 (2004)

[28] Patrick Berghaus, PhD Thesis, Kansas State University (2001)

[29] Jason Sielaff, PhD Thesis, University of Minnesota (2003)

[30] Reinhard Scwienhorst, PhD Thesis, University of Minnesota (2000)

[31] C. Erickson, PhD Thesis, University of Minnesota (2005, in preparation)

[32] R. Brun, et al., ROOT: An Object-Oriented Data Analysis Framework. URL root.cern.ch/

[33] K. Kodama et al., Phys. Lett. B 284, 461 (1992)

[34] R. Ammar et al., Phys. Rev. Lett. 61, 2185 (1988)

[35] CERN, CERNLIB: http://wwwinfo.cern.ch/asdoc/cernlib.html

[36] H. C. Fesefeldt, Simulation of hadronic showers, physics and applications, Technical report PITHA 85-02 (1985)

[37] G. Ingleman, J. Rathsman, and A. Edin, LEPTO-The Lund Monte Carlo for deep inelastic lepton-nuclear scattering, Comput. Phys. Common. 101, 108 (1997)

[38] R. Brun et. al., GEANT Det. Description and Simulation Tool, CERN Program Library, W5013 (1994)

[39] M. E. Duffy et. al., Phys. Rev. Lett. 57, 1522 (1986)

[40] E. Maher, E872 analysis note from 07 Dec. 2004

[41] N. Saoulidou, PhD Thesis, University of Athens (2003) 
[42] P. Lee, Bayesian Statistics: An Introduction (Arnold Publication, 2004)

[43] E531 collaboration (presented by T. Kondo), Fermilab-Conf-80/92-EXP 7550.531

[44] S. A. Rabinowitz et. al., Phys. Rev. Lett. 70, 134 (1993)

[45] T. Bolton, arXiv:hep-ex/9708014v1 (1997)

[46] B. Baller, E872 internal communication, (2003)

[47] C. Albright and C. Jarlskog Nucl. Phys. B84, 467 (1975)

[48] M. Adamovich et al., Phys. Lett. B 284, 453 (1992)

[49] G. A. Alves et al., Phys. Rev. Lett. 70, 722 (1993)

[50] M. J. Leitch et al., Phys. Rev. Lett., 72 (1994)

[51] M. Adamovich et al., Nucl. Phys. B 495, 3 (1997)

[52] S. Fredriksson et. al., Phys Rep 144, 187 (1987)

[53] J. C. Anjos et al., Phys. Rev. Lett. 62, 513 (1989)

[54] S. Barlag et al., Z. Phys. C49, 555 (1991)

[55] K. Riley, M. Hobson, and S. Bence, Mathematical Methods for Physics and Engineering (Cambridge, 2002)

[56] J. R. Taylor, An Introduction to Error Analysis (University Science Books, 1997) 